\title{
Silver-Catalyzed Three-Component Route to Trifluoromethylated 1,2,3-Triazolines Using Aldehydes, Amines, and Trifluorodiazoethane
}

\author{
Anuj Kumar, ${ }^{\dagger}$ Shakir Ahamad, ${ }^{+, \neq}$Ruchir Kant, ${ }^{\S}$ and Kishor Mohanan ${ }^{\dagger, \neq, *}$ \\ ${ }^{\dagger}$ Medicinal \& Process Chemistry Division and ${ }^{\S}$ Molecular and Structural Biology Division, \\ CSIR-Central Drug Research Institute, BS-10/1, Sector 10, Jankipuram extension, Sitapur \\ Road, P.O. Box 173, Lucknow 226031, India \\ ${ }^{\ddagger}$ Academy of Scientific and Innovative Research, New Delhi 110001, India \\ kishor.mohanan@cdri.res.in
}

\section{SUPPORTING INFORMATION}

\section{Contents}

1 General experimental information

S2

2 General Procedure for the synthesis of Trifluoromethylated triazolines S3

3 General Procedure for the synthesis of Trifluoromethylated triazoles S3

4 Characterization data for compounds 4a-4ar

S4-S22

$5 \quad$ Characterization data for compounds $\mathbf{5 a - 5 i}$

S22-S25

$6 \quad$ X-ray data of compound $4 \mathrm{~s}$

$\mathrm{S} 26-\mathrm{S} 27$

7 Copies of ${ }^{1} \mathrm{H},{ }^{13} \mathrm{C}$, and ${ }^{19} \mathrm{~F}$ NMR spectra for compounds $4 \mathrm{a}-4 \mathrm{ar}$

S28-S99

8 Copies of ${ }^{1} \mathrm{H},{ }^{13} \mathrm{C}$, and ${ }^{19} \mathrm{~F}$ NMR spectra for compounds $\mathbf{5 a}-\mathbf{5 i}$

S100-S113 


\section{General experimental information}

Unless otherwise specified, all reactions were performed under air atmosphere in oven dried round-bottom flasks. The reactions were monitored by TLC visualized by UV ( $254 \mathrm{~nm}$ ) and/or with iodine. Column chromatography was performed on 100-200 mesh silica gel using the gradient system ethyl acetate-hexane. NMR data were recorded at Bruker AV $400 \mathrm{MHz}$ in $\mathrm{CDCl}_{3}$ using as internal standards the residual $\mathrm{CHCl}_{3}$ signal for ${ }^{1} \mathrm{H} \mathrm{NMR}(\delta=7.26 \mathrm{ppm})$ and the deuterated solvent signal for ${ }^{13} \mathrm{C}$ NMR $(\delta=77.16 \mathrm{ppm})$. Coupling constants are given in Hertz $(\mathrm{Hz})$ and the classical abbreviations are used to describe the signal multiplicities. Melting points were measured with a Büchi B-540 apparatus and are uncorrected. High resolution mass spectra were obtained using Q-TOF mass spectrometer. All commercially available reagents were used as received. Trifluorodiazoethane was prepared by following a literature procedure. $^{1}$

1. Molander, G. A.; Ryu, D. Angew. Chem., Int. Ed. 2014, 53, 14181. 


\section{Procedures}

\section{General procedure for the synthesis of trifluoromethylated triazolines 4}

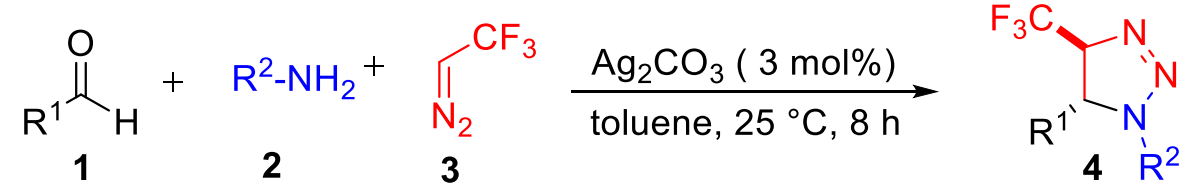

To an oven-dried round bottom flask was added aldehyde $1(0.50 \mathrm{mmol})$, amine $2(0.6 \mathrm{mmol})$, trifluorodiazoethane 3 stock solution in toluene $(1.83 \mathrm{~mL}, 1.50 \mathrm{mmol})$ and $\mathrm{Ag}_{2} \mathrm{CO}_{3}(0.015$ $\mathrm{mmol})$. The reaction mixture was stirred at $25^{\circ} \mathrm{C}$ for $8 \mathrm{~h}$. After the completion of reaction, as indicated by TLC, the reaction mixture was extracted using ethyl acetate $(3 \times 10 \mathrm{~mL})$ and water, and was washed with saturated brine. Finally, the organic layer was dried over anhydrous sodium sulfate and evaporated under reduced pressure. The residue was purified using column chromatography (100-200 mesh silica gel) using ethylacetate/hexane as the eluent to afford the product 4 . The diastereomeric ratio of crude reaction mixture is determined by ${ }^{1} \mathrm{H}$ NMR analysis.

\section{Representative example for the synthesis of compound 4a using $1 \mathrm{mmol}$ of compound $1 \mathrm{a}$}

To an oven-dried round bottom flask was added benzaldehyde 1a (106 mg, $1.0 \mathrm{mmol}$ ), $n$ butylamine $2 \mathrm{a}$ ( $88 \mathrm{mg}, 1.2 \mathrm{mmol}$ ), trifluorodiazoethane 3 stock solution in toluene $(3.66 \mathrm{~mL}$, $3.0 \mathrm{mmol}$ ) and $\mathrm{Ag}_{2} \mathrm{CO}_{3}(8 \mathrm{mg}, 0.03 \mathrm{mmol})$. The reaction mixture was stirred at $25^{\circ} \mathrm{C}$ for $8 \mathrm{~h}$. After the completion of reaction, as indicated by TLC, the reaction mixture was extracted using ethyl acetate $(3 \times 10 \mathrm{~mL})$ and water, and was washed with saturated brine. Finally, the organic layer was dried over anhydrous sodium sulfate and evaporated under reduced pressure. The residue was purified using column chromatography (100-200 mesh silica gel) using ethylacetate/hexane as the eluent to afford the product $4 \mathrm{a}$ ( $258 \mathrm{mg}, 95 \%$ ).

\section{General procedure for the synthesis of trifluoromethylated triazoles $\mathbf{5}$}<smiles>[R]C1C(C(F)(F)F)N=NN1[R]</smiles>

4

$\mathrm{R}^{2}$

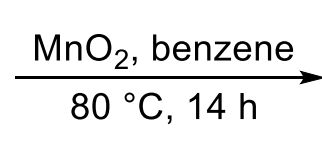<smiles>[R]c1c(C(F)(F)F)nnn1[R]</smiles>

5

To an oven-dried round bottom flask, trifluoromethylated triazoline $4(0.30 \mathrm{mmol})$, activated $\mathrm{MnO}_{2}(1.50 \mathrm{mmol})$ and benzene $(5.0 \mathrm{~mL})$ were added. The resulting mixture was stirred at 80 ${ }^{\circ} \mathrm{C}$ for $14 \mathrm{~h}$. After the complete conversion of triazoline to triazole, as indicated by TLC, the reaction mixture was filtered through celite, and the solvent was evaporated under reduced pressure. The residue was purified using column chromatography (100-200 mesh silica gel) using ethyl acetate /hexane as the eluent to afford triazole $\mathbf{5 .}$ 


\section{Compound 4a: 1-butyl-5-phenyl-4-(trifluoromethyl)-4,5-dihydro-1H-1,2,3-triazole}

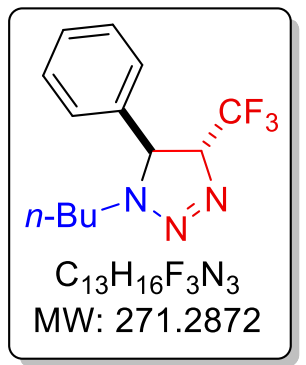

Following the general procedure, treatment of benzaldehyde 1a $(53 \mathrm{mg}$, $0.50 \mathrm{mmol}), \quad n$-butylamine $2 \mathrm{a}(44 \mathrm{mg}, 0.60 \mathrm{mmol})$ with trifluorodiazoethane stock solution 3 in toluene $(1.83 \mathrm{~mL}, 1.50 \mathrm{mmol})$ in the presence of $\mathrm{Ag}_{2} \mathrm{CO}_{3}\left(4 \mathrm{mg}, 0.015 \mathrm{mmol}\right.$ ) at $25^{\circ} \mathrm{C}$ for $8 \mathrm{~h}$ followed by column chromatography afforded the product $4 a(129 \mathrm{mg}, 95 \%)$. Major isomer: colorless liquid, $\mathbf{R}_{f}$ (Ethyl acetate/Hexane: $2 / 98$ ) $=0.25 .{ }^{13} \mathbf{C}$ NMR $\left(100 \mathrm{MHz}, \delta \mathrm{ppm} / \mathrm{CDCl}_{3}\right): 137.7(\mathrm{C}), 129.4(\mathrm{CH}), 129.4(\mathrm{CH}), 129.0(\mathrm{CH})$, $127.1(\mathrm{CH}), 127.0(\mathrm{CH}), 123.8\left(\mathrm{q}, J_{C-F}=221.3 \mathrm{~Hz}, \mathrm{C}\right), 85.1\left(\mathrm{q}, J_{C-F}=22.5 \mathrm{~Hz}, \mathrm{CH}\right), 61.7(\mathrm{~d}, J=1.5$ $\mathrm{Hz}, \mathrm{CH}), 47.8\left(\mathrm{CH}_{2}\right), 29.7\left(\mathrm{CH}_{2}\right), 19.8\left(\mathrm{CH}_{2}\right), 13.5\left(\mathrm{CH}_{3}\right) .{ }^{1} \mathrm{H} \mathrm{NMR}\left(400 \mathrm{MHz}, \delta \mathrm{ppm} / \mathrm{CDCl}_{3}\right): 7.42-$ $7.34(\mathrm{~m}, 3 \mathrm{H}), 7.21-7.19(\mathrm{~m}, 2 \mathrm{H}), 4.75-4.67(\mathrm{~m}, 1 \mathrm{H}), 4.51(\mathrm{~d}, J=10.4 \mathrm{~Hz}, 1 \mathrm{H}), 3.72-3.67(\mathrm{~m}, 1 \mathrm{H})$, 3.36-3.29 (m, 1H), 1.62-1.55 (m, 2H), 1.38-1.25 (m, 2H), $0.88(\mathrm{t}, J=7.2 \mathrm{~Hz}, 3 \mathrm{H}) .{ }^{19} \mathrm{~F}$ NMR (376 $\left.\mathrm{MHz}, \delta \mathrm{ppm} / \mathrm{CDCl}_{3}\right):-73.4$ (s). HRMS for $\mathrm{C}_{13} \mathrm{H}_{17} \mathrm{~F}_{3} \mathrm{~N}_{3}{ }^{+}$: calcd. $[\mathrm{M}+\mathrm{H}]^{+}:$272.1369, found: 272.1357.

\section{Compound 4b: 1-butyl-4-(trifluoromethyl)-4,5-dihydro-1H-1,2,3-triazol-5-yl)phenol}

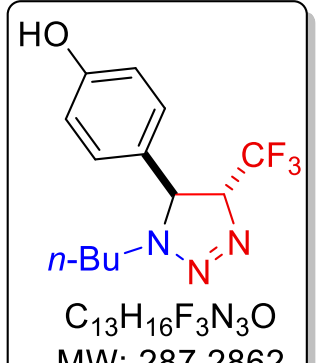

MW: 2872862
Following the general procedure, treatment of 4-hydroxybenzaldehyde 1b $(61 \mathrm{mg}, 0.50 \mathrm{mmol}$ ), $n$-butylamine 2a (44 mg, $0.60 \mathrm{mmol}$ ) with trifluorodiazoethane stock solution 3 in toluene $(1.83 \mathrm{~mL}, 1.50 \mathrm{mmol})$ in the presence of $\mathrm{Ag}_{2} \mathrm{CO}_{3}$ ( $4 \mathrm{mg}, 0.015 \mathrm{mmol}$ ) at $25{ }^{\circ} \mathrm{C}$ for $8 \mathrm{~h}$ followed by column chromatography afforded the product $\mathbf{4 b}$ (103 $\mathrm{mg}, 72 \%)$. Major isomer: white solid, $\mathbf{R}_{\boldsymbol{f}}$ (Ethyl acetate/Hexane: $\left.15 / 85\right)=0.25$. Mp 117-119 ${ }^{\circ} \mathrm{C} .{ }^{13} \mathrm{C}$ NMR $\left(100 \mathrm{MHz}, \delta \mathrm{ppm} / \mathrm{CDCl}_{3}\right): 157.0(\mathrm{C}), 128.9(\mathrm{C}), 128.4(\mathrm{CH})$, $128.4(\mathrm{CH}), 123.8\left(\mathrm{q}, J_{C-F}=221.3 \mathrm{~Hz}, \mathrm{C}\right), 116.5(\mathrm{CH}), 116.5(\mathrm{CH}), 84.1\left(\mathrm{q}, J_{C-F}=22.3 \mathrm{~Hz}, \mathrm{CH}\right), 61.5$ (d, J = $1.4 \mathrm{~Hz}, \mathrm{CH}), 47.6\left(\mathrm{CH}_{2}\right), 29.8\left(\mathrm{CH}_{2}\right), 19.9\left(\mathrm{CH}_{2}\right), 13.6\left(\mathrm{CH}_{3}\right) .{ }^{1} \mathrm{H}$ NMR $(400 \mathrm{MHz}, \delta$ $\left.\mathrm{ppm} / \mathrm{CDCl}_{3}\right): 7.04(\mathrm{~d}, J=8.8 \mathrm{~Hz}, 2 \mathrm{H}), 6.88(\mathrm{dd}, J=8.4 \mathrm{~Hz}, 2.4 \mathrm{~Hz}, 2 \mathrm{H}), 4.74-4.66(\mathrm{~m}, 1 \mathrm{H}), 4.53-$ $4.49(\mathrm{~m}, 1 \mathrm{H}), 3.74-3.67(\mathrm{~m}, 1 \mathrm{H}), 3.35-3.29(\mathrm{~m}, 1 \mathrm{H}), 1.62-1.54(\mathrm{~m}, 2 \mathrm{H}), 1.38-1.27(\mathrm{~m}, 2 \mathrm{H}), 0.89$ $(\mathrm{t}, J=7.2 \mathrm{~Hz}, 3 \mathrm{H}) .{ }^{19} \mathrm{~F} \mathrm{NMR}\left(376 \mathrm{MHz}, \delta \mathrm{ppm} / \mathrm{CDCl}_{3}\right.$ ): -73.7 (s). HRMS for $\mathrm{C}_{13} \mathrm{H}_{17} \mathrm{~F}_{3} \mathrm{~N}_{3} \mathrm{O}^{+}$: calcd. $[\mathrm{M}+\mathrm{H}]^{+}: 288.1318$, found: 288.1317 .

\section{Compound 4c: 1-butyl-5-(4-methoxyphenyl)-4-(trifluoromethyl)-4,5-dihydro-1H-1,2,3- triazole}

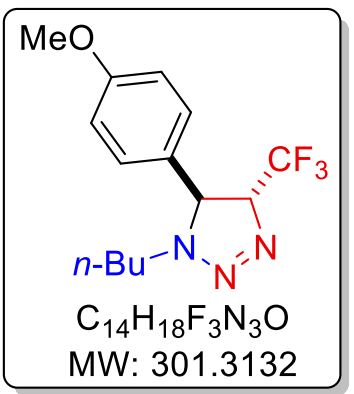

Following the general procedure, treatment of 4methoxybenzaldehyde 1c (68 mg, $0.50 \mathrm{mmol}$ ), $n$-butylamine 2a (44 $\mathrm{mg}, 0.60 \mathrm{mmol}$ ) with trifluorodiazoethane stock solution 3 in toluene (1.83 mL, $1.50 \mathrm{mmol}$ ) in the presence of $\mathrm{Ag}_{2} \mathrm{CO}_{3}(4 \mathrm{mg}, 0.015 \mathrm{mmol})$ at $25{ }^{\circ} \mathrm{C}$ for $8 \mathrm{~h}$ followed by column chromatography afforded the product 4c (136 mg, 90\%). Major isomer: colorless liquid, $\mathbf{R}_{f}$ (Ethyl acetate/Hexane: 5/95) $=0.25 .{ }^{13} \mathrm{C}$ NMR $\left(100 \mathrm{MHz}, \delta \mathrm{ppm} / \mathrm{CDCl}_{3}\right)$ : $160.2(\mathrm{C}), 129.5(\mathrm{C}), 128.3(\mathrm{CH}), 128.3(\mathrm{CH}), 123.9$ (q, $\left.J_{C-F}=276.8 \mathrm{~Hz}, \mathrm{C}\right), 114.9(\mathrm{CH}), 114.9(\mathrm{CH})$, 
$84.8\left(\mathrm{q}, J_{C-F}=27.9 \mathrm{~Hz}, \mathrm{CH}\right), 61.3(\mathrm{~d}, J=2.0 \mathrm{~Hz}, \mathrm{CH}), 55.5\left(\mathrm{CH}_{3}\right), 47.6\left(\mathrm{CH}_{2}\right), 29.8\left(\mathrm{CH}_{2}\right), 19.9\left(\mathrm{CH}_{2}\right)$, $13.6\left(\mathrm{CH}_{3}\right) .{ }^{1} \mathrm{H}$ NMR $\left(400 \mathrm{MHz}, \delta \mathrm{ppm} / \mathrm{CDCl}_{3}\right): 7.13-7.10(\mathrm{~m}, 2 \mathrm{H}), 6.93-6.89(\mathrm{~m}, 2 \mathrm{H}), 4.72-4.64$ $(\mathrm{m}, 1 \mathrm{H}), 4.47(\mathrm{~d}, J=10.4 \mathrm{~Hz}, 1 \mathrm{H}), 3.81(\mathrm{~s}, 3 \mathrm{H}), 3.72-3.65(\mathrm{~m}, 1 \mathrm{H}), 3.33-3.27(\mathrm{~m}, 1 \mathrm{H}), 1.61-1.54$ $(\mathrm{m}, 2 \mathrm{H}), 1.36-1.26(\mathrm{~m}, 2 \mathrm{H}), 0.88(\mathrm{t}, J=7.2 \mathrm{~Hz}, 3 \mathrm{H}) .{ }^{19} \mathrm{~F} \mathrm{NMR}\left(376 \mathrm{MHz}, \delta \mathrm{ppm} / \mathrm{CDCl}_{3}\right):-73.5(\mathrm{~s})$. HRMS for $\mathrm{C}_{14} \mathrm{H}_{19} \mathrm{~F}_{3} \mathrm{~N}_{3} \mathrm{O}^{+}$: calcd. [M+H] $]^{+}: 302.1475$, found: 302.1470 .

Compound 4d:

yl)phenyl)boronic acid

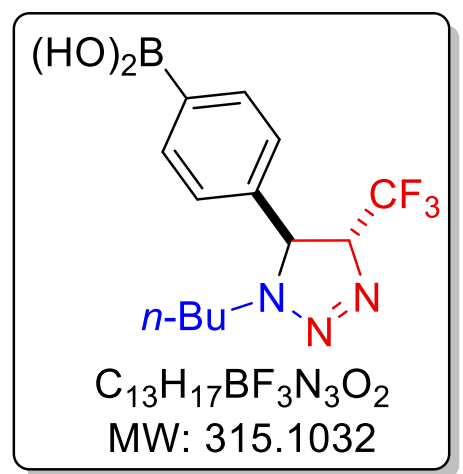

Following the general procedure, treatment of (4formylphenyl)boronic acid $1 \mathbf{d}$ (75 $\mathrm{mg}, 0.50 \mathrm{mmol}), n$-butylamine $2 a(44 \mathrm{mg}, 0.60 \mathrm{mmol}$ ) with trifluorodiazoethane stock solution 3 in toluene $(1.83 \mathrm{~mL}, 1.50 \mathrm{mmol})$ in the presence of $\mathrm{Ag}_{2} \mathrm{CO}_{3}$ (4 $\mathrm{mg}, 0.015 \mathrm{mmol}$ ) at $25{ }^{\circ} \mathrm{C}$ for $8 \mathrm{~h}$ followed by column chromatography afforded the product $\mathbf{4 d}$ ( $87 \mathrm{mg}, 55 \%)$. Major isomer: colorless liquid, $\mathbf{R}_{f}$ (Ethyl acetate/Hexane: $\left.15 / 85\right)=0.30$. ${ }^{13} \mathrm{C}$ NMR (100 MHz, $\left.\delta \mathrm{ppm} / \mathrm{CDCl}_{3}\right): 157.0$ (C), 128.9 (C), 128.4 $(\mathrm{CH}), 128.4(\mathrm{CH}), 123.8\left(\mathrm{q}, J_{\mathrm{C}-F}=277.1 \mathrm{~Hz}, \mathrm{C}\right), 116.5(\mathrm{CH}), 116.5$ $(\mathrm{CH}), 84.1\left(\mathrm{q}, J_{\mathrm{C}-\mathrm{F}}=28.1 \mathrm{~Hz}, \mathrm{CH}\right), 61.5(\mathrm{CH}), 47.6\left(\mathrm{CH}_{2}\right), 29.8\left(\mathrm{CH}_{2}\right), 19.9\left(\mathrm{CH}_{2}\right), 13.7\left(\mathrm{CH}_{3}\right) .{ }^{1} \mathrm{H}$ NMR $\left(400 \mathrm{MHz}, \delta \mathrm{ppm} / \mathrm{CDCl}_{3}\right):$ 7.06-7.02 (m, 2H), 6.90-6.87 (m, 2H), 4.74-4.66 (m, $\left.1 \mathrm{H}\right), 4.51$ $(\mathrm{d}, J=10.0 \mathrm{~Hz}, 1 \mathrm{H}), 3.74-3.68(\mathrm{~m}, 1 \mathrm{H}), 3.35-3.29(\mathrm{~m}, 1 \mathrm{H}), 1.62-1.54(\mathrm{~m}, 2 \mathrm{H}), 1.36-1.25(\mathrm{~m}, 2 \mathrm{H})$, $0.89(\mathrm{t}, J=7.2 \mathrm{~Hz}, 3 \mathrm{H}) .{ }^{19} \mathrm{~F}$ NMR (376 MHz, $\left.\delta \mathrm{ppm} / \mathrm{CDCl}_{3}\right):-73.7$ (s). HRMS for $\mathrm{C}_{13} \mathrm{H}_{18} \mathrm{BF}_{3} \mathrm{~N}_{3} \mathrm{O}_{2}{ }^{+}$: calcd. [M+H-N $]^{+}:$288.1299, found: 288.1316.

Compound 4e: 1-butyl-5-(4-(tert-butyl)phenyl)-4-(trifluoromethyl)-4,5-dihydro-1H-1,2,3triazole

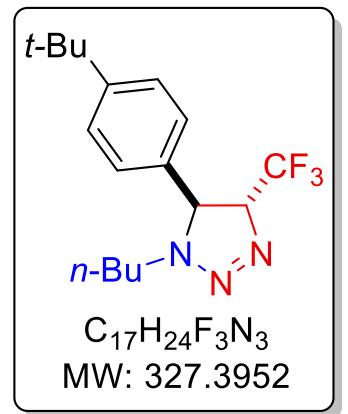

Following the general procedure, treatment of 4-(tertbutyl)benzaldehyde 1 e $(81 \mathrm{mg}, 0.50 \mathrm{mmol}), n$-butylamine $2 \mathrm{a}(44 \mathrm{mg}$, $0.60 \mathrm{mmol}$ ) with trifluorodiazoethane stock solution 3 in toluene (1.83 $\mathrm{mL}, 1.50 \mathrm{mmol}$ ) in the presence of $\mathrm{Ag}_{2} \mathrm{CO}_{3}\left(4 \mathrm{mg}, 0.015 \mathrm{mmol}\right.$ ) at $25^{\circ} \mathrm{C}$ for $10 \mathrm{~h}$ followed by column chromatography afforded the product $4 \mathrm{e}$ (141 mg, 86\%). Major isomer: colorless liquid, $\mathbf{R}_{\boldsymbol{f}}$ (Ethyl acetate/Hexane: $2 / 98)=0.25 .{ }^{13} \mathrm{C}$ NMR $\left(100 \mathrm{MHz}, \delta \mathrm{ppm} / \mathrm{CDCl}_{3}\right): 152.2$ (C), 134.6 (C), $126.7(\mathrm{CH}), 126.7(\mathrm{CH}), 126.4(\mathrm{CH}), 126.4(\mathrm{CH}), 123.9\left(\mathrm{q}, J_{\mathrm{C}-\mathrm{F}}=276.9 \mathrm{~Hz}\right.$, C), $84.9\left(\mathrm{q}, J_{\mathrm{C}-\mathrm{F}}=28.0 \mathrm{~Hz}, \mathrm{CH}\right), 61.3(\mathrm{CH}), 47.7\left(\mathrm{CH}_{2}\right), 34.8(\mathrm{CH}), 31.4\left(\mathrm{CH}_{3}\right), 31.4\left(\mathrm{CH}_{3}\right), 31.4$ $\left(\mathrm{CH}_{3}\right), 29.8\left(\mathrm{CH}_{2}\right), 19.9\left(\mathrm{CH}_{2}\right), 13.7\left(\mathrm{CH}_{3}\right) .{ }^{1} \mathrm{H}$ NMR $\left(400 \mathrm{MHz}, \delta \mathrm{ppm} / \mathrm{CDCl}_{3}\right): 7.42-7.39(\mathrm{~m}, 2 \mathrm{H})$, 7.13-7.10 (m, 2H), 4.75-4.67 (m, 1H), $4.50(\mathrm{~d}, J=10.4 \mathrm{~Hz}, 1 \mathrm{H}), 3.75-3.69(\mathrm{~m}, 1 \mathrm{H}), 3.34-3.28(\mathrm{~m}$, $1 \mathrm{H}), 1.63-1.55(\mathrm{~m}, 2 \mathrm{H}), 1.38-1.30(\mathrm{~m}, 11 \mathrm{H}), 0.89(\mathrm{t}, J=7.6 \mathrm{~Hz}, 3 \mathrm{H}) .{ }^{19} \mathrm{~F}$ NMR $(376 \mathrm{MHz}, \delta$ ppm/CDCl $)$ : -73.5 (s). HRMS for $\mathrm{C}_{17} \mathrm{H}_{25} \mathrm{~F}_{3} \mathrm{~N}_{3}{ }^{+}$: calcd. [M+H] $]^{+}$: 328.1995, found: 328.1991. 


\section{Compound 4f: 1-butyl-5-(p-tolyl)-4-(trifluoromethyl)-4,5-dihydro-1H-1,2,3-triazole}

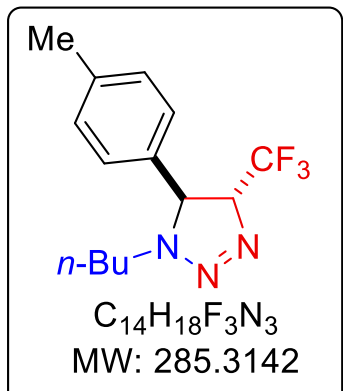

Following the general procedure, treatment of 4-methylbenzaldehyde If $(60 \mathrm{mg}, 0.50 \mathrm{mmol}$ ), $n$-butylamine $2 \mathrm{a}(44 \mathrm{mg}, 0.60 \mathrm{mmol}$ ) with trifluorodiazoethane stock solution 3 in toluene $(1.83 \mathrm{~mL}, 1.50 \mathrm{mmol})$ in the presence of $\mathrm{Ag}_{2} \mathrm{CO}_{3}\left(4 \mathrm{mg}, 0.015 \mathrm{mmol}\right.$ ) at $25^{\circ} \mathrm{C}$ for $8 \mathrm{~h}$ followed by column chromatography afforded the product $\mathbf{4 f}(114 \mathrm{mg}, 80 \%)$. Major isomer: colorless liquid, $\mathbf{R}_{\boldsymbol{f}}$ (Ethyl acetate/Hexane: $\left.2 / 98\right)=0.20$. ${ }^{13} \mathrm{C}$ NMR (100 MHz, $\left.\delta \mathrm{ppm} / \mathrm{CDCl}_{3}\right): 139.1$ (C), 134.7 (C), $130.2(\mathrm{CH})$, $130.2(\mathrm{CH}), 127.0(\mathrm{CH}), 127.0(\mathrm{CH}), 123.9\left(\mathrm{q}, J_{\mathrm{C}-F}=276.9 \mathrm{~Hz}, \mathrm{C}\right), 85.0(\mathrm{q}$, $\left.J_{C-F}=28.2 \mathrm{~Hz}, \mathrm{CH}\right), 61.5(\mathrm{CH}), 47.7\left(\mathrm{CH}_{2}\right), 29.8\left(\mathrm{CH}_{2}\right), 21.2\left(\mathrm{CH}_{3}\right), 19.9\left(\mathrm{CH}_{2}\right), 13.7\left(\mathrm{CH}_{3}\right) .{ }^{1} \mathrm{H}$ NMR $\left(400 \mathrm{MHz}, \delta \mathrm{ppm} / \mathrm{CDCl}_{3}\right): 7.20(\mathrm{~d}, J=8.0 \mathrm{~Hz}, 2 \mathrm{H}), 7.08(\mathrm{~d}, J=8.0 \mathrm{~Hz}, 2 \mathrm{H}), 4.73-4.65(\mathrm{~m}, 1 \mathrm{H})$, $4.48(\mathrm{~d}, J=10.0 \mathrm{~Hz}, 1 \mathrm{H}), 3.72-3.66(\mathrm{~m}, 1 \mathrm{H}), 3.34-3.27(\mathrm{~m}, 1 \mathrm{H}), 2.36(\mathrm{~s}, 3 \mathrm{H}), 1.60-1.54(\mathrm{~m}, 2 \mathrm{H})$, 1.35-1.27 (m, 2H), $0.89(\mathrm{t}, J=7.2 \mathrm{~Hz}, 3 \mathrm{H}) .{ }^{19} \mathrm{~F}$ NMR (376 MHz, $\left.\delta \mathrm{ppm} / \mathrm{CDCl}_{3}\right):-73.4$ (s). HRMS for $\mathrm{C}_{14} \mathrm{H}_{29} \mathrm{~F}_{3} \mathrm{~N}_{3}{ }^{+}$: calcd. $[\mathrm{M}+\mathrm{H}]^{+}:$286.1526, found: 286.1520 . Minor isomer: yellow liquid. $\mathbf{R}_{\boldsymbol{f}}$ (Ethyl acetate/Hexane: 5/95) = 0.30. ${ }^{13} \mathrm{C} \mathrm{NMR}\left(100 \mathrm{MHz}, \delta \mathrm{ppm} / \mathrm{CDCl}_{3}\right): 139.0(\mathrm{C}), 129.4(\mathrm{CH})$, $129.4(\mathrm{CH}), 129.4(\mathrm{CH}), 129.4(\mathrm{CH}), 128.1(\mathrm{C}), 123.2$ (q, $\left.J_{C-F}=222.2 \mathrm{~Hz}, \mathrm{C}\right), 79.8$ (q, J J $J_{C-F}=22.1$ $\mathrm{Hz}, \mathrm{CH}), 62.5(\mathrm{CH}), 47.4\left(\mathrm{CH}_{2}\right), 30.3\left(\mathrm{CH}_{2}\right), 21.3\left(\mathrm{CH}_{3}\right), 20.1\left(\mathrm{CH}_{2}\right), 13.8\left(\mathrm{CH}_{3}\right) .{ }^{1} \mathrm{H}$ NMR $(400 \mathrm{MHz}$, $\left.\delta \mathrm{ppm} / \mathrm{CDCl}_{3}\right): 7.15(\mathrm{~d}, J=8.0 \mathrm{~Hz}, 2 \mathrm{H}), 7.01(\mathrm{~d}, J=8.0 \mathrm{~Hz}, 2 \mathrm{H}), 5.00-4.90(\mathrm{~m}, 1 \mathrm{H}), 4.79(\mathrm{~d}, J=$ $13.2 \mathrm{~Hz}, 1 \mathrm{H}), 3.87-3.80(\mathrm{~m}, 1 \mathrm{H}), 3.19-3.12(\mathrm{~m}, 1 \mathrm{H}), 2.35(\mathrm{~s}, 3 \mathrm{H}), 1.65-1.60(\mathrm{~m}, 2 \mathrm{H}), 1.38-1.30$ $(\mathrm{m}, 2 \mathrm{H}), 0.90(\mathrm{t}, J=7.2 \mathrm{~Hz}, 3 \mathrm{H}) .{ }^{19} \mathrm{~F}$ NMR $\left(376 \mathrm{MHz}, \delta \mathrm{ppm} / \mathrm{CDCl}_{3}\right):-66.2$ (s).

\section{Compound 4g: 5-([1,1'-biphenyl]-4-yl)-1-butyl-4-(trifluoromethyl)-4,5-dihydro-1H-1,2,3- triazole}

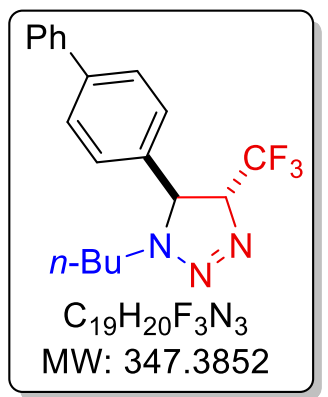

Following the general procedure, treatment of [1,1'-biphenyl]-4carbaldehyde $1 \mathrm{~g}$ (91 $\mathrm{mg}, 0.50 \mathrm{mmol}$ ), $n$-butylamine 2a (44 mg, 0.60 $\mathrm{mmol})$ with trifluorodiazoethane stock solution 3 in toluene $(1.83 \mathrm{~mL}$, $1.50 \mathrm{mmol}$ ) in the presence of $\mathrm{Ag}_{2} \mathrm{CO}_{3}\left(4 \mathrm{mg}, 0.015 \mathrm{mmol}\right.$ ) at $25^{\circ} \mathrm{C}$ for 10 $\mathrm{h}$ followed by column chromatography afforded the product $\mathbf{4 g}(129 \mathrm{mg}$, 74\%). Major isomer: white solid, $\mathbf{R}_{\boldsymbol{f}}$ (Ethyl acetate/Hexane: $\left.2 / 98\right)=0.22$. Mp 40-42 ${ }^{\circ} \mathrm{C} .{ }^{13} \mathrm{C}$ NMR (100 MHz, $\delta$ ppm/CDCl $): 142.2$ (C), 140.2 (C), $136.6(\mathrm{C}), 129.0(\mathrm{CH}), 129.0(\mathrm{CH}), 128.2(\mathrm{CH}), 128.2(\mathrm{CH}), 127.9(\mathrm{CH}), 127.5(\mathrm{CH}), 127.5(\mathrm{CH})$, $127.2(\mathrm{CH}), 127.2(\mathrm{CH}), 123.8\left(\mathrm{q}, J_{C-F}=276.8 \mathrm{~Hz}, \mathrm{C}\right), 84.6\left(\mathrm{q}, J_{C-F}=28.1 \mathrm{~Hz}, \mathrm{CH}\right), 61.5(\mathrm{~d}, J=1.7$ $\mathrm{Hz}, \mathrm{CH}), 47.9\left(\mathrm{CH}_{2}\right), 29.8\left(\mathrm{CH}_{2}\right), 19.9\left(\mathrm{CH}_{2}\right), 13.7\left(\mathrm{CH}_{3}\right) .{ }^{1} \mathrm{H} \mathbf{N M R}\left(400 \mathrm{MHz}, \delta \mathrm{ppm} / \mathrm{CDCl}_{3}\right): 7.52$ $(\mathrm{d}, J=6.4 \mathrm{~Hz}, 2 \mathrm{H}), 7.48(\mathrm{~d}, J=6.0, \mathrm{~Hz}, 2 \mathrm{H}), 7.36(\mathrm{t}, J=11.6 \mathrm{~Hz}, 2 \mathrm{H}), 7.29(\mathrm{~d}, J=6.0 \mathrm{~Hz}, 1 \mathrm{H}), 7.17$ $(\mathrm{d}, J=6.4 \mathrm{~Hz}, 2 \mathrm{H}), 4.69-4.63(\mathrm{~m}, 1 \mathrm{H}), 4.46(\mathrm{~d}, J=8.4 \mathrm{~Hz}, 1 \mathrm{H}), 3.68-3.62(\mathrm{~m}, 1 \mathrm{H}), 3.30-3.24(\mathrm{~m}$, $1 \mathrm{H}), 1.55-1.49(\mathrm{~m}, 2 \mathrm{H}), 1.28-1.19(\mathrm{~m}, 2 \mathrm{H}), 0.81(\mathrm{t}, J=6.0 \mathrm{~Hz}, 3 \mathrm{H}) .{ }^{19} \mathrm{~F}$ NMR $(376 \mathrm{MHz}, \delta$ ppm/ $\mathrm{CDCl}_{3}$ ): -73.3 (s). HRMS for $\mathrm{C}_{19} \mathrm{H}_{21} \mathrm{~F}_{3} \mathrm{~N}_{3}{ }^{+}$: calcd. $[\mathrm{M}+\mathrm{H}]^{+}: 348.1682$, found: 348.1682 . 


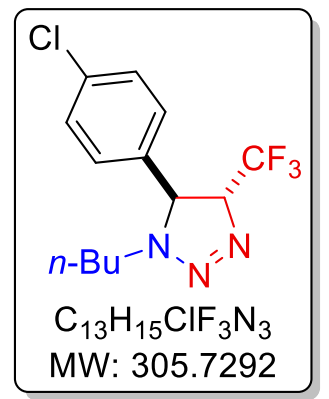

Following the general procedure, treatment of 4-chlorobenzaldehyde $\mathbf{1 h}$ (70 $\mathrm{mg}, 0.50 \mathrm{mmol}$ ), $n$-butylamine $2 \mathrm{a}(44 \mathrm{mg}, 0.60 \mathrm{mmol}$ ) with trifluorodiazoethane stock solution 3 in toluene $(1.83 \mathrm{~mL}, 1.50 \mathrm{mmol})$ in the presence of $\mathrm{Ag}_{2} \mathrm{CO}_{3}$ ( $4 \mathrm{mg}, 0.015 \mathrm{mmol}$ ) at $25^{\circ} \mathrm{C}$ for $8 \mathrm{~h}$ followed by column chromatography afforded the product $4 \mathrm{~h}$ (127 mg, 83\%). Major isomer: colorless liquid, $\mathbf{R}_{\boldsymbol{f}}$ (Ethyl acetate/Hexane: $\left.5 / 95\right)=0.30 .{ }^{13} \mathbf{C} \mathbf{N M R}$ $\left(100 \mathrm{MHz}, \delta \mathrm{ppm} / \mathrm{CDCl}_{3}\right): 136.3(\mathrm{C}), 135.1(\mathrm{C}), 129.8(\mathrm{CH}), 129.8(\mathrm{CH})$, $128.4(\mathrm{CH}), 128.4(\mathrm{CH}), 123.7\left(\mathrm{q}, J_{C-F}=278.4 \mathrm{~Hz}, \mathrm{C}\right), 85.2\left(q, J_{C-F}=28.3 \mathrm{~Hz}, \mathrm{CH}\right), 61.2(\mathrm{CH}), 48.0$ $\left(\mathrm{CH}_{2}\right), 29.7\left(\mathrm{CH}_{2}\right), 19.9\left(\mathrm{CH}_{2}\right), 13.7\left(\mathrm{CH}_{3}\right) .{ }^{1} \mathrm{H}$ NMR $\left(400 \mathrm{MHz}, \delta \mathrm{ppm} / \mathrm{CDCl}_{3}\right): 7.40-7.35(\mathrm{~m}, 2 \mathrm{H})$, 7.17-7.13 (m, 2H), 4.69-4.62 (m, 1H), 4.50-4.45 (m, 1H), 3.71-3.65 (m, 1H), 3.35-3.27 (m, 1H),

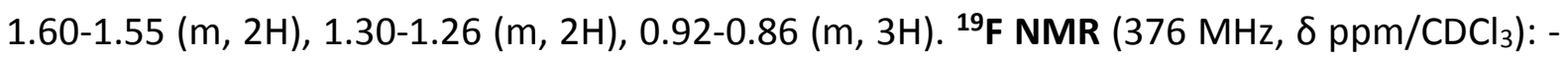
73.3 (s). HRMS for $\mathrm{C}_{13} \mathrm{H}_{16} \mathrm{ClF}_{3} \mathrm{~N}_{3}{ }^{+}$: calcd. [M+H] $]^{+}: 306.0979$, found: 306.0974; calcd. [M+2+H] ${ }^{+}$ for $\mathrm{C}_{13} \mathrm{H}_{16}{ }^{37} \mathrm{ClF}_{3} \mathrm{~N}_{3}{ }^{+}$: 308.0950, found: 308.0945 .

Compound 4i: 1-butyl-5-(4-fluorophenyl)-4-(trifluoromethyl)-4,5-dihydro-1H-1,2,3-triazole

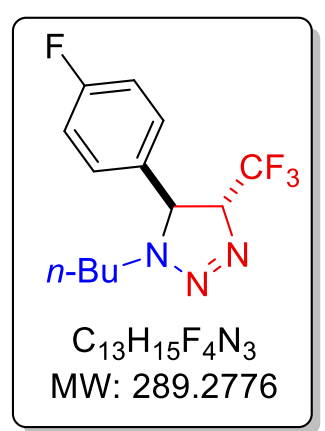

Following the general procedure, treatment of 4-fluorobenzaldehyde $\mathbf{1 i}$ (62.0 $\mathrm{mg}, 0.50 \mathrm{mmol}$ ), $n$-butylamine $2 \mathrm{a}(44 \mathrm{mg}, 0.60 \mathrm{mmol}$ ) with trifluorodiazoethane stock solution 3 in toluene $(1.83 \mathrm{~mL}, 1.50 \mathrm{mmol})$ in the presence of $\mathrm{Ag}_{2} \mathrm{CO}_{3}$ ( $4 \mathrm{mg}, 0.015 \mathrm{mmol}$ ) at $25^{\circ} \mathrm{C}$ for $10 \mathrm{~h}$ followed by column chromatography afforded the product 4i (127 mg, 88\%). Major isomer: colorless liquid, $\mathbf{R}_{f}$ (Ethyl acetate/Hexane: $\left.5 / 95\right)=0.30 .{ }^{13} \mathbf{C}$ NMR $\left(100 \mathrm{MHz}, \delta \mathrm{ppm} / \mathrm{CDCl}_{3}\right): 163.1\left(\mathrm{~d}, J_{\mathrm{C}-F}=247.0 \mathrm{~Hz}, \mathrm{C}\right), 133.5(\mathrm{~d}, J=3.2 \mathrm{~Hz}$, C), $128.8(\mathrm{~d}, J=8.3 \mathrm{~Hz}, \mathrm{CH}), 128.8(\mathrm{~d}, J=8.3 \mathrm{~Hz}, \mathrm{CH}), 123.7\left(\mathrm{q}, J_{C-F}=276.8\right.$ $\mathrm{Hz}, \mathrm{C}), 116.6(\mathrm{~d}, J=21.8 \mathrm{~Hz}, \mathrm{CH}), 116.6(\mathrm{~d}, J=21.8 \mathrm{~Hz}, \mathrm{CH}), 85.2\left(\mathrm{q}, J_{C-F}=28.1 \mathrm{~Hz}, \mathrm{CH}\right), 61.2(\mathrm{~d}$, $J=1.9 \mathrm{~Hz}, \mathrm{CH}), 49.9\left(\mathrm{CH}_{2}\right), 29.7\left(\mathrm{CH}_{2}\right), 19.9\left(\mathrm{CH}_{2}\right), 13.6\left(\mathrm{CH}_{3}\right) .{ }^{1} \mathrm{H} \mathrm{NMR}\left(400 \mathrm{MHz}, \delta \mathrm{ppm} / \mathrm{CDCl}_{3}\right)$ : 7.20-7.16 (m, 2H), 7.11-7.06 (m, 2H), 4.69-4.63 (m, 1H), $4.49(\mathrm{~d}, J=10.4 \mathrm{~Hz}, 1 \mathrm{H}), 3.70-3.65(\mathrm{~m}$, $1 \mathrm{H}), 3.35-3.28(\mathrm{~m}, 1 \mathrm{H}), 1.61-1.54(\mathrm{~m}, 2 \mathrm{H}), 1.36-1.27(\mathrm{~m}, 2 \mathrm{H}), 0.88(\mathrm{t}, J=7.2 \mathrm{~Hz}, 3 \mathrm{H}) .{ }^{19} \mathrm{~F} \mathrm{NMR}$ (376 MHz, $\delta \mathrm{ppm} / \mathrm{CDCl}_{3}$ ): -73.4 (s), -112.4 (s). HRMS for $\mathrm{C}_{13} \mathrm{H}_{16} \mathrm{~F}_{4} \mathrm{~N}_{3}{ }^{+}$: calcd. $[\mathrm{M}+\mathrm{H}]^{+}:$: 290.1275, found: 290.1273 .

\section{Compound 4j: methyl 4-(1-butyl-4-(trifluoromethyl)-4,5-dihydro-1H-1,2,3-triazol-5- yl)benzoate}

Following the general procedure, treatment of methyl 4-formylbenzoate $1 \mathbf{j}$ (82 $\mathrm{mg}, 0.50$ mmol), $n$-butylamine 2 a (44 $\mathrm{mg}, 0.60 \mathrm{mmol}$ ) with trifluorodiazoethane stock solution 3 in toluene $(1.83 \mathrm{~mL}, 1.50 \mathrm{mmol})$ in the presence of $\mathrm{Ag}_{2} \mathrm{CO}_{3}(4 \mathrm{mg}, 0.015 \mathrm{mmol})$ at $25^{\circ} \mathrm{C}$ for $10 \mathrm{~h}$ 
followed by column chromatography afforded the product $4 \mathbf{j}$ (100 mg, 61\%). Major isomer:

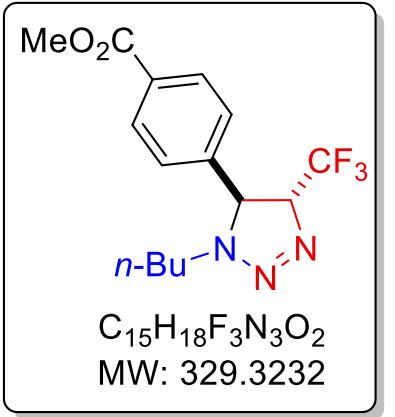
colourless liquid, $\mathbf{R}_{f}$ (Ethyl acetate/Hexane: $5 / 95$ ) $=0.25 .{ }^{13} \mathbf{C}$ NMR (100 MHz, $\delta \mathrm{ppm} / \mathrm{CDCl}_{3}$ ): 166.4 (C), 142.7 (C), 131.1 (C), 130.8 (CH), $130.8(\mathrm{CH}), 127.1(\mathrm{CH}), 127.1(\mathrm{CH}), 123.6\left(\mathrm{q}, J_{C-F}=276.7 \mathrm{~Hz}, \mathrm{C}\right), 85.8$ (q, $\left.J_{C-F}=28.3 \mathrm{~Hz}, \mathrm{CH}\right), 61.6(\mathrm{CH}), 52.5\left(\mathrm{CH}_{3}\right), 48.2\left(\mathrm{CH}_{2}\right), 29.8\left(\mathrm{CH}_{2}\right)$, $19.9\left(\mathrm{CH}_{2}\right), 13.7\left(\mathrm{CH}_{3}\right) .{ }^{1} \mathrm{H}$ NMR $\left(400 \mathrm{MHz}, \delta \mathrm{ppm} / \mathrm{CDCl}_{3}\right): 8.07(\mathrm{~d}, J=$ $6.4 \mathrm{~Hz}, 2 \mathrm{H}), 7.29(\mathrm{~d}, J=6.4 \mathrm{~Hz}, 2 \mathrm{H}), 4.74-4.67(\mathrm{~m}, 1 \mathrm{H}), 4.54(\mathrm{~d}, J=8.0$ $\mathrm{Hz}, 1 \mathrm{H}), 3.93(\mathrm{~s}, 3 \mathrm{H}), 3.70-3.67(\mathrm{~m}, 1 \mathrm{H}), 3.36-3.31(\mathrm{~m}, 1 \mathrm{H}), 1.61-1.55$ $(\mathrm{m}, 2 \mathrm{H}), 1.39-1.27(\mathrm{~m}, 2 \mathrm{H}), 0.88(\mathrm{t}, J=6.0 \mathrm{~Hz}, 3 \mathrm{H}) .{ }^{19} \mathrm{~F} \mathrm{NMR}(376 \mathrm{MHz}$, $\delta \mathrm{ppm} / \mathrm{CDCl}_{3}$ ): -73.3 (s). HRMS for $\mathrm{C}_{15} \mathrm{H}_{19} \mathrm{~F}_{3} \mathrm{~N}_{3} \mathrm{O}_{2}{ }^{+}$: calcd. [M+H] $]^{+}: 330.1424$, found: 330.1416 .

Compound 4k: 1-butyl-5-(4-nitrophenyl)-4-(trifluoromethyl)-4,5-dihydro-1H-1,2,3-triazole

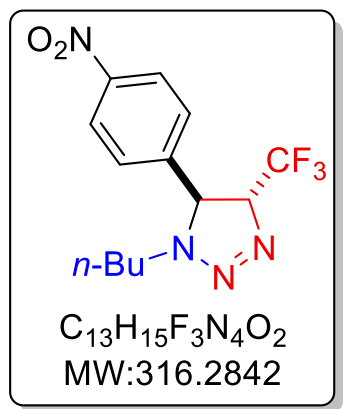

Following the general procedure, treatment of 4-nitrobenzaldehyde $\mathbf{1 k}$ (76 mg, $0.50 \mathrm{mmol}$ ), $n$-butylamine $2 \mathrm{a}$ (44 $\mathrm{mg}, 0.60 \mathrm{mmol}$ ) with trifluorodiazoethane stock solution 3 in toluene $(1.83 \mathrm{~mL}, 1.50 \mathrm{mmol})$ in the presence of $\mathrm{Ag}_{2} \mathrm{CO}_{3}(4 \mathrm{mg}, 0.015 \mathrm{mmol})$ at $25{ }^{\circ} \mathrm{C}$ for $14 \mathrm{~h}$ followed by column chromatography afforded the product $\mathbf{4 k}$ (103 $\mathrm{mg}, 65 \%)$. Major isomer: yellow solid, $\mathbf{R}_{\boldsymbol{f}}$ (Ethyl acetate/Hexane: $\left.5 / 95\right)=0.20$. $\mathbf{M p}$ 50-52 ${ }^{\circ} \mathrm{C} .{ }^{13} \mathrm{C}$ NMR (100 MHz, $\left.\delta \mathrm{ppm} / \mathrm{CDCl}_{3}\right): 148.5$ (C), 144.9 (C), 128.1 (CH), $128.1(\mathrm{CH}), 124.8(\mathrm{CH}), 124.8(\mathrm{CH}), 123.4\left(\mathrm{q}, J_{\mathrm{C}-\mathrm{F}}=276.8 \mathrm{~Hz}, \mathrm{C}\right), 85.5$ $\left(q, J_{C-F}=28.6 \mathrm{~Hz}, \mathrm{CH}\right), 61.3(\mathrm{CH}), 48.5\left(\mathrm{CH}_{2}\right), 29.7\left(\mathrm{CH}_{2}\right), 19.9\left(\mathrm{CH}_{2}\right), 13.6\left(\mathrm{CH}_{3}\right) .{ }^{1} \mathrm{H}$ NMR $(400$ $\left.\mathrm{MHz}, \delta \mathrm{ppm} / \mathrm{CDCl}_{3}\right): 8.27(\mathrm{~d}, J=8.4 \mathrm{~Hz}, 2 \mathrm{H}), 7.43(\mathrm{~d}, J=8.8 \mathrm{~Hz}, 2 \mathrm{H}), 4.74-4.66(\mathrm{~m}, 1 \mathrm{H}), 4.58(\mathrm{~d}$, $J=10.8 \mathrm{~Hz}, 1 \mathrm{H}), 3.72-3.66(\mathrm{~m}, 1 \mathrm{H}), 3.39-3.32(\mathrm{~m}, 1 \mathrm{H}), 1.63-1.56(\mathrm{~m}, 2 \mathrm{H}), 1.39-1.28(\mathrm{~m}, 2 \mathrm{H})$, $0.89(\mathrm{t}, J=7.2 \mathrm{~Hz}, 3 \mathrm{H}) .{ }^{19} \mathrm{~F} \mathrm{NMR}\left(376 \mathrm{MHz}, \delta \mathrm{ppm} / \mathrm{CDCl}_{3}\right):-73.1$ (s). HRMS for $\mathrm{C}_{13} \mathrm{H}_{16} \mathrm{~F}_{3} \mathrm{~N}_{4} \mathrm{O}_{2}{ }^{+}$: calcd. $[\mathrm{M}+\mathrm{H}]^{+}: 317.1220$, found: 317.1220 .

\section{Compound 4I: 1-butyl-5-(3-methoxyphenyl)-4-(trifluoromethyl)-4,5-dihydro-1H-1,2,3- triazole}

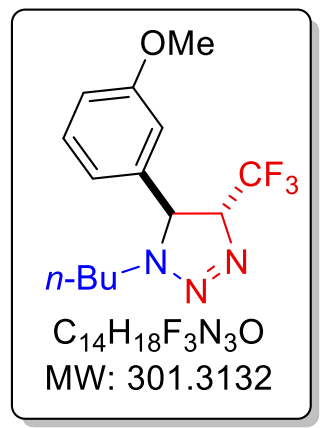

Following the general procedure, treatment of 3-methoxybenzaldehyde 1) (68 mg, $0.50 \mathrm{mmol}$ ), $n$-butylamine $2 \mathrm{a}(44 \mathrm{mg}, 0.60 \mathrm{mmol}$ ) with trifluorodiazoethane stock solution 3 in toluene $(1.83 \mathrm{~mL}, 1.50 \mathrm{mmol})$ in the presence of $\mathrm{Ag}_{2} \mathrm{CO}_{3}\left(4 \mathrm{mg}, 0.015 \mathrm{mmol}\right.$ ) at $25{ }^{\circ} \mathrm{C}$ for $8 \mathrm{~h}$ followed by column chromatography afforded the product 41 (122 mg, 81\%). Major isomer: colorless liquid, $\mathbf{R}_{f}$ (Ethyl acetate/Hexane: $3 / 97$ ) $=0.25 .{ }^{13} \mathbf{C}$ NMR $\left(100 \mathrm{MHz}, \delta \mathrm{ppm} / \mathrm{CDCl}_{3}\right): 160.5(\mathrm{C}), 139.3(\mathrm{C}), 130.6(\mathrm{CH}), 123.8\left(\mathrm{q}, J_{\mathrm{C}-F}=\right.$ $276.8 \mathrm{~Hz}, \mathrm{C}), 119.2(\mathrm{CH}), 114.4(\mathrm{CH}), 112.5(\mathrm{CH}), 85.0$ (q, J J-F $=28.1 \mathrm{~Hz}$, $\mathrm{CH}), 61.6(\mathrm{~d}, \mathrm{~J}=1.9 \mathrm{~Hz}, \mathrm{CH}), 55.5\left(\mathrm{CH}_{3}\right), 47.9\left(\mathrm{CH}_{2}\right), 29.8\left(\mathrm{CH}_{2}\right), 19.9\left(\mathrm{CH}_{2}\right), 13.7\left(\mathrm{CH}_{3}\right) .{ }^{1} \mathbf{H}$ NMR (400 MHz, $\left.\delta \mathrm{ppm} / \mathrm{CDCl}_{3}\right): 7.33-7.28(\mathrm{~m}, 1 \mathrm{H}), 6.90-6.88(\mathrm{~m}, 1 \mathrm{H}), 6.78(\mathrm{~d}, J=7.6 \mathrm{~Hz}, 1 \mathrm{H}), 6.71$ $(\mathrm{t}, J=2.0 \mathrm{~Hz}, 1 \mathrm{H}), 4.76-4.68(\mathrm{~m}, 1 \mathrm{H}), 4.47(\mathrm{dd}, J=10.4 \mathrm{~Hz}, 2.4 \mathrm{~Hz}, 1 \mathrm{H}), 3.81-3.80(\mathrm{~m}, 3 \mathrm{H}), 3.76-$ $3.68(\mathrm{~m}, 1 \mathrm{H}), 3.37-3.30(\mathrm{~m}, 1 \mathrm{H}), 1.63-1.55(\mathrm{~m}, 2 \mathrm{H}), 1.37-1.26(\mathrm{~m}, 2 \mathrm{H}), 0.91-0.87(\mathrm{~m}, 3 \mathrm{H}) .{ }^{19} \mathbf{F}$ 
NMR (376 MHz, $\delta \mathrm{ppm} / \mathrm{CDCl}_{3}$ ): -73.4 (s). HRMS for $\mathrm{C}_{14} \mathrm{H}_{19} \mathrm{~F}_{3} \mathrm{~N}_{3} \mathrm{O}^{+}$: calcd. $[\mathrm{M}+\mathrm{H}]^{+}: 302.1475$, found: 302.1473 .

Compound 4m: 1-butyl-5-(3-fluorophenyl)-4-(trifluoromethyl)-4,5-dihydro-1H-1,2,3triazole

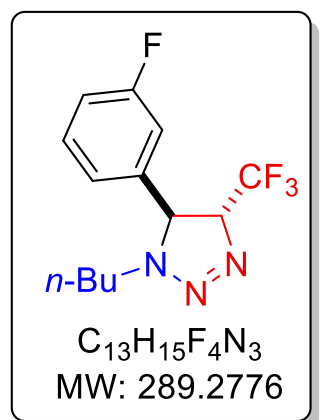

Following the general procedure, treatment of 3-fluorobenzaldehyde $1 \mathrm{~m}$ (62 $\mathrm{mg}, 0.50 \mathrm{mmol}$ ), $n$-butylamine $2 \mathrm{a}(44 \mathrm{mg}, 0.60 \mathrm{mmol}$ ) with trifluorodiazoethane stock solution 3 in toluene $(1.83 \mathrm{~mL}, 1.50 \mathrm{mmol})$ in the presence of $\mathrm{Ag}_{2} \mathrm{CO}_{3}\left(4 \mathrm{mg}, 0.015 \mathrm{mmol}\right.$ ) at $25{ }^{\circ} \mathrm{C}$ for $8 \mathrm{~h}$ followed by column chromatography afforded the product $4 \mathrm{~m}(124 \mathrm{mg}, 86 \%)$. Major isomer: colorless liquid, $\mathbf{R}_{\boldsymbol{f}}$ (Ethyl acetate/Hexane: $\left.5 / 95\right)=0.30 .{ }^{13} \mathrm{C}$ NMR $\left(100 \mathrm{MHz}, \delta \mathrm{ppm} / \mathrm{CDCl}_{3}\right): 163.3\left(\mathrm{~d}, J_{C-F}=246.9 \mathrm{~Hz}, \mathrm{C}\right), 140.3(\mathrm{~d}, J=6.8 \mathrm{~Hz}$, C), $131.2(\mathrm{~d}, J=8.2 \mathrm{~Hz}, \mathrm{CH}), 123.7\left(\mathrm{q}, J_{C-F}=276.8 \mathrm{~Hz}, \mathrm{C}\right), 122.5(\mathrm{~d}, J=2.9$ $\mathrm{Hz}, \mathrm{CH}$ ), 116.1 (d, J = 21.0 Hz, CH), $114.0(\mathrm{~d}, J=22.3 \mathrm{~Hz}, \mathrm{CH}), 85.2\left(\mathrm{q}, J_{C-F}=28.3 \mathrm{~Hz}, \mathrm{CH}\right), 61.3$ $(\mathrm{CH}), 48.0\left(\mathrm{CH}_{2}\right), 29.7\left(\mathrm{CH}_{2}\right), 19.8\left(\mathrm{CH}_{2}\right), 13.5\left(\mathrm{CH}_{3}\right) .{ }^{1} \mathrm{H}$ NMR $\left(400 \mathrm{MHz}, \delta \mathrm{ppm} / \mathrm{CDCl}_{3}\right):$ 7.39$7.35(\mathrm{~m}, 1 \mathrm{H}), 7.05(\mathrm{td}, J=4.8 \mathrm{~Hz}, 1.6 \mathrm{~Hz}, 1 \mathrm{H}), 7.00(\mathrm{~d}, J=6.6 \mathrm{~Hz}, 1 \mathrm{H}), 6.91(\mathrm{~d}, J=7.6 \mathrm{~Hz}, 1 \mathrm{H})$, 4.72-4.65 (m, 1H), $4.49(\mathrm{~d}, J=8.4 \mathrm{~Hz}, 1 \mathrm{H}), 3.73-3.67(\mathrm{~m}, 1 \mathrm{H}), 3.36-3.31(\mathrm{~m}, 1 \mathrm{H}), 1.61-1.55(\mathrm{~m}$, $2 \mathrm{H}), 1.36-1.26(\mathrm{~m}, 2 \mathrm{H}), 0.88(\mathrm{t}, J=6.0 \mathrm{~Hz}, 3 \mathrm{H}) .{ }^{19} \mathrm{~F} \mathrm{NMR}\left(376 \mathrm{MHz}, \delta \mathrm{ppm} / \mathrm{CDCl}_{3}\right):-73.4(\mathrm{~s}),-$ 111.0 (s). HRMS for $\mathrm{C}_{13} \mathrm{H}_{16} \mathrm{~F}_{4} \mathrm{~N}_{3}{ }^{+}$: calcd. [M+H] $]^{+}: 290.1275$, found: 290.1268 .

\section{Compound 4n: 1-butyl-5-(o-tolyl)-4-(trifluoromethyl)-4,5-dihydro-1H-1,2,3-triazole}

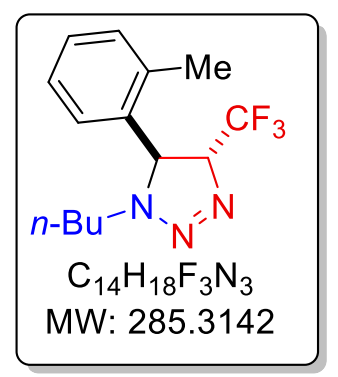

Following the general procedure, treatment of 2-methylbenzaldehyde 1n (60 $\mathrm{mg}, 0.50 \mathrm{mmol})$, $n$-butylamine $2 \mathrm{a}(44 \mathrm{mg}, 0.60 \mathrm{mmol})$ with trifluorodiazoethane stock solution 3 in toluene $(1.83 \mathrm{~mL}, 1.50 \mathrm{mmol})$ in the presence of $\mathrm{Ag}_{2} \mathrm{CO}_{3}$ ( $4 \mathrm{mg}, 0.015 \mathrm{mmol}$ ) at $25^{\circ} \mathrm{C}$ for $10 \mathrm{~h}$ followed by column chromatography afforded the product $4 \mathrm{n}$ (114 $\mathrm{mg}, 80 \%)$. Major isomer: colorless liquid, $\mathbf{R}_{\boldsymbol{f}}$ (Ethyl acetate/Hexane: $\left.2 / 98\right)=0.22 .{ }^{13} \mathrm{C}$ NMR $\left(100 \mathrm{MHz}, \delta \mathrm{ppm} / \mathrm{CDCl}_{3}\right): 135.9(\mathrm{CH}), 135.6(\mathrm{C}), 131.5(\mathrm{C}), 128.8(\mathrm{CH})$, $128.8(\mathrm{CH}), 127.3(\mathrm{CH}), 124.0\left(\mathrm{q}, J_{C-F}=221.5 \mathrm{~Hz}, \mathrm{C}\right), 84.4\left(\mathrm{q}, J_{C-F}=23.9 \mathrm{~Hz}, \mathrm{CH}\right), 61.6(\mathrm{CH}), 47.6$ $\left(\mathrm{CH}_{2}\right), 30.0\left(\mathrm{CH}_{2}\right), 19.8\left(\mathrm{CH}_{3}\right), 19.0\left(\mathrm{CH}_{2}\right), 13.6\left(\mathrm{CH}_{3}\right) .{ }^{1} \mathrm{H}$ NMR $\left(400 \mathrm{MHz}, \delta \mathrm{ppm} / \mathrm{CDCl}_{3}\right):$ : $24-$ $7.20(\mathrm{~m}, 3 \mathrm{H}), 7.03-7.01(\mathrm{~m}, 1 \mathrm{H}), 4.81-4.68(\mathrm{~m}, 2 \mathrm{H}), 3.77-3.69(\mathrm{~m}, 1 \mathrm{H}), 3.32-3.25(\mathrm{~m}, 1 \mathrm{H}), 2.33$ $(\mathrm{s}, 3 \mathrm{H}), 1.60-1.52(\mathrm{~m}, 2 \mathrm{H}), 1.36-1.27(\mathrm{~m}, 2 \mathrm{H}), 0.88(\mathrm{t}, J=7.2 \mathrm{~Hz}, 3 \mathrm{H}) .{ }^{19} \mathrm{~F}$ NMR $(376 \mathrm{MHz}, \delta$ ppm/ $\mathrm{CDCl}_{3}$ ): -73.4 (s). HRMS for $\mathrm{C}_{14} \mathrm{H}_{19} \mathrm{~F}_{3} \mathrm{~N}_{3}{ }^{+}$: calcd. $[\mathrm{M}+\mathrm{H}]^{+}: 286.1526$, found: 286.1519 . 
Compound 4o: 1-butyl-5-(2-methoxyphenyl)-4-(trifluoromethyl)-4,5-dihydro-1H-1,2,3triazole

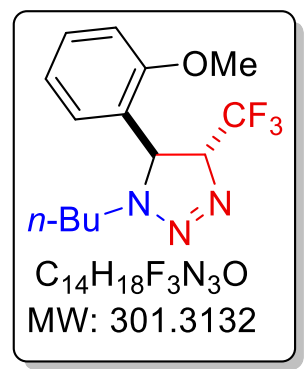

Following the general procedure, treatment of 2-methoxybenzaldehyde 10 (68 $\mathrm{mg}, 0.50 \mathrm{mmol}$ ), n-butylamine $2 \mathrm{a}$ (44 $\mathrm{mg}, 0.60 \mathrm{mmol}$ ) with trifluorodiazoethane stock solution 3 in toluene $(1.83 \mathrm{~mL}, 1.50 \mathrm{mmol})$ in the presence of $\mathrm{Ag}_{2} \mathrm{CO}_{3}$ ( $4 \mathrm{mg}, 0.015 \mathrm{mmol}$ ) at $25{ }^{\circ} \mathrm{C}$ for $8 \mathrm{~h}$ followed by column chromatography afforded the product 40 (134 mg, 89\%). Major isomer: colorless liquid, $\mathbf{R}_{f}$ (Ethyl acetate/Hexane: $\left.5 / 95\right)=0.30 .{ }^{13} \mathbf{C} \mathbf{~ N M R}$ $\left(100 \mathrm{MHz}, \delta \mathrm{ppm} / \mathrm{CDCl}_{3}\right): 157.4(\mathrm{C}), 130.4(\mathrm{CH}), 128.9(\mathrm{CH}), 125.0(\mathrm{C}), 124.1$ (q, $\left.J_{C-F}=277.1 \mathrm{~Hz}, \mathrm{C}\right), 121.1(\mathrm{CH}), 111.3(\mathrm{CH}), 82.8\left(\mathrm{q}, J_{C-F}=27.7 \mathrm{~Hz}, \mathrm{CH}\right), 56.6(\mathrm{CH}), 55.5\left(\mathrm{CH}_{3}\right)$, $47.2\left(\mathrm{CH}_{2}\right), 29.9\left(\mathrm{CH}_{2}\right), 19.7\left(\mathrm{CH}_{2}\right), 13.6\left(\mathrm{CH}_{3}\right) .{ }^{1} \mathrm{H}$ NMR $\left(400 \mathrm{MHz}, \delta \mathrm{ppm} / \mathrm{CDCl}_{3}\right):$ 7.26-7.23 (m, $1 \mathrm{H}), 6.99(\mathrm{dd}, J=15.0 \mathrm{~Hz}, 1.2 \mathrm{~Hz}, 1 \mathrm{H}), 6.89-6.83(\mathrm{~m}, 2 \mathrm{H}), 4.80-4.70(\mathrm{~m}, 2 \mathrm{H}), 3.73(\mathrm{~s}, 3 \mathrm{H}), 3.63-$ $3.57(\mathrm{~m}, 1 \mathrm{H}), 3.19-3.14(\mathrm{~m}, 1 \mathrm{H}), 1.49-1.42(\mathrm{~m}, 2 \mathrm{H}), 1.26-1.21(\mathrm{~m}, 2 \mathrm{H}), 0.78(\mathrm{t}, J=6.0 \mathrm{~Hz}, 3 \mathrm{H})$. ${ }^{19} \mathrm{~F}$ NMR $\left(376 \mathrm{MHz}, \delta \mathrm{ppm} / \mathrm{CDCl}_{3}\right):-73.9$ (s). HRMS for $\mathrm{C}_{14} \mathrm{H}_{19} \mathrm{~F}_{3} \mathrm{~N}_{3} \mathrm{O}^{+}$: calcd. $[\mathrm{M}+\mathrm{H}]^{+}: 302.1475$, found: 302.1468 .

\section{Compound 4p: 1-butyl-5-(2-fluorophenyl)-4-(trifluoromethyl)-4,5-dihydro-1H-1,2,3-triazole}

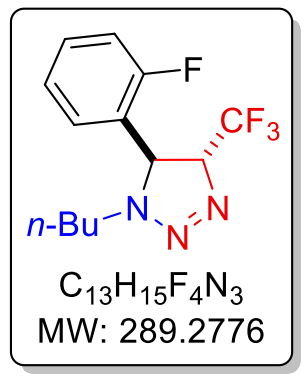

Following the general procedure, treatment of 3-fluorobenzaldehyde 1p (62 $\mathrm{mg}, 0.50 \mathrm{mmol}$ ), $n$-butylamine $2 \mathrm{a}(44 \mathrm{mg}, 0.60 \mathrm{mmol})$ with trifluorodiazoethane stock solution 3 in toluene $(1.83 \mathrm{~mL}, 1.50 \mathrm{mmol})$ in the presence of $\mathrm{Ag}_{2} \mathrm{CO}_{3}\left(4 \mathrm{mg}, 0.015 \mathrm{mmol}\right.$ ) at $25^{\circ} \mathrm{C}$ for $8 \mathrm{~h}$ followed by column chromatography afforded the product $4 p$ as a colorless liquid mixture of diastereomers (124 $\mathrm{mg}, 86 \%$ ). Major isomer: $\mathbf{R}_{f}$ (Ethyl acetate/Hexane: $3 / 97)=0.30 .{ }^{13} \mathrm{C} \mathrm{NMR}\left(100 \mathrm{MHz}, \delta \mathrm{ppm} / \mathrm{CDCl}_{3}\right): 160.7(\mathrm{~d}$, $\left.J_{C-F}=247.7 \mathrm{~Hz}, \mathrm{C}\right), 130.9(\mathrm{~d}, J=8.3 \mathrm{~Hz}, \mathrm{CH}), 128.7(\mathrm{~d}, J=3.1 \mathrm{~Hz}, \mathrm{CH}), 125.2(\mathrm{~d}, J=3.8 \mathrm{~Hz}, \mathrm{CH})$, $124.5(\mathrm{~d}, J=12.1 \mathrm{~Hz}, \mathrm{C}), 123.7\left(\mathrm{q}, J_{C-F}=277.0 \mathrm{~Hz}, \mathrm{C}\right), 116.3(\mathrm{~d}, J=21.1 \mathrm{~Hz}, \mathrm{CH}), 84.0\left(\mathrm{q}, J_{C-F}=\right.$ $27.5 \mathrm{~Hz}, \mathrm{CH}), 55.2(\mathrm{CH}), 47.8\left(\mathrm{CH}_{2}\right), 29.8\left(\mathrm{CH}_{2}\right), 19.8\left(\mathrm{CH}_{2}\right), 13.6\left(\mathrm{CH}_{3}\right) .{ }^{1} \mathrm{H}$ NMR $(400 \mathrm{MHz}, \delta$ $\left.\mathrm{ppm} / \mathrm{CDCl}_{3}\right)$ : 7.38-7.33 (m, $\left.1 \mathrm{H}\right), 7.19-7.11(\mathrm{~m}, 3 \mathrm{H}), 4.84-4.76(\mathrm{~m}, 2 \mathrm{H}), 3.73-3.66(\mathrm{~m}, 1 \mathrm{H}), 3.34-$ $3.28(\mathrm{~m}, 1 \mathrm{H}), 1.61-1.53(\mathrm{~m}, 2 \mathrm{H}), 1.36-1.26(\mathrm{~m}, 2 \mathrm{H}), 0.87(\mathrm{t}, J=7.2 \mathrm{~Hz}, 3 \mathrm{H}) .{ }^{19} \mathrm{~F} \mathrm{NMR}(376 \mathrm{MHz}$, $\left.\delta \mathrm{ppm} / \mathrm{CDCl}_{3}\right):-73.7\left(\mathrm{~d}, J=2.6 \mathrm{~Hz}\right.$ ), -119.0 (s). HRMS for $\mathrm{C}_{13} \mathrm{H}_{16} \mathrm{~F}_{4} \mathrm{~N}_{3}{ }^{+}$: calcd. $[\mathrm{M}+\mathrm{H}]^{+}: 290.1275$, found: 290.1272 .

Compound 4q: 1-butyl-5-(3,5-dimethoxyphenyl)-4-(trifluoromethyl)-4,5-dihydro-1H-1,2,3triazole

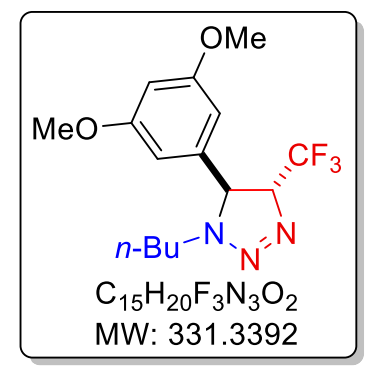

Following the general procedure, treatment of 4-hydroxybenzaldehyde 1q (83 mg, $0.50 \mathrm{mmol}$ ), $n$-butylamine 2a (44 mg, $0.60 \mathrm{mmol}$ ) with trifluorodiazoethane stock solution 3 in toluene $(1.83 \mathrm{~mL}, 1.50 \mathrm{mmol})$ in the presence of $\mathrm{Ag}_{2} \mathrm{CO}_{3}\left(4 \mathrm{mg}, 0.015 \mathrm{mmol}\right.$ ) at $25^{\circ} \mathrm{C}$ for $14 \mathrm{~h}$ followed by column chromatography afforded the product $\mathbf{4 q}(126 \mathrm{mg}, 76 \%)$. Major isomer: colorless liquid, $\mathbf{R}_{f}$ (Ethyl acetate/Hexane: $\left.5 / 95\right)=0.20$. ${ }^{13} \mathrm{C}$ NMR (100 MHz, $\left.\delta \mathrm{ppm} / \mathrm{CDCl}_{3}\right): 161.7$ (C), 161.7 (C), 140.2 (C), 123.8 
(q, $\left.J_{C-F}=221.2 \mathrm{~Hz}, \mathrm{C}\right), 104.9(\mathrm{CH}), 104.9(\mathrm{CH}), 100.7(\mathrm{CH}), 85.0\left(\mathrm{q}, J_{\mathrm{C}-F}=22.5 \mathrm{~Hz}, \mathrm{CH}\right), 61.7(\mathrm{CH})$, $55.6\left(\mathrm{CH}_{3}\right), 55.6\left(\mathrm{CH}_{3}\right), 47.9\left(\mathrm{CH}_{2}\right), 29.8\left(\mathrm{CH}_{2}\right), 19.9\left(\mathrm{CH}_{2}\right), 13.7\left(\mathrm{CH}_{3}\right) .{ }^{1} \mathbf{H}$ NMR $(400 \mathrm{MHz}, \delta$ $\left.\mathrm{ppm} / \mathrm{CDCl}_{3}\right): 6.43(\mathrm{t}, J=2.0 \mathrm{~Hz}, 1 \mathrm{H}), 6.31(\mathrm{~d}, J=2.4 \mathrm{~Hz}, 2 \mathrm{H}), 4.76-4.68(\mathrm{~m}, 1 \mathrm{H}), 4.42(\mathrm{~d}, J=10.4$ $\mathrm{Hz}, 1 \mathrm{H}), 3.78(\mathrm{~s}, 6 \mathrm{H}), 3.75-3.69(\mathrm{~m}, 1 \mathrm{H}), 3.38-3.12(\mathrm{~m}, 1 \mathrm{H}), 1.64-1.56(\mathrm{~m}, 2 \mathrm{H}), 1.38-1.29(\mathrm{~m}$, $2 \mathrm{H}), 0.90(\mathrm{t}, J=7.2 \mathrm{~Hz}, 3 \mathrm{H}) .{ }^{19} \mathrm{~F}$ NMR $\left(376 \mathrm{MHz}, \delta \mathrm{ppm} / \mathrm{CDCl}_{3}\right):-73.4$ (s). HRMS for $\mathrm{C}_{15} \mathrm{H}_{21} \mathrm{~F}_{3} \mathrm{~N}_{3} \mathrm{O}_{2}{ }^{+}$: calcd. [M+H] $]^{+}: 332.1580$, found: 332.1576 .

\section{Compound 4r: 5-(benzo[d][1,3]dioxol-5-yl)-1-butyl-4-(trifluoromethyl)-4,5-dihydro-1H- 1,2,3-triazole}

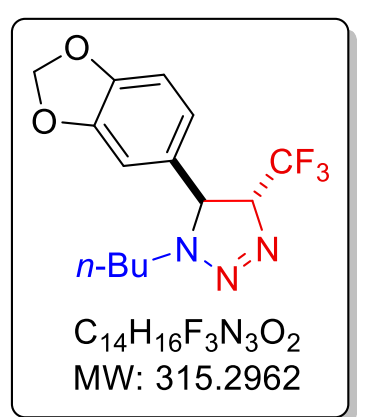

Following the general procedure, treatment of benzo[d][1,3]dioxole-5carbaldehyde 1r (75 mg, $0.50 \mathrm{mmol}$ ), $n$-butylamine 2a (44 mg, 0.60 $\mathrm{mmol}$ ) with trifluorodiazoethane stock solution 3 in toluene $(1.83 \mathrm{~mL}$, $1.50 \mathrm{mmol}$ ) in the presence of $\mathrm{Ag}_{2} \mathrm{CO}_{3}\left(4 \mathrm{mg}, 0.015 \mathrm{mmol}\right.$ ) at $25^{\circ} \mathrm{C}$ for $10 \mathrm{~h}$ followed by column chromatography afforded the product $\mathbf{4 r}$ (126 $\mathrm{mg}, 80 \%$ ). Major isomer: colorless liquid, $\mathbf{R}_{\boldsymbol{f}}$ (Ethyl acetate/Hexane: $5 / 95)=0.45 .{ }^{13} \mathrm{C} \mathrm{NMR}\left(100 \mathrm{MHz}, \delta \mathrm{ppm} / \mathrm{CDCl}_{3}\right): 148.8$ (C), 148.3 (C), $131.3(\mathrm{C}), 123.8\left(\mathrm{q}, J_{\mathrm{C}-F}=276.8 \mathrm{~Hz}, \mathrm{C}\right), 120.9(\mathrm{CH}), 108.9(\mathrm{CH}), 106.8$ $(\mathrm{CH}), 101.6\left(\mathrm{CH}_{2}\right), 84.8\left(\mathrm{q}, J_{\mathrm{C}-\mathrm{F}}=22.5 \mathrm{~Hz}, \mathrm{CH}\right), 61.6(\mathrm{~d}, J=1.8 \mathrm{~Hz}, \mathrm{CH}), 47.7\left(\mathrm{CH}_{2}\right), 29.7\left(\mathrm{CH}_{2}\right)$, $19.9\left(\mathrm{CH}_{2}\right), 13.6\left(\mathrm{CH}_{3}\right) .{ }^{1} \mathrm{H}$ NMR $\left(400 \mathrm{MHz}, \delta \mathrm{ppm} / \mathrm{CDCl}_{3}\right): 6.79(\mathrm{~d}, J=8.0 \mathrm{~Hz}, 1 \mathrm{H}), 6.67(\mathrm{dd}, J=$ $8.0 \mathrm{~Hz}, 1.6 \mathrm{~Hz}, 1 \mathrm{H}), 6.62(\mathrm{~d}, J=2.0 \mathrm{~Hz}, 1 \mathrm{H}), 5.98(\mathrm{~d}, J=1.6 \mathrm{~Hz}, 2 \mathrm{H}), 4.70-4.62(\mathrm{~m}, 1 \mathrm{H}), 4.42(\mathrm{~d}$, $J=10.0 \mathrm{~Hz}, 1 \mathrm{H}), 3.71-3.66(\mathrm{~m}, 1 \mathrm{H}), 3.34-3.28(\mathrm{~m}, 1 \mathrm{H}), 1.62-1.54(\mathrm{~m}, 2 \mathrm{H}), 1.36-1.27(\mathrm{~m}, 2 \mathrm{H})$, $0.89\left(\mathrm{t}, J=7.2 \mathrm{~Hz}, 3 \mathrm{H}\right.$ ). ${ }^{19} \mathrm{~F} \mathrm{NMR}\left(376 \mathrm{MHz}, \delta \mathrm{ppm} / \mathrm{CDCl}_{3}\right):-73.4$ (s). HRMS for $\mathrm{C}_{14} \mathrm{H}_{17} \mathrm{~F}_{3} \mathrm{~N}_{3} \mathrm{O}_{2}{ }^{+}$: calcd. $[\mathrm{M}+\mathrm{H}]^{+}:$316.1267, found: 316.1263 .

Compound 4s: 4-(1-butyl-4-(trifluoromethyl)-4,5-dihydro-1H-1,2,3-triazol-5-yl)-2(trifluoromethoxy)phenol

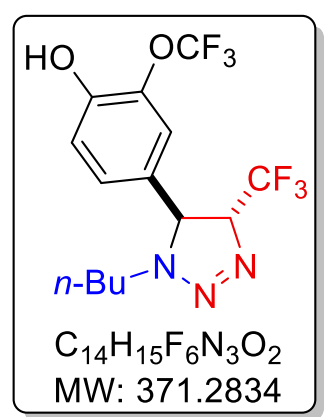

Following the general procedure, treatment of 4-hydroxy-3(trifluoromethoxy)benzaldehyde $1 \mathrm{~s}$ (103 $\mathrm{mg}, 0.50 \mathrm{mmol}$ ), $n$-butylamine 2a (44 $\mathrm{mg}, 0.60 \mathrm{mmol}$ ) with trifluorodiazoethane stock solution 3 in toluene $(1.83 \mathrm{~mL}, 1.50 \mathrm{mmol})$ in the presence of $\mathrm{Ag}_{2} \mathrm{CO}_{3}(4 \mathrm{mg}, 0.015$ $\mathrm{mmol}$ ) at $25^{\circ} \mathrm{C}$ for $8 \mathrm{~h}$ followed by column chromatography afforded the product 4s (139 $\mathrm{mg}, 75 \%$ ). Major isomer: white solid, $\mathbf{R}_{f}$ (Ethyl acetate/Hexane: $15 / 85)=0.25 . \mathrm{Mp} 123-125{ }^{\circ} \mathrm{C} .{ }^{13} \mathrm{C} \mathrm{NMR}(100 \mathrm{MHz}, \delta$ $\mathrm{ppm} / \mathrm{CDCl}_{3}$ ): 149.2 (C), 137.1 (C), 129.8 (C), 126.7 (CH), 123.6 (q, $J_{\mathrm{C}-\mathrm{F}}=276.9 \mathrm{~Hz}, \mathrm{C}$ ), 120.8 (q, $\left.J_{C-F}=257.7 \mathrm{~Hz}, \mathrm{C}\right), 120.4(\mathrm{CH}), 118.8(\mathrm{CH}), 84.5\left(\mathrm{q}, J_{C-F}=28.3 \mathrm{~Hz}, \mathrm{CH}\right), 61.3(\mathrm{CH}), 48.0\left(\mathrm{CH}_{2}\right)$, $29.8\left(\mathrm{CH}_{2}\right), 19.9\left(\mathrm{CH}_{2}\right), 13.6\left(\mathrm{CH}_{3}\right) .{ }^{1} \mathrm{H}$ NMR $\left(400 \mathrm{MHz}, \delta \mathrm{ppm} / \mathrm{CDCl}_{3}\right): 7.09-7.02(\mathrm{~m}, 3 \mathrm{H}), 6.63$ $(\mathrm{s}, 1 \mathrm{H}), 4.72-4.64(\mathrm{~m}, 1 \mathrm{H}), 4.48(\mathrm{~d}, J=10.4 \mathrm{~Hz}, 1 \mathrm{H}), 3.74-3.66(\mathrm{~m}, 1 \mathrm{H}), 3.38-3.32(\mathrm{~m}, 1 \mathrm{H}), 1.63-$ $1.55(\mathrm{~m}, 2 \mathrm{H}), 1.39-1.24(\mathrm{~m}, 2 \mathrm{H}), 0.89(\mathrm{t}, J=7.6 \mathrm{~Hz}, 3 \mathrm{H}) .{ }^{19} \mathrm{~F} \mathrm{NMR}\left(376 \mathrm{MHz}, \delta \mathrm{ppm} / \mathrm{CDCl}_{3}\right)$ : $58.1(\mathrm{~d}, J=6.8 \mathrm{~Hz}),-73.5(\mathrm{~d}, J=12.0 \mathrm{~Hz})$. HRMS for $\mathrm{C}_{14} \mathrm{H}_{16} \mathrm{~F}_{6} \mathrm{~N}_{3} \mathrm{O}_{2}{ }^{+}$: calcd. $[\mathrm{M}+\mathrm{H}]^{+}: 372.1141$, found: 372.1140 . 


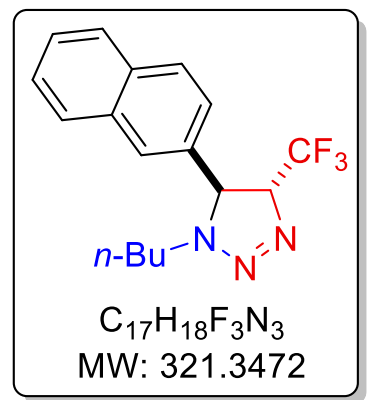

Following the general procedure, treatment of 2-naphthaldehyde $\mathbf{1 t}$ (78 $\mathrm{mg}, 0.50 \mathrm{mmol}$ ), $n$-butylamine $2 \mathrm{a}(44 \mathrm{mg}, 0.60 \mathrm{mmol}$ ) with trifluorodiazoethane stock solution 3 in toluene $(1.83 \mathrm{~mL}, 1.50 \mathrm{mmol})$ in the presence of $\mathrm{Ag}_{2} \mathrm{CO}_{3}(4 \mathrm{mg}, 0.015 \mathrm{mmol})$ at $25^{\circ} \mathrm{C}$ for $8 \mathrm{~h}$ followed by column chromatography afforded the product $4 \mathrm{t}(130 \mathrm{mg}, 81 \%)$. Major isomer: colorless liquid, $\mathbf{R}_{\boldsymbol{f}}$ (Ethyl acetate/Hexane: $\left.2 / 98\right)=0.20$. ${ }^{13} \mathrm{C} \mathrm{NMR}\left(100 \mathrm{MHz}, \delta \mathrm{ppm} / \mathrm{CDCl}_{3}\right): 134.9$ (C), 133.5 (C), 133.4 (C), 129.8 $(\mathrm{CH}), 128.0(\mathrm{CH}), 128.0(\mathrm{CH}), 127.1(\mathrm{CH}), 127.0(\mathrm{CH}), 126.7(\mathrm{CH}), 123.9\left(\mathrm{q}, J_{C-F}=276.8 \mathrm{~Hz}, \mathrm{C}\right)$, 123.7(CH), $85.0\left(q, J_{C-F}=28.1 \mathrm{~Hz}, \mathrm{CH}\right), 62.0(\mathrm{CH}), 47.9\left(\mathrm{CH}_{2}\right), 29.8\left(\mathrm{CH}_{2}\right), 19.9\left(\mathrm{CH}_{2}\right), 13.6\left(\mathrm{CH}_{3}\right)$. ${ }^{1} \mathrm{H}$ NMR $\left(400 \mathrm{MHz}, \delta \mathrm{ppm} / \mathrm{CDCl}_{3}\right): 7.92(\mathrm{~d}, J=8.8 \mathrm{~Hz}, 1 \mathrm{H}), 7.88-7.84(\mathrm{~m}, 2 \mathrm{H}), 7.72(\mathrm{~s}, 1 \mathrm{H}), 7.58-$ $7.52(\mathrm{~m}, 2 \mathrm{H}), 7.27(\mathrm{dd}, J=8.4 \mathrm{~Hz}, 2.0 \mathrm{~Hz}, 1 \mathrm{H}), 4.87-4.79(\mathrm{~m}, 1 \mathrm{H}), 4.71(\mathrm{~d}, J=10.4 \mathrm{~Hz}, 1 \mathrm{H}), 3.80-$ $3.72(\mathrm{~m}, 1 \mathrm{H}), 3.40-3.33(\mathrm{~m}, 1 \mathrm{H}), 1.66-1.58(\mathrm{~m}, 2 \mathrm{H}), 1.38-1.27(\mathrm{~m}, 2 \mathrm{H}), 0.89(\mathrm{t}, J=7.2 \mathrm{~Hz}, 3 \mathrm{H})$. ${ }^{19} \mathrm{~F}$ NMR (376 MHz, $\left.\delta \mathrm{ppm} / \mathrm{CDCl}_{3}\right):-73.3$ (s). HRMS for $\mathrm{C}_{17} \mathrm{H}_{19} \mathrm{~F}_{3} \mathrm{~N}_{3}{ }^{+}$: calcd. $[\mathrm{M}+\mathrm{H}]^{+}: 322.1526$, found: 322.1515 .

\section{Compound 4u: 1-butyl-5-ferrocenyl-4-(trifluoromethyl)-4,5-dihydro-1H-1,2,3-triazole}

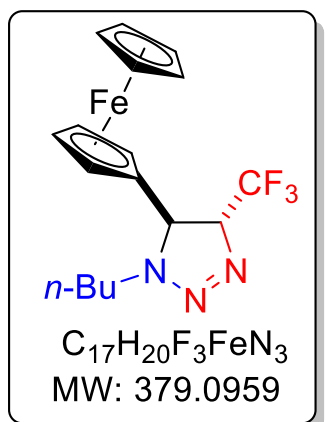

Following the general procedure, treatment of ferrocenecarboxaldehyde 1u (107 mg, $0.50 \mathrm{mmol}$ ), $n$-butylamine 2a (44 mg, $0.60 \mathrm{mmol}$ ) with trifluorodiazoethane stock solution 3 in toluene $(1.83 \mathrm{~mL}, 1.50 \mathrm{mmol})$ in the presence of $\mathrm{Ag}_{2} \mathrm{CO}_{3}\left(4 \mathrm{mg}, 0.015 \mathrm{mmol}\right.$ ) at $25^{\circ} \mathrm{C}$ for $8 \mathrm{~h}$ followed by column chromatography afforded the product $4 \mathbf{u}(178 \mathrm{mg}, 94 \%)$. Major isomer: brown viscous, $\mathbf{R}_{f}$ (Ethyl acetate/Hexane: $\left.5 / 95\right)=0.30 .{ }^{13} \mathbf{C}$ NMR $\left(100 \mathrm{MHz}, \delta \mathrm{ppm} / \mathrm{CDCl}_{3}\right): 124.2\left(\mathrm{q}, J_{C-F}=278.3 \mathrm{~Hz}, \mathrm{C}\right), 86.1(\mathrm{C}), 82.6\left(\mathrm{q}, J_{C-}\right.$ $F=27.6 \mathrm{~Hz}, \mathrm{CH}), 69.6(\mathrm{CH}), 68.9(\mathrm{CH}), 68.9(\mathrm{CH}), 68.9(\mathrm{CH}), 68.9(\mathrm{CH}), 68.9$

$(\mathrm{CH}), 68.6(\mathrm{CH}), 68.4(\mathrm{CH}), 65.1(\mathrm{CH}), 52.2(\mathrm{~d}, \mathrm{~J}=1.7 \mathrm{~Hz}, \mathrm{CH}), 46.7\left(\mathrm{CH}_{2}\right), 30.1\left(\mathrm{CH}_{2}\right), 19.8\left(\mathrm{CH}_{2}\right)$, 13.7 $\left(\mathrm{CH}_{3}\right) .{ }^{1} \mathrm{H}$ NMR $\left(400 \mathrm{MHz}, \delta \mathrm{ppm} / \mathrm{CDCl}_{3}\right): 5.20-5.13(\mathrm{~m}, 1 \mathrm{H}), 4.37(\mathrm{~d}, J=5.6 \mathrm{~Hz}, 1 \mathrm{H}), 4.24-$ $4.15(\mathrm{~m}, 8 \mathrm{H}), 3.88(\mathrm{~s}, 1 \mathrm{H}), 3.69-3.63(\mathrm{~m}, 1 \mathrm{H}), 3.26-3.19(\mathrm{~m}, 1 \mathrm{H}), 1.59-1.52(\mathrm{~m}, 2 \mathrm{H}), 1.36-1.28$ $(\mathrm{m}, 2 \mathrm{H}), 0.89(\mathrm{t}, J=7.2 \mathrm{~Hz}, 3 \mathrm{H}) .{ }^{19} \mathrm{~F}$ NMR $\left(376 \mathrm{MHz}, \delta \mathrm{ppm} / \mathrm{CDCl}_{3}\right):-74.1$ (s). HRMS for $\mathrm{C}_{17} \mathrm{H}_{21} \mathrm{~F}_{3} \mathrm{FeN}_{3}{ }^{+}$: calcd. [M+H] $]^{+}: 380.1032$, found: 380.1024 .

\section{Compound 4v: 1-butyl-5-(1H-pyrrol-2-yl)-4-(trifluoromethyl)-4,5-dihydro-1H-1,2,3-triazole}

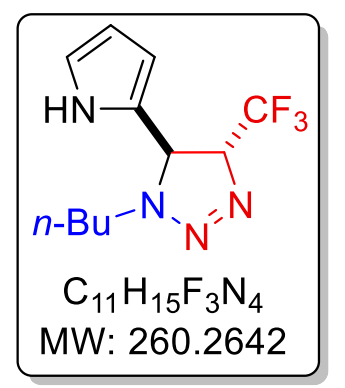

Following the general procedure, treatment $1 \mathrm{H}$-pyrrole-3-carbaldehyde 1v (48 $\mathrm{mg}, 0.50 \mathrm{mmol}), n$-butylamine $2 \mathrm{a}(44 \mathrm{mg}, 0.60 \mathrm{mmol}$ ) with trifluorodiazoethane stock solution 3 in toluene $(1.83 \mathrm{~mL}, 1.50 \mathrm{mmol})$ in the presence of $\mathrm{Ag}_{2} \mathrm{CO}_{3}(4 \mathrm{mg}, 0.015 \mathrm{mmol})$ at $25^{\circ} \mathrm{C}$ for $8 \mathrm{~h}$ followed by column chromatography afforded the product $4 \mathbf{v}$ (122 $\mathrm{mg}, 94 \%)$. Major isomer: white solid, $\mathbf{R}_{f}$ (Ethyl acetate/Hexane: $\left.5 / 95\right)=0.20 . \mathbf{M p} 95-97^{\circ} \mathrm{C}$. ${ }^{13} \mathrm{C} \mathrm{NMR}\left(100 \mathrm{MHz}, \delta \mathrm{ppm} / \mathrm{CDCl}_{3}\right): 125.9$ (C), 123.8 (q, $\left.J_{C-F}=277.0 \mathrm{~Hz}, \mathrm{C}\right)$, 
119.7 (CH), $109.8(\mathrm{CH}), 108.4(\mathrm{CH}), 81.0\left(\mathrm{q}, J_{C-F}=28.3 \mathrm{~Hz}, \mathrm{CH}\right), 55.9(\mathrm{CH}), 47.4\left(\mathrm{CH}_{2}\right), 29.9\left(\mathrm{CH}_{2}\right)$, $19.8\left(\mathrm{CH}_{2}\right), 13.6\left(\mathrm{CH}_{3}\right) .{ }^{1} \mathrm{H}$ NMR $\left(400 \mathrm{MHz}, \delta \mathrm{ppm} / \mathrm{CDCl}_{3}\right)$ : $9.88(\mathrm{~s}, 1 \mathrm{H}), 6.87(\mathrm{~s}, 1 \mathrm{H}), 6.25(1 \mathrm{H})$, 6.16-6.14 (m, 1H), $4.87(\mathrm{~s}, 1 \mathrm{H}), 4.73(\mathrm{~s}, 1 \mathrm{H}), 3.70-3.62(\mathrm{~m}, 1 \mathrm{H}), 3.40-3.33(\mathrm{~m}, 1 \mathrm{H}), 1.60-1.53$ $(\mathrm{m}, 2 \mathrm{H}), 1.38-1.25(\mathrm{~m}, 2 \mathrm{H}), 0.93-0.88(\mathrm{~m}, 3 \mathrm{H}) .{ }^{19} \mathrm{~F} \mathrm{NMR}\left(376 \mathrm{MHz}, \delta \mathrm{ppm} / \mathrm{CDCl}_{3}\right):-74.0(\mathrm{~d}, J=$ $5.6 \mathrm{~Hz}$ ). HRMS for $\mathrm{C}_{11} \mathrm{H}_{16} \mathrm{~F}_{3} \mathrm{~N}_{4}^{+}$: calcd. [M+H] $]^{+}: 261.1322$, found: 261.1309 .

\section{Compound 4w: 1-butyl-5-(furan-2-yl)-4-(trifluoromethyl)-4,5-dihydro-1H-1,2,3-triazole}

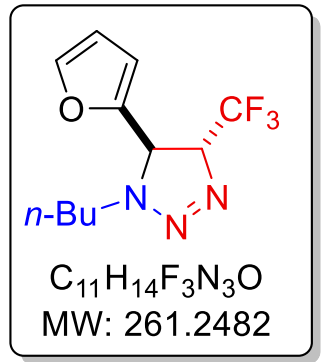

Following the general procedure, treatment of furan-2-carbaldehyde $\mathbf{1} \mathbf{w}$ (48 $\mathrm{mg}, 0.50 \mathrm{mmol}$ ), $n$-butylamine $2 \mathrm{a}$ (44 $\mathrm{mg}, 0.60 \mathrm{mmol}$ ) with trifluorodiazoethane stock solution 3 in toluene $(1.83 \mathrm{~mL}, 1.50 \mathrm{mmol})$ in the presence of $\mathrm{Ag}_{2} \mathrm{CO}_{3}\left(4 \mathrm{mg}, 0.015 \mathrm{mmol}\right.$ ) at $25^{\circ} \mathrm{C}$ for $14 \mathrm{~h}$ followed by column chromatography afforded the product $\mathbf{4 w}$ (88 $\mathrm{mg}, 67 \%$ ). Major isomer: colorless liquid, $\mathbf{R}_{\boldsymbol{f}}$ (Ethyl acetate/Hexane: $\left.2 / 98\right)=0.25 .{ }^{13} \mathrm{C}$ NMR $\left(100 \mathrm{MHz}, \delta \mathrm{ppm} / \mathrm{CDCl}_{3}\right): 148.7(\mathrm{C}), 143.9(\mathrm{CH}), 123.7$ (q, $J_{C-F}=276.8 \mathrm{~Hz}$, C), $110.9(\mathrm{CH}), 109.8(\mathrm{CH}), 81.0\left(\mathrm{q}, J_{\mathrm{C}-F}=28.6 \mathrm{~Hz}, \mathrm{CH}\right), 55.2(\mathrm{CH}), 47.8\left(\mathrm{CH}_{2}\right), 29.8\left(\mathrm{CH}_{2}\right), 19.8$ $\left(\mathrm{CH}_{2}\right), 13.6\left(\mathrm{CH}_{3}\right) .{ }^{1} \mathrm{H}$ NMR $\left(400 \mathrm{MHz}, \delta \mathrm{ppm} / \mathrm{CDCl}_{3}\right): 7.41(\mathrm{~s}, 1 \mathrm{H}), 6.37(\mathrm{~d}, J=1.2 \mathrm{~Hz}, 2 \mathrm{H}), 5.01-$ $4.93(\mathrm{~m}, 1 \mathrm{H}), 4.59(\mathrm{~d}, J=10.4 \mathrm{~Hz}, 1 \mathrm{H}), 3.67-3.60(\mathrm{~m}, 1 \mathrm{H}), 3.40-3.33(\mathrm{~m}, 1 \mathrm{H}), 1.59-1.52(\mathrm{~m}, 2 \mathrm{H})$, 1.37-1.26 (m, 2H), $0.88(\mathrm{t}, J=7.2 \mathrm{~Hz}, 3 \mathrm{H}) .{ }^{19} \mathrm{~F}$ NMR (376 MHz, $\left.\delta \mathrm{ppm} / \mathrm{CDCl}_{3}\right):-73.4$ (s). HRMS for $\mathrm{C}_{11} \mathrm{H}_{15} \mathrm{~F}_{3} \mathrm{~N}_{3} \mathrm{O}^{+}$: calcd. $[\mathrm{M}+\mathrm{H}]^{+}:$262.1162, found: 262.1159. Minor isomer: yellow liquid, $\mathbf{R}_{\boldsymbol{f}}$ (Ethyl acetate/Hexane: 5/95) = 0.30. ${ }^{13} \mathrm{C} \mathrm{NMR}\left(100 \mathrm{MHz}, \delta \mathrm{ppm} / \mathrm{CDCl}_{3}\right): 145.2(\mathrm{C}), 143.7(\mathrm{CH})$, $123.1\left(q, J_{C-F}=277.4 \mathrm{~Hz}, \mathrm{C}\right), 111.1(\mathrm{CH}), 110.8(\mathrm{CH}), 78.8\left(\mathrm{q}, J_{C-F}=28.5 \mathrm{~Hz}, \mathrm{CH}\right), 56.2(\mathrm{CH}), 47.8$ $\left(\mathrm{CH}_{2}\right), 30.4\left(\mathrm{CH}_{2}\right), 20.1\left(\mathrm{CH}_{2}\right), 13.8\left(\mathrm{CH}_{3}\right) .{ }^{1} \mathrm{H}$ NMR $\left(400 \mathrm{MHz}, \delta \mathrm{ppm} / \mathrm{CDCl}_{3}\right): 7.41-7.40(\mathrm{~m}, 1 \mathrm{H})$, 6.37-6.36 (m, 2H), $6.32(\mathrm{~d}, J=3.2 \mathrm{~Hz}, 1 \mathrm{H}), 4.91-4.80(\mathrm{~m}, 2 \mathrm{H}), 3.83-3.76(\mathrm{~m}, 1 \mathrm{H}), 3.16-3.09(\mathrm{~m}$, $1 \mathrm{H}), 1.65-1.60(\mathrm{~m}, 2 \mathrm{H}), 1.39-1.33(\mathrm{~m}, 2 \mathrm{H}), 0.91(\mathrm{t}, J=7.2 \mathrm{~Hz}, 3 \mathrm{H}) .{ }^{19} \mathrm{~F}$ NMR $(376 \mathrm{MHz}, \delta$ $\left.\mathrm{ppm} / \mathrm{CDCl}_{3}\right):-68.3(\mathrm{~s})$.

\section{Compound 4x: 1-butyl-5-(1H-imidazol-4-yl)-4-(trifluoromethyl)-4,5-dihydro-1H-1,2,3- triazole}

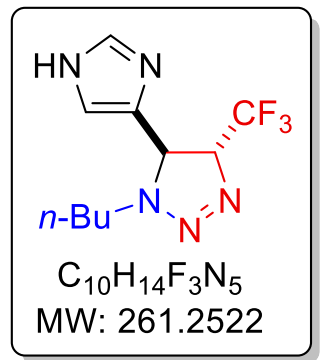

Following the general procedure, treatment of $1 \mathrm{H}$-imidazole-4carbaldehyde 1x (48 mg, $0.50 \mathrm{mmol}$ ), $n$-butylamine 2a (44 mg, 0.60 $\mathrm{mmol}$ ) with trifluorodiazoethane stock solution 3 in toluene $(1.83 \mathrm{~mL}$, $1.50 \mathrm{mmol}$ ) in the presence of $\mathrm{Ag}_{2} \mathrm{CO}_{3}(4 \mathrm{mg}, 0.015 \mathrm{mmol})$ at $25^{\circ} \mathrm{C}$ for 8 $\mathrm{h}$ followed by column chromatography afforded the product $4 \mathbf{x}(120 \mathrm{mg}$, 92\%). Major isomer: white solid, $\mathbf{R}_{\boldsymbol{f}}$ (Ethyl acetate/Hexane: 40/60) $=0.20$. Mp 124-126 ${ }^{\circ} \mathrm{C} .{ }^{13} \mathrm{C}$ NMR (100 MHz, $\delta$ ppm/CDCl $)$ ): 136.8 (CH), 135.9 (C), $124.0\left(\mathrm{q}, J_{C-F}=276.2 \mathrm{~Hz}, \mathrm{C}\right), 115.7(\mathrm{CH}), 81.7\left(\mathrm{q}, J_{C-F}=28.4 \mathrm{~Hz}, \mathrm{CH}\right), 55.6(\mathrm{~d}, J=1.5 \mathrm{~Hz}, \mathrm{CH})$, $47.4\left(\mathrm{CH}_{2}\right), 29.8\left(\mathrm{CH}_{2}\right), 19.8\left(\mathrm{CH}_{2}\right), 13.6\left(\mathrm{CH}_{3}\right) .{ }^{1} \mathrm{H} \mathrm{NMR}\left(400 \mathrm{MHz}, \delta \mathrm{ppm} / \mathrm{CDCl}_{3}\right): 7.67(\mathrm{~s}, 1 \mathrm{H})$, $7.08(\mathrm{~s}, 1 \mathrm{H}), 5.09-4.98(\mathrm{~m}, 1 \mathrm{H}), 4.68(\mathrm{~d}, J=10.0 \mathrm{~Hz}, 1 \mathrm{H}), 3.71-3.63(\mathrm{~m}, 1 \mathrm{H}), 3.37-3.30(\mathrm{~m}, 1 \mathrm{H})$, $1.62-1.53(\mathrm{~m}, 2 \mathrm{H}), 1.35-1.21(\mathrm{~m}, 2 \mathrm{H}), 0.86(\mathrm{t}, J=7.2 \mathrm{~Hz}, 3 \mathrm{H}) .{ }^{19} \mathrm{~F} \mathrm{NMR}\left(376 \mathrm{MHz}, \delta \mathrm{ppm} / \mathrm{CDCl}_{3}\right)$ : -73.5 (s). HRMS for $\mathrm{C}_{10} \mathrm{H}_{15} \mathrm{~F}_{3} \mathrm{~N}_{5}{ }^{+}$: calcd. [M+H] $]^{+}: 262.1274$, found: 262.1277 . 


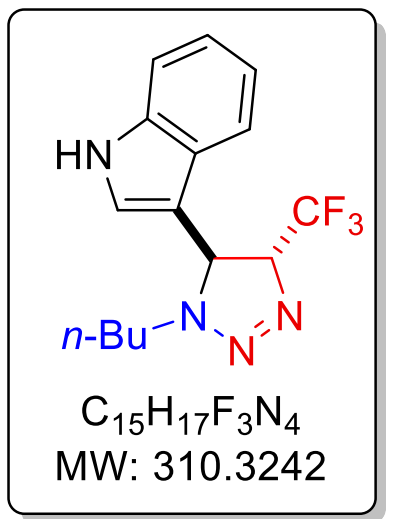

Following the general procedure, treatment of $1 \mathrm{H}$-indole-3carbaldehyde 1y (73 mg, $0.50 \mathrm{mmol}), n$-butylamine 2 a (44 mg, 0.60 $\mathrm{mmol})$ with trifluorodiazoethane stock solution 3 in toluene $(1.83 \mathrm{~mL}$, $1.50 \mathrm{mmol}$ ) in the presence of $\mathrm{Ag}_{2} \mathrm{CO}_{3}\left(4 \mathrm{mg}, 0.015 \mathrm{mmol}\right.$ ) at $25{ }^{\circ} \mathrm{C}$ for $14 \mathrm{~h}$ followed by column chromatography afforded the product 4y (120 mg, 77\%). Major isomer: white solid, $\mathbf{R}_{f}$ (Ethyl acetate/Hexane: $10 / 90)=0.30 . \mathrm{Mp} 117-119{ }^{\circ} \mathrm{C} .{ }^{13} \mathrm{C}$ NMR $(100 \mathrm{MHz}$, $\left.\delta \mathrm{ppm} / \mathrm{CDCl}_{3}\right): 137.1$ (C), $125.6(\mathrm{C}), 124.1\left(\mathrm{q}, J_{\mathrm{C}-\mathrm{F}}=276.9 \mathrm{~Hz}, \mathrm{C}\right), 123.8$ $(\mathrm{CH}), 123.2(\mathrm{CH}), 122.7(\mathrm{C}), 120.7(\mathrm{CH}), 118.8(\mathrm{CH}), 112.0(\mathrm{CH}), 81.9$ $\left(q, J_{C-F}=27.9 \mathrm{~Hz}, \mathrm{CH}\right), 55.0(\mathrm{CH}), 47.1\left(\mathrm{CH}_{2}\right), 30.0\left(\mathrm{CH}_{2}\right), 19.9\left(\mathrm{CH}_{2}\right), 13.7\left(\mathrm{CH}_{3}\right) .{ }^{1} \mathrm{H}$ NMR (400 $\left.\mathrm{MHz}, \delta \mathrm{ppm} / \mathrm{CDCl}_{3}\right): 8.53(\mathrm{~s}, 1 \mathrm{H}), 7.42(\mathrm{~d}, J=6.4 \mathrm{~Hz}, 1 \mathrm{H}), 7.33(\mathrm{~d}, J=6.4 \mathrm{~Hz}, 1 \mathrm{H}), 7.25-7.22(\mathrm{~m}$, $2 \mathrm{H}), 7.12(\mathrm{t}, J=6.0 \mathrm{~Hz}, 1 \mathrm{H}), 4.96-4.87(\mathrm{~m}, 2 \mathrm{H}), 3.73-3.67(\mathrm{~m}, 1 \mathrm{H}), 3.30-3.25(\mathrm{~m}, 1 \mathrm{H}), 1.61-1.55$ $(\mathrm{m}, 2 \mathrm{H}), 1.31-1.23(\mathrm{~m}, 2 \mathrm{H}), 0.84(\mathrm{t}, J=6.0 \mathrm{~Hz}, 3 \mathrm{H}) .{ }^{19} \mathrm{~F} \mathrm{NMR}\left(376 \mathrm{MHz}, \delta \mathrm{ppm} / \mathrm{CDCl}_{3}\right):-73.7(\mathrm{~s})$. HRMS for $\mathrm{C}_{15} \mathrm{H}_{18} \mathrm{~F}_{3} \mathrm{~N}_{4}^{+}$: calcd. [M+H] $]^{+}: 311.1478$, found: 311.1481 .

Compound 4z: 1-butyl-5-((E)-pent-1-en-1-yl)-4-(trifluoromethyl)-4,5-dihydro-1H-1,2,3triazole

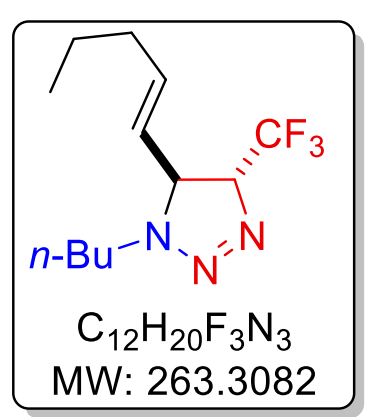

Following the general procedure, treatment of $(E)$-hex-2-enal $\mathbf{1 z}$ (49 $\mathrm{mg}, 0.50 \mathrm{mmol}$ ), $n$-butylamine $2 \mathrm{a}(44 \mathrm{mg}, 0.60 \mathrm{mmol}$ ) with trifluorodiazoethane stock solution 3 in toluene $(1.83 \mathrm{~mL}, 1.50 \mathrm{mmol})$ in the presence of $\mathrm{Ag}_{2} \mathrm{CO}_{3}\left(4 \mathrm{mg}, 0.015 \mathrm{mmol}\right.$ ) at $25{ }^{\circ} \mathrm{C}$ for $10 \mathrm{~h}$ followed by column chromatography afforded the product $4 \mathbf{z}(92 \mathrm{mg}$, 70\%). Major isomer: colorless liquid, $\mathbf{R}_{f}($ Ethyl acetate $/$ Hexane: $2 / 98)=$ 0.22. ${ }^{13} \mathrm{C}$ NMR (100 MHz, $\left.\delta \mathrm{ppm} / \mathrm{CDCl}_{3}\right): 138.2(\mathrm{CH}), 125.5(\mathrm{CH}), 123.9$ $\left(q, J_{C-F}=276.3 \mathrm{~Hz}, \mathrm{C}\right), 81.8\left(\mathrm{q}, J_{C-F}=28.1 \mathrm{~Hz}, \mathrm{CH}\right), 61.2(\mathrm{~d}, J=1.9 \mathrm{~Hz}, \mathrm{CH})$, $47.4\left(\mathrm{CH}_{2}\right), 34.2\left(\mathrm{CH}_{2}\right), 29.8\left(\mathrm{CH}_{2}\right), 22.0\left(\mathrm{CH}_{2}\right), 19.9\left(\mathrm{CH}_{2}\right), 13.6\left(\mathrm{CH}_{3}\right), 13.5\left(\mathrm{CH}_{3}\right) .{ }^{1} \mathrm{H}$ NMR (400 $\left.\mathrm{MHz}, \delta \mathrm{ppm} / \mathrm{CDCl}_{3}\right): 5.80-5.73(\mathrm{~m}, 1 \mathrm{H}), 5.27-5.21(\mathrm{~m}, 1 \mathrm{H}), 4.44-4.37(\mathrm{~m}, 1 \mathrm{H}), 3.94-3.89(\mathrm{~m}$, $1 \mathrm{H}), 3.62-3.54(\mathrm{~m}, 1 \mathrm{H}), 3.43-3.36(\mathrm{~m}, 1 \mathrm{H}), 2.04(\mathrm{q}, J=7.0 \mathrm{~Hz}, 2 \mathrm{H}), 1.66-1.59(\mathrm{~m}, 2 \mathrm{H}), 1.45-1.26$ $(\mathrm{m}, 4 \mathrm{H}), 0.93-0.86(\mathrm{~m}, 6 \mathrm{H}) .{ }^{19} \mathrm{~F}$ NMR $\left(376 \mathrm{MHz}, \delta \mathrm{ppm} / \mathrm{CDCl}_{3}\right):-72.8(\mathrm{~s})$. HRMS for $\mathrm{C}_{12} \mathrm{H}_{21} \mathrm{~F}_{3} \mathrm{~N}_{3}{ }^{+}$: calcd. $[\mathrm{M}+\mathrm{H}]^{+}:$264.1682, found: 264.1677 . 


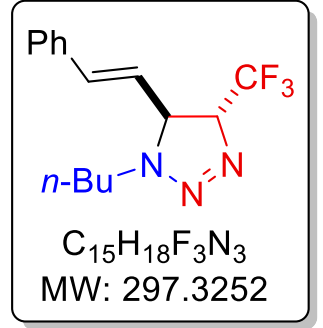

Following the general procedure, treatment of cinnamaldehyde 1aa (66 $\mathrm{mg}, \quad 0.50 \mathrm{mmol}), \quad n$-butylamine $2 \mathrm{a}(44 \mathrm{mg}, 0.60 \mathrm{mmol})$ with trifluorodiazoethane stock solution 3 in toluene $(1.83 \mathrm{~mL}, 1.50 \mathrm{mmol})$ in the presence of $\mathrm{Ag}_{2} \mathrm{CO}_{3}\left(4 \mathrm{mg}, 0.015 \mathrm{mmol}\right.$ ) at $25^{\circ} \mathrm{C}$ for $10 \mathrm{~h}$ followed by column chromatography afforded the product $4 \mathrm{aa}(134 \mathrm{mg}, 90 \%)$. Major isomer: colorless liquid, $\mathbf{R}_{f}$ (Ethyl acetate/Hexane: $2 / 98$ ) $=0.20 .{ }^{13} \mathbf{C}$ NMR $\left(100 \mathrm{MHz}, \delta \mathrm{ppm} / \mathrm{CDCl}_{3}\right): 136.0(\mathrm{CH}), 135.3(\mathrm{C}), 128.9(\mathrm{CH}), 128.9(\mathrm{CH}), 128.9(\mathrm{CH}), 126.8(\mathrm{CH})$, $126.8(\mathrm{CH}), 124.2(\mathrm{CH}), 123.8\left(\mathrm{q}, J_{C-F}=276.8 \mathrm{~Hz}, \mathrm{C}\right), 82.2\left(\mathrm{q}, J_{C-F}=28.1 \mathrm{~Hz}, \mathrm{CH}\right), 61.3(\mathrm{~d}, J=1.9$ $\mathrm{Hz}, \mathrm{CH}), 47.8\left(\mathrm{CH}_{2}\right), 29.9\left(\mathrm{CH}_{2}\right), 20.0\left(\mathrm{CH}_{2}\right), 13.7\left(\mathrm{CH}_{3}\right) .{ }^{1} \mathrm{H} \mathrm{NMR}\left(400 \mathrm{MHz}, \delta \mathrm{ppm} / \mathrm{CDCl}_{3}\right): 7.42-$ $7.32(\mathrm{~m}, 5 \mathrm{H}), 6.67(\mathrm{~d}, J=15.6 \mathrm{~Hz}, 1 \mathrm{H}), 5.99(\mathrm{q}, J=8.8 \mathrm{~Hz}, 1 \mathrm{H}), 4.64-4.56(\mathrm{~m}, 1 \mathrm{H}), 4.16(\mathrm{dd}, J=$ $11.2 \mathrm{~Hz}, 8.8 \mathrm{~Hz}, 1 \mathrm{H}), 3.72-3.64(\mathrm{~m}, 1 \mathrm{H}), 3.52-3.45(\mathrm{~m}, 1 \mathrm{H}), 1.73-1.66(\mathrm{~m}, 2 \mathrm{H}), 1.39-1.36(\mathrm{~m}$, $2 \mathrm{H}), 0.95(\mathrm{t}, J=7.2 \mathrm{~Hz}, 3 \mathrm{H}) .{ }^{19} \mathrm{~F} \mathrm{NMR}\left(376 \mathrm{MHz}, \delta \mathrm{ppm} / \mathrm{CDCl}_{3}\right):-72.6$ (s). HRMS for $\mathrm{C}_{15} \mathrm{H}_{19} \mathrm{~F}_{3} \mathrm{~N}_{3}{ }^{+}$: calcd. $[\mathrm{M}+\mathrm{H}]^{+}:$298.1526, found: 298.1526 .

\section{Compound 4ab: 1-butyl-5-cyclohexyl-4-(trifluoromethyl)-4,5-dihydro-1H-1,2,3-triazole}

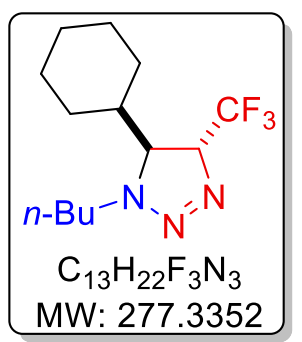

Following the general procedure, treatment of cyclohexanecarbaldehyde $1 \mathrm{ab}$ (56 mg, $0.50 \mathrm{mmol}$ ), $n$-butylamine 2a (44 mg, $0.60 \mathrm{mmol}$ ) with trifluorodiazoethane stock solution 3 in toluene $(1.83 \mathrm{~mL}, 1.50 \mathrm{mmol})$ in the presence of $\mathrm{Ag}_{2} \mathrm{CO}_{3}\left(4 \mathrm{mg}, 0.015 \mathrm{mmol}\right.$ ) at $25{ }^{\circ} \mathrm{C}$ for $8 \mathrm{~h}$ followed by column chromatography afforded the product $4 \mathrm{ab}$ (132 $\mathrm{mg}, 95 \%)$. Major isomer: colorless liquid, $\mathbf{R}_{f}$ (Ethyl acetate/Hexane: $\left.2 / 98\right)=0.20 .{ }^{13} \mathbf{C} \mathbf{~ N M R}$ (100 MHz, $\delta ~ p p m / \mathrm{CDCl}_{3}$ ): 124.0 (q, $\left.J_{C-F}=277.2 \mathrm{~Hz}, \mathrm{C}\right), 76.8$ (q, $J_{C-F}=27.8 \mathrm{~Hz}, \mathrm{CH}$ ), $60.6(\mathrm{CH})$, $47.4\left(\mathrm{CH}_{2}\right), 38.5\left(\mathrm{CH}_{2}\right), 30.0\left(\mathrm{CH}_{2}\right), 28.4\left(\mathrm{CH}_{2}\right), 26.3(\mathrm{CH}), 26.2\left(\mathrm{CH}_{2}\right), 26.0\left(\mathrm{CH}_{2}\right), 25.7\left(\mathrm{CH}_{2}\right), 19.8$ $\left(\mathrm{CH}_{2}\right), 13.6\left(\mathrm{CH}_{3}\right) .{ }^{1} \mathrm{H}$ NMR $\left(400 \mathrm{MHz}, \delta \mathrm{ppm} / \mathrm{CDCl}_{3}\right): 4.58-4.50(\mathrm{~m}, 1 \mathrm{H}), 3.90-3.82(\mathrm{~m}, 1 \mathrm{H}), 3.49-$ $3.46(\mathrm{~m}, 1 \mathrm{H}), 3.42-3.36(\mathrm{~m}, 1 \mathrm{H}), 1.81-1.52(\mathrm{~m}, 7 \mathrm{H}), 1.40-0.96(\mathrm{~m}, 7 \mathrm{H}), .92(\mathrm{t}, J=7.2 \mathrm{~Hz}, 3 \mathrm{H})$, 0.80-0.70 (m, 1H). ${ }^{19} \mathrm{~F} \mathrm{NMR} \mathrm{(376} \mathrm{MHz,} \delta \mathrm{ppm} / \mathrm{CDCl}_{3}$ ): -74.3 (s). HRMS for $\mathrm{C}_{13} \mathrm{H}_{23} \mathrm{~F}_{3} \mathrm{~N}_{3}{ }^{+}$: calcd. $[\mathrm{M}+\mathrm{H}]^{+}:$: 278.1839, found: 278.1832 .

\section{Compound 4ac: 1-benzyl-4-(1-butyl-4-(trifluoromethyl)-4,5-dihydro-1H-1,2,3-triazol-5- yl)piperidine}

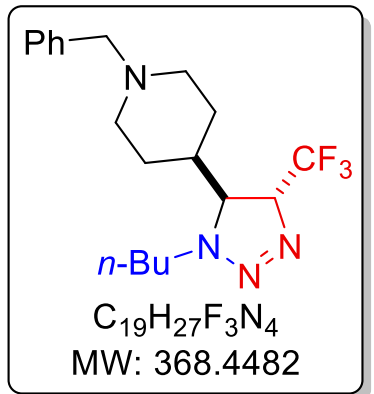

Following the general procedure, treatment of 1-benzylpiperidine-4carbaldehyde 1ac (102 mg, $0.50 \mathrm{mmol}$ ), $n$-butylamine 2 a (44 mg, 0.60 $\mathrm{mmol}$ ) with trifluorodiazoethane stock solution 3 in toluene $(1.83 \mathrm{~mL}$, $1.50 \mathrm{mmol}$ ) in the presence of $\mathrm{Ag}_{2} \mathrm{CO}_{3}$ (4 mg, $0.015 \mathrm{mmol}$ ) at $25^{\circ} \mathrm{C}$ for $8 \mathrm{~h}$ followed by column chromatography afforded the product 4 ac (146 mg, 79\%). Major isomer: white solid, $\mathbf{R}_{f}$ (Ethyl acetate/Hexane: 25/75) = 0.25. $\mathrm{Mp} \mathrm{90-92}{ }^{\circ} \mathrm{C} .{ }^{13} \mathrm{C}$ NMR (100 MHz, $\left.\delta \mathrm{ppm} / \mathrm{CDCl}_{3}\right): 138.2$ (C), $129.2(\mathrm{CH}), 129.2(\mathrm{CH}), 128.4(\mathrm{CH}), 128.4(\mathrm{CH}), 127.2(\mathrm{CH}), 123.9$ (q, $\left.J_{C-F}=277.3 \mathrm{~Hz}, \mathrm{C}\right), 77.5\left(\mathrm{q}, J_{C-F}=14.0 \mathrm{~Hz}, \mathrm{CH}\right), 63.2\left(\mathrm{CH}_{2}\right), 59.9(\mathrm{CH}), 53.2\left(\mathrm{CH}_{2}\right), 53.2\left(\mathrm{CH}_{2}\right)$, 
47.7 $\left(\mathrm{CH}_{2}\right), 37.2\left(\mathrm{CH}_{2}\right), 30.0\left(\mathrm{CH}_{2}\right), 30.0\left(\mathrm{CH}_{2}\right), 27.8\left(\mathrm{CH}_{2}\right), 26.0\left(\mathrm{CH}_{2}\right), 19.9\left(\mathrm{CH}_{2}\right), 13.7\left(\mathrm{CH}_{3}\right) .{ }^{1} \mathrm{H}$ NMR $\left(400 \mathrm{MHz}, \delta \mathrm{ppm} / \mathrm{CDCl}_{3}\right)$ : 7.33-7.23 $(\mathrm{m}, 5 \mathrm{H}), 4.67-4.59(\mathrm{~m}, 1 \mathrm{H}), 3.92-3.85(\mathrm{~m}, 1 \mathrm{H}), 3.53$ $(\mathrm{dd}, J=8.0 \mathrm{~Hz}, 4.0 \mathrm{~Hz}, 1 \mathrm{H}), 3.49(\mathrm{~J}=1.2 \mathrm{~Hz}, 2 \mathrm{H}), 3.43-3.37(\mathrm{~m}, 1 \mathrm{H}), 2.98-2.92(\mathrm{~m}, 2 \mathrm{H}), 1.98-$ $1.87(\mathrm{~m}, 2 \mathrm{H}), 1.70-1.59(\mathrm{~m}, 3 \mathrm{H}), 1.52-1.49(\mathrm{~m}, 1 \mathrm{H}), 1.40-1.29(\mathrm{~m}, 4 \mathrm{H}), 1.20-1.11(\mathrm{~m}, 1 \mathrm{H}), 0.96$ $\left(\mathrm{t}, J=7.6 \mathrm{~Hz}, 3 \mathrm{H}\right.$ ). ${ }^{19} \mathrm{~F}$ NMR $\left(376 \mathrm{MHz}, \delta \mathrm{ppm} / \mathrm{CDCl}_{3}\right.$ ): -74.1 (s). HRMS for $\mathrm{C}_{19} \mathrm{H}_{27} \mathrm{~F}_{3} \mathrm{~N}_{4}{ }^{+}$: calcd. $[\mathrm{M}+\mathrm{H}]^{+}:$369.2261, found: 369.2246 .

\section{Compound 4ad: 1-heptyl-5-phenyl-4-(trifluoromethyl)-4,5-dihydro-1H-1,2,3-triazole}

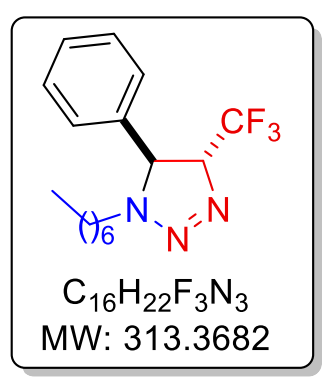

Following the general procedure, treatment of benzaldehyde 1a (53 mg, $0.50 \mathrm{mmol})$, heptan-1-amine $\mathbf{2 b}(69 \mathrm{mg}, 0.60 \mathrm{mmol})$ with trifluorodiazoethane stock solution 3 in toluene $(1.83 \mathrm{~mL}, 1.50 \mathrm{mmol})$ in the presence of $\mathrm{Ag}_{2} \mathrm{CO}_{3}\left(4 \mathrm{mg}, 0.015 \mathrm{mmol}\right.$ ) at $25^{\circ} \mathrm{C}$ for $8 \mathrm{~h}$ followed by column chromatography afforded the product $4 \mathrm{ad}(150 \mathrm{mg}, 96 \%)$. Major isomer: colorless liquid, $\mathbf{R}_{f}$ (Ethyl acetate/Hexane: $\left.2 / 98\right)=0.21 .{ }^{13} \mathbf{C}$ NMR (100 MHz, $\left.\delta ~ p p m / \mathrm{CDCl}_{3}\right): 137.7$ (C), $129.5(\mathrm{CH}), 129.5(\mathrm{CH}), 129.0(\mathrm{CH})$,

$127.0(\mathrm{CH}), 127.0(\mathrm{CH}), 123.8\left(\mathrm{q}, J_{C-F}=276.8 \mathrm{~Hz}, \mathrm{C}\right), 85.1\left(\mathrm{q}, J_{C-F}=28.1 \mathrm{~Hz}, \mathrm{CH}\right), 61.7\left(\mathrm{~d}, J_{C-F}=\right.$ $1.7 \mathrm{~Hz}, \mathrm{CH}), 48.1\left(\mathrm{CH}_{2}\right), 31.7\left(\mathrm{CH}_{2}\right), 28.8\left(\mathrm{CH}_{2}\right), 27.7\left(\mathrm{CH}_{2}\right), 26.6\left(\mathrm{CH}_{2}\right), 22.6\left(\mathrm{CH}_{2}\right), 14.1\left(\mathrm{CH}_{3}\right) .{ }^{1} \mathrm{H}$

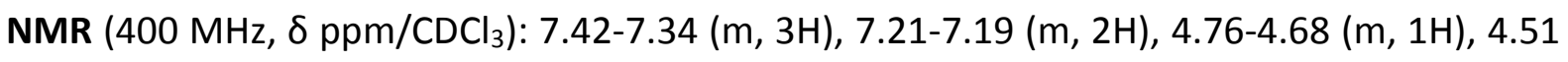
$(\mathrm{d}, J=10.4 \mathrm{~Hz}, 1 \mathrm{H}), 3.71-3.66(\mathrm{~m}, 1 \mathrm{H}), 3.36-3.29(\mathrm{~m}, 1 \mathrm{H}), 1.61-1.56(\mathrm{~m}, 2 \mathrm{H}), 1.31-1.15(\mathrm{~m}, 8 \mathrm{H})$, $0.86\left(\mathrm{t}, J=6.8 \mathrm{~Hz}, 3 \mathrm{H}\right.$ ). ${ }^{19} \mathrm{~F}$ NMR $\left(376 \mathrm{MHz}, \delta \mathrm{ppm} / \mathrm{CDCl}_{3}\right):-73.4(\mathrm{~s})$. HRMS for $\mathrm{C}_{16} \mathrm{H}_{23} \mathrm{~F}_{3} \mathrm{~N}_{3}{ }^{+}$: calcd. $[\mathrm{M}+\mathrm{H}]^{+}:$314.1839, found: 314.1828 .

\section{Compound 4ae: 1-dodecyl-5-phenyl-4-(trifluoromethyl)-4,5-dihydro-1H-1,2,3-triazole}

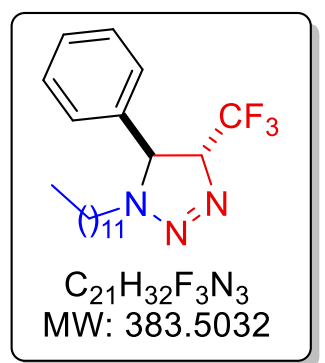

Following the general procedure, treatment of benzaldehyde 1 a $(53 \mathrm{mg}$, $0.50 \mathrm{mmol})$, dodecan-1-amine $2 \mathrm{c}(111 \mathrm{mg}, 0.60 \mathrm{mmol})$ with trifluorodiazoethane stock solution 3 in toluene $(1.83 \mathrm{~mL}, 1.50 \mathrm{mmol})$ in the presence of $\mathrm{Ag}_{2} \mathrm{CO}_{3}\left(4 \mathrm{mg}, 0.015 \mathrm{mmol}\right.$ ) at $25^{\circ} \mathrm{C}$ for $8 \mathrm{~h}$ followed by column chromatography afforded the product 4ae (159 mg, 83\%). Major isomer: colorless liquid, $\mathbf{R}_{f}$ (Ethyl acetate/Hexane: $\left.2 / 98\right)=0.24$. $\mathbf{M p} 38-40$ ${ }^{\circ} \mathrm{C} .{ }^{13} \mathrm{C}$ NMR (100 MHz, $\left.\delta \mathrm{ppm} / \mathrm{CDCl}_{3}\right): 137.8(\mathrm{C}), 129.5(\mathrm{CH}), 129.5(\mathrm{CH})$, $129.0(\mathrm{CH}), 127.0(\mathrm{CH}), 127.0(\mathrm{CH}), 123.8\left(\mathrm{q}, J_{\mathrm{C}-\mathrm{F}}=276.8 \mathrm{~Hz}, \mathrm{C}\right), 85.3-84.4(\mathrm{~m}, \mathrm{CH}), 61.7(\mathrm{CH})$, $48.1\left(\mathrm{CH}_{2}\right), 32.0\left(\mathrm{CH}_{2}\right), 29.7\left(\mathrm{CH}_{2}\right), 29.7\left(\mathrm{CH}_{2}\right), 29.6\left(\mathrm{CH}_{2}\right), 29.5\left(\mathrm{CH}_{2}\right), 29.4\left(\mathrm{CH}_{2}\right), 29.2\left(\mathrm{CH}_{2}\right)$, $27.7\left(\mathrm{CH}_{2}\right), 26.2\left(\mathrm{CH}_{2}\right), 22.8\left(\mathrm{CH}_{2}\right), 14.2\left(\mathrm{CH}_{3}\right) .{ }^{1} \mathrm{H}$ NMR $\left(400 \mathrm{MHz}, \delta \mathrm{ppm} / \mathrm{CDCl}_{3}\right):$ 7.42-7.34 (m, $3 \mathrm{H})$, 7.21-7.18 (m, 2H), 4.76-4.67 (m, $1 \mathrm{H}), 4.51(\mathrm{~d}, J=10.4 \mathrm{~Hz}, 1 \mathrm{H}), 3.71-3.66(\mathrm{~m}, 1 \mathrm{H}), 3.36-$ $3.29(\mathrm{~m}, 1 \mathrm{H}), 1.67-1.56(\mathrm{~m}, 2 \mathrm{H}), 1.38-1.24(\mathrm{~m}, 18 \mathrm{H}), 0.88(\mathrm{t}, J=7.2 \mathrm{~Hz}, 3 \mathrm{H}) .{ }^{19} \mathrm{~F} \mathrm{NMR}(376 \mathrm{MHz}$, $\delta \mathrm{ppm} / \mathrm{CDCl}_{3}$ ): -73.4 (s). HRMS for $\mathrm{C}_{21} \mathrm{H}_{33} \mathrm{~F}_{3} \mathrm{~N}_{3}{ }^{+}$: calcd. [M+H] $]^{+}: 384.2621$, found: 384.2620 . 


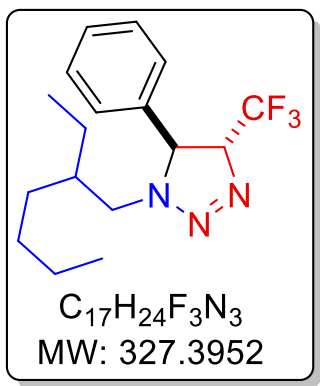

Following the general procedure, treatment of benzaldehyde $1 \mathrm{a}(53 \mathrm{mg}$, $0.50 \mathrm{mmol})$, 2-ethylhexan-1-amine $2 \mathrm{~d}(78 \mathrm{mg}, 0.60 \mathrm{mmol}$ ) with trifluorodiazoethane stock solution 3 in toluene $(1.83 \mathrm{~mL}, 1.50 \mathrm{mmol})$ in the presence of $\mathrm{Ag}_{2} \mathrm{CO}_{3}\left(4 \mathrm{mg}, 0.015 \mathrm{mmol}\right.$ ) at $25^{\circ} \mathrm{C}$ for $10 \mathrm{~h}$ followed by column chromatography afforded the product 4 af $(144 \mathrm{mg}, 88 \%)$. Major isomer: white solid, $\mathbf{R}_{f}$ (Ethyl acetate/Hexane: $\left.2 / 98\right)=0.25 .{ }^{13} \mathbf{C ~ N M R}$ (100 $\mathrm{MHz}, \delta \mathrm{ppm} / \mathrm{CDCl}_{3}$ ): 137.7 (d, $\left.J_{\mathrm{C}-\mathrm{F}}=8.0 \mathrm{~Hz}, \mathrm{C}\right), 129.5(\mathrm{CH}), 129.5(\mathrm{CH})$, $129.1(\mathrm{CH}), 127.0(\mathrm{CH}), 127.0(\mathrm{CH}), 123.8\left(\mathrm{q}, J_{C-F}=277.0 \mathrm{~Hz}, \mathrm{C}\right), 85.3-84.4(\mathrm{~m}, \mathrm{CH}), 62.2\left(\mathrm{~d}, J_{C-F}\right.$ $=8.0 \mathrm{~Hz}, \mathrm{CH}), 51.2\left(\mathrm{~d}, J_{C-F}=15.7 \mathrm{~Hz}, \mathrm{CH}_{2}\right), 37.9\left(\mathrm{~d}, J_{C-F}=24.6 \mathrm{~Hz}, \mathrm{CH}\right), 30.6\left(\mathrm{~d}, J_{C-F}=5.7 \mathrm{~Hz}, \mathrm{CH}_{2}\right)$, $28.5\left(\mathrm{~d}, J_{C-F}=46.7 \mathrm{~Hz}, \mathrm{CH}_{2}\right), 23.8\left(\mathrm{~d}, J_{C-F}=29.9 \mathrm{~Hz}, \mathrm{CH}_{2}\right), 22.9\left(\mathrm{~d}, J_{\mathrm{C}-F}=7.7 \mathrm{~Hz}, \mathrm{CH}_{2}\right), 14.0\left(\mathrm{CH}_{3}\right)$, $10.4\left(\mathrm{~d}, J_{C-F}=61.0 \mathrm{~Hz}, \mathrm{CH}_{3}\right) .{ }^{1} \mathrm{H}$ NMR $\left(400 \mathrm{MHz}, \delta \mathrm{ppm} / \mathrm{CDCl}_{3}\right): 7.41-7.35(\mathrm{~m}, 3 \mathrm{H}), 7.18-7.15(\mathrm{~m}$, $2 \mathrm{H}), 4.80-4.72(\mathrm{~m}, 1 \mathrm{H}), 4.51(\mathrm{~d}, J=9.2 \mathrm{~Hz}, 1 \mathrm{H}), 3.63-3.56(\mathrm{~m}, 1 \mathrm{H}), 3.26-3.18(\mathrm{~m}, 1 \mathrm{H}), 1.64-1.58$ $(\mathrm{m}, 2 \mathrm{H}), 1.39-1.09(\mathrm{~m}, 8 \mathrm{H}), 0.87-0.75(\mathrm{~m}, 6 \mathrm{H}) .{ }^{19} \mathrm{~F} \mathrm{NMR}\left(376 \mathrm{MHz}, \delta \mathrm{ppm} / \mathrm{CDCl}_{3}\right):-73.7(\mathrm{~d}, J=$ 4.9 Hz), (s). HRMS for $\mathrm{C}_{17} \mathrm{H}_{25} \mathrm{~F}_{3} \mathrm{~N}_{3}{ }^{+}$: calcd. $[\mathrm{M}+\mathrm{H}]^{+}: 328.1995$, found: 328.1993 .

Compound 4ag: 1-(cyclohexylmethyl)-5-phenyl-4-(trifluoromethyl)-4,5-dihydro-1H-1,2,3triazole

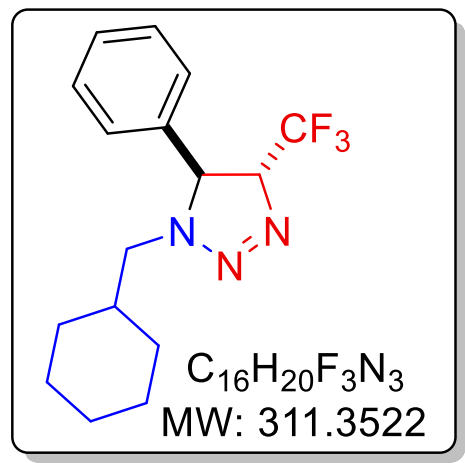

Following the general procedure, treatment of benzaldehyde 1a (53 $\mathrm{mg}, 0.50 \mathrm{mmol}$ ), cyclohexylmethanamine $2 \mathrm{e}(68 \mathrm{mg}, 0.60$ $\mathrm{mmol}$ ) with trifluorodiazoethane stock solution 3 in toluene $(1.83 \mathrm{~mL}, 1.50 \mathrm{mmol})$ in the presence of $\mathrm{Ag}_{2} \mathrm{CO}_{3}(4 \mathrm{mg}, 0.015$ $\mathrm{mmol}$ ) at $25{ }^{\circ} \mathrm{C}$ for $8 \mathrm{~h}$ followed by column chromatography afforded the product $4 \mathrm{ag}$ (139 $\mathrm{mg}, 89 \%)$. Major isomer: viscous compound, $\mathbf{R}_{\boldsymbol{f}}$ (Ethyl acetate/Hexane: $\left.2 / 98\right)=0.25 .{ }^{13} \mathrm{C}$ NMR (100 $\left.\mathrm{MHz}, \delta \mathrm{ppm} / \mathrm{CDCl}_{3}\right): 137.7(\mathrm{C}), 129.5(\mathrm{CH}), 129.5(\mathrm{CH}), 129.1(\mathrm{CH})$, $127.0(\mathrm{CH}), 127.0(\mathrm{CH}), 123.8\left(\mathrm{q}, J_{C-F}=277.1 \mathrm{~Hz}, \mathrm{C}\right), 84.9\left(\mathrm{q}, J_{C-F}=28.1 \mathrm{~Hz}, \mathrm{CH}\right), 62.3(\mathrm{CH}), 54.1$ $\left(\mathrm{CH}_{2}\right), 36.4(\mathrm{CH}), 30.9\left(\mathrm{CH}_{2}\right), 30.6\left(\mathrm{CH}_{2}\right), 26.4\left(\mathrm{CH}_{2}\right), 25.8\left(\mathrm{CH}_{2}\right), 25.7\left(\mathrm{CH}_{2}\right) .{ }^{1} \mathrm{H}$ NMR $(400 \mathrm{MHz}$, $\left.\delta \mathrm{ppm} / \mathrm{CDCl}_{3}\right):$ 7.42-7.34 (m, 3H), 7.91-7.16 (m, 2H), 4.77-4.69 (m, $\left.1 \mathrm{H}\right), 4.52(\mathrm{~d}, J=9.6 \mathrm{~Hz}, 1 \mathrm{H})$, $3.55-3.50(\mathrm{~m}, 1 \mathrm{H}), 3.14(\mathrm{dd}, J=14.0 \mathrm{~Hz}, 5.6 \mathrm{~Hz}, 1 \mathrm{H}), 1.72-1.58(\mathrm{~m}, 6 \mathrm{H}), 1.25-1.10(\mathrm{~m}, 3 \mathrm{H}), 0.95-$ $0.83(\mathrm{~m}, 2 \mathrm{H}) .{ }^{19} \mathrm{~F}$ NMR $\left(376 \mathrm{MHz}, \delta \mathrm{ppm} / \mathrm{CDCl}_{3}\right):-73.5$ (s). HRMS for $\mathrm{C}_{16} \mathrm{H}_{21} \mathrm{~F}_{3} \mathrm{~N}_{3}{ }^{+}$: calcd. [M+H] $]^{+}$: 312.1682, found: 312.1686. Minor isomer: white solid, $\mathbf{R}_{f}$ (Ethyl acetate/Hexane: 5/95) = 0.25. Mp 90-92 ${ }^{\circ} \mathrm{C} .{ }^{13} \mathrm{C} \mathrm{NMR}\left(100 \mathrm{MHz}, \delta \mathrm{ppm} / \mathrm{CDCl}_{3}\right): 131.3$ (C), $129.2(\mathrm{CH}), 129.2(\mathrm{CH}), 128.7(\mathrm{CH})$, $128.7(\mathrm{CH}), 128.7(\mathrm{CH}), 123.2\left(\mathrm{q}, J_{C-F}=277.6 \mathrm{~Hz}, \mathrm{C}\right), 80.0$ (q, $J_{C-F}=27.8 \mathrm{~Hz}, \mathrm{CH}$ ), $63.3(\mathrm{CH}), 53.9$ $\left(\mathrm{CH}_{2}\right), 37.1(\mathrm{CH}), 31.1\left(\mathrm{CH}_{2}\right), 30.9\left(\mathrm{CH}_{2}\right), 26.4\left(\mathrm{CH}_{2}\right), 25.9\left(\mathrm{CH}_{2}\right), 25.8\left(\mathrm{CH}_{2}\right) .{ }^{1} \mathrm{H}$ NMR $(400 \mathrm{MHz}$, $\left.\delta \mathrm{ppm} / \mathrm{CDCl}_{3}\right):$ 7.35-7.33 (m, 3H), $7.10(\mathrm{~s}, 2 \mathrm{H}), 5.03-4.93(\mathrm{~m}, 1 \mathrm{H}), 4.82(\mathrm{~d}, J=13.2 \mathrm{~Hz}, 1 \mathrm{H}), 3.69$ (dd, $J=13.6 \mathrm{~Hz}, 8.4 \mathrm{~Hz}, 1 \mathrm{H}), 2.97(\mathrm{dd}, J=14.0 \mathrm{~Hz}, 5.6 \mathrm{~Hz}, 1 \mathrm{H}), 1.77-1.61(\mathrm{~m}, 6 \mathrm{H}), 1.28-1.12(\mathrm{~m}$, $3 \mathrm{H}), 0.99-0.86(\mathrm{~m}, 2 \mathrm{H}) .{ }^{19} \mathrm{~F}$ NMR $\left(376 \mathrm{MHz}, \delta \mathrm{ppm} / \mathrm{CDCl}_{3}\right):-66.1(\mathrm{~s})$. 


\section{Compound 4ah: 1-benzyl-5-phenyl-4-(trifluoromethyl)-4,5-dihydro-1H-1,2,3-triazole}

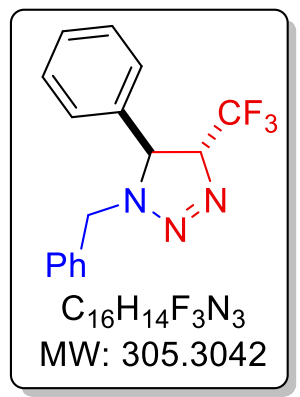

Following the general procedure, treatment of benzaldehyde $1 \mathrm{a}$ (53 mg, $0.50 \mathrm{mmol})$, phenylmethanamine $2 \mathrm{f}(64 \mathrm{mg}, 0.60 \mathrm{mmol})$ with trifluorodiazoethane stock solution 3 in toluene $(1.83 \mathrm{~mL}, 1.50 \mathrm{mmol})$ in the presence of $\mathrm{Ag}_{2} \mathrm{CO}_{3}$ ( $4 \mathrm{mg}, 0.015 \mathrm{mmol}$ ) at $25{ }^{\circ} \mathrm{C}$ for $9 \mathrm{~h}$ followed by column chromatography afforded the product $4 \mathrm{ah}$ (128 $\mathrm{mg}, 84 \%)$. Major isomer: colorless liquid, $\mathbf{R}_{f}$ (Ethyl acetate/Hexane: $2 / 98$ ) $=0.22 .{ }^{13} \mathbf{C}$ NMR $\left(100 \mathrm{MHz}, \delta \mathrm{ppm} / \mathrm{CDCl}_{3}\right): 137.1$ (C), 134.3 (C), 129.5 (CH), 129.5 (CH), 129.1 (CH), $129.0(\mathrm{CH}), 129.0(\mathrm{CH}), 128.5(\mathrm{CH}), 128.5(\mathrm{CH}), 128.4(\mathrm{CH}), 127.3(\mathrm{CH}), 127.3(\mathrm{CH}), 123.7$ $\left(q, J_{C-F}=276.8 \mathrm{~Hz}, \mathrm{C}\right), 85.5\left(\mathrm{q}, J_{C-F}=28.3 \mathrm{~Hz}, \mathrm{CH}\right), 60.3(\mathrm{~d}, J=1.7 \mathrm{~Hz}, \mathrm{CH}), 51.8\left(\mathrm{CH}_{2}\right) .{ }^{1} \mathrm{H}$ NMR (400 MHz, $\left.\delta p p m / \mathrm{CDCl}_{3}\right):$ 7.42-7.31 (m, 6H), 7.16-7.11 (m, 4H), $5.3(\mathrm{~d}, J=15.2 \mathrm{~Hz}, 1 \mathrm{H}), 4.77-$ $4.73(\mathrm{~m}, 1 \mathrm{H}), 4.28(\mathrm{~d}, J=10.8 \mathrm{~Hz}, 1 \mathrm{H}), 4.22(\mathrm{~d}, J=15.2 \mathrm{~Hz}, 1 \mathrm{H}) .{ }^{19} \mathrm{~F}$ NMR $(376 \mathrm{MHz}, \delta$ ppm/CDCl $)$ : -73.1 (s). HRMS for $\mathrm{C}_{16} \mathrm{H}_{15} \mathrm{~F}_{3} \mathrm{~N}_{3}{ }^{+}$: calcd. [M+H] ${ }^{+}$: 306.1213, found: 306.1210.

\section{Compound 4ai: 1-phenethyl-5-phenyl-4-(trifluoromethyl)-4,5-dihydro-1H-1,2,3-triazole}

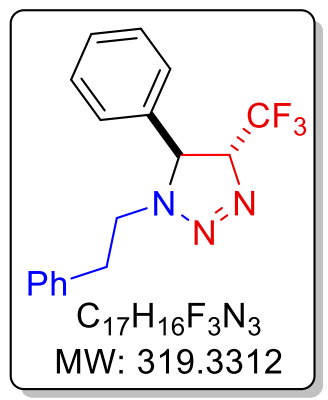

Following the general procedure, treatment of benzaldehyde $1 \mathrm{a}(53 \mathrm{mg}$, $0.50 \mathrm{mmol}), 2$-phenylethan-1-amine $2 \mathrm{~g}(73 \mathrm{mg}, 0.60 \mathrm{mmol})$ with trifluorodiazoethane stock solution 3 in toluene $(1.83 \mathrm{~mL}, 1.50 \mathrm{mmol})$ in the presence of $\mathrm{Ag}_{2} \mathrm{CO}_{3}$ ( $4 \mathrm{mg}, 0.015 \mathrm{mmol}$ ) at $25^{\circ} \mathrm{C}$ for $12 \mathrm{~h}$ followed by column chromatography afforded the product 4ai (121 mg, 76\%). Major isomer: colorless liquid, $\mathbf{R}_{\boldsymbol{f}}$ (Ethyl acetate/Hexane: $\left.5 / 95\right)=0.35 .{ }^{13} \mathbf{C} \mathbf{N M R}$ (100 MHz, $\left.\delta p p m / \mathrm{CDCl}_{3}\right): 138.2$ (C), $137.4(\mathrm{C}), 129.5(\mathrm{CH}), 129.5(\mathrm{CH})$, $129.1(\mathrm{CH}), 128.9(\mathrm{CH}), 128.9(\mathrm{CH}), 128.8(\mathrm{CH}), 128.8(\mathrm{CH}), 127.1(\mathrm{CH}), 127.1(\mathrm{CH}), 126.9(\mathrm{CH})$, $123.7\left(\mathrm{q}, J_{C-F}=276.9 \mathrm{~Hz}, \mathrm{C}\right), 85.2\left(\mathrm{q}, J_{C-F}=28.2 \mathrm{~Hz}, \mathrm{CH}\right), 62.2(\mathrm{CH}), 49.4\left(\mathrm{CH}_{2}\right), 34.9\left(\mathrm{CH}_{2}\right) .{ }^{1} \mathrm{H}$ NMR $\left(400 \mathrm{MHz}, \delta \mathrm{ppm} / \mathrm{CDCl}_{3}\right):$ 7.37-7.33 (m, 3H), 7.31-7.21 (m, 3H), 7.13-7.11 (m, 2H), 7.07$7.02(\mathrm{~m}, 2 \mathrm{H}), 4.73-4.65(\mathrm{~m}, 1 \mathrm{H}), 4.36(\mathrm{~d}, J=10.4 \mathrm{~Hz}, 1 \mathrm{H}), 3.97-3.93(\mathrm{~m}, 1 \mathrm{H}), 3.57-3.50(\mathrm{~m}, 1 \mathrm{H})$,

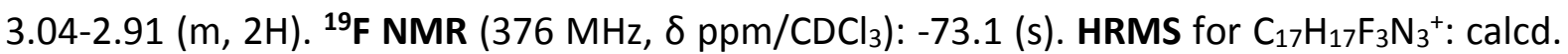
$[\mathrm{M}+\mathrm{H}]^{+}: 320.1369$, found: 320.1366 .

Compound 4aj: 1-(2,2-diphenylethyl)-5-phenyl-4-(trifluoromethyl)-4,5-dihydro-1H-1,2,3triazole

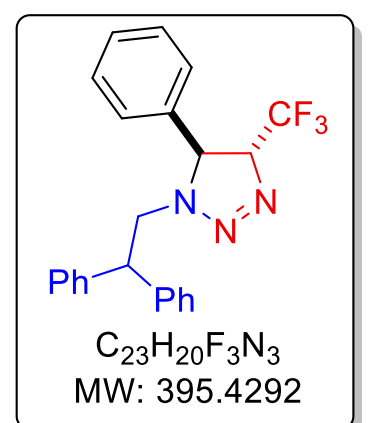

$(\mathrm{CH}), 128.8(\mathrm{CH}), 128.8(\mathrm{CH}), 128.3(\mathrm{CH}), 128.3(\mathrm{CH}), 128.0(\mathrm{CH}), 128.0(\mathrm{CH}), 127.2(\mathrm{CH}), 127.2$

Following the general procedure, treatment of benzaldehyde 1a (53 $\mathrm{mg}, 0.50 \mathrm{mmol}$ ), 2,2-diphenylethan-1-amine $2 \mathrm{~h}(118 \mathrm{mg}, 0.60 \mathrm{mmol})$ with trifluorodiazoethane stock solution 3 in toluene $(1.83 \mathrm{~mL}, 1.50$ $\mathrm{mmol}$ ) in the presence of $\mathrm{Ag}_{2} \mathrm{CO}_{3}\left(4 \mathrm{mg}, 0.015 \mathrm{mmol}\right.$ ) at $25^{\circ} \mathrm{C}$ for $9 \mathrm{~h}$ followed by column chromatography afforded the product 4aj (156 $\mathrm{mg}, 79 \%)$. Major isomer: white solid, $\mathbf{R}_{\boldsymbol{f}}$ (Ethyl acetate/Hexane: $\left.2 / 98\right)=$

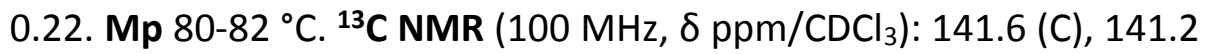
(C), $137.2(\mathrm{C}), 129.5(\mathrm{CH}), 129.5(\mathrm{CH}), 129.1(\mathrm{CH}), 128.9(\mathrm{CH}), 128.9$ 
$(\mathrm{CH}), 127.0(\mathrm{CH}), 127.0(\mathrm{CH}), 123.5\left(\mathrm{q}, J_{C-F}=277.0 \mathrm{~Hz}, \mathrm{C}\right), 85.2\left(\mathrm{q}, J_{C-F}=28.2 \mathrm{~Hz}, \mathrm{CH}\right), 62.2(\mathrm{CH})$, $52.6(\mathrm{CH}), 50.5\left(\mathrm{CH}_{2}\right) .{ }^{1} \mathrm{H}$ NMR $\left(400 \mathrm{MHz}, \delta \mathrm{ppm} / \mathrm{CDCl}_{3}\right)$ : 7.37-7.25 $(\mathrm{m}, 8 \mathrm{H}), 7.23-7.17(\mathrm{~m}, 5 \mathrm{H})$, 6.96-6.94 (m, 2H), 4.66-4.58 (m, 1H), 4.45-4.35 (m, 2H), $4.11(\mathrm{~d}, J=10.4 \mathrm{~Hz}, 1 \mathrm{H}), 3.97-3.87(\mathrm{~m}$, 1H). ${ }^{19} \mathrm{~F}$ NMR (376 MHz, $\left.\delta \mathrm{ppm} / \mathrm{CDCl}_{3}\right):-73.3$ (s). HRMS for $\mathrm{C}_{23} \mathrm{H}_{21} \mathrm{~F}_{3} \mathrm{~N}_{3}{ }^{+}$: calcd. $[\mathrm{M}+\mathrm{H}]^{+}$: 396.1682, found: 396.1688 .

Compound 4ak: 1-(furan-2-ylmethyl)-5-phenyl-4-(trifluoromethyl)-4,5-dihydro-1H-1,2,3triazole

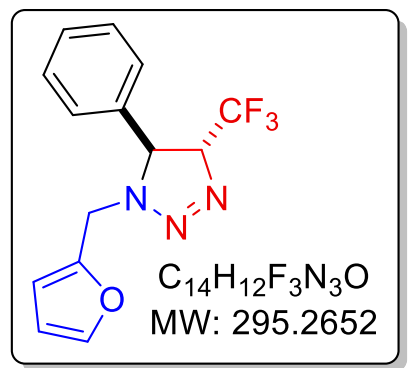

Following the general procedure, treatment of benzaldehyde 1a (53 $\mathrm{mg}, 0.50 \mathrm{mmol}$ ), furan-2-ylmethanamine $2 \mathbf{i}(58 \mathrm{mg}, 0.60 \mathrm{mmol}$ ) with trifluorodiazoethane stock solution 3 in toluene $(1.83 \mathrm{~mL}, 1.50$ $\mathrm{mmol}$ ) in the presence of $\mathrm{Ag}_{2} \mathrm{CO}_{3}$ ( $4 \mathrm{mg}, 0.015 \mathrm{mmol}$ ) at $25^{\circ} \mathrm{C}$ for 8 $\mathrm{h}$ followed by column chromatography afforded the product 4 ak (102 $\mathrm{mg}$, 69\%). Major isomer: colorless liquid, $\mathbf{R}_{f}$ (Ethyl acetate/Hexane: 5/95) $=0.26 .{ }^{13} \mathrm{C}$ NMR $\left(100 \mathrm{MHz}, \delta \mathrm{ppm} / \mathrm{CDCl}_{3}\right)$ : $148.3(\mathrm{C}), 143.2(\mathrm{CH}), 136.9(\mathrm{C}), 129.4(\mathrm{CH}), 129.4(\mathrm{CH}), 129.1(\mathrm{CH}), 127.2(\mathrm{CH}), 127.2(\mathrm{CH})$, $123.6\left(\mathrm{q}, J_{C-F}=276.7 \mathrm{~Hz}, \mathrm{C}\right), 110.5(\mathrm{CH}), 109.7(\mathrm{CH}), 85.5\left(\mathrm{q}, J_{C-F}=28.4 \mathrm{~Hz}, \mathrm{CH}\right), 60.8(\mathrm{~d}, J=1.8$ $\mathrm{Hz}, \mathrm{CH}), 44.8\left(\mathrm{CH}_{2}\right) .{ }^{1} \mathrm{H}$ NMR (400 MHz, $\left.\delta \mathrm{ppm} / \mathrm{CDCl}_{3}\right)$ : 7.42-7.35 (m, 4H), 7.22-7.20 (m, $\left.2 \mathrm{H}\right)$, $6.30(\mathrm{dd}, J=3.2 \mathrm{~Hz}, 2.0,1 \mathrm{H}), 6.18(\mathrm{~d}, J=3.2 \mathrm{~Hz}, 1 \mathrm{H}), 5.14(\mathrm{~d}, J=1.5 \mathrm{~Hz}, 1 \mathrm{H}), 4.73(\mathrm{q}, J=7.2 \mathrm{~Hz}$, 1H), 4.37-4.32 (m, 2H). ${ }^{19} \mathrm{~F}$ NMR (376 MHz, $\left.\delta \mathrm{ppm} / \mathrm{CDCl}_{3}\right):-72.9$ (s). HRMS for $\mathrm{C}_{14} \mathrm{H}_{13} \mathrm{~F}_{3} \mathrm{~N}_{3} \mathrm{O}^{+}$: calcd. $[\mathrm{M}+\mathrm{H}]^{+}:$296.1005, found: 296.1012 .

\section{Compound 4al: 1-isopropyl-5-phenyl-4-(trifluoromethyl)-4,5-dihydro-1H-1,2,3-triazole}

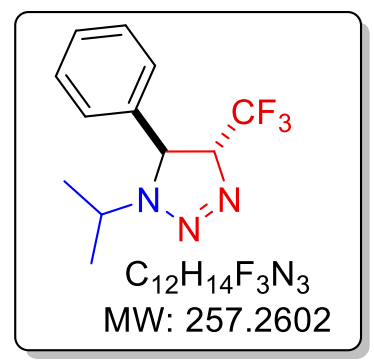

Following the general procedure, treatment of benzaldehyde 1a (53 $\mathrm{mg}, 0.50 \mathrm{mmol})$, isopropylamine $2 \mathrm{j}(36.0 \mathrm{mg}, 0.60 \mathrm{mmol})$ with trifluorodiazoethane stock solution 3 in toluene $(1.83 \mathrm{~mL}, 1.50 \mathrm{mmol})$ in the presence of $\mathrm{Ag}_{2} \mathrm{CO}_{3}(4 \mathrm{mg}, 0.015 \mathrm{mmol})$ at $25^{\circ} \mathrm{C}$ for $9 \mathrm{~h}$ followed by column chromatography afforded the product 4 al (109 $\mathrm{mg}, 85 \%)$.

Major isomer: white solid, $\mathbf{R}_{f}$ (Ethyl acetate/Hexane: $\left.2 / 98\right)=0.23$. $\mathbf{M p}$ 40-42 ${ }^{\circ} \mathrm{C} .{ }^{13} \mathrm{C}$ NMR $\left(100 \mathrm{MHz}, \delta \mathrm{ppm} / \mathrm{CDCl}_{3}\right): 138.9(\mathrm{C}), 129.4(\mathrm{CH}), 129.4(\mathrm{CH}), 128.9(\mathrm{CH})$, $126.9(\mathrm{CH}), 126.9(\mathrm{CH}), 123.8\left(\mathrm{q}, J_{C-F}=276.6 \mathrm{~Hz}, \mathrm{C}\right), 85.0$ (q, $\left.J_{C-F}=28.0 \mathrm{~Hz}, \mathrm{CH}\right), 60.5$ (d, J = 1.5 $\mathrm{Hz}, \mathrm{CH}), 50.6(\mathrm{CH}), 21.3\left(\mathrm{CH}_{3}\right), 20.6\left(\mathrm{CH}_{3}\right) .{ }^{1} \mathrm{H} \mathrm{NMR}\left(400 \mathrm{MHz}, \delta \mathrm{ppm} / \mathrm{CDCl}_{3}\right): 7.38-7.31(\mathrm{~m}, 3 \mathrm{H})$, 7.23-7.21 (m, 2H), 4.69-4.61 (m, 1H), $4.53(\mathrm{~d}, J=10.4 \mathrm{~Hz}, 1 \mathrm{H}), 3.76-3.36(\mathrm{~m}, 1 \mathrm{H}), 1.42(\mathrm{~d}, J=$ $6.8 \mathrm{~Hz}, 3 \mathrm{H}$ ), 1.15 (d, $J=6.4 \mathrm{~Hz}, 3 \mathrm{H}$ ). ${ }^{19} \mathrm{~F} \mathrm{NMR}$ (376 MHz, $\left.\delta \mathrm{ppm} / \mathrm{CDCl}_{3}\right):-73.5$ (s). HRMS for $\mathrm{C}_{12} \mathrm{H}_{15} \mathrm{~F}_{3} \mathrm{~N}_{3}{ }^{+}$: calcd. [M+H] $]^{+}: 258.1213$, found: 258.1208 . 


\section{Compound 4am: 1-cyclohexyl-5-phenyl-4-(trifluoromethyl)-4,5-dihydro-1H-1,2,3-triazole}

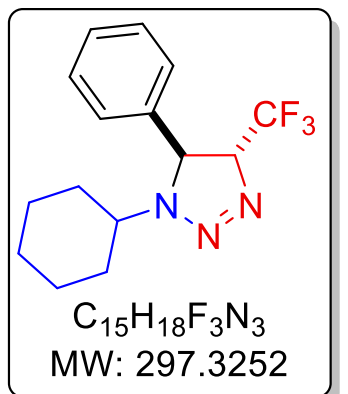

Following the general procedure, treatment of benzaldehyde 1a (53 $\mathrm{mg}, 0.50 \mathrm{mmol})$, cyclohexanamine $2 \mathrm{k}(60 \mathrm{mg}, 0.60 \mathrm{mmol})$ with trifluorodiazoethane stock solution 3 in toluene $(1.83 \mathrm{~mL}, 1.50 \mathrm{mmol})$ in the presence of $\mathrm{Ag}_{2} \mathrm{CO}_{3}\left(4 \mathrm{mg}, 0.015 \mathrm{mmol}\right.$ ) at $25^{\circ} \mathrm{C}$ for $9 \mathrm{~h}$ followed by column chromatography afforded the product $4 \mathrm{am}$ (104 mg, 70\%). Major isomer: colorless liquid, $\mathbf{R}_{\boldsymbol{f}}$ (Ethyl acetate/Hexane: $\left.2 / 98\right)=0.22$. ${ }^{13} \mathrm{C}$ NMR (100 MHz, $\left.\delta \mathrm{ppm} / \mathrm{CDCl}_{3}\right): 139.1(\mathrm{C}), 129.5(\mathrm{CH}), 129.5(\mathrm{CH})$, $128.9(\mathrm{CH}), 126.9(\mathrm{CH}), 126.9(\mathrm{CH}), 123.9\left(\mathrm{q}, J_{C-F}=276.9 \mathrm{~Hz}, \mathrm{C}\right), 84.9\left(\mathrm{q}, J_{C-F}=27.9 \mathrm{~Hz}, \mathrm{CH}\right), 60.3$ $(\mathrm{CH}), 58.3(\mathrm{CH}), 31.8\left(\mathrm{CH}_{2}\right), 31.5\left(\mathrm{CH}_{2}\right), 25.5\left(\mathrm{CH}_{2}\right), 25.5\left(\mathrm{CH}_{2}\right), 25.3\left(\mathrm{CH}_{2}\right) .{ }^{1} \mathrm{H}$ NMR $(400 \mathrm{MHz}, \delta$ $\left.\mathrm{ppm} / \mathrm{CDCl}_{3}\right)$ : 7.41-7.33 (m, 3H), 7.21-7.18 (m, 2H), 4.68-4.57 (m, 2H), 3.39-3.32 (m, $\left.1 \mathrm{H}\right), 1.97-$ $1.79(\mathrm{~m}, 4 \mathrm{H}), 1.76-1.72(\mathrm{~m}, 1 \mathrm{H}), 1.63-1.60(\mathrm{~m}, 1 \mathrm{H}), 1.28-1.15(\mathrm{~m}, 4 \mathrm{H}) .{ }^{19} \mathrm{~F}$ NMR $(376 \mathrm{MHz}, \delta$ ppm/CDCl $)$ : -73.7 (s). HRMS for $\mathrm{C}_{15} \mathrm{H}_{19} \mathrm{~F}_{3} \mathrm{~N}_{3}{ }^{+}$: calcd. [M+H] ${ }^{+}:$298.1526, found: 298.1522.

Compound 4an: 5-phenyl-4-(trifluoromethyl)-4,5-dihydro-1H-1,2,3-triazol-1-yl)propan-1-ol

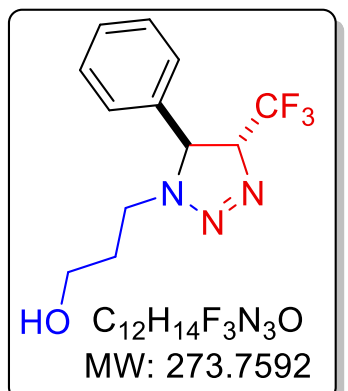

Following the general procedure, treatment of benzaldehyde 1a (53 $\mathrm{mg}, 0.50 \mathrm{mmol}$ ), 3-aminopropan-1-ol 21 (45 $\mathrm{mg}, 0.60 \mathrm{mmol}$ ) with trifluorodiazoethane stock solution 3 in toluene $(1.83 \mathrm{~mL}, 1.50 \mathrm{mmol})$ in the presence of $\mathrm{Ag}_{2} \mathrm{CO}_{3}\left(4 \mathrm{mg}, 0.015 \mathrm{mmol}\right.$ ) at $25^{\circ} \mathrm{C}$ for $14 \mathrm{~h}$ followed by column chromatography afforded the product 4 an $(75 \mathrm{mg}, 55 \%)$. Major isomer: colorless liquid, $\mathbf{R}_{\boldsymbol{f}}$ (Ethyl acetate/Hexane: $\left.30 / 70\right)=0.35$. ${ }^{13} \mathrm{C}$ NMR $\left(100 \mathrm{MHz}, \delta \mathrm{ppm} / \mathrm{CDCl}_{3}\right): 137.5(\mathrm{C}), 129.6(\mathrm{CH}), 129.6(\mathrm{CH})$, $129.2(\mathrm{CH}), 127.0(\mathrm{CH}), 127.0(\mathrm{CH}), 123.8\left(\mathrm{q}, J_{C-F}=221.3 \mathrm{~Hz}, \mathrm{C}\right), 85.0\left(\mathrm{q}, J_{C-F}=22.7 \mathrm{~Hz}, \mathrm{CH}\right), 62.1$ (d, $J=1.5 \mathrm{~Hz}, \mathrm{CH}), 59.5\left(\mathrm{CH}_{2}\right), 44.9\left(\mathrm{CH}_{2}\right), 30.6\left(\mathrm{CH}_{2}\right) .{ }^{1} \mathrm{H}$ NMR $\left(400 \mathrm{MHz}, \delta \mathrm{ppm} / \mathrm{CDCl}_{3}\right): 7.42-$ $7.36(\mathrm{~m}, 3 \mathrm{H}), 7.21-7.19(\mathrm{~m}, 2 \mathrm{H}), 4.78-4.70(\mathrm{~m}, 1 \mathrm{H}), 4.54(\mathrm{~d}, J=10.4 \mathrm{~Hz}, 1 \mathrm{H}), 3.86-3.78(\mathrm{~m}, 1 \mathrm{H})$,

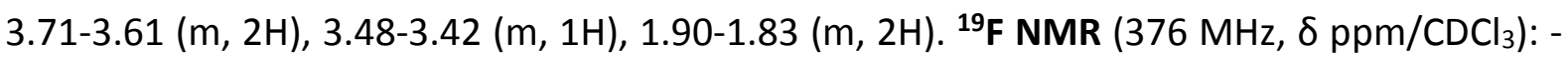
73.3 (s). HRMS for $\mathrm{C}_{12} \mathrm{H}_{15} \mathrm{~F}_{3} \mathrm{~N}_{3} \mathrm{O}^{+}$: calcd. [M+H] $]^{+}: 274.1162$, found: 274.1160 .

Compound 4ao: 1-(2,2-dimethoxyethyl)-5-phenyl-4-(trifluoromethyl)-4,5-dihydro-1H-1,2,3triazole

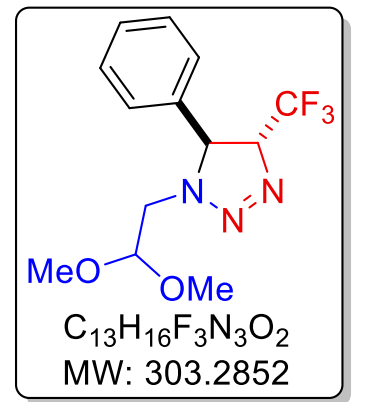

Following the general procedure, treatment of benzaldehyde $1 \mathbf{a}(53 \mathrm{mg}$, $0.50 \mathrm{mmol}$ ), 2,2-dimethoxyethan-1-amine $2 \mathrm{~m}$ (63 $\mathrm{mg}, 0.60 \mathrm{mmol}$ ) with trifluorodiazoethane stock solution 3 in toluene $(1.83 \mathrm{~mL}, 1.50 \mathrm{mmol})$ in the presence of $\mathrm{Ag}_{2} \mathrm{CO}_{3}$ ( $4 \mathrm{mg}, 0.015 \mathrm{mmol}$ ) at $25{ }^{\circ} \mathrm{C}$ for $8 \mathrm{~h}$ followed by column chromatography afforded the product 4 ao (118 mg, 78\%). Major isomer: colorless liquid, $\mathbf{R}_{f}$ (Ethyl acetate/Hexane: $\left.5 / 95\right)=0.30$. ${ }^{13} \mathrm{C}$ NMR (100 MHz, $\left.\delta \mathrm{ppm} / \mathrm{CDCl}_{3}\right): 137.7$ (C), $129.4(\mathrm{CH}), 129.4(\mathrm{CH})$, $129.0(\mathrm{CH}), 127.1(\mathrm{CH}), 127.1(\mathrm{CH}), 123.7\left(\mathrm{q}, J_{\mathrm{C}-F}=277.0 \mathrm{~Hz}, \mathrm{C}\right), 103.1(\mathrm{CH}), 85.3\left(\mathrm{q}, J_{C-F}=28.1\right.$ $\mathrm{Hz}, \mathrm{CH}), 61.8(\mathrm{~d}, J=7.2 \mathrm{~Hz}, \mathrm{CH}), 54.8\left(\mathrm{CH}_{3}\right), 54.8\left(\mathrm{CH}_{3}\right), 48.8\left(\mathrm{CH}_{2}\right) .{ }^{1} \mathbf{H}$ NMR $(400 \mathrm{MHz}, \delta$ $\left.\mathrm{ppm} / \mathrm{CDCl}_{3}\right): 7.40-7.32(\mathrm{~m}, 3 \mathrm{H}), 7.17(\mathrm{~d}, J=1.6 \mathrm{~Hz}, 2 \mathrm{H}), 4.80-4.69(\mathrm{~m}, 2 \mathrm{H}), 4.49(\mathrm{dd}, J=6.4 \mathrm{~Hz}$, 
$4.4 \mathrm{~Hz}, 1 \mathrm{H}), 3.97(\mathrm{dd}, J=14.8 \mathrm{~Hz}, 4.0 \mathrm{~Hz}, 1 \mathrm{H}), 3.37-3.29(\mathrm{~m}, 7 \mathrm{H}) .{ }^{19} \mathrm{~F}$ NMR $(376 \mathrm{MHz}, \delta$ ppm/ $\mathrm{CDCl}_{3}$ ): -73.7 (s). HRMS for $\mathrm{C}_{13} \mathrm{H}_{17} \mathrm{~F}_{3} \mathrm{~N}_{3} \mathrm{O}_{2}{ }^{+}$: calcd. [M+H]+: 304.1267, found: 304.1269 . Minor isomer: yellow liquid, $\mathbf{R}_{\boldsymbol{f}}$ (Ethyl acetate/Hexane: $\left.5 / 95\right)=0.23 .{ }^{13} \mathrm{C} \mathbf{N M R}(100 \mathrm{MHz}, \delta$ $\mathrm{ppm} / \mathrm{CDCl}_{3}$ ): $131.2(\mathrm{C}), 129.2(\mathrm{CH}), 128.7(\mathrm{CH}), 128.7(\mathrm{CH}), 128.7(\mathrm{CH}), 128.7(\mathrm{CH}), 123.1\left(\mathrm{q}, J_{C}\right.$ $F=277.7 \mathrm{~Hz}, \mathrm{C}), 102.9(\mathrm{CH}), 80.5\left(\mathrm{q}, J_{\mathrm{C}-\mathrm{F}}=27.8 \mathrm{~Hz}, \mathrm{CH}\right), 62.8(\mathrm{CH}), 54.3\left(\mathrm{CH}_{3}\right), 54.1\left(\mathrm{CH}_{3}\right), 48.4$ $\left(\mathrm{CH}_{2}\right) .{ }^{1} \mathrm{H}$ NMR $\left(400 \mathrm{MHz}, \delta \mathrm{ppm} / \mathrm{CDCl}_{3}\right): 7.35-7.33(\mathrm{~m}, 3 \mathrm{H}), 7.09(\mathrm{~s}, 2 \mathrm{H}), 5.05-4.98(\mathrm{~m}, 2 \mathrm{H})$, 4.56-4.54 (m, 1H), $4.12(\mathrm{dd}, J=14.8 \mathrm{~Hz}, 4.0 \mathrm{~Hz}, 1 \mathrm{H}), 3.33(\mathrm{~s}, 3 \mathrm{H}), 3.32(\mathrm{~s}, 3 \mathrm{H}), 3.23(\mathrm{dd}, J=14.8$ $\mathrm{Hz}, 6.0 \mathrm{~Hz}, 1 \mathrm{H}) .{ }^{19} \mathrm{~F}$ NMR $\left(376 \mathrm{MHz}, \delta \mathrm{ppm} / \mathrm{CDCl}_{3}\right):-66.1$ (s).

\section{Compound 4ap: N,N-dimethyl-2-(5-phenyl-4-(trifluoromethyl)-4,5-dihydro-1H-1,2,3-} triazol-1-yl)ethan-1-amine

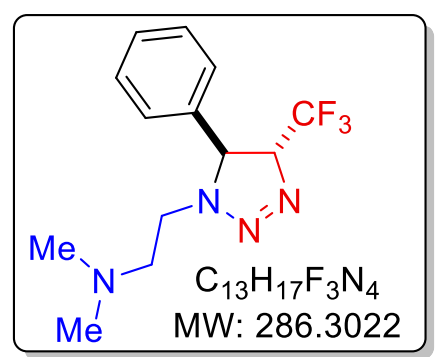

Following the general procedure, treatment of benzaldehyde 1a (53 mg, $0.50 \mathrm{mmol}$ ), N1,N1-dimethylethane-1,2-diamine 2n (53 $\mathrm{mg}, 0.60 \mathrm{mmol}$ ) with trifluorodiazoethane stock solution 3 in toluene $\left(1.83 \mathrm{~mL}, 1.50 \mathrm{mmol}\right.$ ) in the presence of $\mathrm{Ag}_{2} \mathrm{CO}_{3}(4 \mathrm{mg}$, $0.015 \mathrm{mmol}$ ) at $25^{\circ} \mathrm{C}$ for $10 \mathrm{~h}$ followed by column chromatography afforded the product 4ap (96 mg, 67\%). Major isomer: colorless liquid, $\mathbf{R}_{\boldsymbol{f}}$ (Ethyl acetate/Hexane: $\left.30 / 70\right)=0.25 .{ }^{13} \mathrm{C} \mathrm{NMR}(100 \mathrm{MHz}$, $\delta$ ppm/ $\mathrm{CDCl}_{3}$ ): $137.8(\mathrm{C}), 129.5(\mathrm{CH}), 129.5(\mathrm{CH}), 129.0(\mathrm{CH}), 127.2(\mathrm{CH}), 127.2(\mathrm{CH}), 123.8(\mathrm{q}$, $\left.J_{C-F}=276.8 \mathrm{~Hz}, \mathrm{C}\right), 85.2\left(\mathrm{q}, J_{C-F}=28.2 \mathrm{~Hz}, \mathrm{CH}\right), 62.2(\mathrm{CH}), 57.6\left(\mathrm{CH}_{2}\right), 45.6\left(\mathrm{CH}_{2}\right), 45.3\left(\mathrm{CH}_{3}\right), 45.3$ $\left(\mathrm{CH}_{3}\right) .{ }^{1} \mathrm{H}$ NMR $\left(400 \mathrm{MHz}, \delta \mathrm{ppm} / \mathrm{CDCl}_{3}\right): 7.41-7.33(\mathrm{~m}, 3 \mathrm{H}), 7.22-7.20(\mathrm{~m}, 2 \mathrm{H}), 4.78(\mathrm{~d}, J=10.4$ $\mathrm{Hz}, 1 \mathrm{H}), 4.75-4.67(\mathrm{~m}, 1 \mathrm{H}), 3.89-3.82(\mathrm{~m}, 1 \mathrm{H}), 3.45-3.38(\mathrm{~m}, 1 \mathrm{H}), 2.61-2.55(\mathrm{~m}, 1 \mathrm{H}), 2.49-2.44$ $(\mathrm{m}, 1 \mathrm{H}), 2.18(\mathrm{~s}, 6 \mathrm{H}) .{ }^{19} \mathrm{~F} \mathrm{NMR}\left(376 \mathrm{MHz}, \delta \mathrm{ppm} / \mathrm{CDCl}_{3}\right):-73.3(\mathrm{~s})$. HRMS for $\mathrm{C}_{13} \mathrm{H}_{18} \mathrm{~F}_{3} \mathrm{~N}_{4}{ }^{+}$: calcd. $[\mathrm{M}+\mathrm{H}]^{+}: 287.1478$, found: 287.1482 .

Compound 4aq: 4-(2-(5-phenyl-4-(trifluoromethyl)-4,5-dihydro-1H-1,2,3-triazol-1yl)ethyl)morpholine

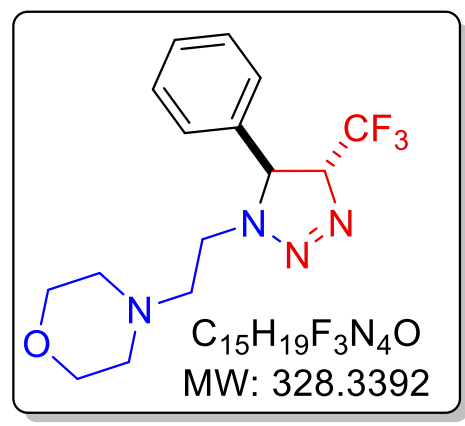

Following the general procedure, treatment of benzaldehyde 1a (53 mg, $0.50 \mathrm{mmol}$ ), 2-morpholinoethan-1-amine 20 (78 mg, $0.60 \mathrm{mmol}$ ) with trifluorodiazoethane stock solution 3 in toluene $(1.83 \mathrm{~mL}, 1.50 \mathrm{mmol})$ in the presence of $\mathrm{Ag}_{2} \mathrm{CO}_{3}(4 \mathrm{mg}$, $0.015 \mathrm{mmol}$ ) at $25{ }^{\circ} \mathrm{C}$ for $8 \mathrm{~h}$ followed by column chromatography afforded the product $4 \mathrm{aq}(120 \mathrm{mg}, 73 \%$ ). Major isomer: colorless liquid, $\mathbf{R}_{\boldsymbol{f}}$ (Ethyl acetate/Hexane: $25 / 75$ ) $=0.25 .{ }^{13} \mathrm{C}$ NMR $\left(100 \mathrm{MHz}, \delta \mathrm{ppm} / \mathrm{CDCl}_{3}\right): 137.8(\mathrm{C}), 129.5(\mathrm{CH})$, $129.5(\mathrm{CH}), 129.1(\mathrm{CH}), 127.1(\mathrm{CH}), 127.1(\mathrm{CH}), 123.9$ (q, $\left.J_{C-F}=276.8 \mathrm{~Hz}, \mathrm{C}\right), 85.3\left(\mathrm{q}, J_{C-F}=28.2\right.$ $\mathrm{Hz}, \mathrm{CH}), 67.1\left(\mathrm{CH}_{2}\right), 67.1\left(\mathrm{CH}_{2}\right), 62.0(\mathrm{~d}, \mathrm{~J}=1.8 \mathrm{~Hz}, \mathrm{CH}), 56.7\left(\mathrm{CH}_{2}\right), 53.5\left(\mathrm{CH}_{2}\right), 53.5\left(\mathrm{CH}_{2}\right), 44.3$ $\left(\mathrm{CH}_{2}\right) .{ }^{1} \mathrm{H}$ NMR $\left(400 \mathrm{MHz}, \delta \mathrm{ppm} / \mathrm{CDCl}_{3}\right): 7.42-7.34(\mathrm{~m}, 3 \mathrm{H}), 7.22-7.20(\mathrm{~m}, 2 \mathrm{H}), 4.86(\mathrm{~d}, J=2.6$ $\mathrm{Hz}, 1 \mathrm{H}), 4.74-4.66(\mathrm{~m}, 1 \mathrm{H}), 4.00-3.93(\mathrm{~m}, 1 \mathrm{H}), 3.65(\mathrm{t}, J=4.4 \mathrm{~Hz}, 4 \mathrm{H}), 3.44-3.38(\mathrm{~m}, 1 \mathrm{H}), 2.59-$ $2.53(\mathrm{~m}, 2 \mathrm{H}), 2.24-2.33(\mathrm{~m}, 4 \mathrm{H}) .{ }^{19} \mathrm{~F}$ NMR $\left(376 \mathrm{MHz}, \delta \mathrm{ppm} / \mathrm{CDCl}_{3}\right):-73.1$ (s). HRMS for $\mathrm{C}_{15} \mathrm{H}_{20} \mathrm{~F}_{3} \mathrm{~N}_{4} \mathrm{O}^{+}$: calcd. [M+H] $]^{+}: 329.1584$, found: 329.1590 . 
Compound 4ar: 7-chloro-N-(3-(5-phenyl-4-(trifluoromethyl)-4,5-dihydro-1H-1,2,3-triazol-1yl)propyl)quinolin-4-amine

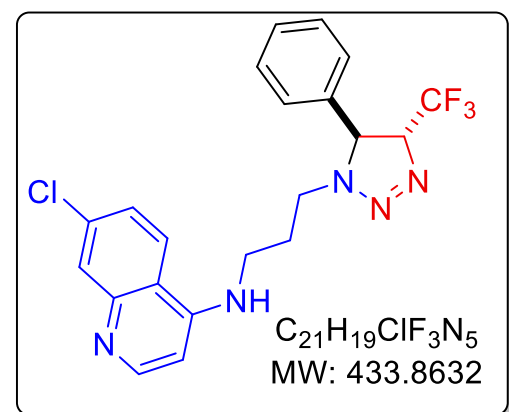

${ }^{13} \mathrm{C}$ NMR (100 MHz, $\delta$ ppm/CDCl $)$ ): 151.9 (CH), 149.6 (C), 149.1 (C), 137.1 (C), 135.1 (C), 129.7 $(\mathrm{CH}), 129.7(\mathrm{CH}), 129.4(\mathrm{CH}), 128.6(\mathrm{CH}), 127.0(\mathrm{CH}), 127.0(\mathrm{CH}), 125.5(\mathrm{CH}), 123.6\left(\mathrm{q}, J_{C-F}=\right.$ $276.9 \mathrm{~Hz}, \mathrm{C}), 121.3(\mathrm{CH}), 117.3(\mathrm{C}), 99.1(\mathrm{CH}), 85.3\left(\mathrm{q}, J_{C-F}=28.2 \mathrm{~Hz}, \mathrm{CH}\right), 62.5(\mathrm{CH}), 45.7\left(\mathrm{CH}_{2}\right)$, $40.3\left(\mathrm{CH}_{2}\right), 26.7\left(\mathrm{CH}_{2}\right) .{ }^{1} \mathrm{H}$ NMR $\left(400 \mathrm{MHz}, \delta \mathrm{ppm} / \mathrm{CDCl}_{3}\right): 8.48(\mathrm{~d}, J=4.8 \mathrm{~Hz}, 1 \mathrm{H}), 7.92(\mathrm{~d}, J=$ $1.2 \mathrm{~Hz}, 1 \mathrm{H}), 7.57(\mathrm{~d}, J=8.8 \mathrm{~Hz}, 1 \mathrm{H}), 7.30(\mathrm{~d}, J=8.8 \mathrm{~Hz}, 1 \mathrm{H}), 7.18-7.16(\mathrm{~m}, 2 \mathrm{H}), 6.33(\mathrm{~d}, J=4.4$ $\mathrm{Hz}, 1 \mathrm{H}), 5.34(\mathrm{~s}, 1 \mathrm{H}), 4.80-4.74(\mathrm{~m}, 1 \mathrm{H}), 4.47(\mathrm{~d}, J=14.4 \mathrm{~Hz}, 1 \mathrm{H}), 3.72-3.65(\mathrm{~m}, 1 \mathrm{H}), 3.54-3.40$ $(\mathrm{m}, 3 \mathrm{H}), 2.14-2.01(\mathrm{~m}, 2 \mathrm{H}) .{ }^{19} \mathrm{~F}$ NMR $\left(376 \mathrm{MHz}, \delta \mathrm{ppm} / \mathrm{CDCl}_{3}\right)$ : -73.1 (s). HRMS for $\mathrm{C}_{21} \mathrm{H}_{20} \mathrm{ClF}_{3} \mathrm{~N}_{5}{ }^{+}$: calcd. [M+H] $]^{+}: 434.1354$, found: 434.1353.

\section{Compound 5a: 5-([1,1'-biphenyl]-4-yl)-1-butyl-4-(trifluoromethyl)-1H-1,2,3-triazole}

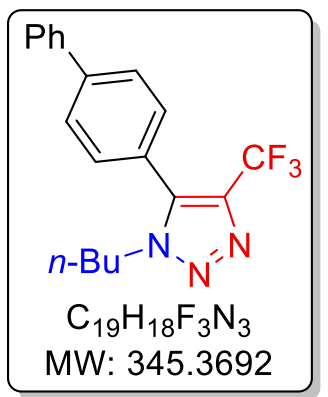

Following the general procedure, treatment of $\mathbf{4 g}(104 \mathrm{mg}, 0.30 \mathrm{mmol})$ with activated $\mathrm{MnO}_{2}(130 \mathrm{mg}, 1.50 \mathrm{mmol})$ in benzene $(5.0 \mathrm{~mL})$ at $80^{\circ} \mathrm{C}$ for $14 \mathrm{~h}$ followed by column chromatography afforded the product $\mathbf{5 a}$ as a brownish liquid (98 mg, 95\%). $\mathbf{R}_{\boldsymbol{f}}$ (Ethyl acetate/Hexane: 10/90) $=0.45$. ${ }^{13} \mathrm{C}$ NMR (100 MHz, $\left.\delta \mathrm{ppm} / \mathrm{CDCl}_{3}\right): 143.4$ (C), 139.7 (C), 137.0 (C), 136.2 $\left(q, J_{C-F}=37.6 \mathrm{~Hz}, \mathrm{C}\right), 130.0(\mathrm{CH}), 130.0(\mathrm{CH}), 129.1(\mathrm{CH}), 129.1(\mathrm{CH}), 128.3$ $(\mathrm{CH}), 127.8(\mathrm{CH}), 127.8(\mathrm{CH}), 127.3(\mathrm{CH}), 127.3(\mathrm{CH}), 123.7(\mathrm{C}), 121.1(\mathrm{q}$, $\left.J_{C-F}=266.7 \mathrm{~Hz}, \mathrm{C}\right), 48.5\left(\mathrm{CH}_{2}\right), 32.1\left(\mathrm{CH}_{2}\right), 19.7\left(\mathrm{CH}_{2}\right), 13.4\left(\mathrm{CH}_{3}\right) .{ }^{1} \mathbf{H}$ NMR $(400 \mathrm{MHz}, \delta$ $\left.\mathrm{ppm} / \mathrm{CDCl}_{3}\right)$ : 7.77-7.74 (m, 2H), 7.67-7.64 (m, 2H), 7.51-7.47 (m, 2H), 7.43-7.39 (m, 3H), 4.27 $(\mathrm{t}, J=7.2 \mathrm{~Hz}, 2 \mathrm{H}), 1.86-1.78(\mathrm{~m}, 2 \mathrm{H}), 1.33-1.24(\mathrm{~m}, 2 \mathrm{H}), 0.87(\mathrm{t}, J=8.0 \mathrm{~Hz}, 3 \mathrm{H}) .{ }^{19} \mathrm{~F}$ NMR $(376$ $\left.\mathrm{MHz}, \delta \mathrm{ppm} / \mathrm{CDCl}_{3}\right):-59.2\left(\mathrm{~d}, J=1.9 \mathrm{~Hz}\right.$ ). HRMS for $\mathrm{C}_{19} \mathrm{H}_{19} \mathrm{~F}_{3} \mathrm{~N}_{3}{ }^{+}$: calcd. $[\mathrm{M}+\mathrm{H}]^{+}:$346.1526, found: 346.1523 . 


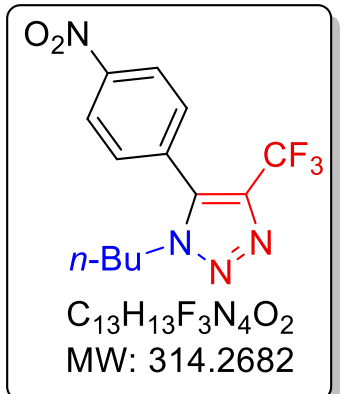

Following the general procedure, treatment of $\mathbf{4 k}(95 \mathrm{mg}, 0.30 \mathrm{mmol})$ with activated $\mathrm{MnO}_{2}(130 \mathrm{mg}, 1.50 \mathrm{mmol})$ in benzene $(5.0 \mathrm{~mL})$ at $80^{\circ} \mathrm{C}$ for $14 \mathrm{~h}$ followed by column chromatography afforded the product $\mathbf{5 b}$ as a colorless liquid ( $89 \mathrm{mg}, 94 \%)$. $\mathbf{R}_{\boldsymbol{f}}$ (Ethyl acetate/Hexane: $\left.15 / 85\right)=$ 0.22. ${ }^{13} \mathrm{C}$ NMR (100 MHz, $\delta \mathrm{ppm} / \mathrm{CDCl}_{3}$ ): 149.2 (C), 136.7 (q, $J_{C-F}=38.1$ $\mathrm{Hz}, \mathrm{C}), 135.0(\mathrm{C}), 131.5(\mathrm{C}), 131.0(\mathrm{CH}), 131.0(\mathrm{CH}), 124.4(\mathrm{CH}), 124.4$ $(\mathrm{CH}), 120.6\left(\mathrm{q}, J_{\mathrm{C}-\mathrm{F}}=267.0 \mathrm{~Hz}, \mathrm{C}\right), 48.9\left(\mathrm{CH}_{2}\right), 32.0\left(\mathrm{CH}_{2}\right), 19.7\left(\mathrm{CH}_{2}\right), 13.4$ $\left(\mathrm{CH}_{3}\right) .{ }^{1} \mathrm{H}$ NMR $\left(400 \mathrm{MHz}, \delta \mathrm{ppm} / \mathrm{CDCl}_{3}\right): 8.42(\mathrm{~d}, J=8.8 \mathrm{~Hz}, 2 \mathrm{H}), 7.58(\mathrm{dd}, J=8.8 \mathrm{~Hz}, J=2.0 \mathrm{~Hz}$, $2 \mathrm{H}), 4.24(\mathrm{t}, J=7.2 \mathrm{~Hz}, 2 \mathrm{H}), 1.83-1.75(\mathrm{~m}, 2 \mathrm{H}), 1.31-1.21(\mathrm{~m}, 2 \mathrm{H}), 0.86(\mathrm{t}, J=7.2 \mathrm{~Hz}, 3 \mathrm{H}) .{ }^{19} \mathrm{~F}$ NMR (376 MHz, $\delta \mathrm{ppm} / \mathrm{CDCl}_{3}$ ): -59.3 (s). HRMS for $\mathrm{C}_{13} \mathrm{H}_{14} \mathrm{~F}_{3} \mathrm{~N}_{4} \mathrm{O}_{2}{ }^{+}$: calcd. [M+H] $]^{+}: 315.1063$, found: 315.1061.

\section{Compound 5c: 5-(benzo[d][1,3]dioxol-5-yl)-1-butyl-4-(trifluoromethyl)-1H-1,2,3-triazole}

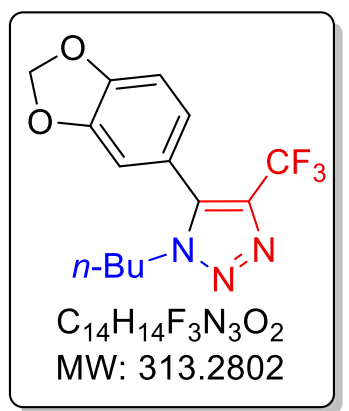

Following the general procedure, treatment of $4 \mathrm{r}(94 \mathrm{mg}, 0.30 \mathrm{mmol})$ with activated $\mathrm{MnO}_{2}(130 \mathrm{mg}, 1.50 \mathrm{mmol})$ in benzene $(5.0 \mathrm{~mL})$ at $80^{\circ} \mathrm{C}$ for $14 \mathrm{~h}$ followed by column chromatography afforded the product $\mathbf{5 c}$ as a colorless liquid (89 $\mathrm{mg}, 95 \%)$. $\mathbf{R}_{\boldsymbol{f}}$ (Ethyl acetate/Hexane: 10/90) $=$ 0.30. ${ }^{13} \mathrm{C}$ NMR (100 MHz, $\left.\delta \mathrm{ppm} / \mathrm{CDCl}_{3}\right): 149.6$ (C), 148.4 (C), 136.9 (C), $136.1\left(\mathrm{~d}, J_{C-F}=37.5 \mathrm{~Hz}, \mathrm{C}\right), 124.0(\mathrm{CH}), 121.0\left(\mathrm{q}, J_{C-F}=266.8 \mathrm{~Hz}, \mathrm{C}\right), 117.9$ (C), $109.7(\mathrm{CH}), 109.1(\mathrm{CH}), 102.0\left(\mathrm{CH}_{2}\right), 48.4\left(\mathrm{CH}_{2}\right), 32.0\left(\mathrm{CH}_{2}\right), 19.7$ $\left(\mathrm{CH}_{2}\right), 13.5\left(\mathrm{CH}_{3}\right) .{ }^{1} \mathrm{H}$ NMR $\left(400 \mathrm{MHz}, \delta \mathrm{ppm} / \mathrm{CDCl}_{3}\right): 6.94(\mathrm{~d}, J=8.0 \mathrm{~Hz}$, $1 \mathrm{H}), 6.79(\mathrm{dd}, J=8.8 \mathrm{~Hz}, 1.6 \mathrm{~Hz}, 1 \mathrm{H}), 6.76(\mathrm{~d}, J=1.6 \mathrm{~Hz}, 1 \mathrm{H}), 6.08(\mathrm{~s}, 2 \mathrm{H}), 4.21(\mathrm{t}, J=7.2 \mathrm{~Hz}$, $2 \mathrm{H}), 1.82-1.75(\mathrm{~m}, 2 \mathrm{H}), 1.31-1.21(\mathrm{~m}, 2 \mathrm{H}), 0.86(\mathrm{t}, J=7.6 \mathrm{~Hz}, 3 \mathrm{H}) .{ }^{19} \mathrm{~F}$ NMR $(376 \mathrm{MHz}, \delta$ ppm/CDCl 3 ): -59.4 (s). HRMS for $\mathrm{C}_{14} \mathrm{H}_{15} \mathrm{~F}_{3} \mathrm{~N}_{3} \mathrm{O}_{2}{ }^{+}$: calcd. $[\mathrm{M}+\mathrm{H}]^{+}: 314.1111$, found: 314.1112 .

\section{Compound 5d: (E)-1-butyl-5-styryl-4-(trifluoromethyl)-1H-1,2,3-triazole}

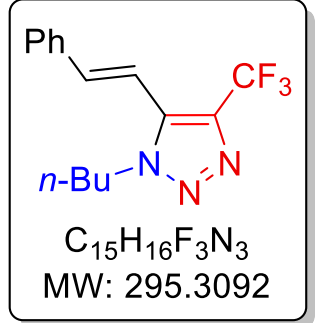

Following the general procedure, treatment of $4 \mathrm{aa}(89 \mathrm{mg}, 0.30 \mathrm{mmol}$ ) with activated $\mathrm{MnO}_{2}(130 \mathrm{mg}, 1.50 \mathrm{mmol})$ in benzene $(5.0 \mathrm{~mL})$ at $80{ }^{\circ} \mathrm{C}$ for $14 \mathrm{~h}$ followed by column chromatography afforded the product $\mathbf{5 d}$ as a yellowish liquid (86 mg, 97\%). $\mathbf{R}_{\boldsymbol{f}}$ (Ethyl acetate/Hexane: 10/90) $=0.40$. ${ }^{13} \mathrm{C}$ NMR (100 MHz, $\left.\delta \mathrm{ppm} / \mathrm{CDCl}_{3}\right): 139.7$ (d, $\left.J_{C-F}=1.7 \mathrm{~Hz}, \mathrm{C}\right), 135.3(\mathrm{CH})$, $135.1\left(q, J_{C-F}=29.7 \mathrm{~Hz}, \mathrm{C}\right), 134.2(\mathrm{C}), 129.9(\mathrm{CH}), 129.2(\mathrm{CH}), 129.2(\mathrm{CH})$, $127.3(\mathrm{CH}), 127.3(\mathrm{CH}), 121.4\left(\mathrm{q}, J_{\mathrm{C}-F}=213.1 \mathrm{~Hz}, \mathrm{C}\right), 109.4(\mathrm{CH}), 48.9\left(\mathrm{CH}_{2}\right), 31.9\left(\mathrm{CH}_{2}\right), 19.8$ $\left(\mathrm{CH}_{2}\right), 13.6\left(\mathrm{CH}_{3}\right) .{ }^{1} \mathrm{H}$ NMR $\left(400 \mathrm{MHz}, \delta \mathrm{ppm} / \mathrm{CDCl}_{3}\right): 7.54-7.51(\mathrm{~m}, 2 \mathrm{H}), 7.45-7.36(\mathrm{~m}, 3 \mathrm{H}), 7.14$ (d, $J=16.8 \mathrm{~Hz}, 1 \mathrm{H}), 6.84(\mathrm{~d}, J=16.8 \mathrm{~Hz}, 1 \mathrm{H}), 4.42(\mathrm{t}, J=7.6 \mathrm{~Hz}, 2 \mathrm{H}), 1.97-1.89(\mathrm{~m}, 2 \mathrm{H}), 1.45-$ $1.36(\mathrm{~m}, 2 \mathrm{H}), 0.98(\mathrm{t}, J=7.6 \mathrm{~Hz}, 3 \mathrm{H}) .{ }^{19} \mathrm{~F} \mathrm{NMR}\left(376 \mathrm{MHz}, \delta \mathrm{ppm} / \mathrm{CDCl}_{3}\right):-59.9$ (s). HRMS for $\mathrm{C}_{15} \mathrm{H}_{17} \mathrm{~F}_{3} \mathrm{~N}_{3}{ }^{+}$: calcd. [M+H] $]^{+}: 296.1369$, found: 296.1366 . 


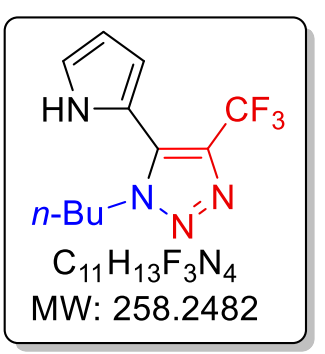

Following the general procedure, treatment of $4 \mathbf{v}(78 \mathrm{mg}, 0.30 \mathrm{mmol})$ with activated $\mathrm{MnO}_{2}(130 \mathrm{mg}, 1.50 \mathrm{mmol})$ in benzene $(5.0 \mathrm{~mL})$ at $80{ }^{\circ} \mathrm{C}$ for $14 \mathrm{~h}$ followed by column chromatography afforded the product $5 \mathrm{e}$ as a reddish liquid (74 $\mathrm{mg}, 95 \%) . \mathbf{R}_{f}$ (Ethyl acetate/Hexane: $\left.15 / 85\right)=0.23 .{ }^{13} \mathbf{C}$ NMR $\left(100 \mathrm{MHz}, \delta \mathrm{ppm} / \mathrm{CDCl}_{3}\right): 135.0\left(q, J_{C-F}=37.7 \mathrm{~Hz}, \mathrm{C}\right), 130.5(\mathrm{C}), 122.0$ $(\mathrm{CH}), 121.2\left(\mathrm{q}, J_{\mathrm{C}-\mathrm{F}}=266.4 \mathrm{~Hz}, \mathrm{C}\right), 113.7(\mathrm{C}), 112.6(\mathrm{CH}), 110.4(\mathrm{CH}), 48.9$ $\left(\mathrm{CH}_{2}\right), 32.0\left(\mathrm{CH}_{2}\right), 19.8\left(\mathrm{CH}_{2}\right), 13.5\left(\mathrm{CH}_{3}\right) .{ }^{1} \mathrm{H} \mathrm{NMR}\left(400 \mathrm{MHz}, \delta \mathrm{ppm} / \mathrm{CDCl}_{3}\right): 8.93(\mathrm{~s}, 1 \mathrm{H}), 7.06-$ $7.04(\mathrm{~m}, 1 \mathrm{H}), 6.49-6.47(\mathrm{~m}, 1 \mathrm{H}), 6.40-6.38(\mathrm{~m}, 1 \mathrm{H}), 4.36(\mathrm{t}, J=7.6 \mathrm{~Hz}, 2 \mathrm{H}), 1.88-1.80(\mathrm{~m}, 2 \mathrm{H})$, 1.38-1.29 (m, 2H), $0.90(\mathrm{t}, J=7.2 \mathrm{~Hz}, 3 \mathrm{H}) .{ }^{19} \mathrm{~F}$ NMR (376 MHz, $\left.\delta \mathrm{ppm} / \mathrm{CDCl}_{3}\right):-59.3(\mathrm{~s})$. HRMS for $\mathrm{C}_{11} \mathrm{H}_{14} \mathrm{~F}_{3} \mathrm{~N}_{4}^{+}$: calcd. [M+H] $]^{+}: 259.1165$, found: 259.1163 .

\section{Compound 5f: 1-butyl-5-(furan-2-yl)-4-(trifluoromethyl)-1H-1,2,3-triazole}

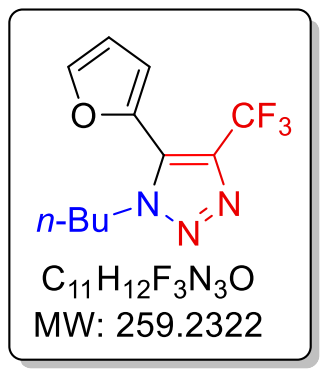

Following the general procedure, treatment of $4 \mathbf{w}(78 \mathrm{mg}, 0.30 \mathrm{mmol})$ with activated $\mathrm{MnO}_{2}(130 \mathrm{mg}, 1.50 \mathrm{mmol})$ in benzene $(5.0 \mathrm{~mL})$ at $80^{\circ} \mathrm{C}$ for $14 \mathrm{~h}$ followed by column chromatography afforded the product $\mathbf{5} \mathbf{f}$ as a reddish liquid (75 $\mathrm{mg}, 97 \%) . \mathbf{R}_{f}$ (Ethyl acetate/Hexane: 10/90) $=0.33$. ${ }^{13} \mathrm{C}$ NMR $\left(100 \mathrm{MHz}, \delta \mathrm{ppm} / \mathrm{CDCl}_{3}\right): 145.1(\mathrm{CH}), 138.3(\mathrm{C}), 135.2\left(\mathrm{q}, J_{\mathrm{C}-F}=\right.$ $38.2 \mathrm{~Hz}, \mathrm{C}), 127.8\left(\mathrm{~d}, J_{C-F}=1.7 \mathrm{~Hz}, \mathrm{C}\right), 120.9\left(\mathrm{q}, J_{C-F}=266.7 \mathrm{~Hz}, \mathrm{C}\right), 114.4$ (q, $\left.J_{C-F}=2.3 \mathrm{~Hz}, \mathrm{CH}\right), 112.2(\mathrm{CH}), 50.2\left(\mathrm{CH}_{2}\right), 32.0\left(\mathrm{CH}_{2}\right), 19.7\left(\mathrm{CH}_{2}\right), 13.5$

$\left(\mathrm{CH}_{3}\right) .{ }^{1} \mathrm{H}$ NMR $\left(400 \mathrm{MHz}, \delta \mathrm{ppm} / \mathrm{CDCl}_{3}\right): 7.65(\mathrm{~d}, J=1.6 \mathrm{~Hz}, 1 \mathrm{H}), 6.84(\mathrm{~d}, J=3.2 \mathrm{~Hz}, 1 \mathrm{H}), 6.61-$ $6.60(\mathrm{~m}, 1 \mathrm{H}), 4.53(\mathrm{t}, J=7.2 \mathrm{~Hz}, 2 \mathrm{H}), 1.88-1.80(\mathrm{~m}, 2 \mathrm{H}), 1.39-1.30(\mathrm{~m}, 2 \mathrm{H}), 0.91(\mathrm{t}, J=7.2 \mathrm{~Hz}$, 3H). ${ }^{19} \mathrm{~F}$ NMR (376 MHz, $\delta \mathrm{ppm} / \mathrm{CDCl}_{3}$ ): -60.6 (s). HRMS for $\mathrm{C}_{11} \mathrm{H}_{13} \mathrm{~F}_{3} \mathrm{~N}_{3} \mathrm{O}^{+}$: calcd. $[\mathrm{M}+\mathrm{H}]^{+}$: 260.1005 , found: 260.1006 .

\section{Compound 5g: 1-butyl-5-(1H-imidazol-4-yl)-4-(trifluoromethyl)-1H-1,2,3-triazole}

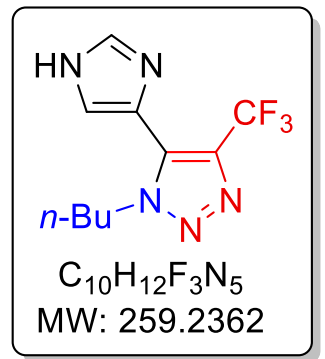

Following the general procedure, treatment of $4 \mathbf{x}(78 \mathrm{mg}, 0.30 \mathrm{mmol})$ with activated $\mathrm{MnO}_{2}(130 \mathrm{mg}, 1.50 \mathrm{mmol})$ in benzene $(5.0 \mathrm{~mL})$ at $80^{\circ} \mathrm{C}$ for $14 \mathrm{~h}$ followed by column chromatography afforded the product $\mathbf{5 g}$ as a white solid (73 mg, 94\%). $\mathbf{R}_{\boldsymbol{f}}$ (Ethyl acetate/Hexane: 40/60) $=0.20$. Mp 105-107 ${ }^{\circ} \mathrm{C} .{ }^{13} \mathrm{C}$ NMR $\left(100 \mathrm{MHz}, \delta \mathrm{ppm} / \mathrm{CDCl}_{3}\right): 136.7(\mathrm{CH}), 134.6\left(\mathrm{q}, J_{\mathrm{C}-\mathrm{F}}=\right.$ $37.7 \mathrm{~Hz}, \mathrm{C}), 131.4(\mathrm{C}), 125.3(\mathrm{C}), 121.3\left(\mathrm{q}, J_{\mathrm{C}-\mathrm{F}}=266.4 \mathrm{~Hz}, \mathrm{C}\right), 118.9(\mathrm{CH})$, $49.9\left(\mathrm{CH}_{2}\right), 32.0\left(\mathrm{CH}_{2}\right), 19.7\left(\mathrm{CH}_{2}\right), 13.5\left(\mathrm{CH}_{3}\right) .{ }^{1} \mathrm{H}$ NMR $(400 \mathrm{MHz}, \delta$ ppm/ $\left.\mathrm{CDCl}_{3}\right): 7.82(\mathrm{~d}, J=1.2 \mathrm{~Hz}, 1 \mathrm{H}), 7.47(\mathrm{~s}, 1 \mathrm{H}), 4.74(\mathrm{t}, J=7.2 \mathrm{~Hz}, 2 \mathrm{H}), 1.90-1.82(\mathrm{~m}, 2 \mathrm{H})$, 1.38-1.29 (m, 2H), $0.90(\mathrm{t}, J=7.6 \mathrm{~Hz}, 3 \mathrm{H}) .{ }^{19} \mathrm{~F}$ NMR (376 MHz, $\left.\delta \mathrm{ppm} / \mathrm{CDCl}_{3}\right):-60.0$ (s). HRMS for $\mathrm{C}_{10} \mathrm{H}_{13} \mathrm{~F}_{3} \mathrm{~N}_{5}^{+}$: calcd. [M+H] $]^{+}: 260.1118$, found: 260.1117 . 


\section{Compound 5h: 1-heptyl-5-phenyl-4-(trifluoromethyl)-1H-1,2,3-triazole}

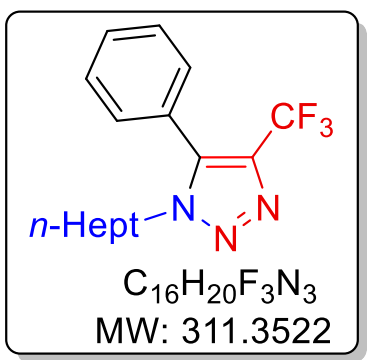

Following the general procedure, treatment of $4 \mathrm{ad}(94 \mathrm{mg}, 0.30$ $\mathrm{mmol})$ with activated $\mathrm{MnO}_{2}(130 \mathrm{mg}, 1.50 \mathrm{mmol})$ in benzene $(5.0 \mathrm{~mL})$ at $80{ }^{\circ} \mathrm{C}$ for $14 \mathrm{~h}$ followed by column chromatography afforded the product $5 \mathrm{~h}$ as a colourless liquid (90 mg, 96\%). $\mathbf{R}_{\boldsymbol{f}}$ (Ethyl acetate/Hexane: $10 / 90)=0.42 .{ }^{13} \mathrm{C}$ NMR $\left(100 \mathrm{MHz}, \delta \mathrm{ppm} / \mathrm{CDCl}_{3}\right)$ : $137.2(\mathrm{C}), 136.2\left(\mathrm{~d}, J_{C-F}=37.7 \mathrm{~Hz}, \mathrm{C}\right), 130.6(\mathrm{CH}), 129.6(\mathrm{CH}), 129.6(\mathrm{CH})$, $129.2(\mathrm{CH}), 129.2(\mathrm{CH}), 125.0(\mathrm{C}), 121.0\left(\mathrm{q}, J_{C-F}=266.8 \mathrm{~Hz}, \mathrm{C}\right), 48.7(\mathrm{CH}-$ 2), $31.5\left(\mathrm{CH}_{2}\right), 30.0\left(\mathrm{CH}_{2}\right), 28.6\left(\mathrm{CH}_{2}\right), 26.4\left(\mathrm{CH}_{2}\right), 22.6\left(\mathrm{CH}_{2}\right), 14.1\left(\mathrm{CH}_{3}\right) .{ }^{1} \mathrm{H} \mathbf{N M R}(400 \mathrm{MHz} \delta$ $\left.\mathrm{ppm} / \mathrm{CDCl}_{3}\right): 7.57-7.51(\mathrm{~m}, 3 \mathrm{H}), 7.34-7.30(\mathrm{~d}, J=5.2 \mathrm{~Hz}, 2 \mathrm{H}), 4.21(\mathrm{t}, J=6.0 \mathrm{~Hz}, 2 \mathrm{H}), 1.79-1.75$ $(\mathrm{m}, 2 \mathrm{H}), 1.25-1.18(\mathrm{~m}, 8 \mathrm{H}), 0.84(\mathrm{t}, J=7.6 \mathrm{~Hz}, 3 \mathrm{H}) .{ }^{19} \mathrm{~F} \mathrm{NMR}\left(376 \mathrm{MHz}, \delta \mathrm{ppm} / \mathrm{CDCl}_{3}\right):-59.3(\mathrm{~s})$. HRMS for $\mathrm{C}_{16} \mathrm{H}_{21} \mathrm{~F}_{3} \mathrm{~N}_{3}{ }^{+}$: calcd. [M+H] $]^{+}: 312.1682$, found: 312.1679 .

\section{Compound 5i: 1-isopropyl-5-phenyl-4-(trifluoromethyl)-1H-1,2,3-triazole}

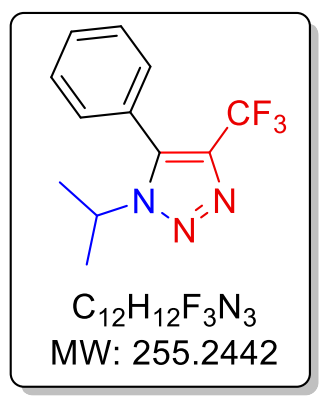

Following the general procedure, treatment of $4 \mathrm{al}(77 \mathrm{mg}, 0.30 \mathrm{mmol})$ with activated $\mathrm{MnO}_{2}(130 \mathrm{mg}, 1.50 \mathrm{mmol})$ in benzene $(5.0 \mathrm{~mL})$ at $80{ }^{\circ} \mathrm{C}$ for $14 \mathrm{~h}$ followed by column chromatography afforded the product $\mathbf{5 i}$ as a white solid (72 mg, 94\%). $\mathbf{R}_{\boldsymbol{f}}$ (Ethyl acetate/Hexane: 10/90) $=0.42 . \mathbf{M p}$ 122-124 ${ }^{\circ} \mathrm{C} .{ }^{13} \mathrm{C}$ NMR $\left(100 \mathrm{MHz}, \delta \mathrm{ppm} / \mathrm{CDCl}_{3}\right): 136.4(\mathrm{C}), 136.0\left(\mathrm{q}, J_{\mathrm{C}-\mathrm{F}}=\right.$ $37.5 \mathrm{~Hz}, \mathrm{C}), 130.6(\mathrm{CH}), 129.7(\mathrm{CH}), 129.7(\mathrm{CH}), 129.2(\mathrm{CH}), 129.2(\mathrm{CH})$, $125.2(\mathrm{C}), 121.8\left(\mathrm{q}, J_{\mathrm{C}-\mathrm{F}}=266.7 \mathrm{~Hz}, \mathrm{C}\right), 51.6(\mathrm{CH}), 23.1\left(\mathrm{CH}_{3}\right), 23.1\left(\mathrm{CH}_{3}\right) .{ }^{1} \mathrm{H}$

NMR (400 MHz, $\left.\delta ~ p p m / \mathrm{CDCl}_{3}\right): 7.57-7.50(\mathrm{~m}, 3 \mathrm{H}), 7.34-7.30(\mathrm{~m}, 2 \mathrm{H}), 4.51-4.40(\mathrm{~m}, 1 \mathrm{H}), 1.57$ (d, $J=6.8 \mathrm{~Hz}, 6 \mathrm{H}) .{ }^{19} \mathrm{~F} \mathrm{NMR}\left(376 \mathrm{MHz}, \delta \mathrm{ppm} / \mathrm{CDCl}_{3}\right)$ : -59.3 (s). HRMS for $\mathrm{C}_{12} \mathrm{H}_{13} \mathrm{~F}_{3} \mathrm{~N}_{3}{ }^{+}$: calcd. $[\mathrm{M}+\mathrm{H}]^{+}:$256.1056, found: 256.1058 . 


\section{X-Ray Data Collection and Structure Refinement Details for compound 4s:}

A good quality colorless single crystal of size $0.18 \times 0.14 \times 0.05 \mathrm{~mm}$, was selected under a polarizing microscope and was mounted on a glass fiber for data collection. Single crystal Xray data for compound $4 \mathrm{~s}$ were collected on the Rigaku Kappa 3 circle diffractometer equipped with the AFC12 goniometer and enhanced sensitivity (HG) Saturn724+ CCD detector in the $4 \times 4$ bin mode using the monochromated Mo-K $\alpha$ radiation generated from the microfocus sealed tube MicroMax-003 X-ray generator equipped with specially designed confocal multilayer optics. Data collection was performed using $\omega$-scans of $0.5^{\circ}$ steps at 293(2) K. Cell determination, data collection and data reduction was performed using the Rigaku CrystalClear-SM Expert 2.1 b24 software. ${ }^{1}$ Structure solution and refinement were performed by using SHELXTL-NT. ${ }^{2}$ Refinement of coordinates and anisotropic thermal parameters of non-hydrogen atoms were carried out by the full-matrix least-squares method. The hydrogen atoms attached to carbon atoms were generated with idealized geometries and isotropically refined using a riding model.

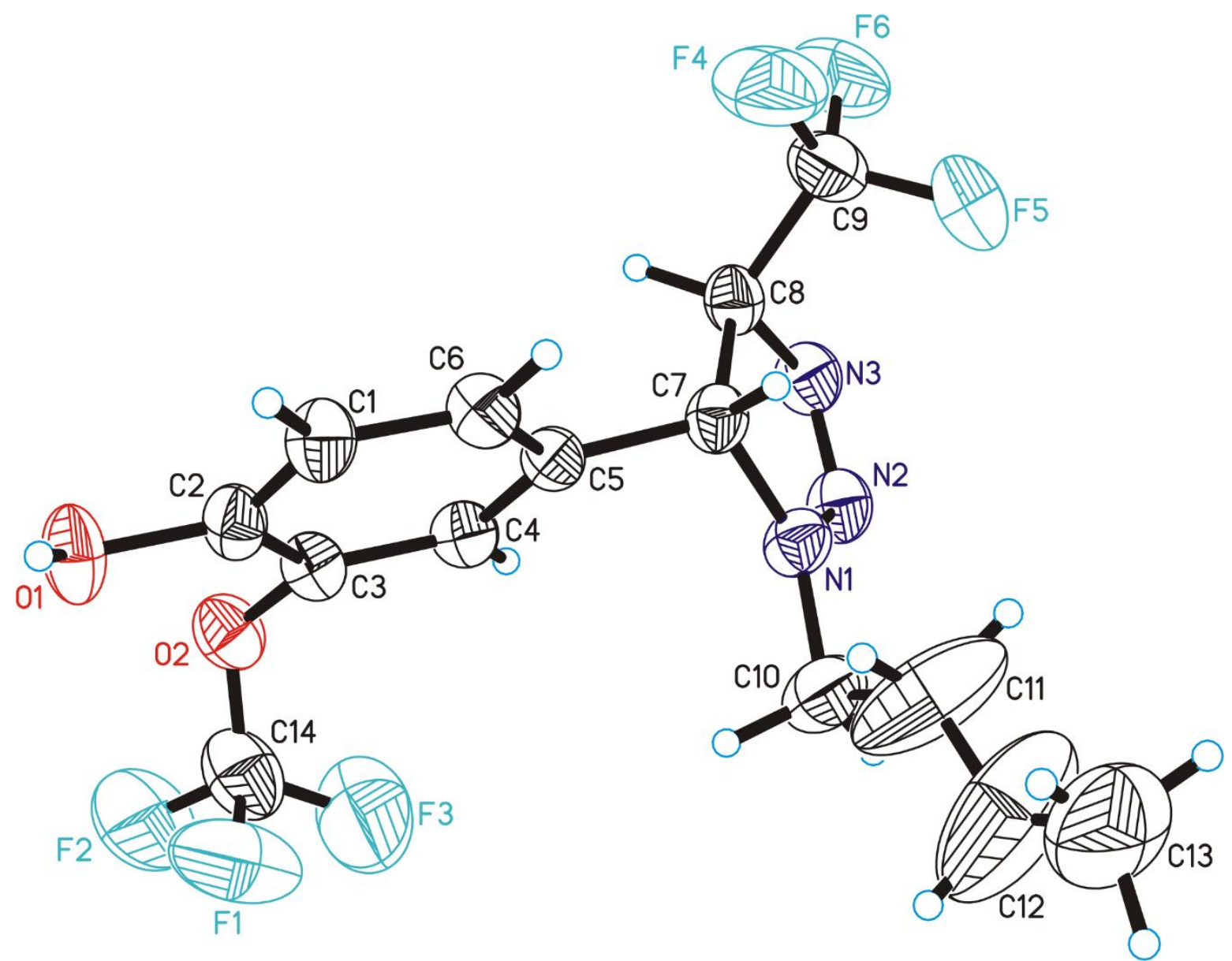

Figure S1. ORTEP diagram drawn with $30 \%$ ellipsoid probability for non-H atoms of the crystal structure of compound $4 \mathrm{~s}$ determined at $293 \mathrm{~K}$. 
Table S1 Crystal data and structure refinement details for $\mathbf{4 s .}$

\begin{tabular}{|c|c|}
\hline Compound & $4 s$ \\
\hline Empirical formula & $\mathrm{C}_{14} \mathrm{H}_{15} \mathrm{~F}_{6} \mathrm{~N}_{3} \mathrm{O}_{2}$ \\
\hline Formula weight & 371.29 \\
\hline Crystal System & Monoclinic \\
\hline Space group & $P 21 / c$ \\
\hline$a(\AA ̊ \cap)$ & $12.226(5)$ \\
\hline$b(\AA)$ & $15.961(5)$ \\
\hline$c(\AA)$ & $9.348(4)$ \\
\hline$\alpha\left(^{\circ}\right)$ & 90.00 \\
\hline$\beta\left(^{\circ}\right)$ & $110.734(5)$ \\
\hline$v\left(^{\circ}\right)$ & 90.00 \\
\hline$V\left(\AA^{3}\right)$ & $1706.0(11)$ \\
\hline Z & 4 \\
\hline$D_{c}\left(g / \mathrm{cm}^{3}\right)$ & 1.446 \\
\hline$F_{000}$ & 760 \\
\hline$\mu\left(\mathrm{mm}^{-1}\right)$ & 0.141 \\
\hline$\theta_{\max }\left({ }^{\circ}\right)$ & 25.39 \\
\hline Total reflections & 10171 \\
\hline Unique reflections & 3025 \\
\hline Reflections $[I>2 \sigma(I)]$ & 1498 \\
\hline Parameters & 229 \\
\hline$R_{\text {int }}$ & 0.0481 \\
\hline Goodness-of-fit & 1.031 \\
\hline$R\left[F^{2}>2 \sigma\left(F^{2}\right)\right]$ & 0.0800 \\
\hline$w R\left(F^{2}\right.$, all data $)$ & 0.2728 \\
\hline CCDC No. & 1859408 \\
\hline
\end{tabular}

1. CrystalClear 2.1, Rigaku Corporation, Tokyo, Japan

2. Sheldrick, G. M. Acta Crystallogr., Sect. A 2008, 64, 112-122. 


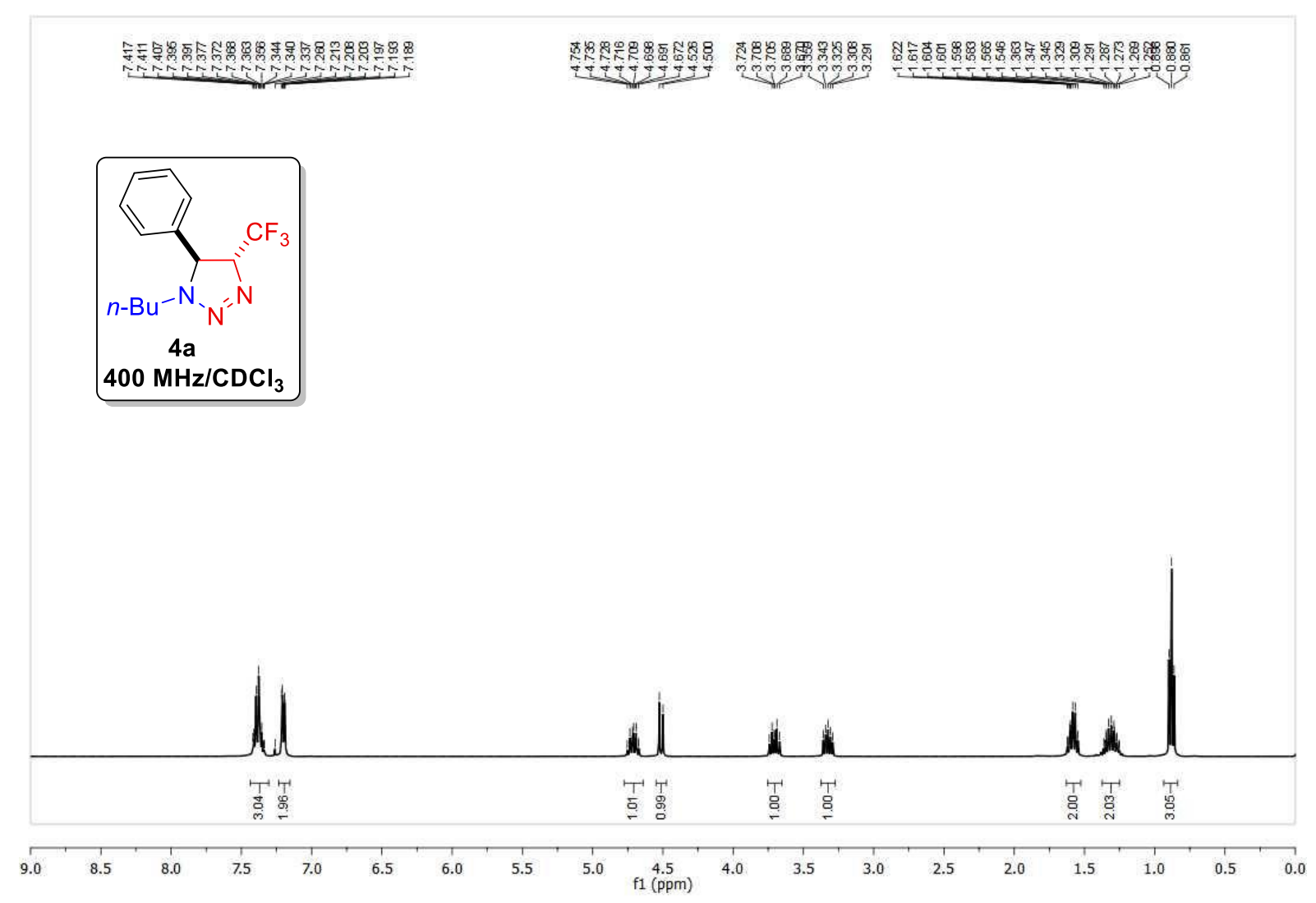

\section{을}

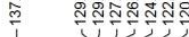

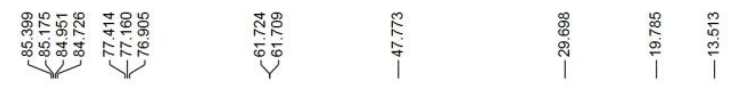
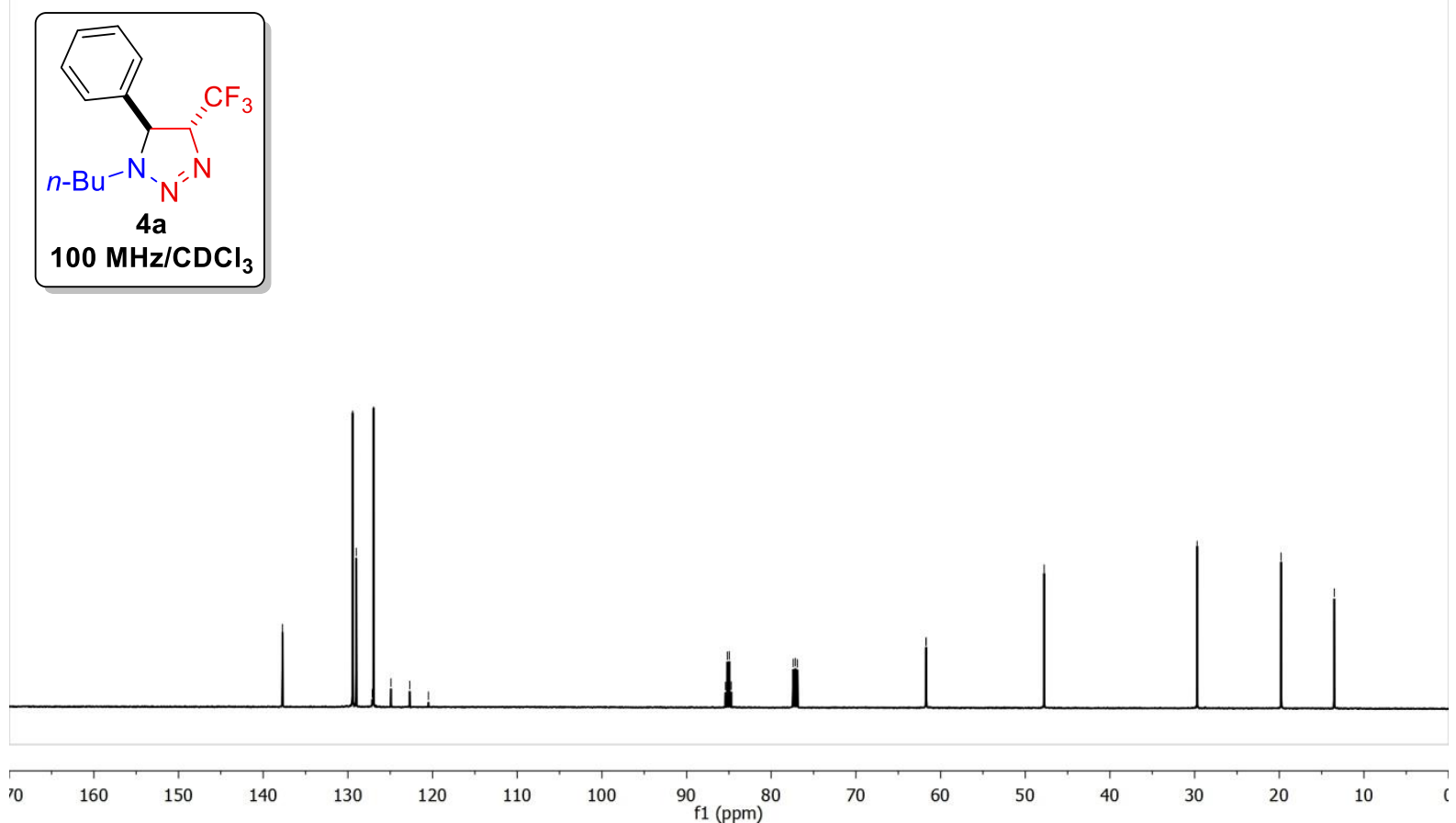


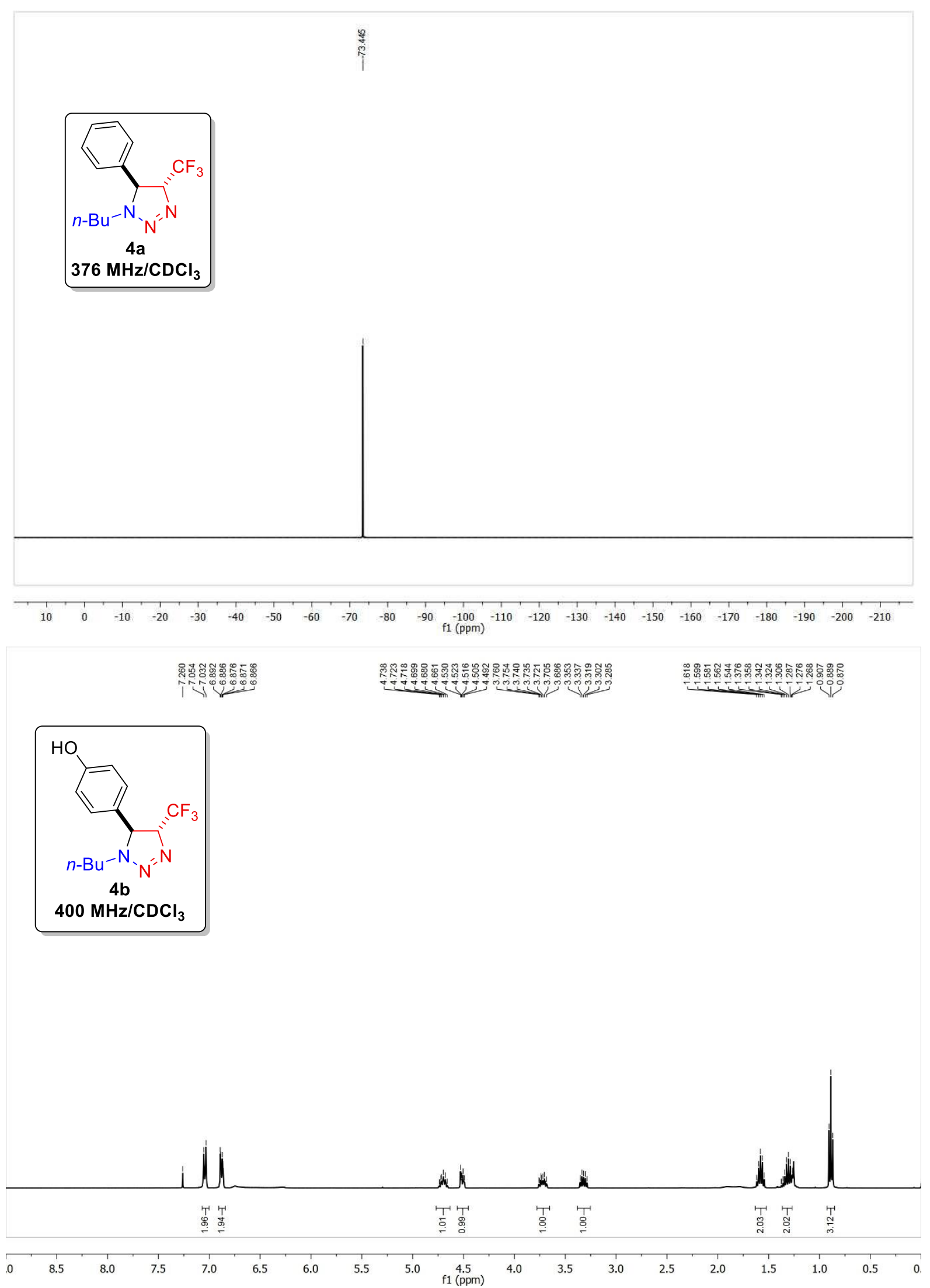



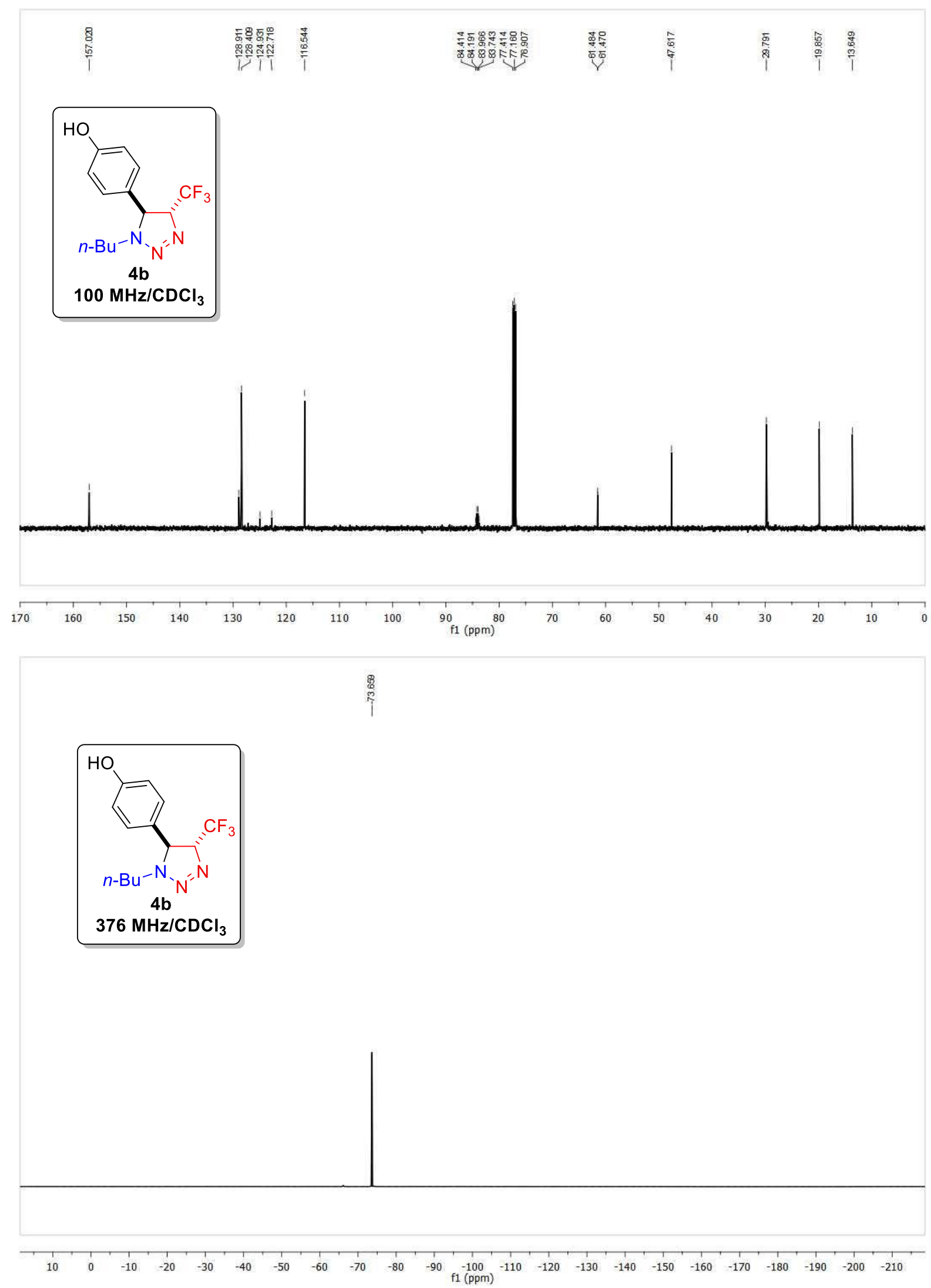

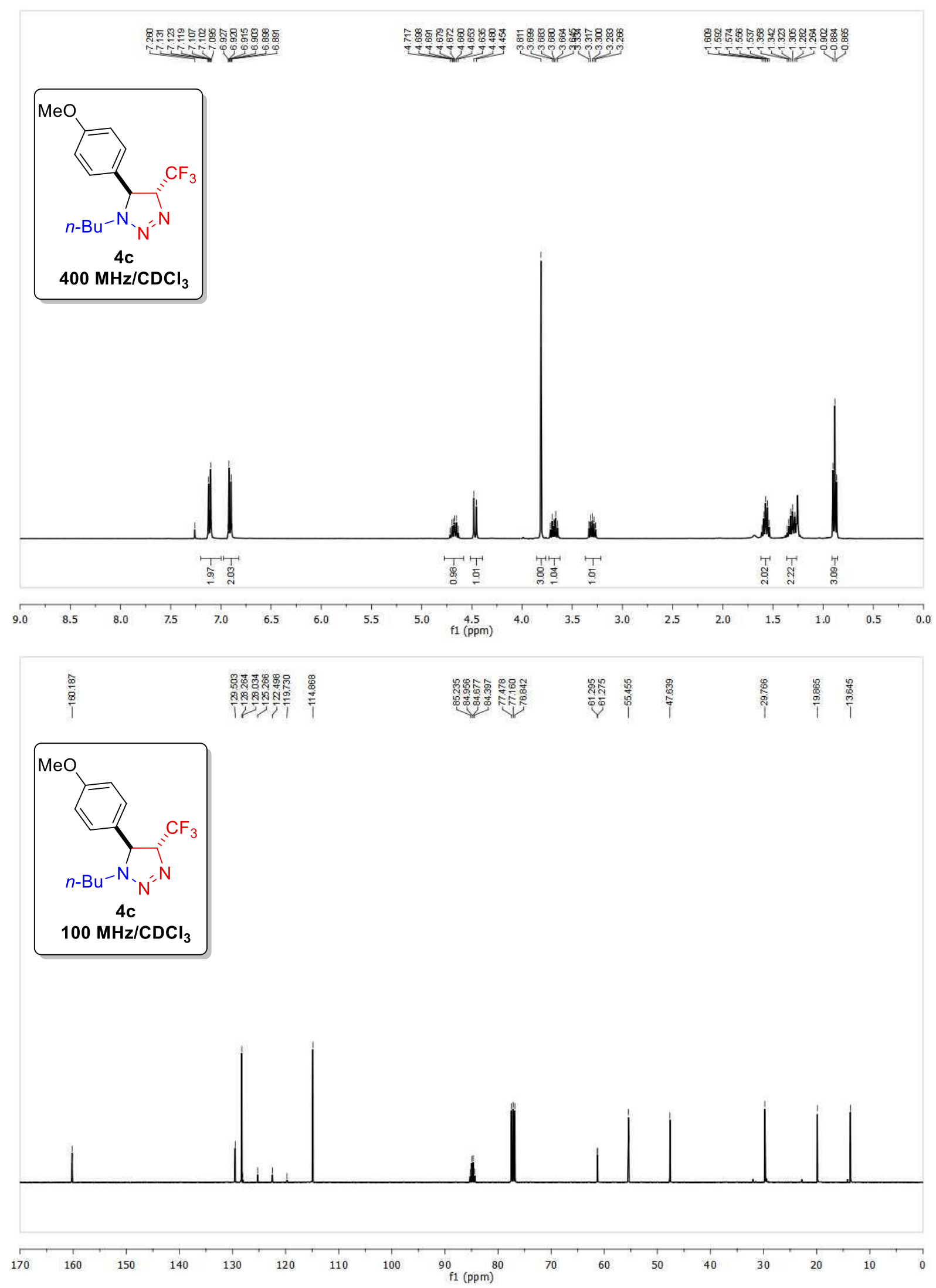


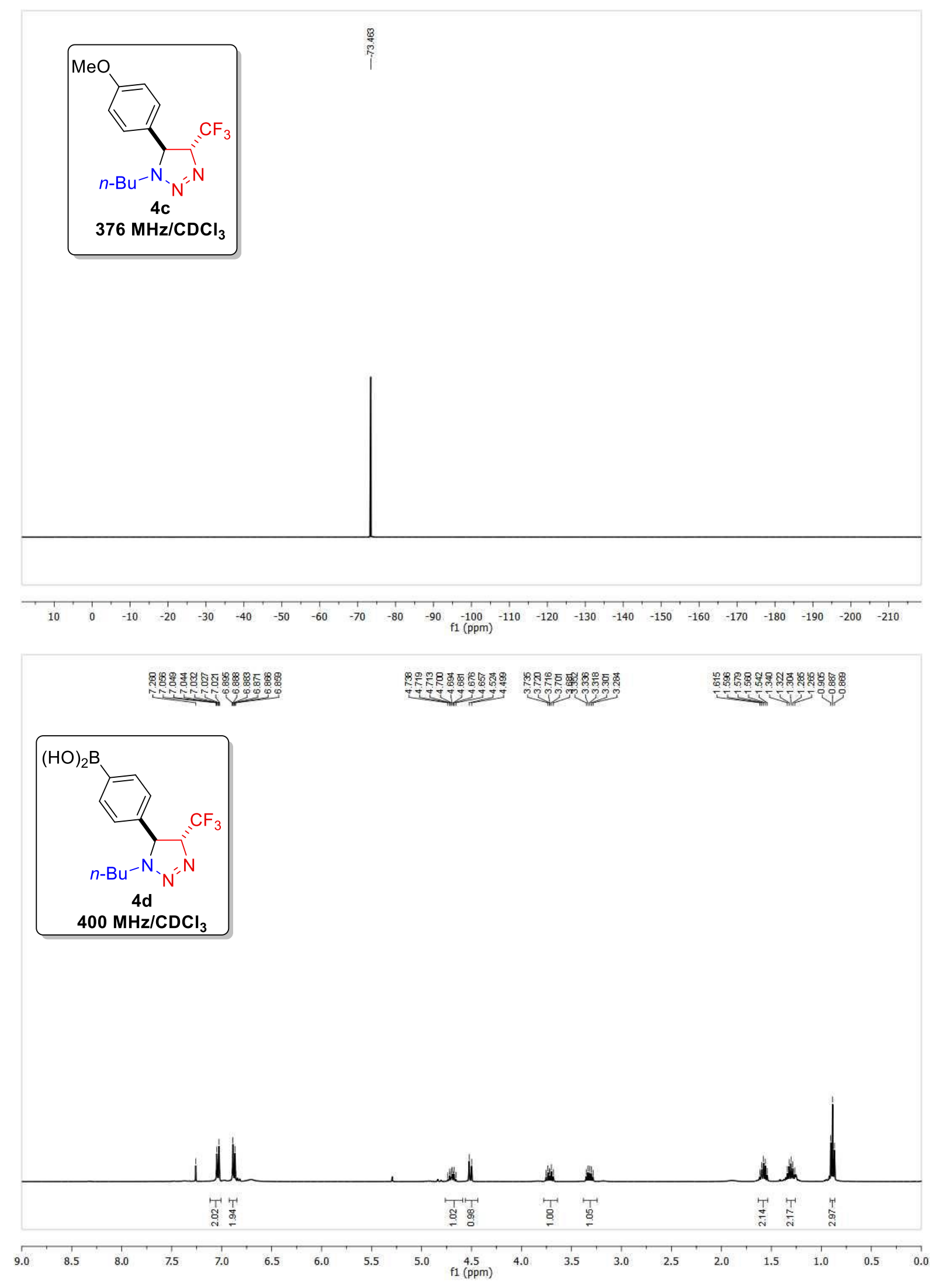



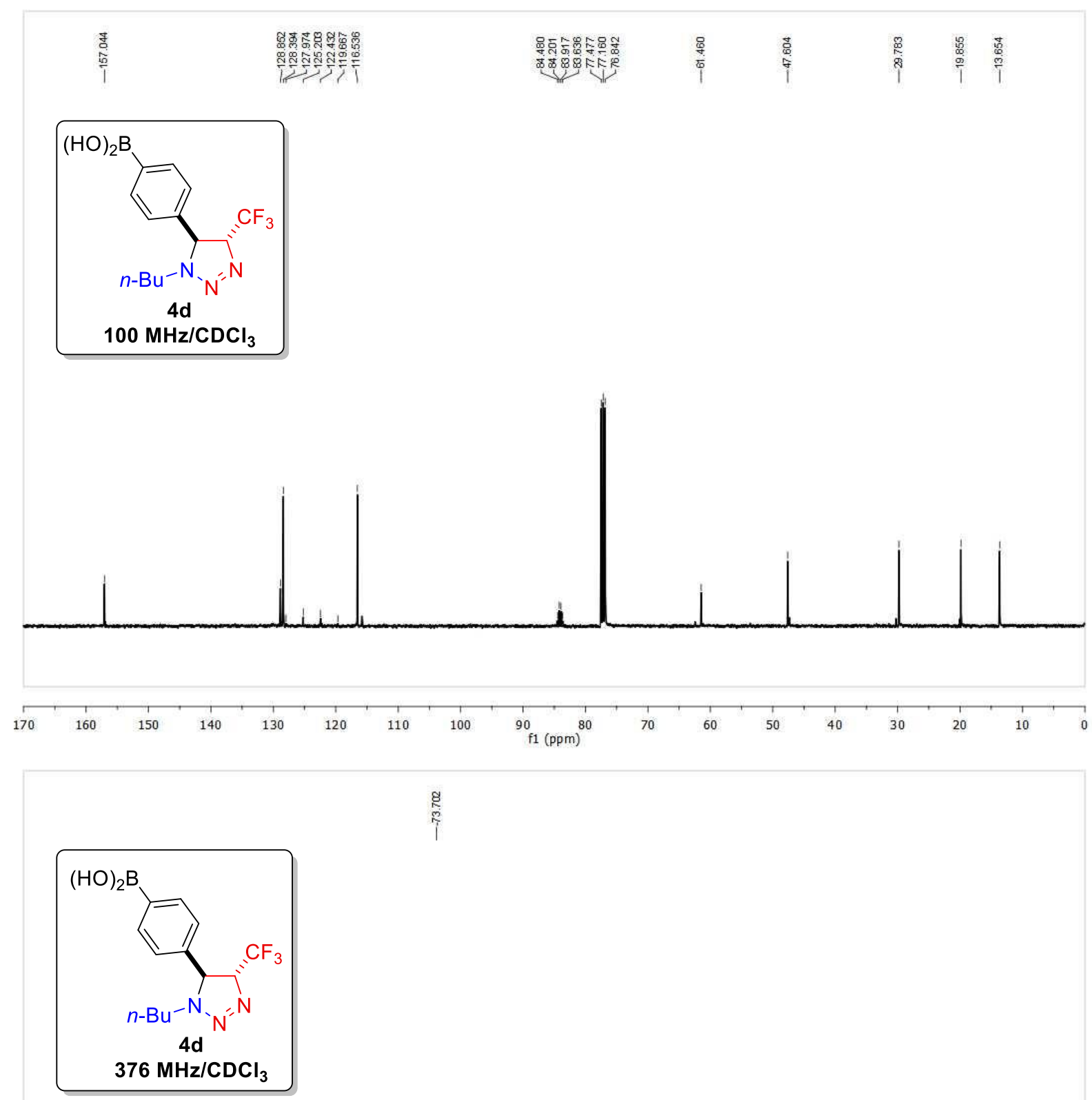

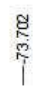

$(\mathrm{HO})_{2} \mathrm{~B}$

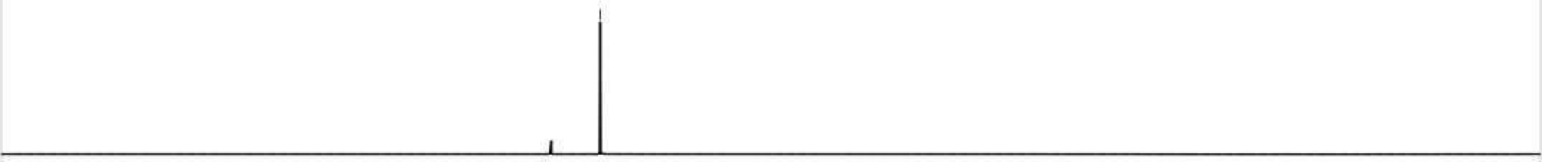

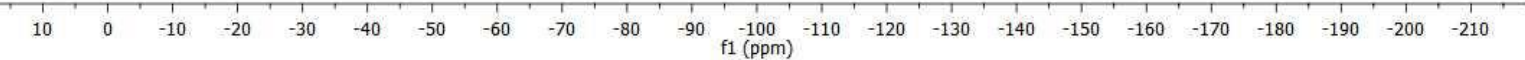



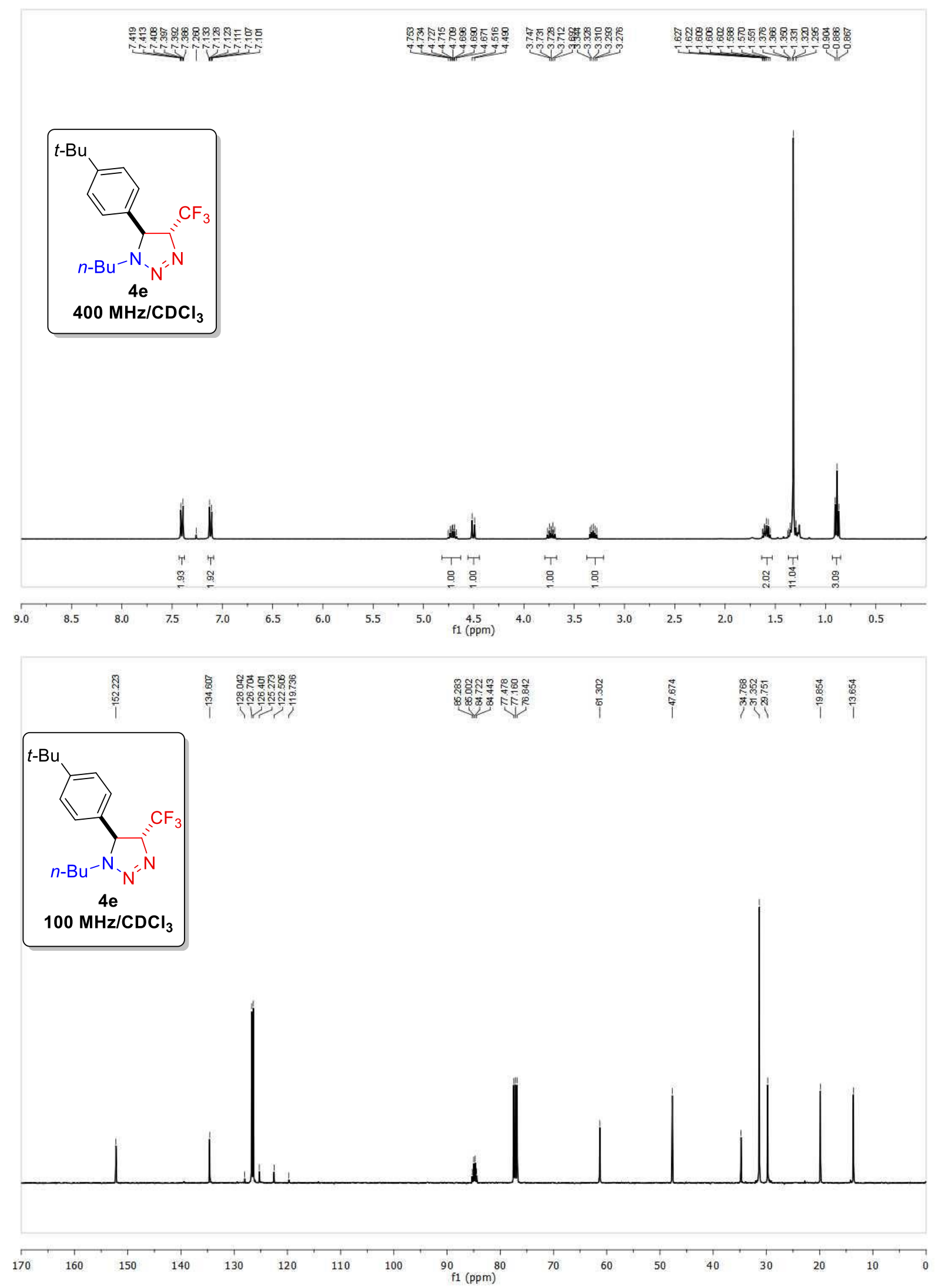


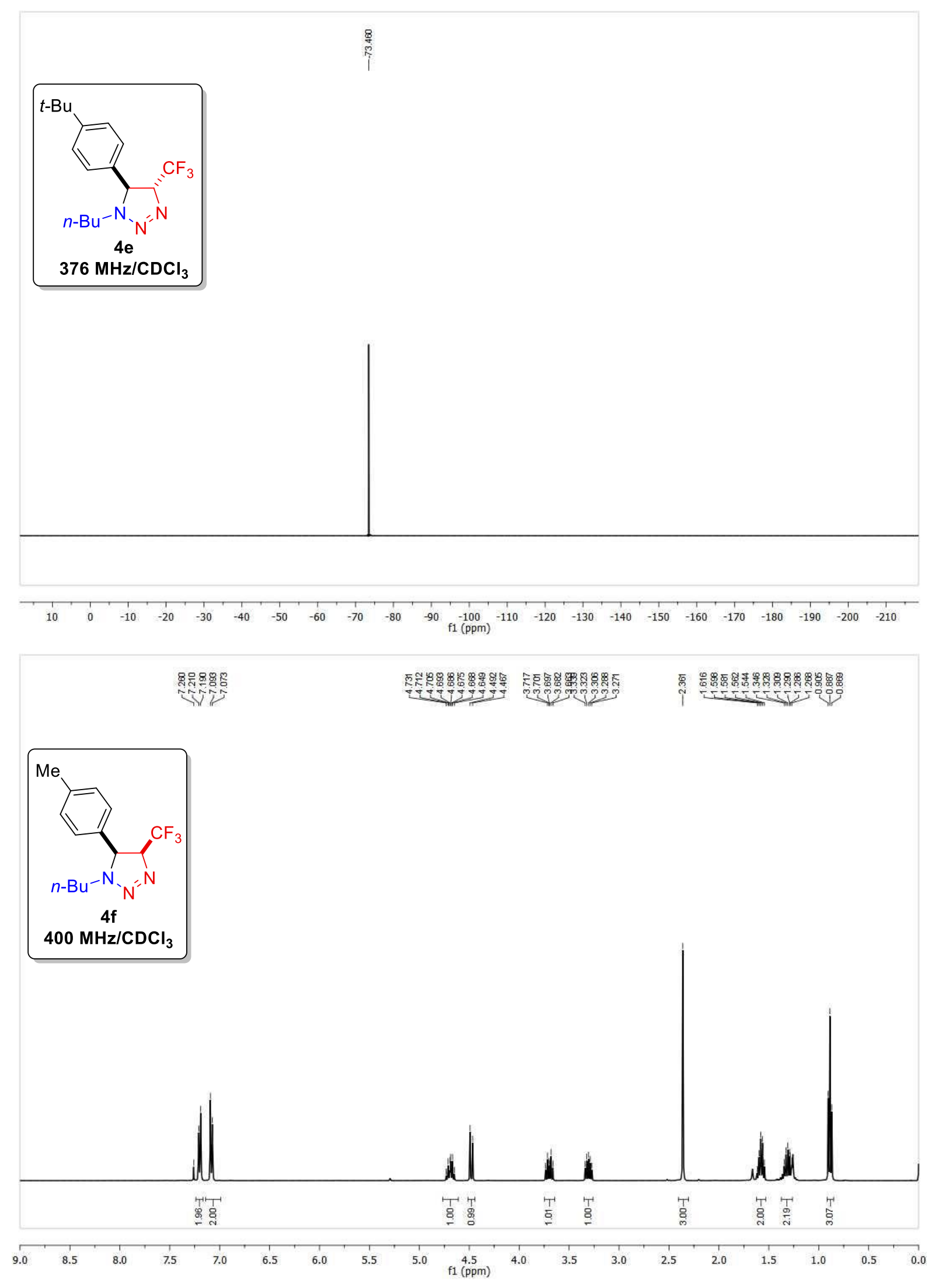



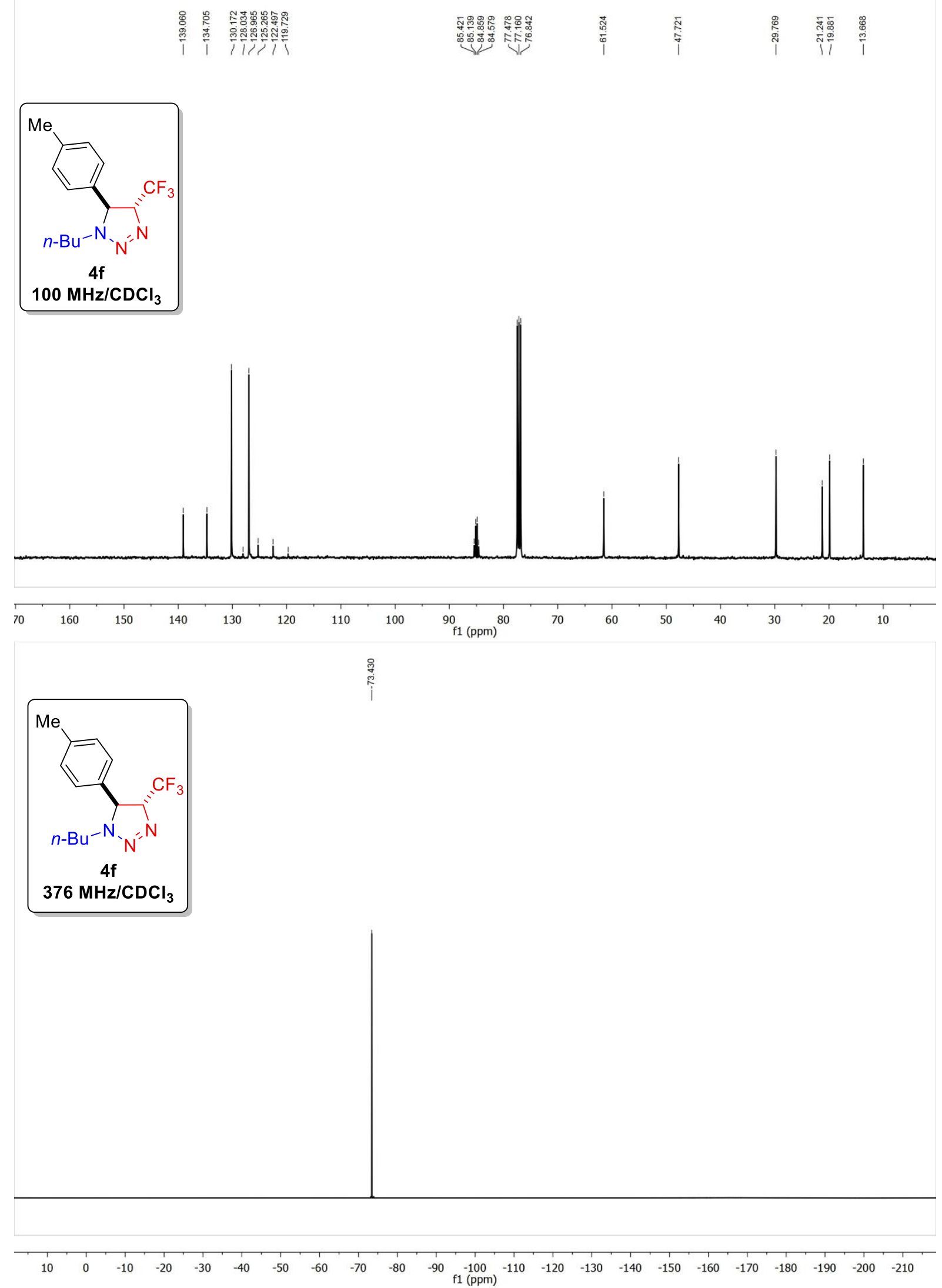

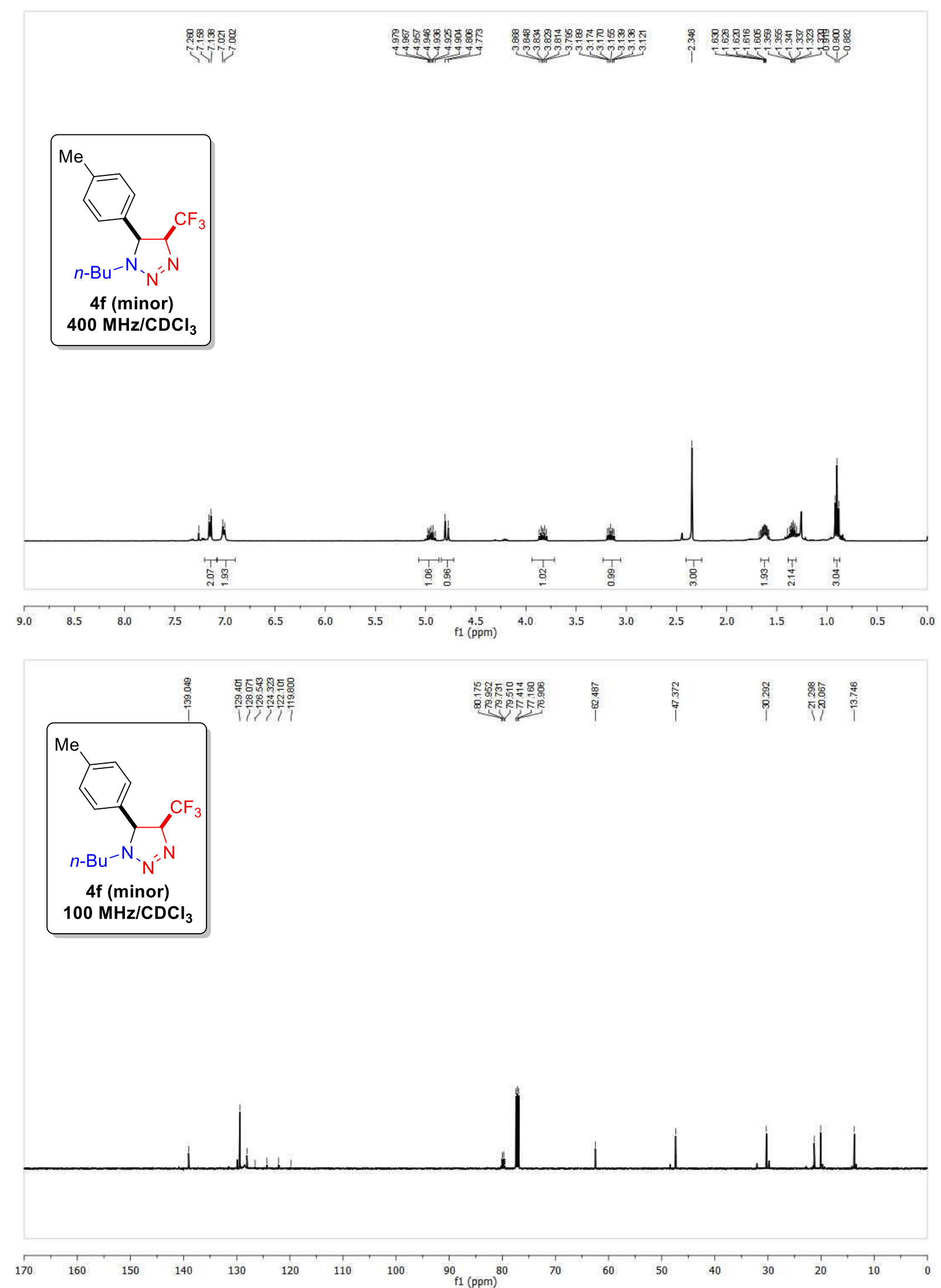

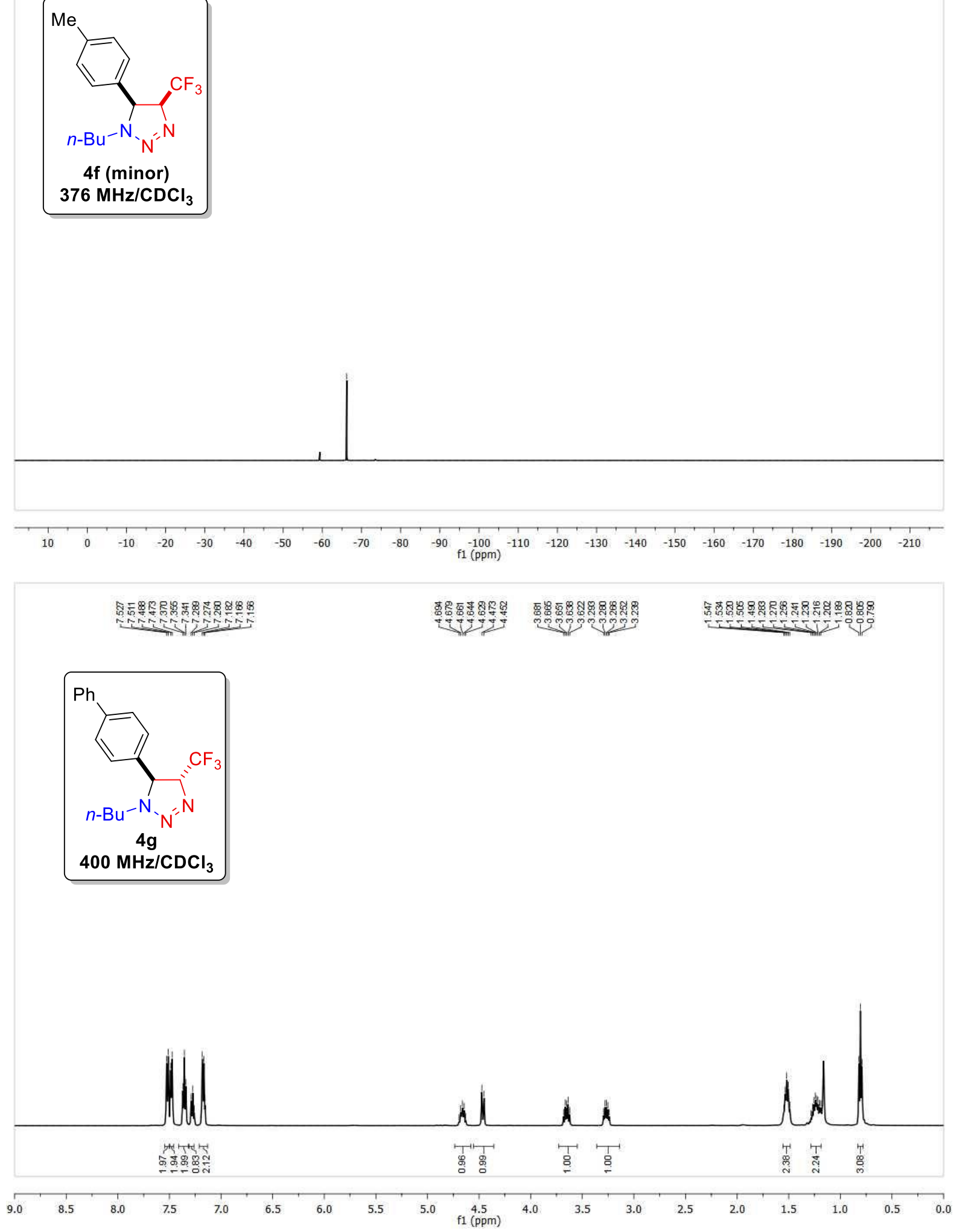

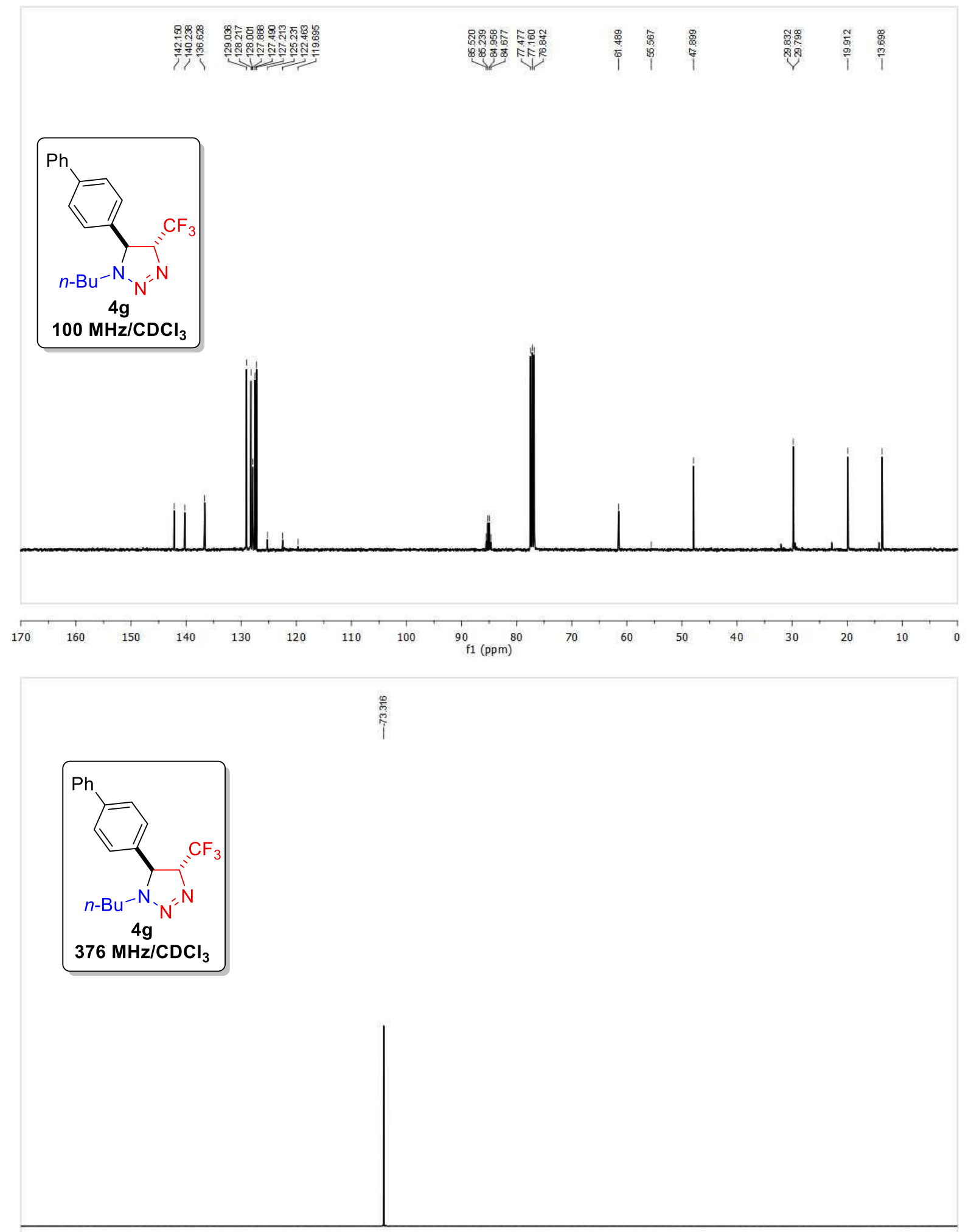

$\begin{array}{llllllllllllllllllllllll}1 & 10 & 0 & -10 & -20 & -30 & -40 & -50 & -60 & -70 & -80 & -90 & -100 & -110 & -120 & -130 & -140 & -150 & -160 & -170 & -180 & -190 & -200 & -210\end{array}$ 

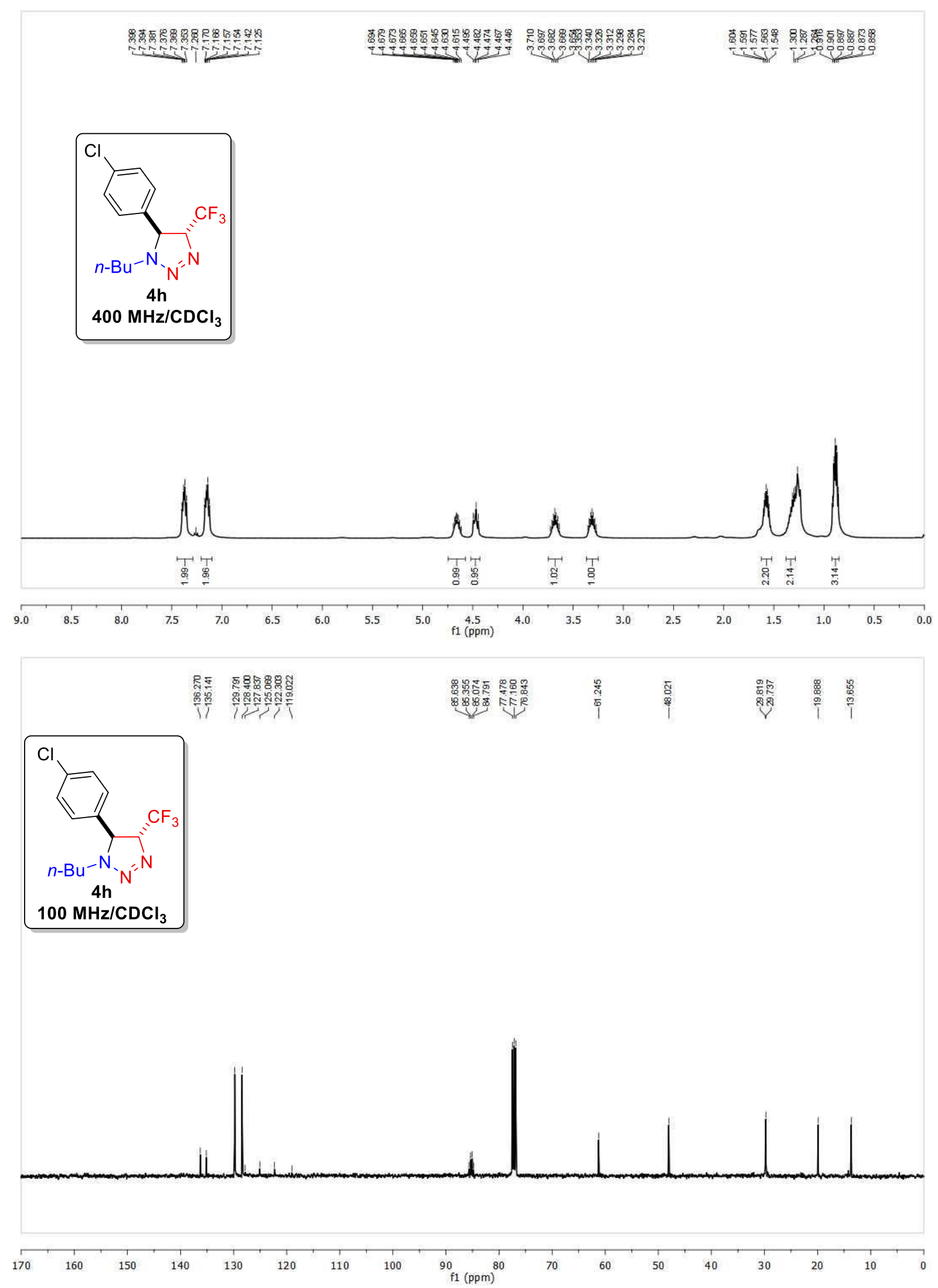


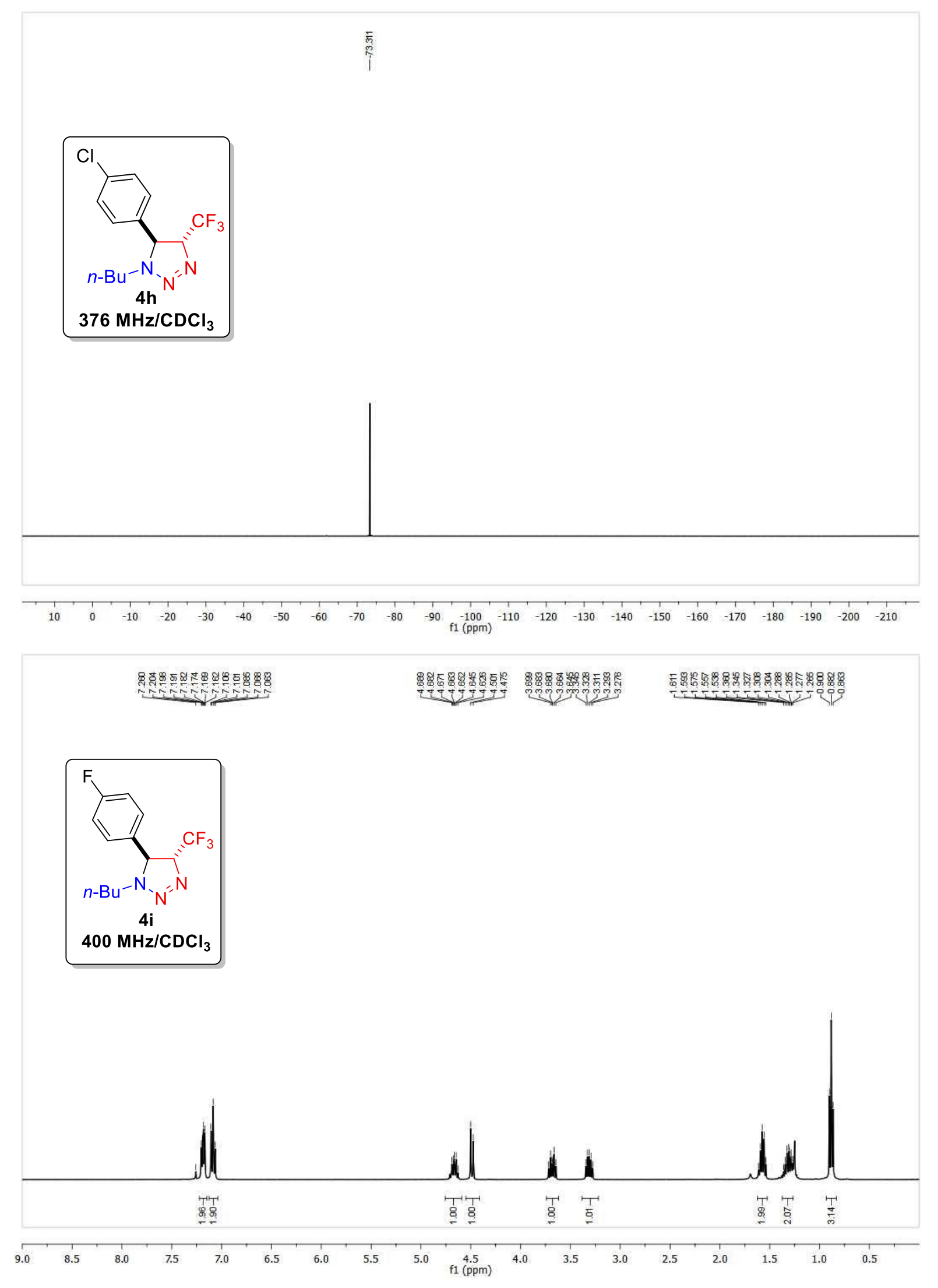



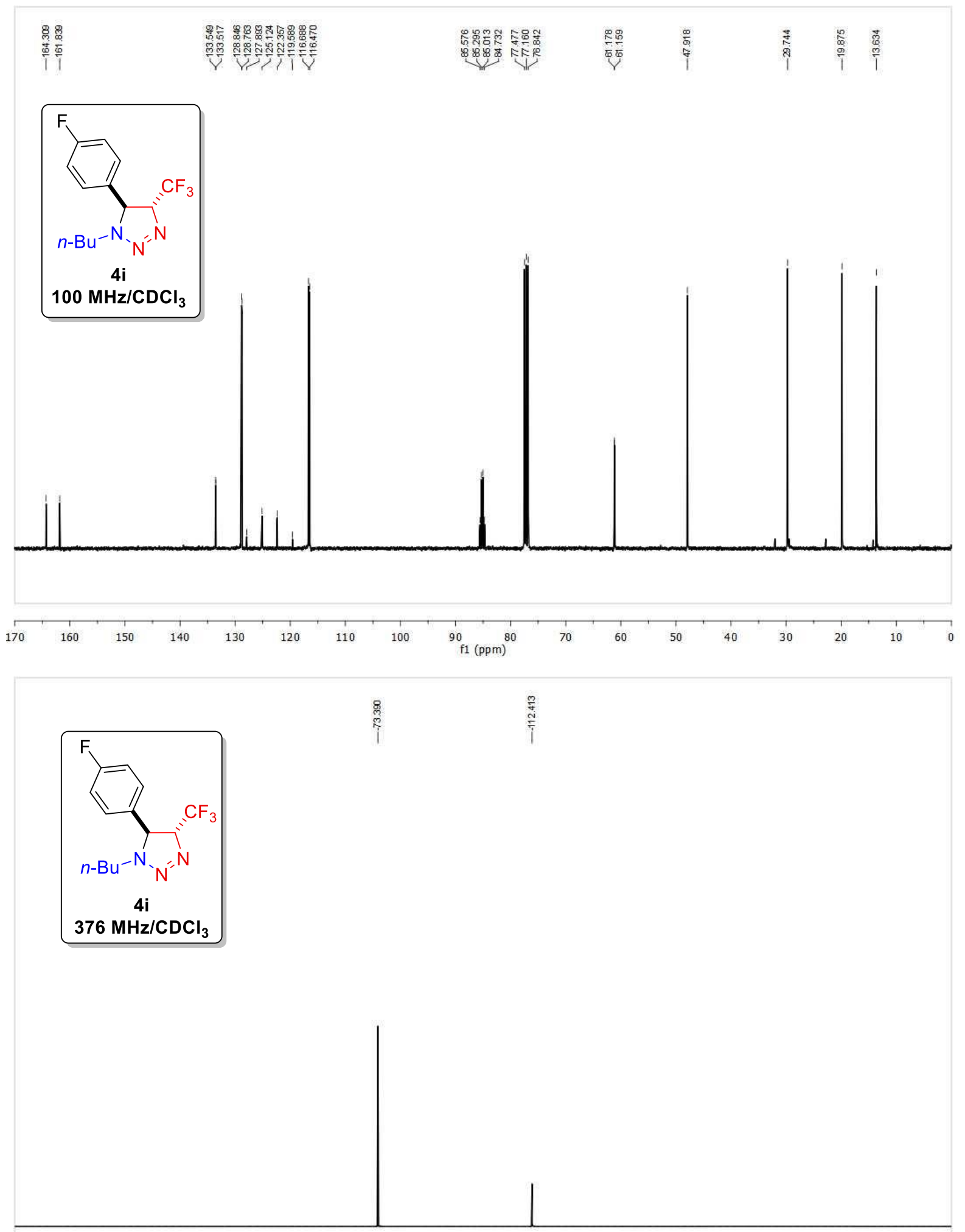

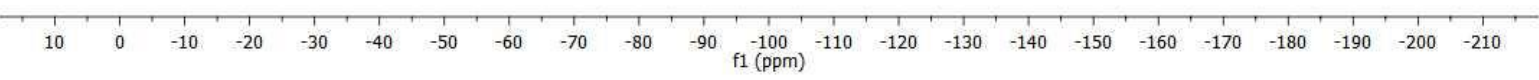



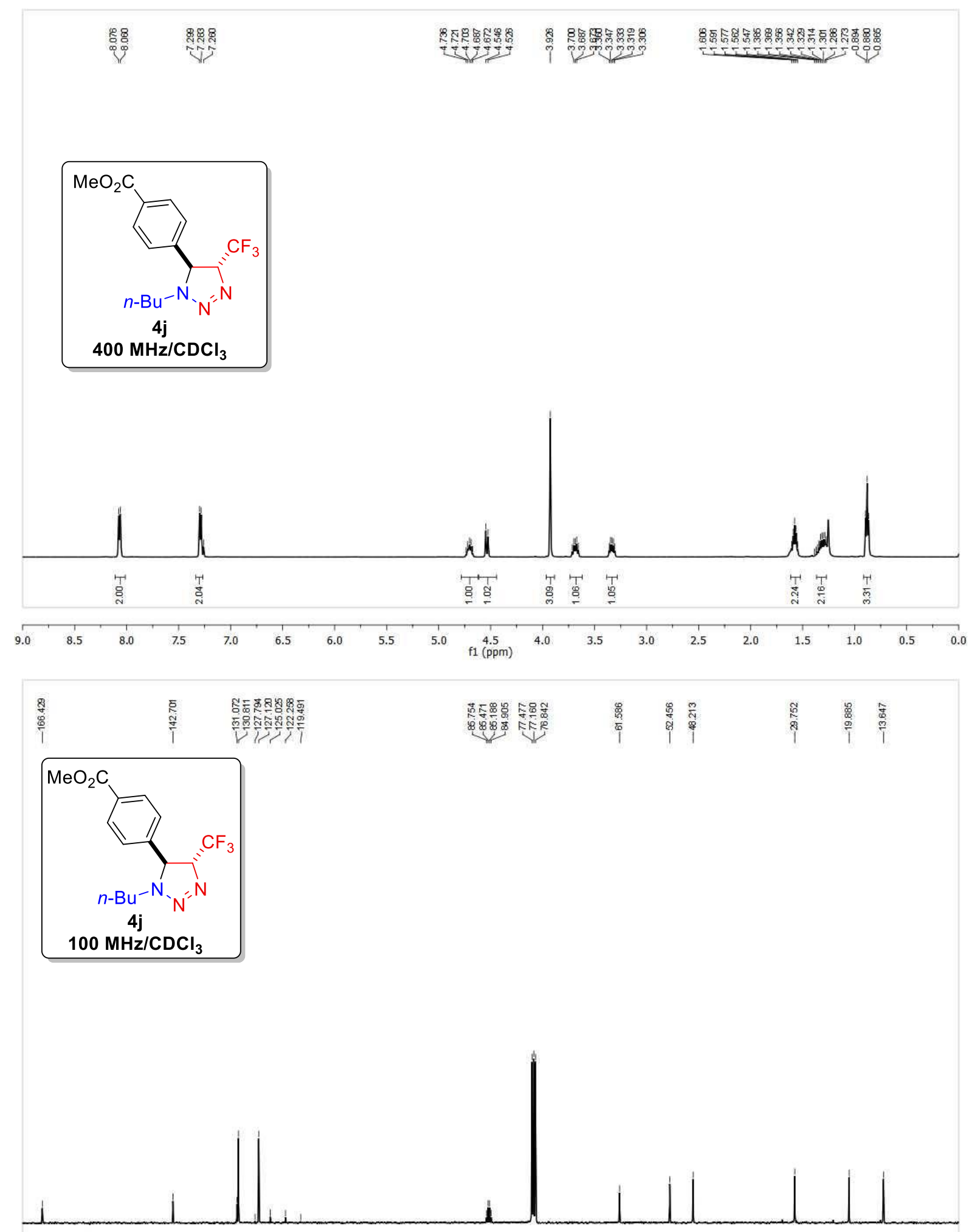

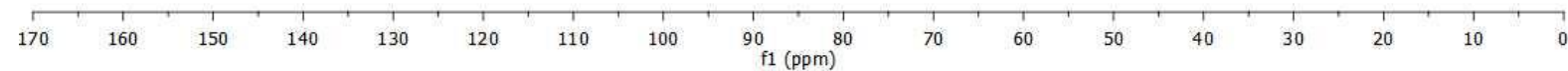


$\mathrm{MeO}_{2} \mathrm{C}$

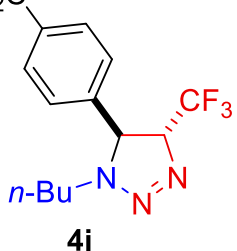

$4 j$

$376 \mathrm{MHz} / \mathrm{CDCl}_{3}$

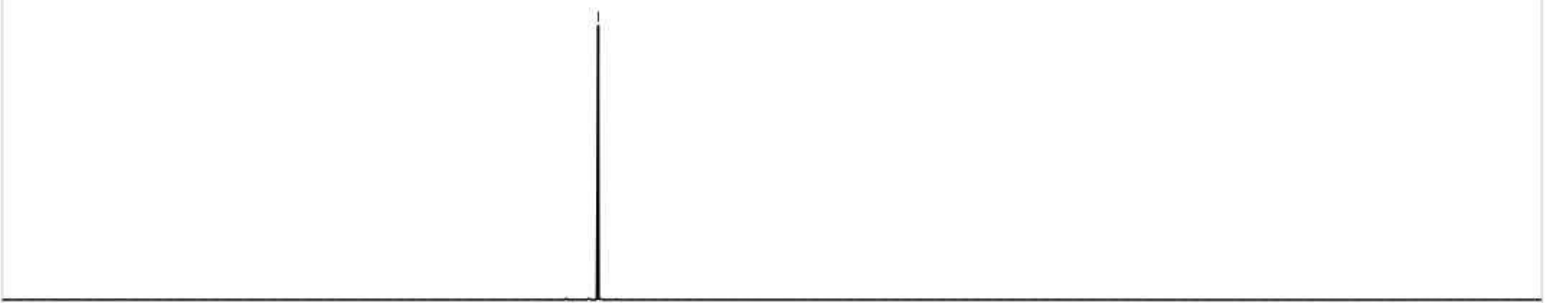

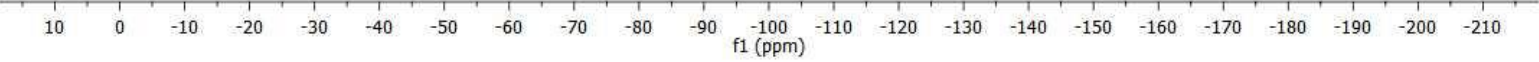

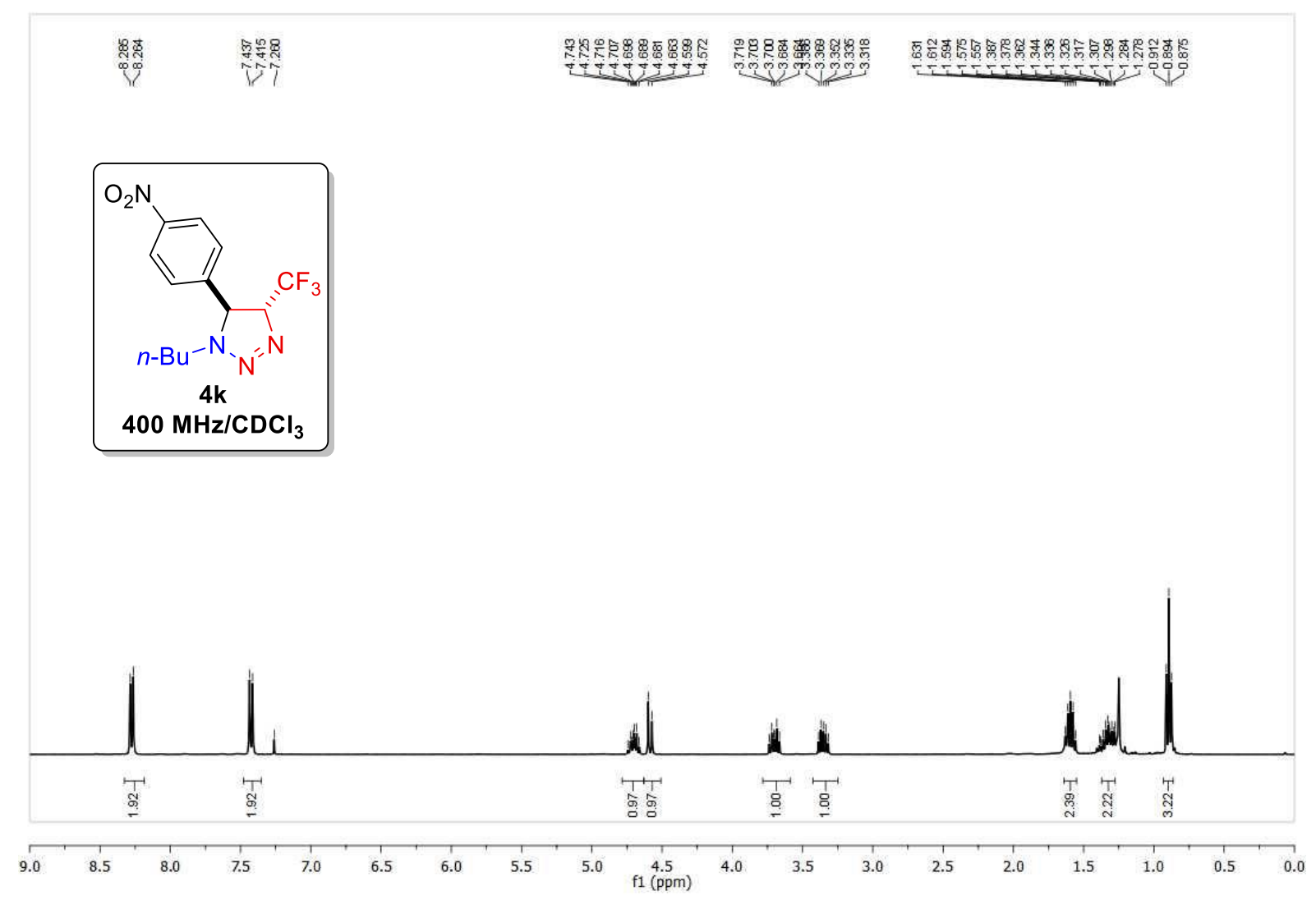



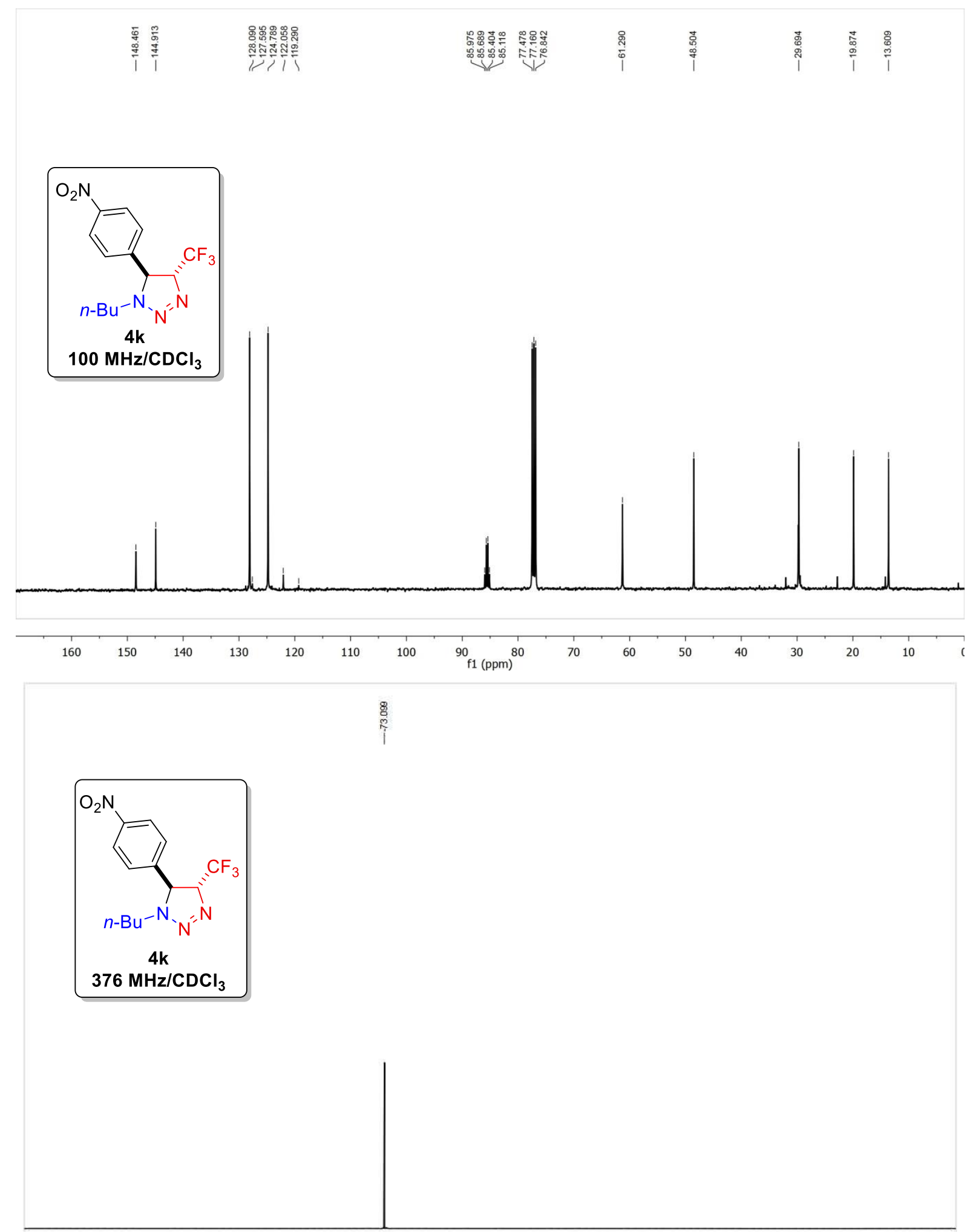

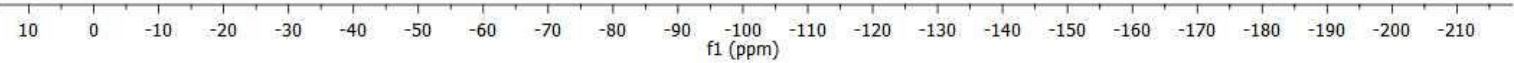



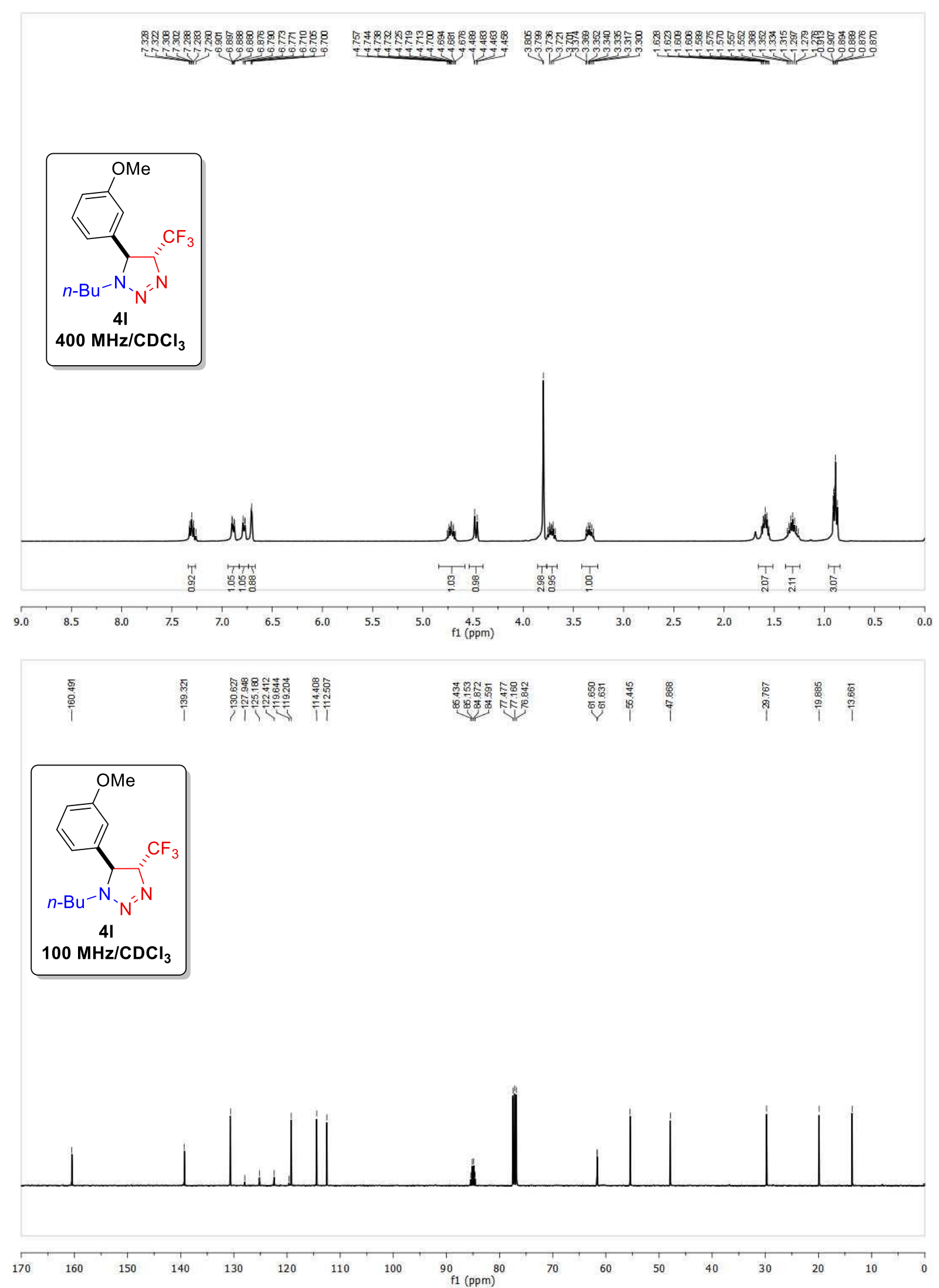


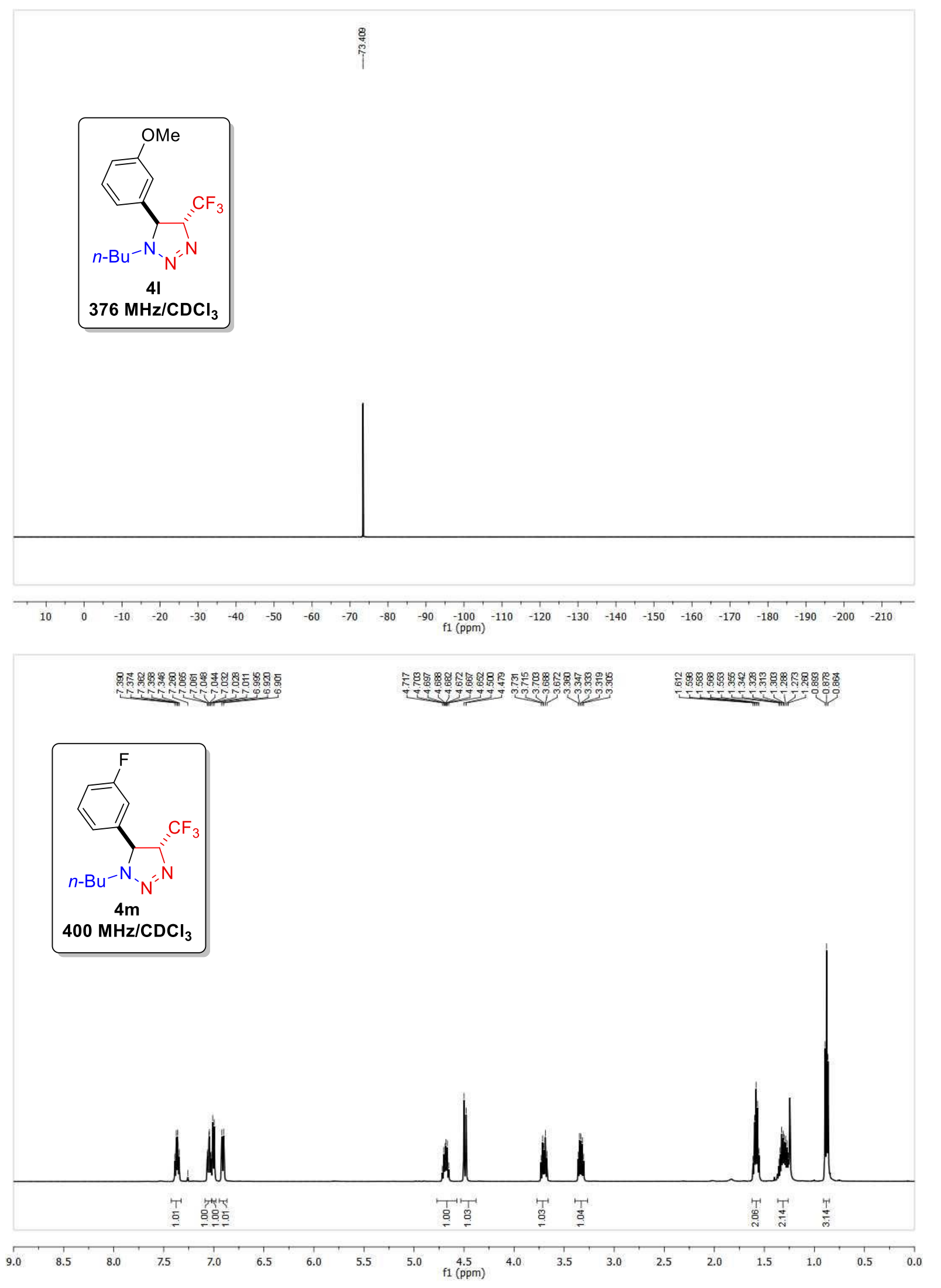



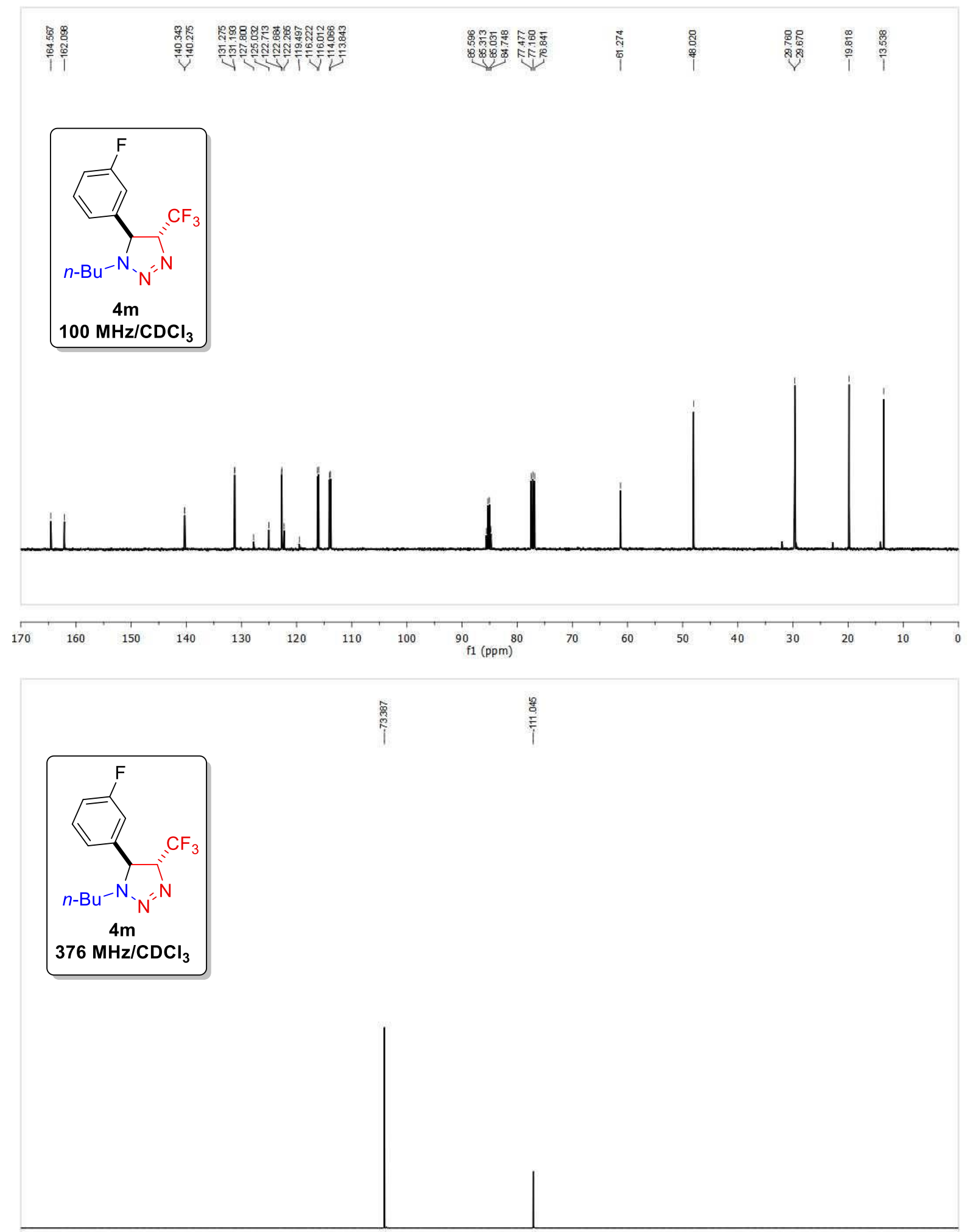

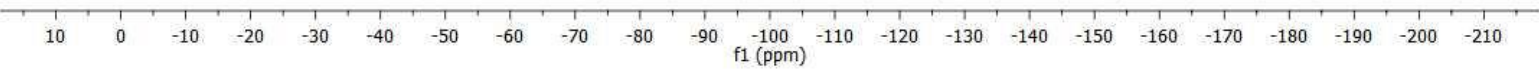




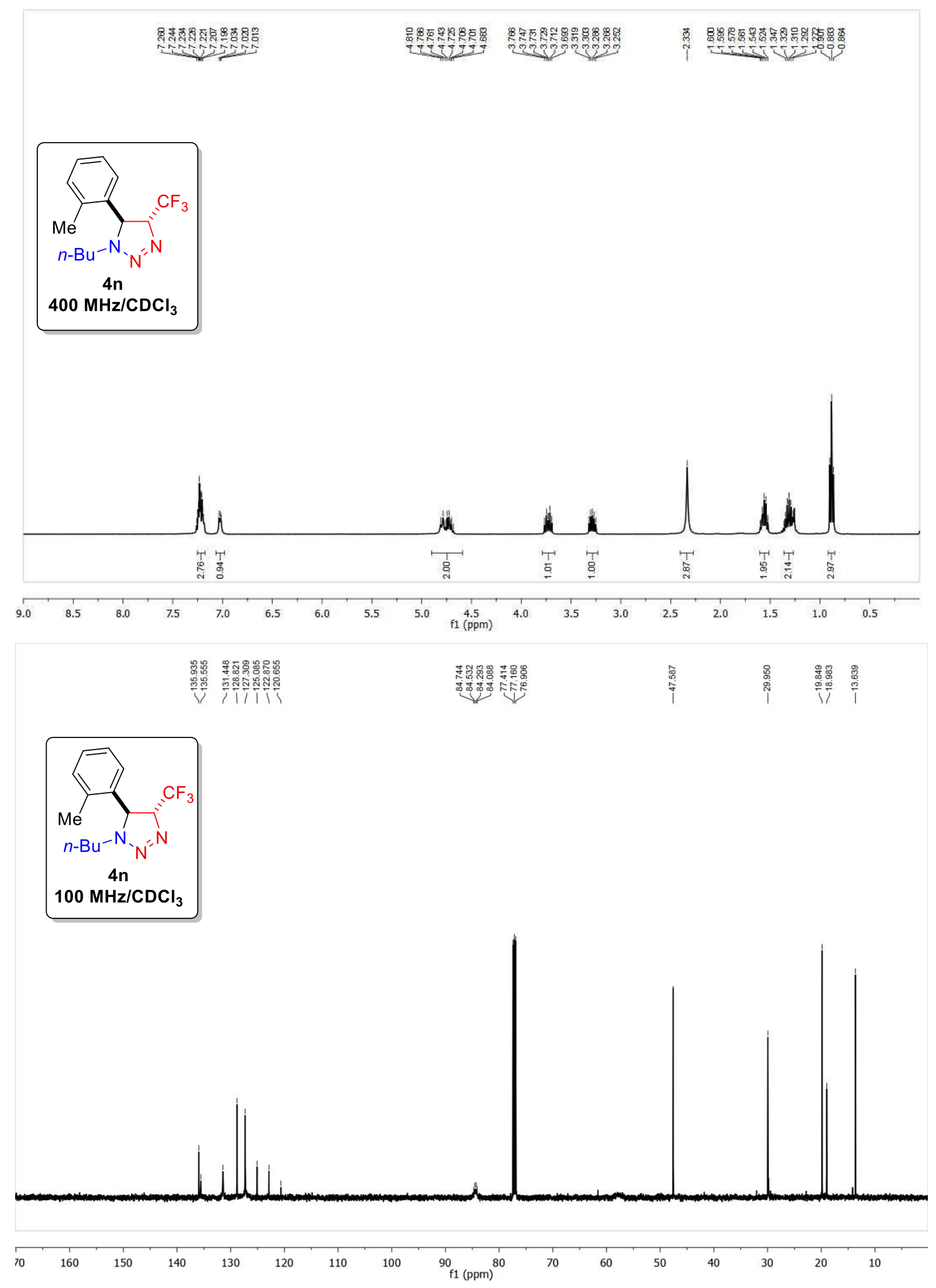




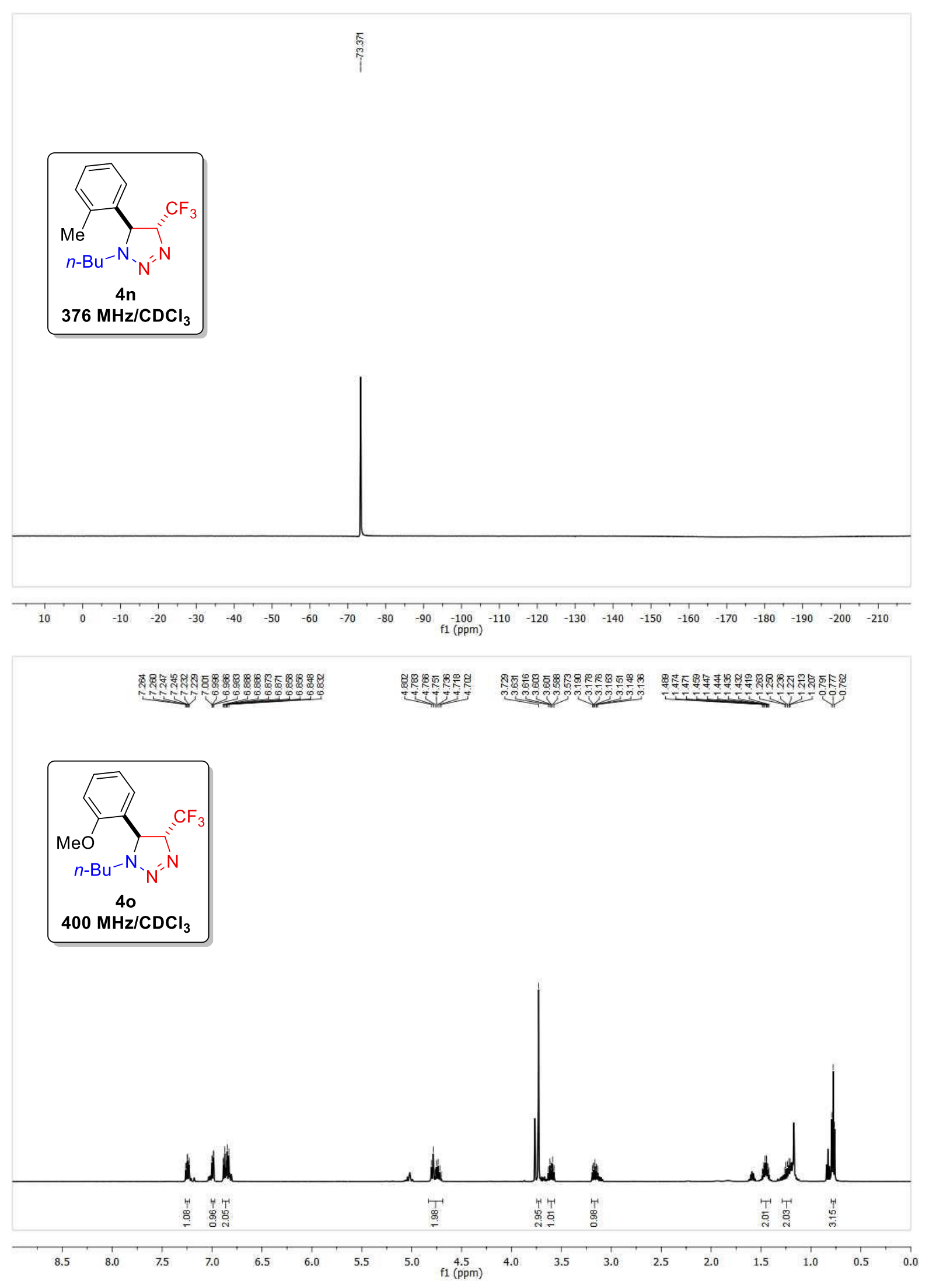




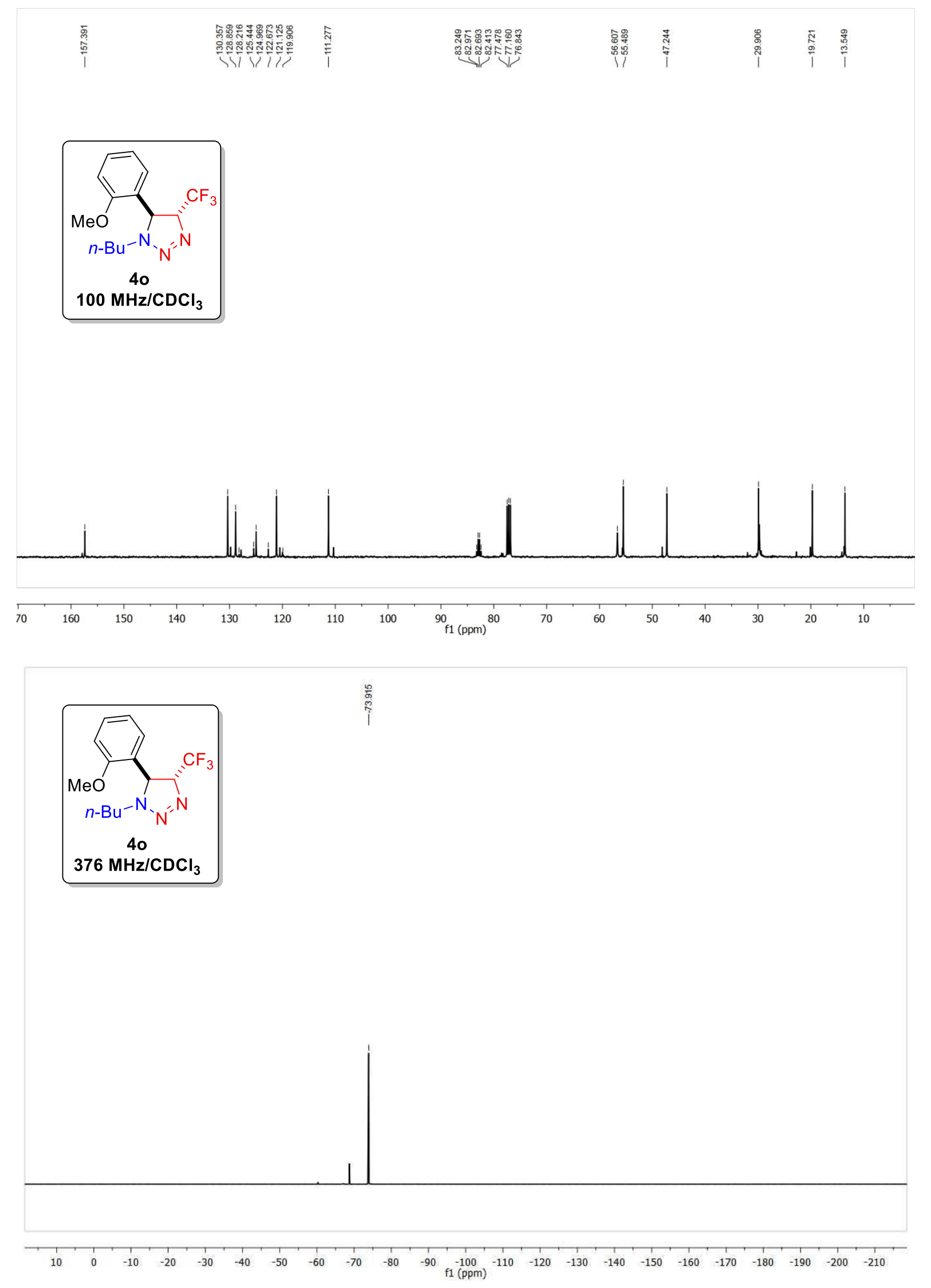



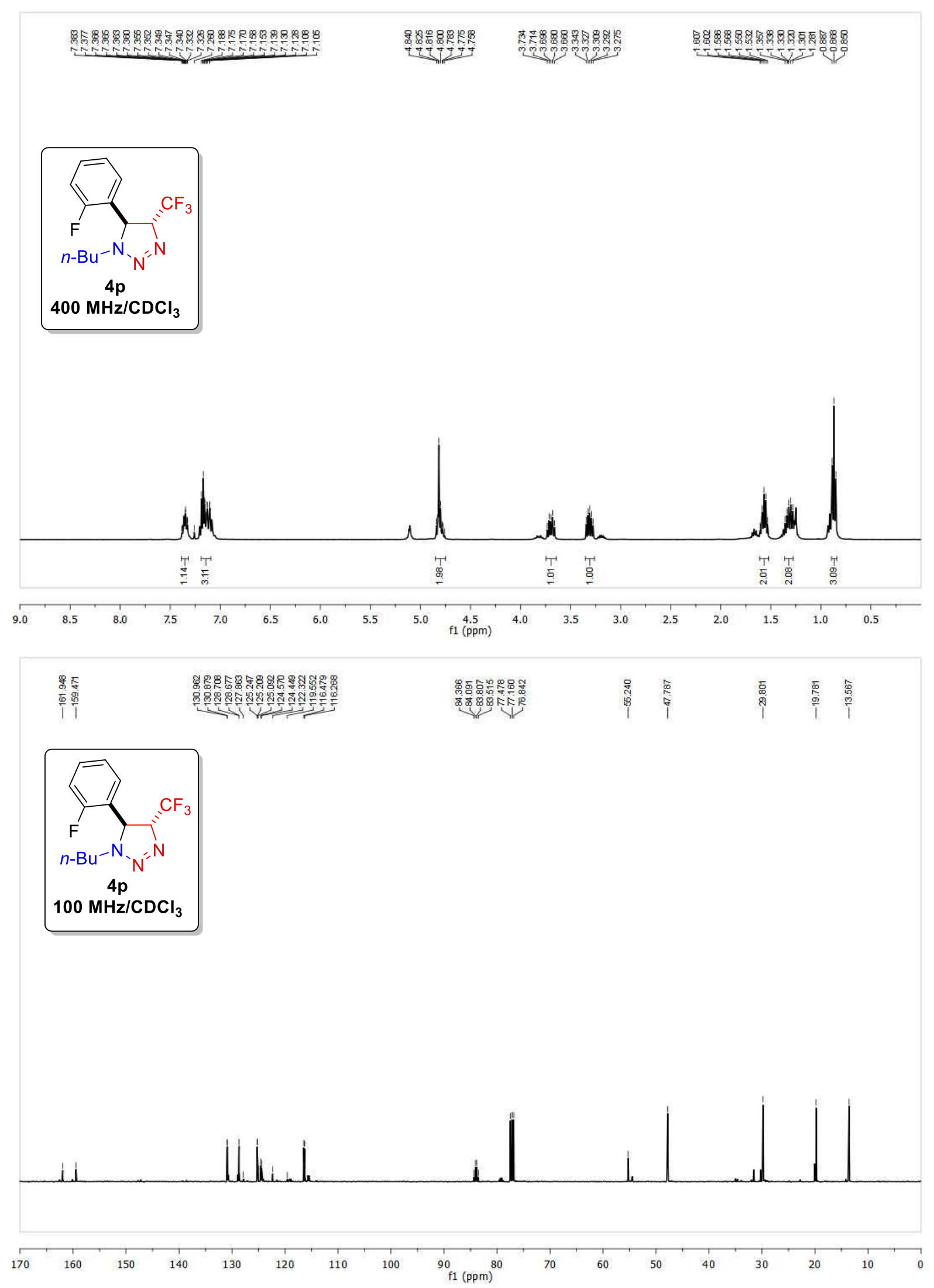


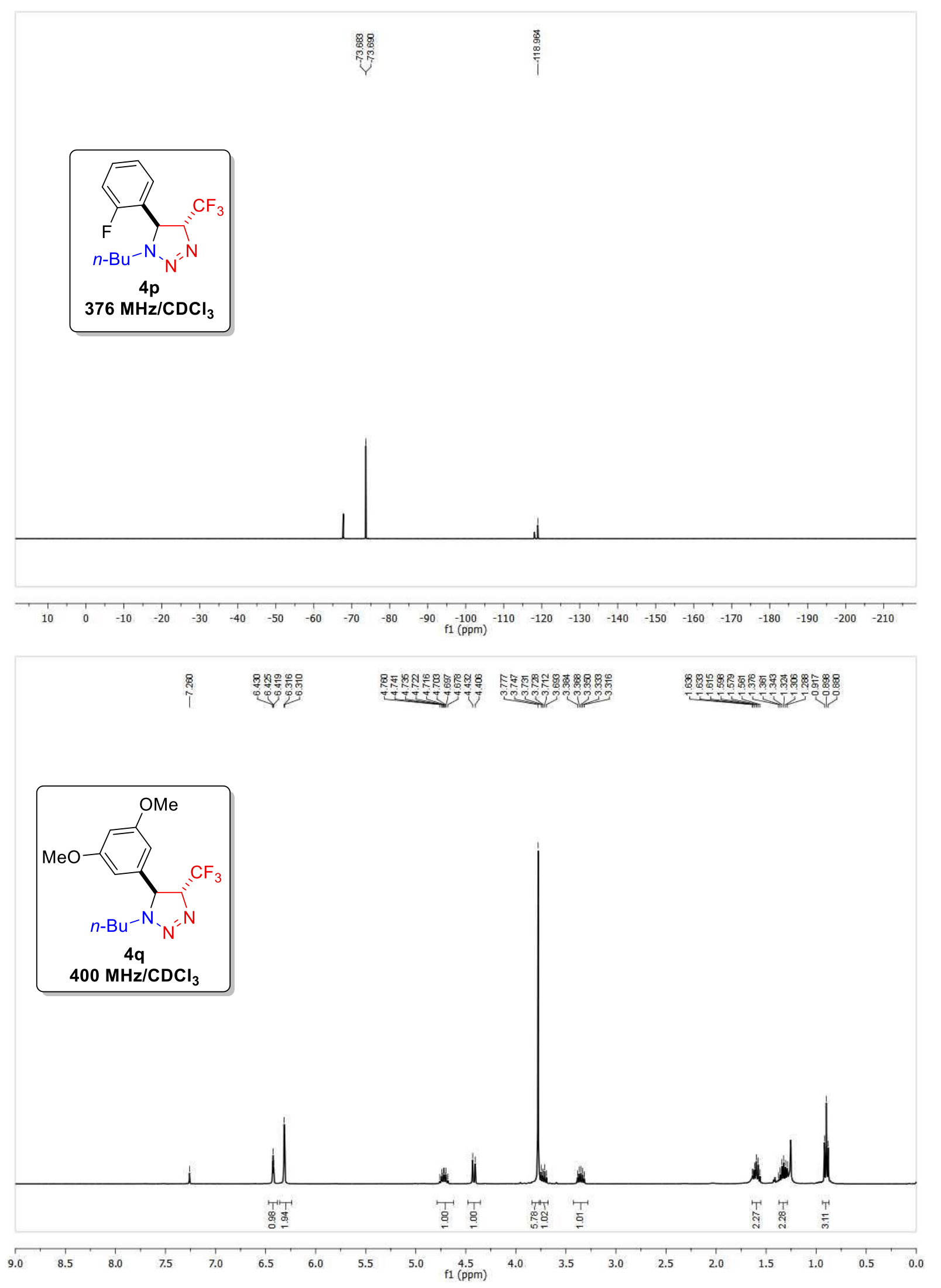



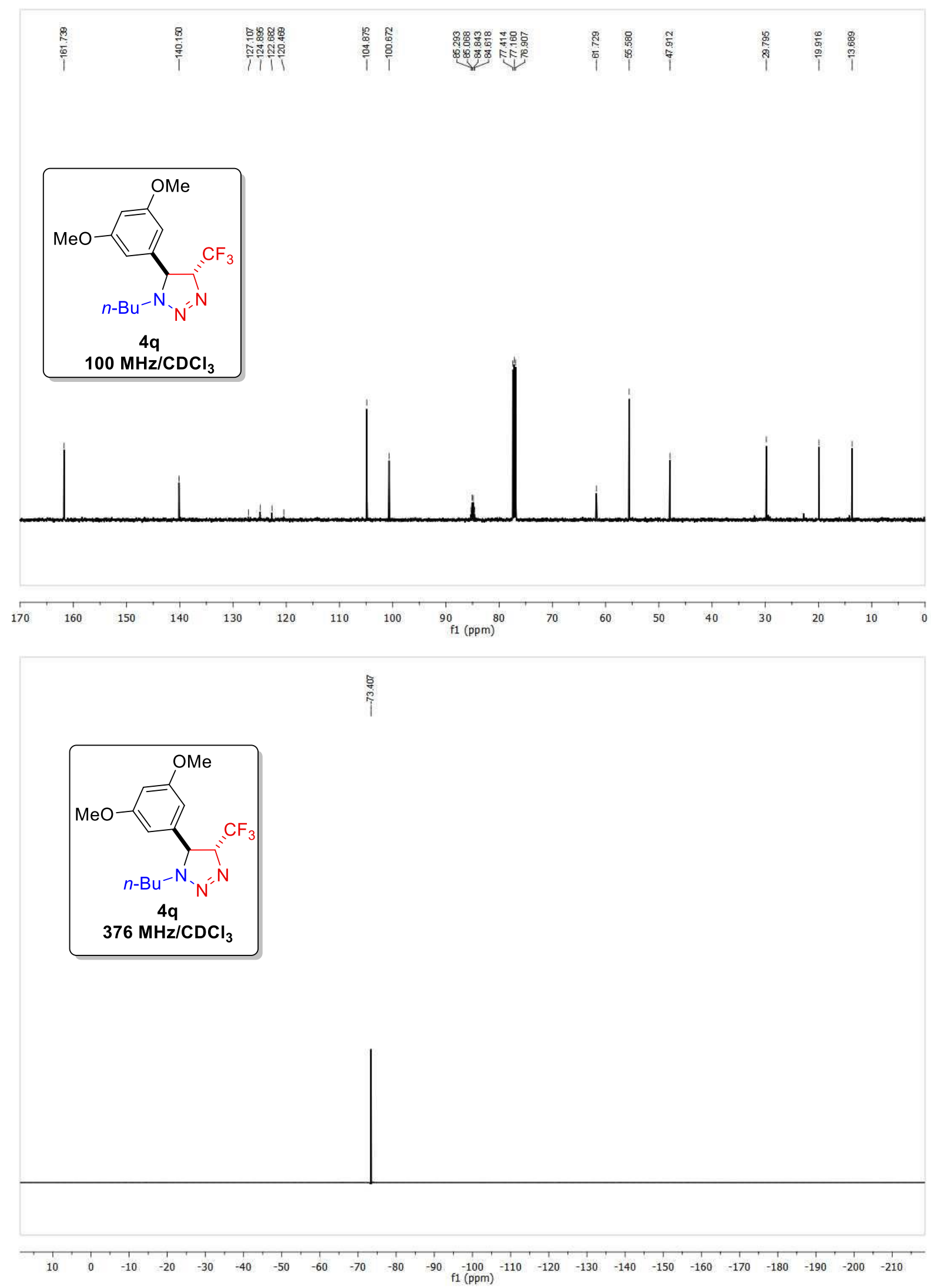


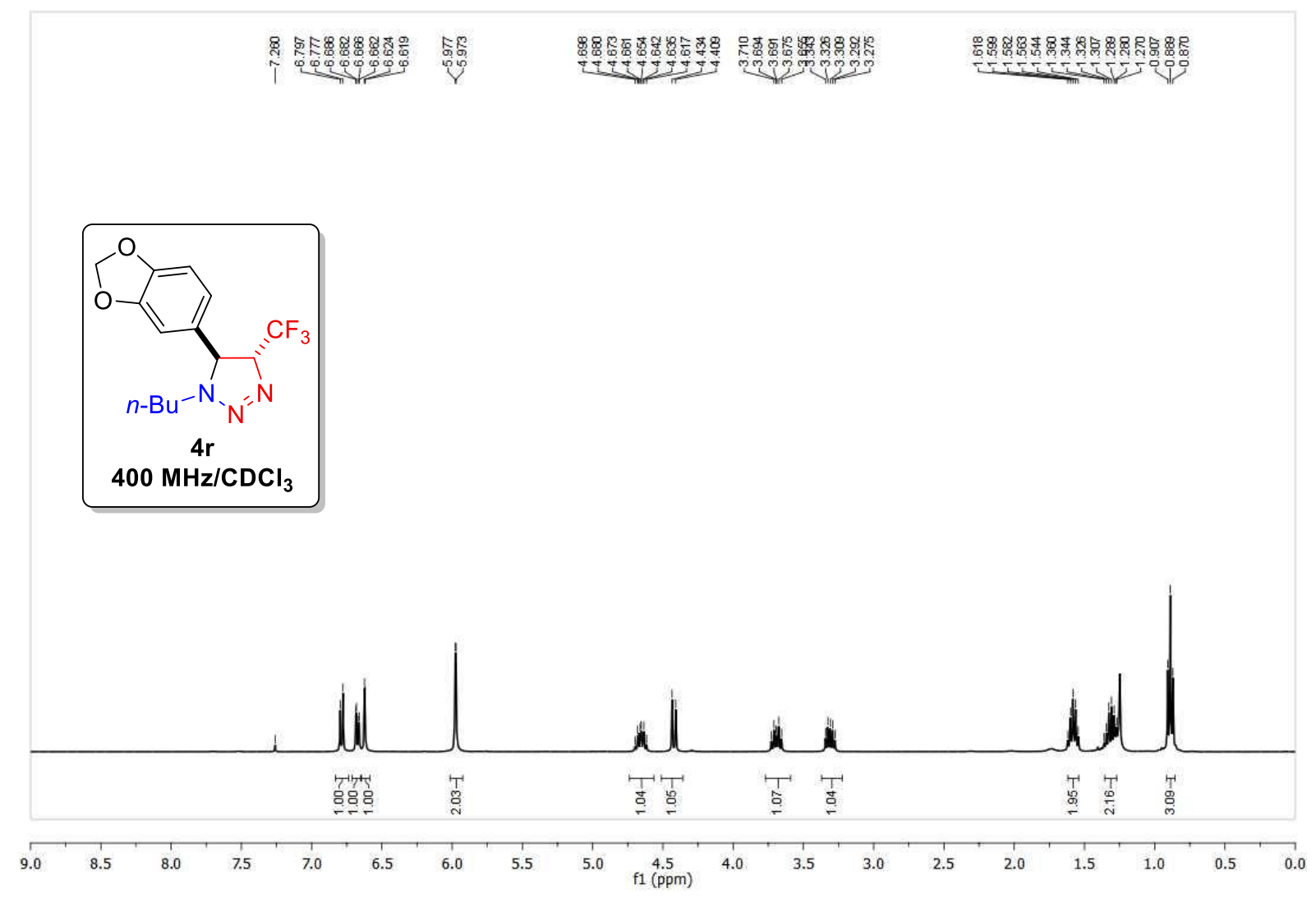

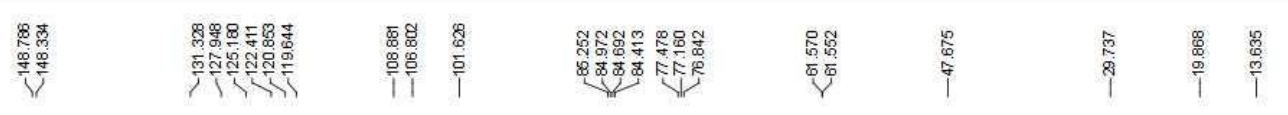
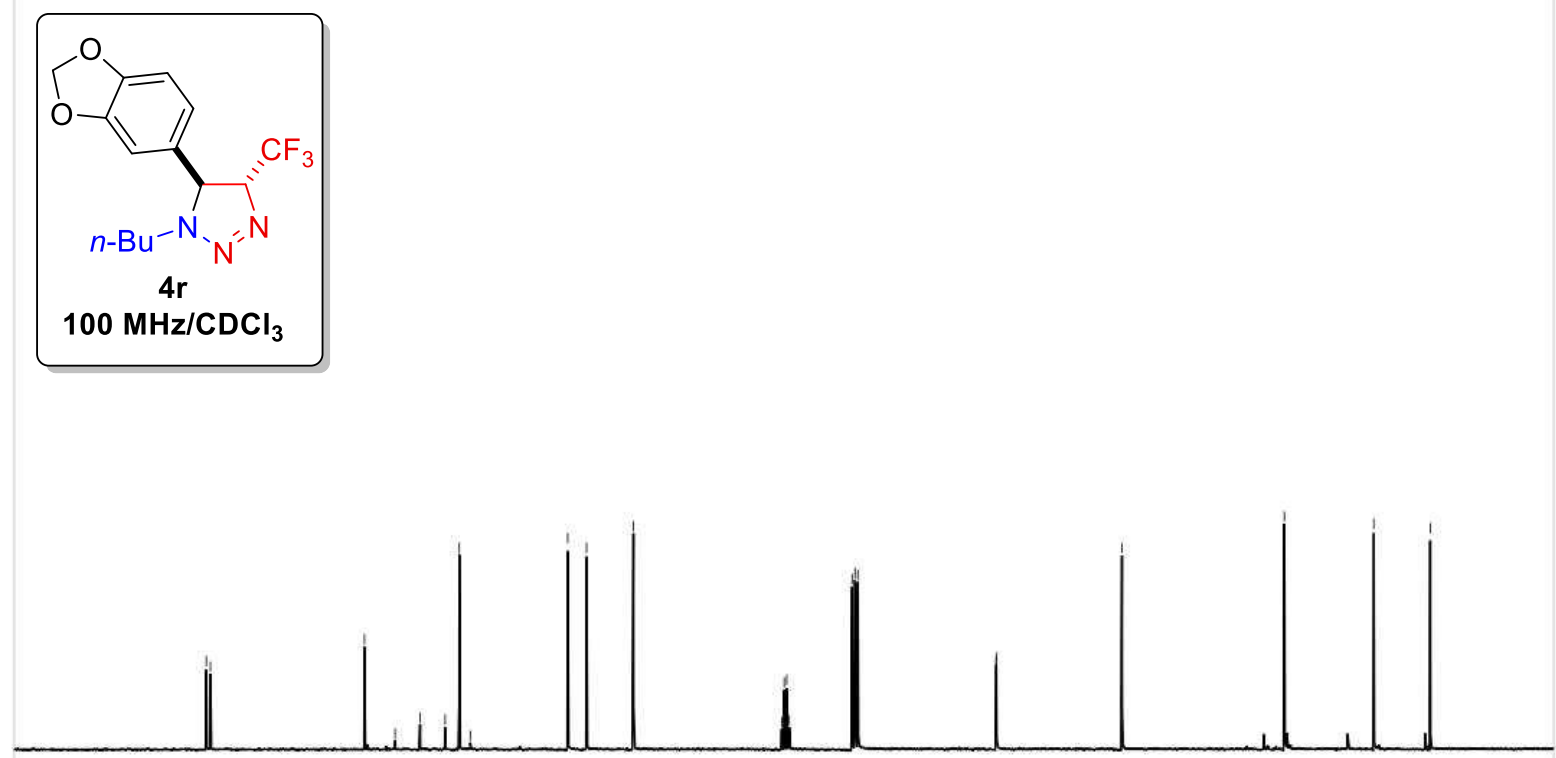

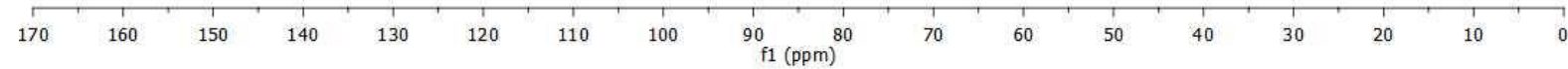




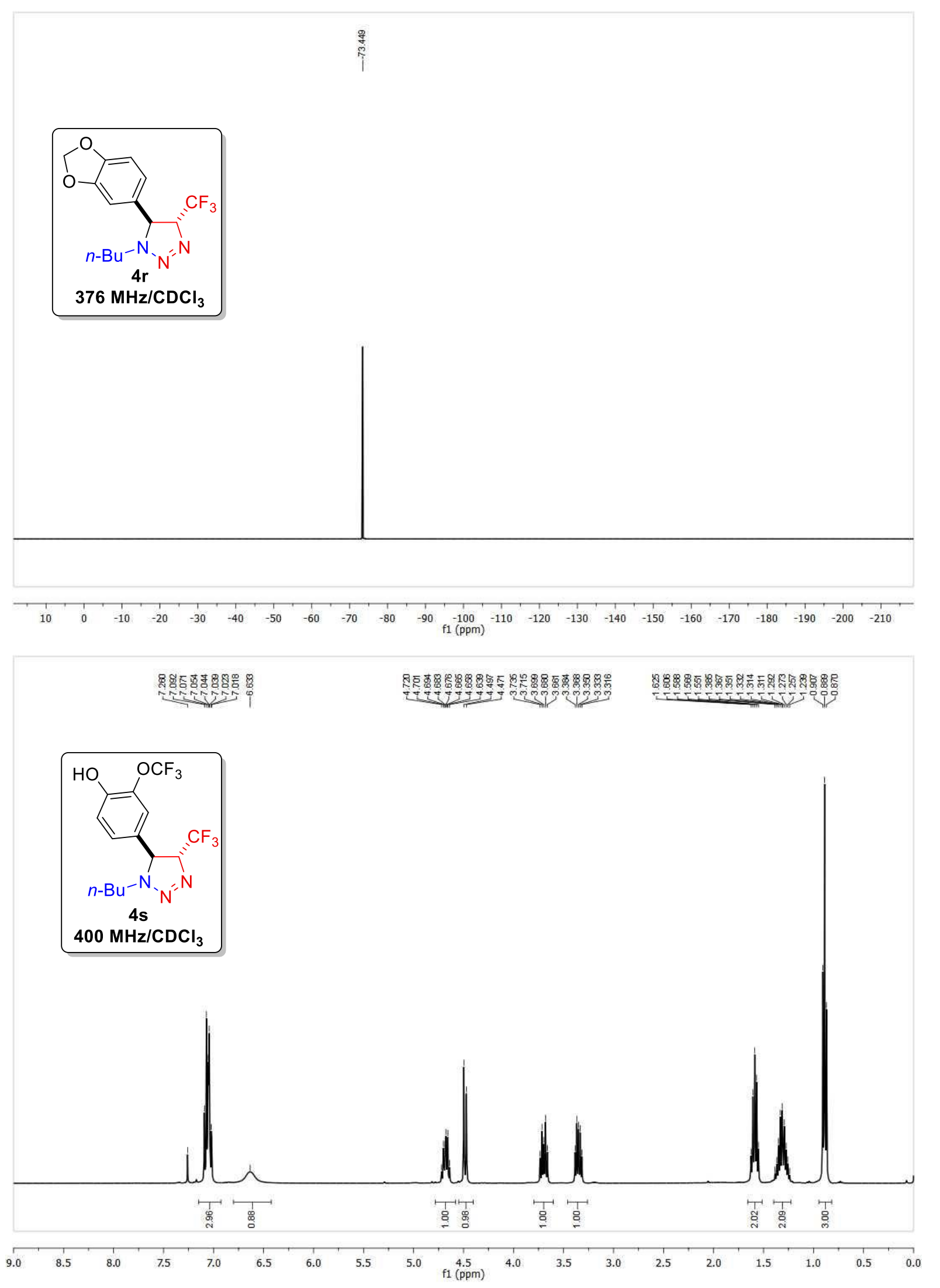



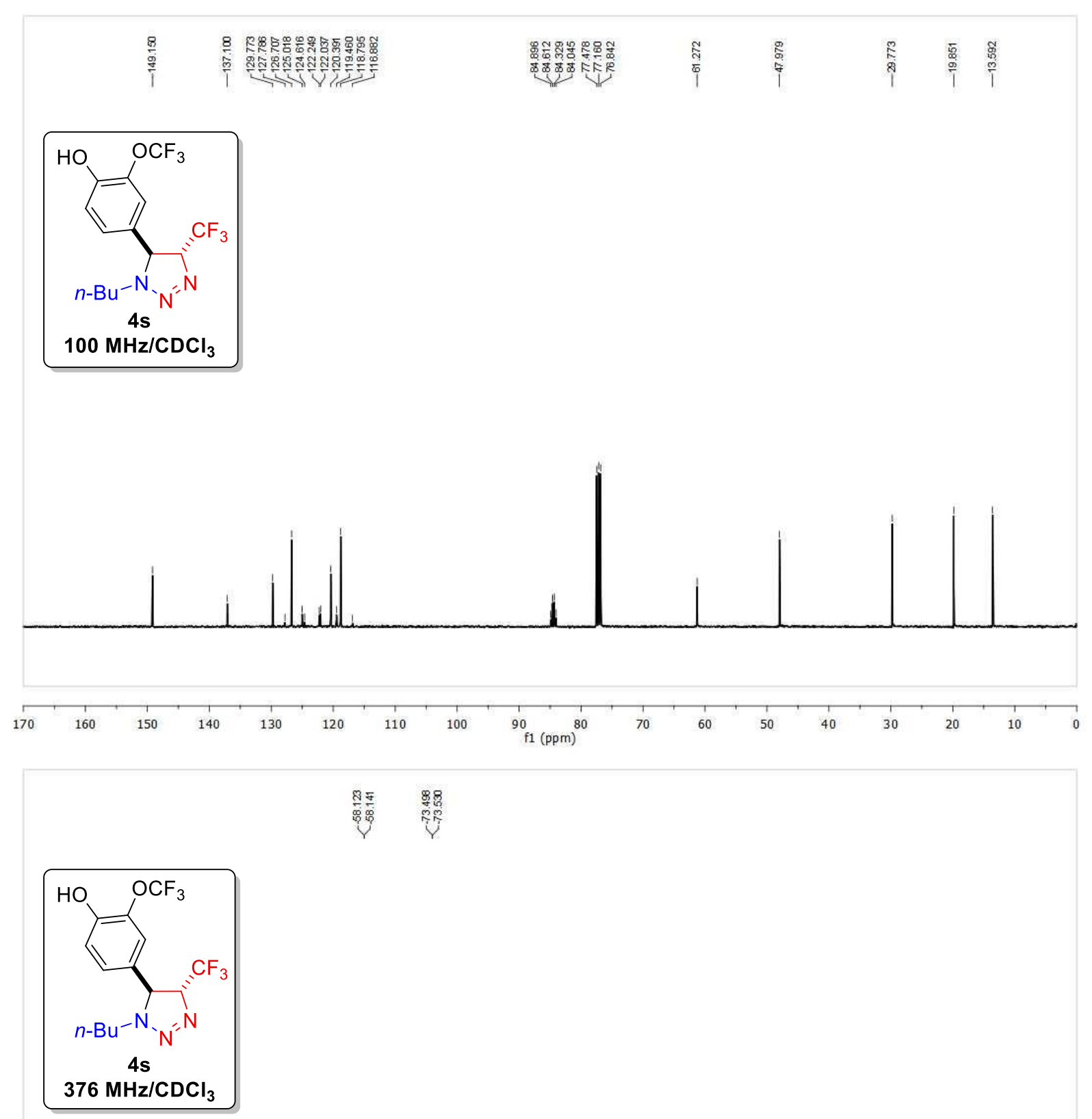

Yे

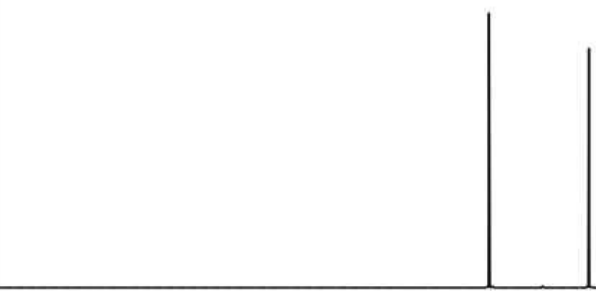

$\begin{array}{rlllllllllllllllllllllll}1 & 10 & 0 & -10 & -20 & -30 & -40 & -50 & -60 & -70 & -80 & -90 & -100 & -110 & -120 & -130 & -140 & -150 & -160 & -170 & -180 & -190 & -200 & -210\end{array}$ 

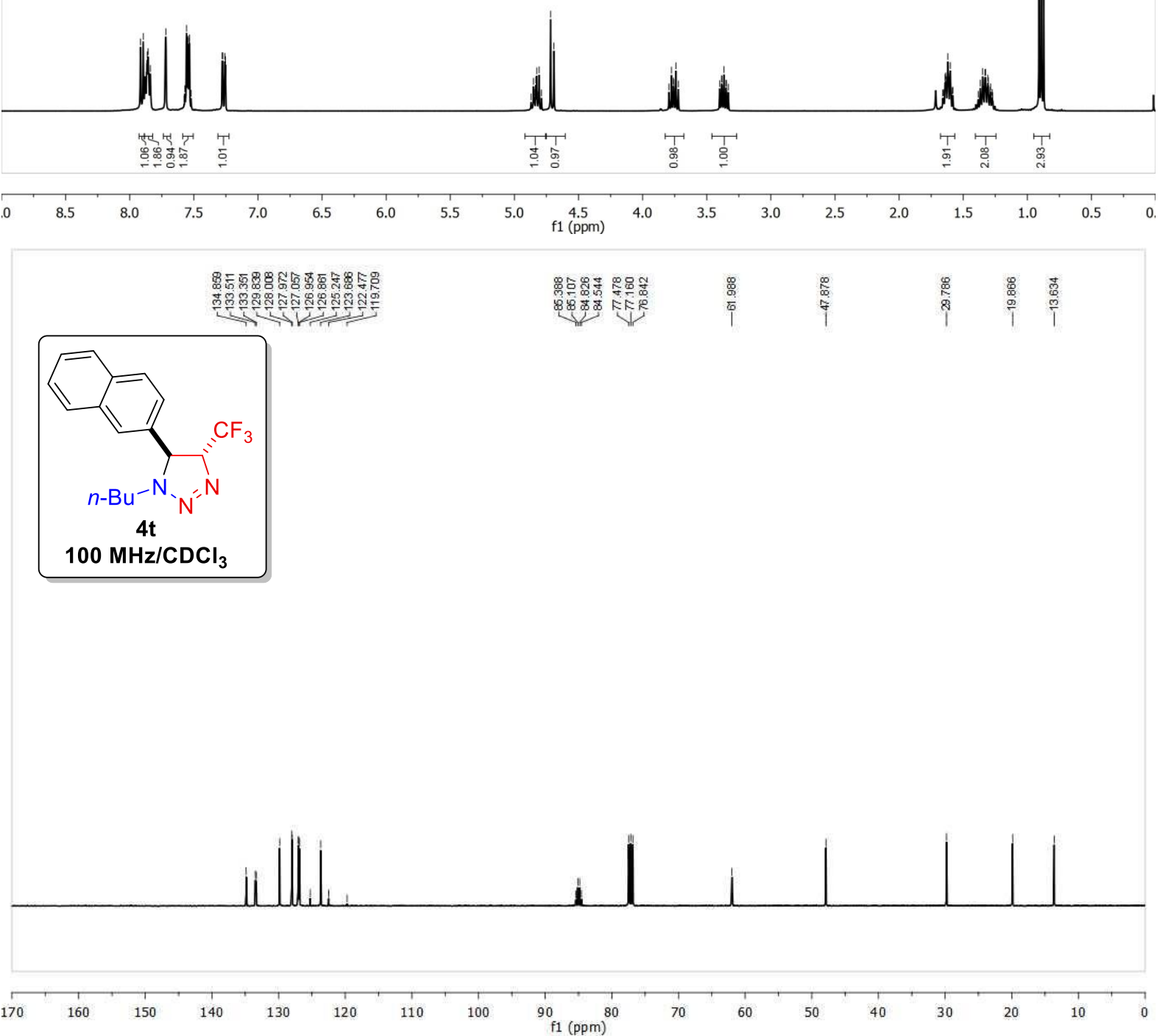

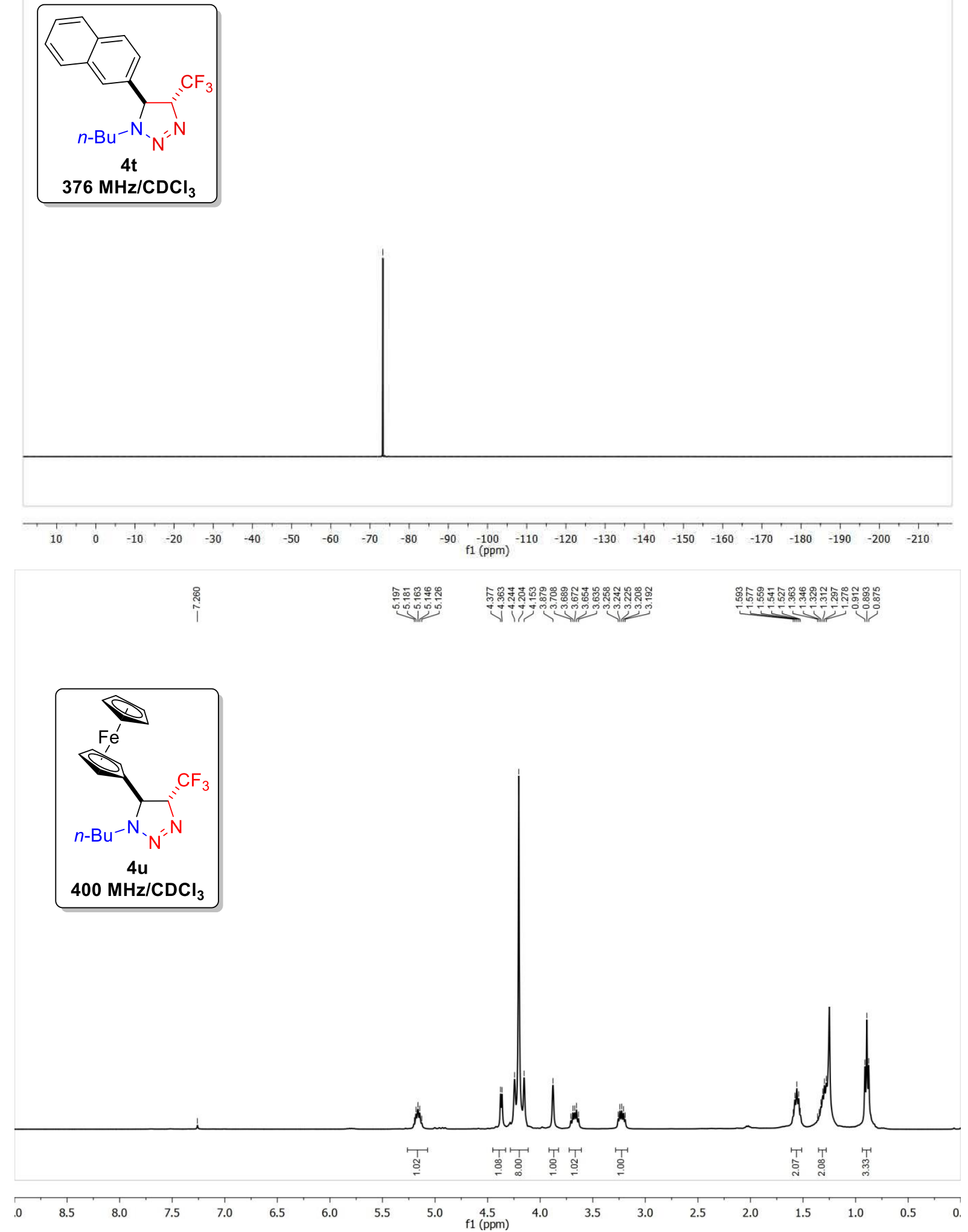

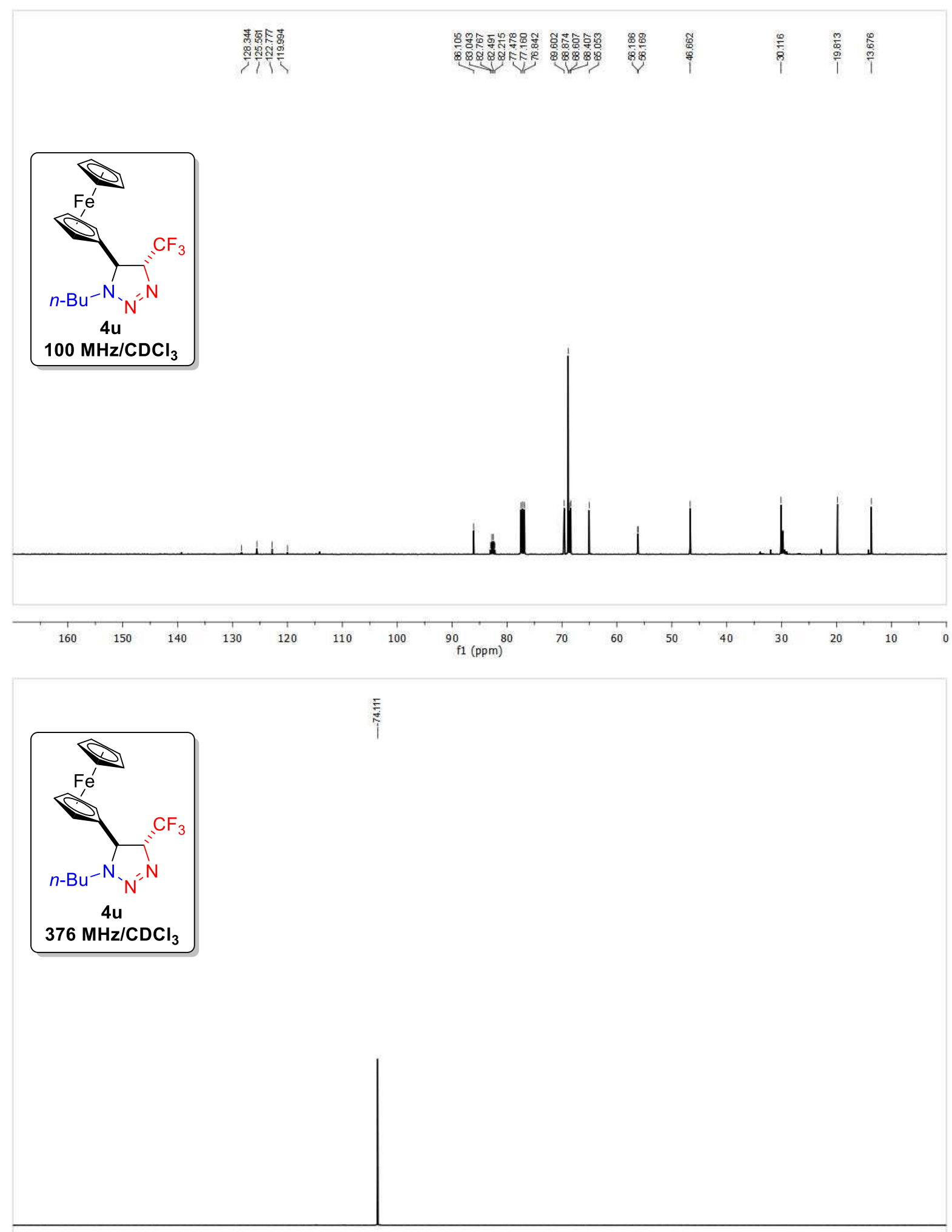

$\begin{array}{llllllllllllllllllllllll}1 & 10 & 0 & -10 & -20 & -30 & -40 & -50 & -60 & -70 & -80 & -90 & -100 & -110 & -120 & -130 & -140 & -150 & -160 & -170 & -180 & -190 & -200 & -210\end{array}$ 


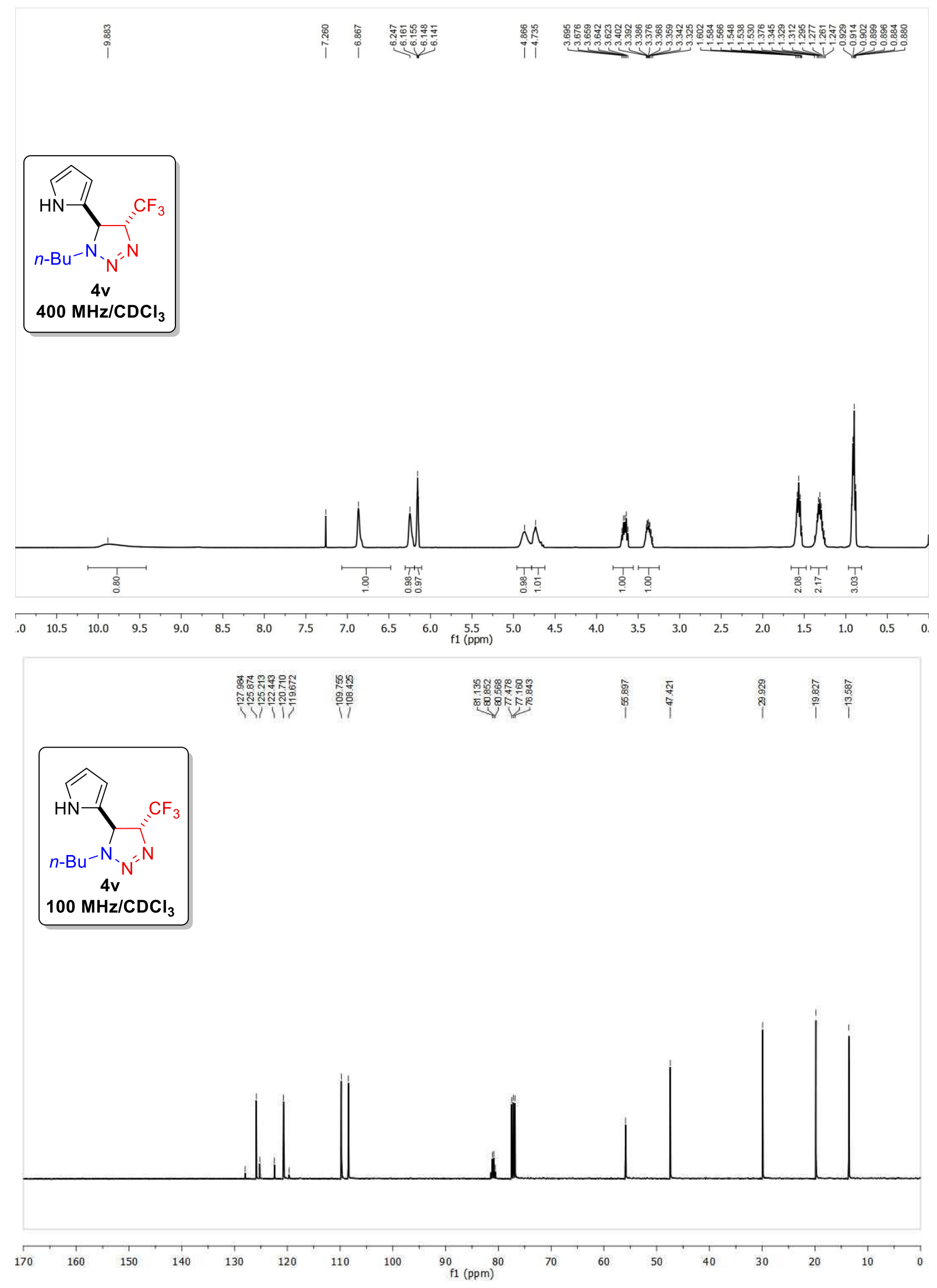




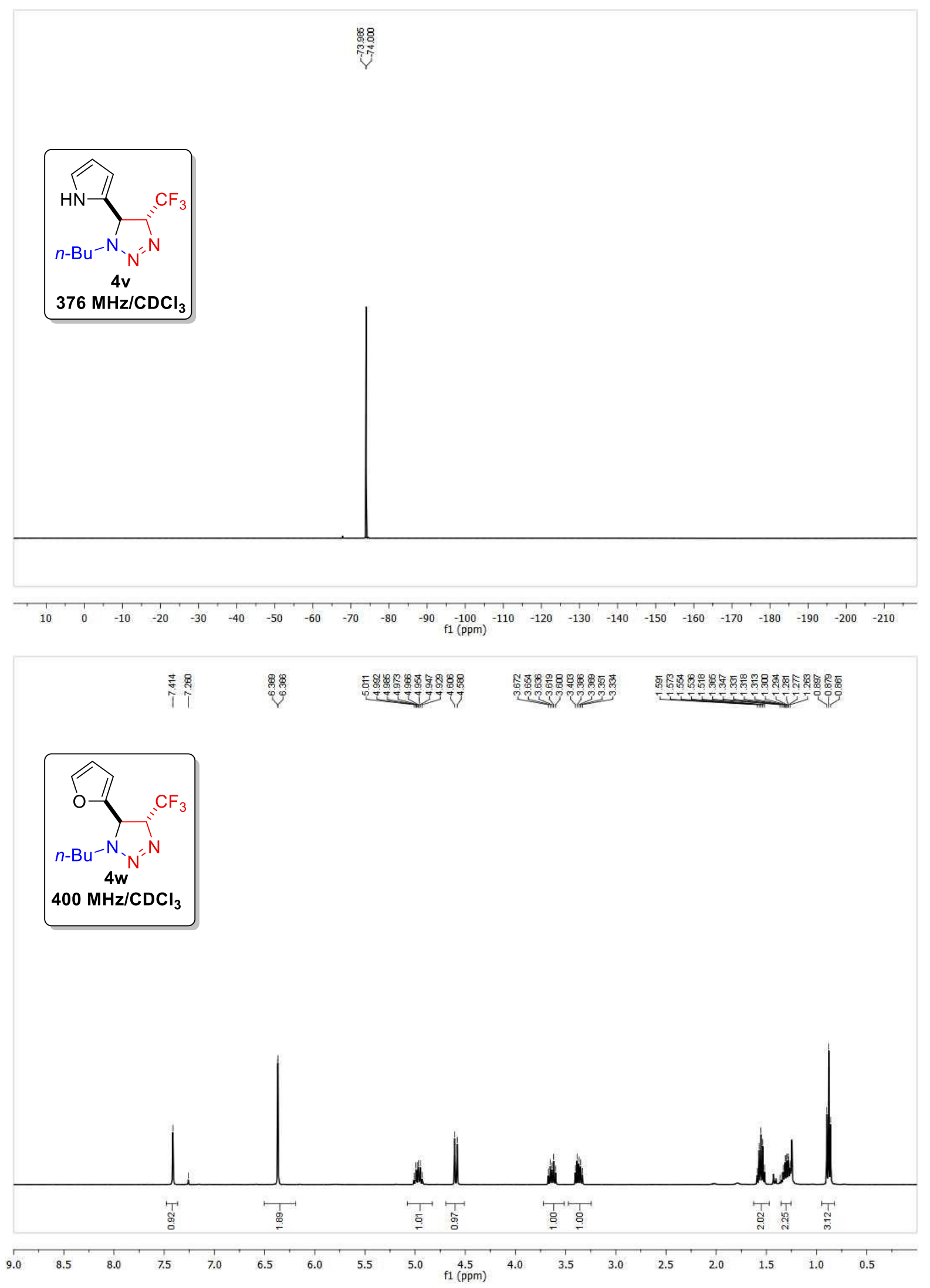



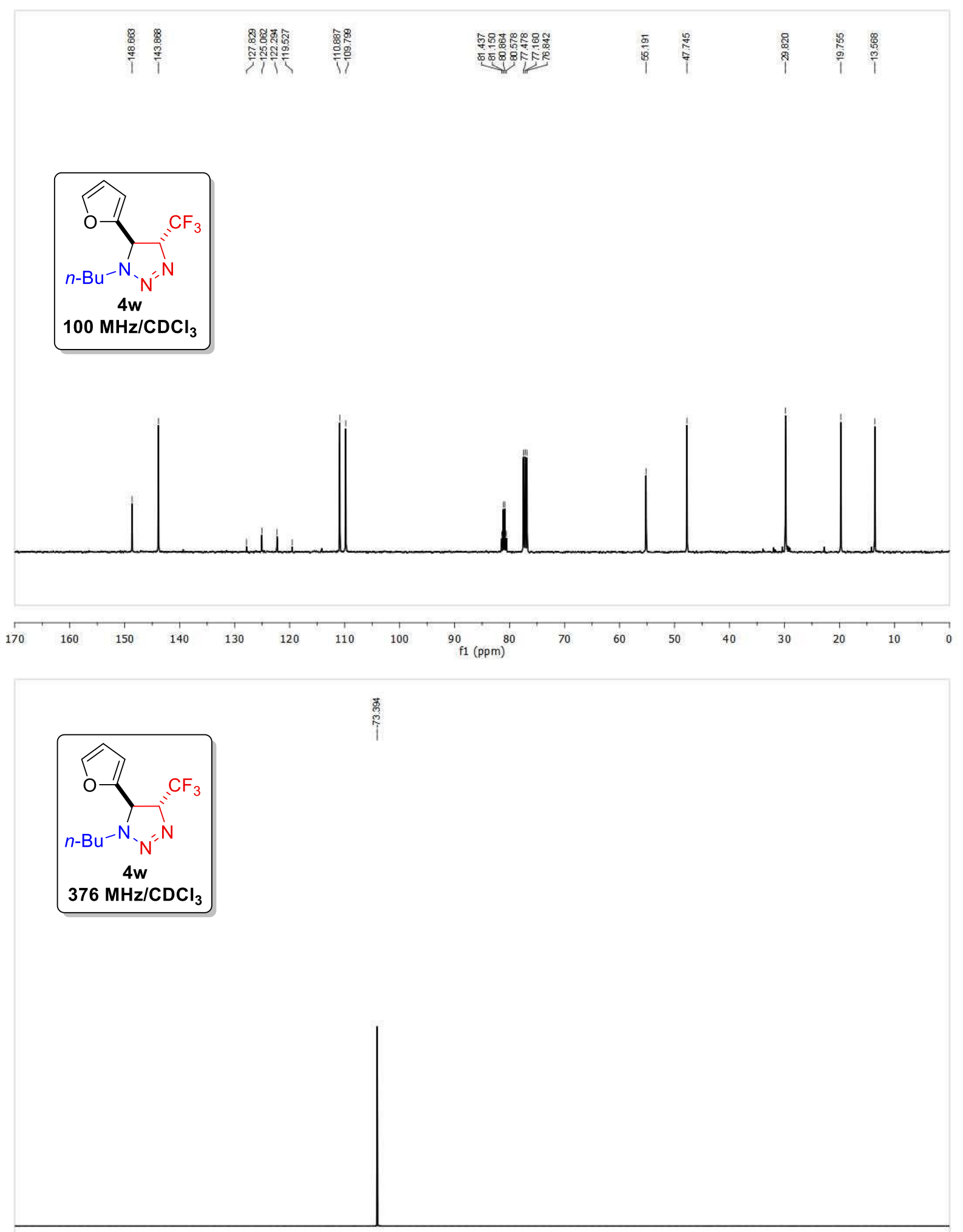

$\begin{array}{rlllllllllllllllllllllll}1 & 10 & 0 & -10 & -20 & -30 & -40 & -50 & -60 & -70 & -80 & -90 & -100 & -110 & -120 & -130 & -140 & -150 & -160 & -170 & -180 & -190 & -200 & -210\end{array}$ 

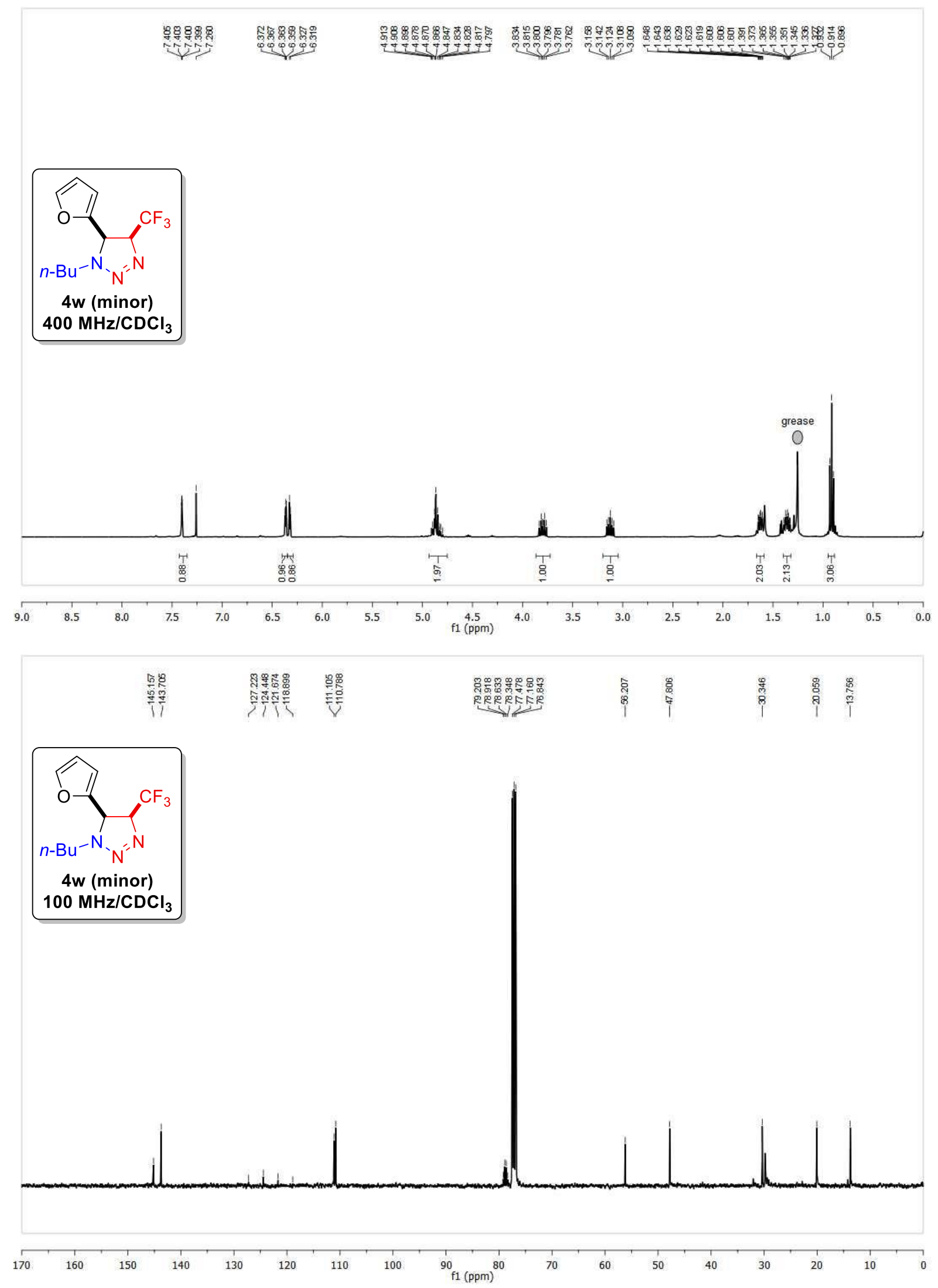


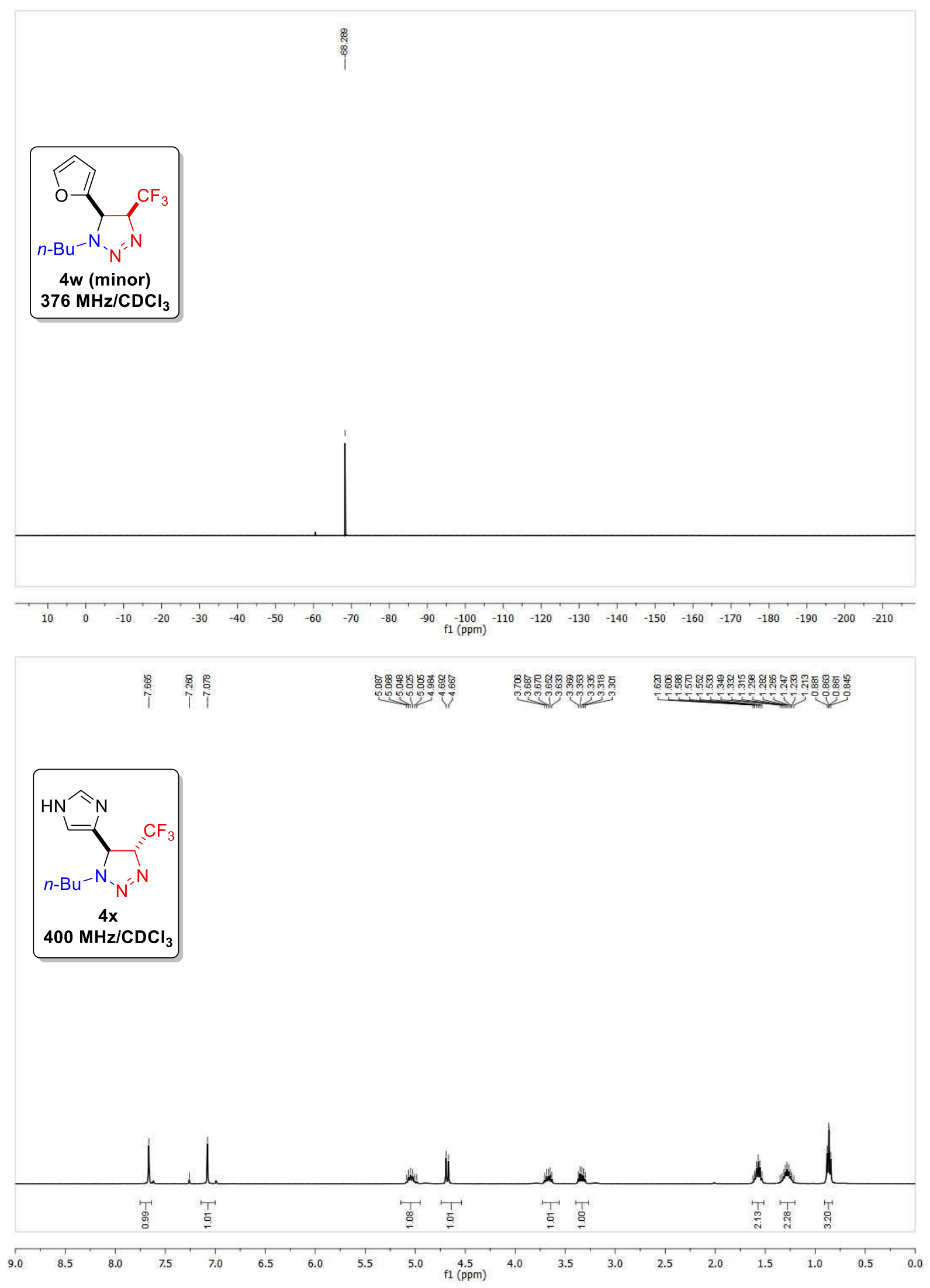



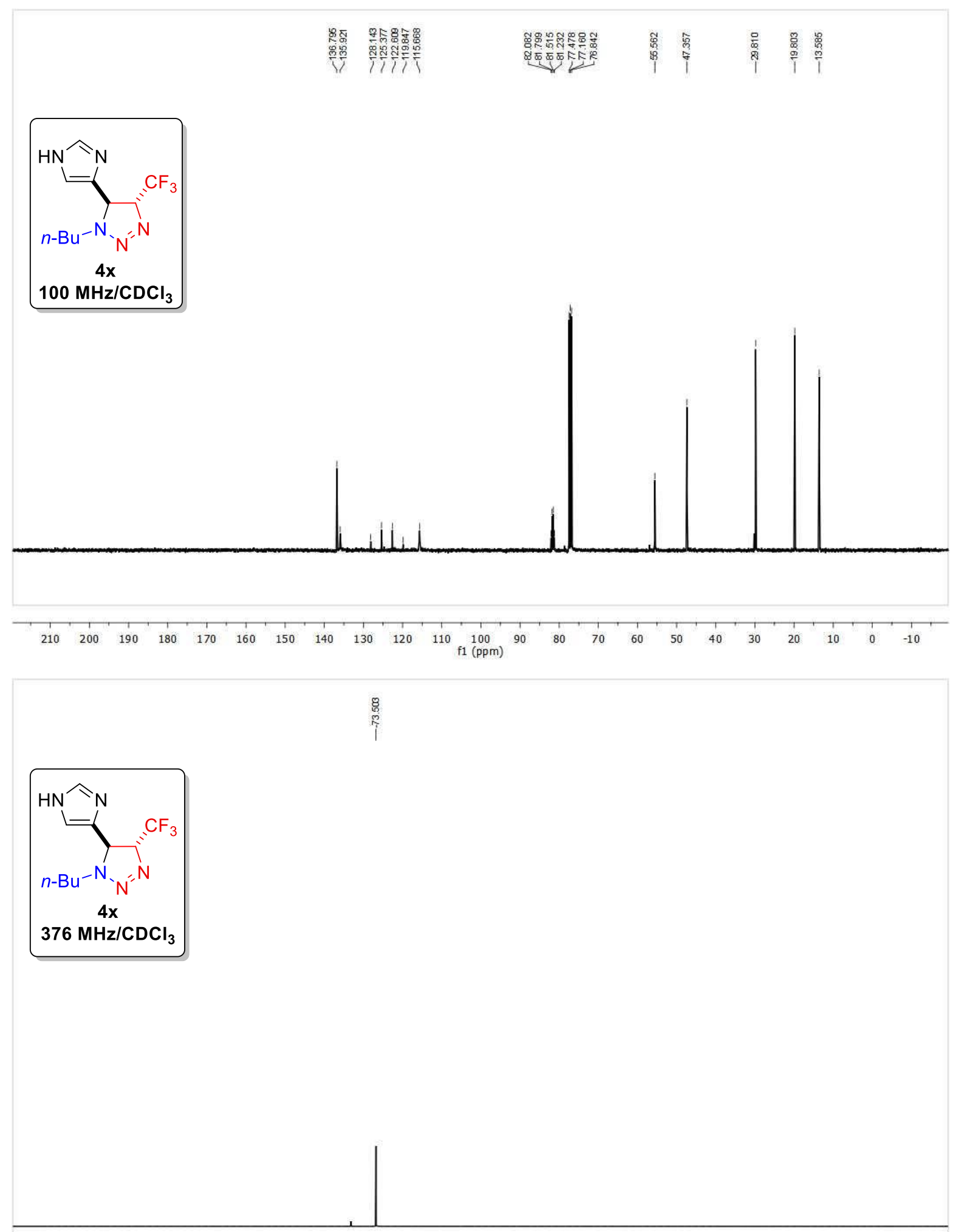

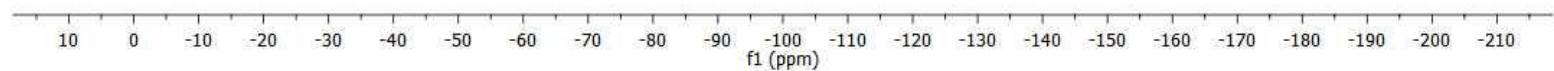



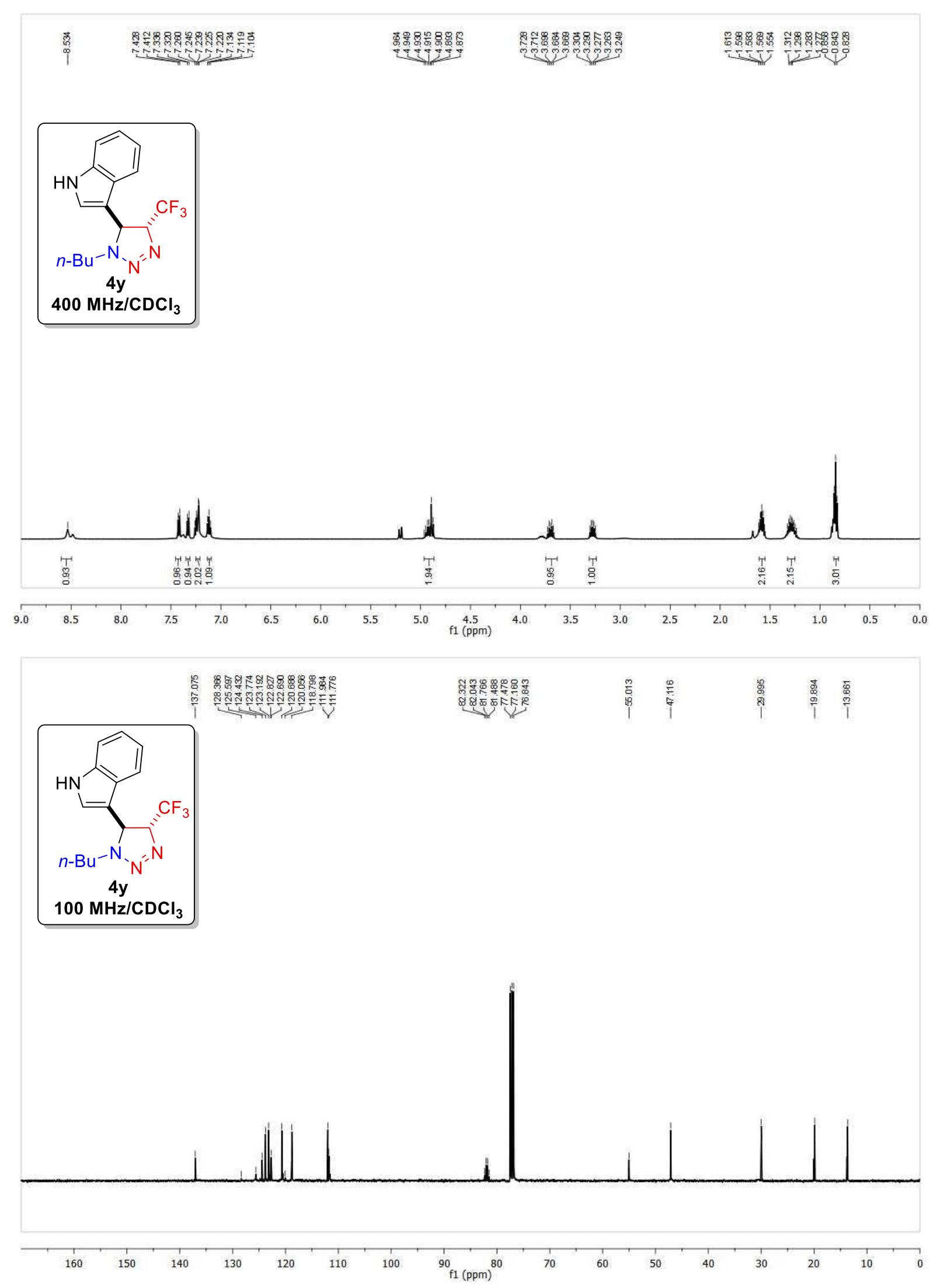


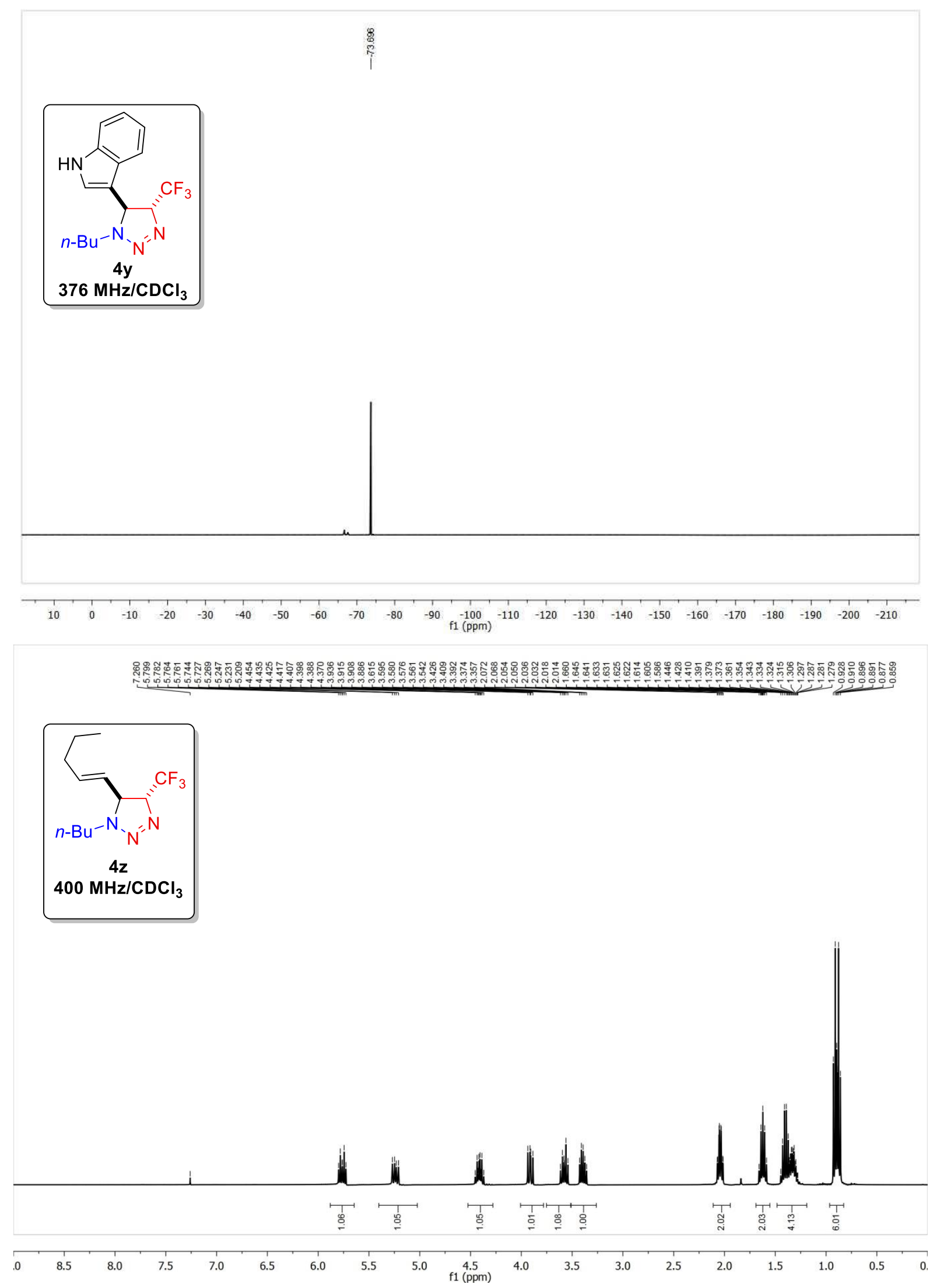




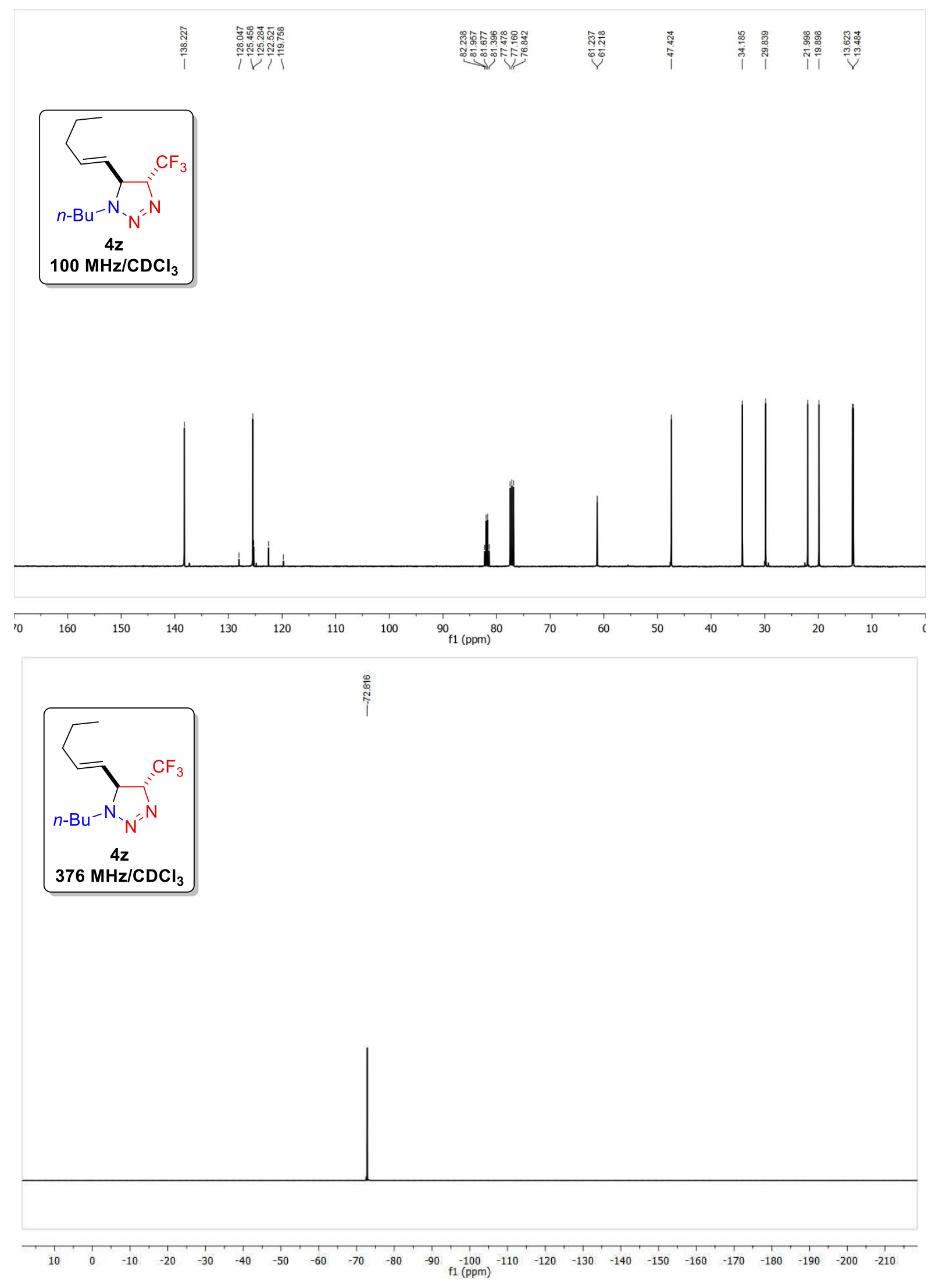



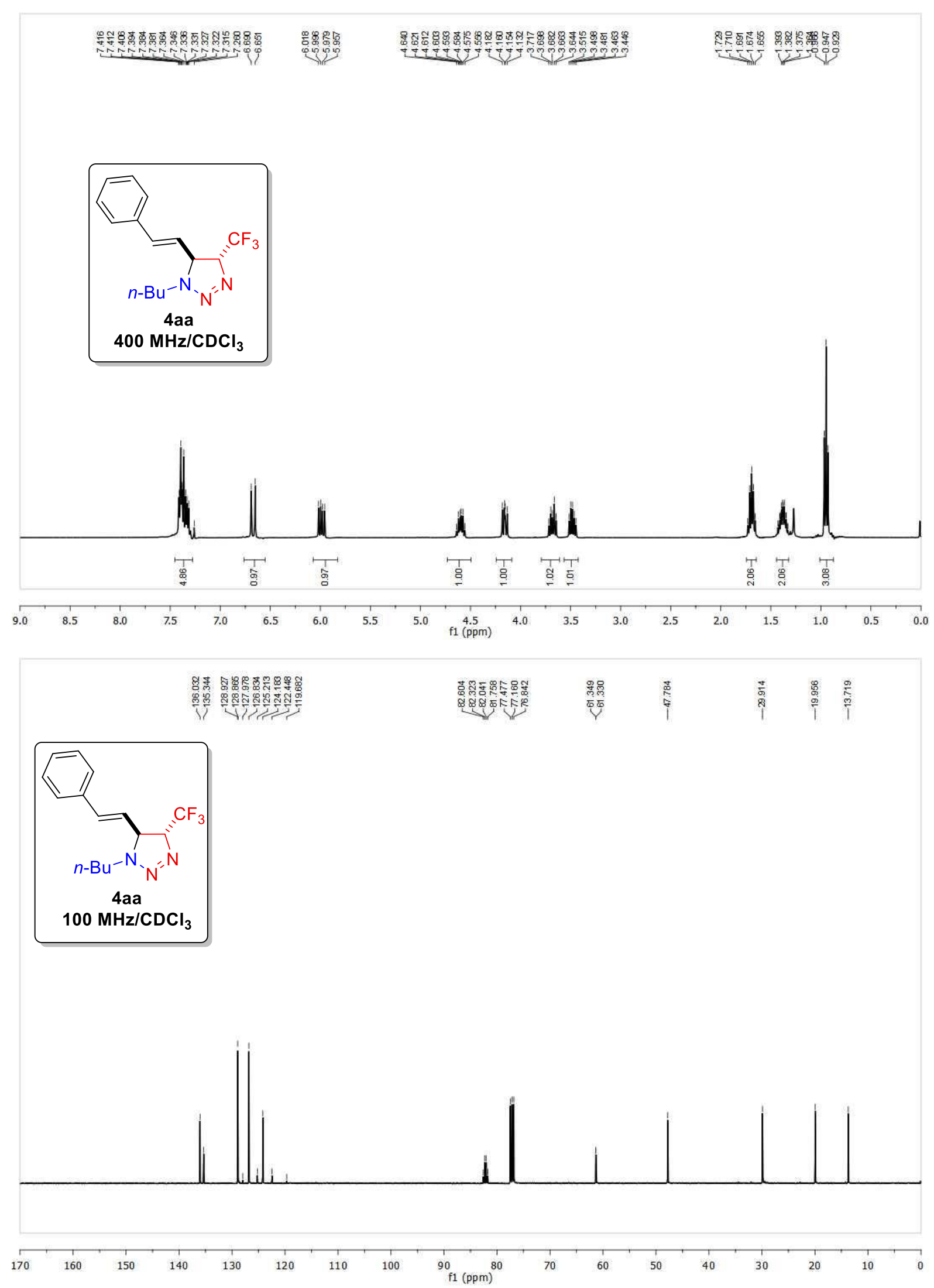

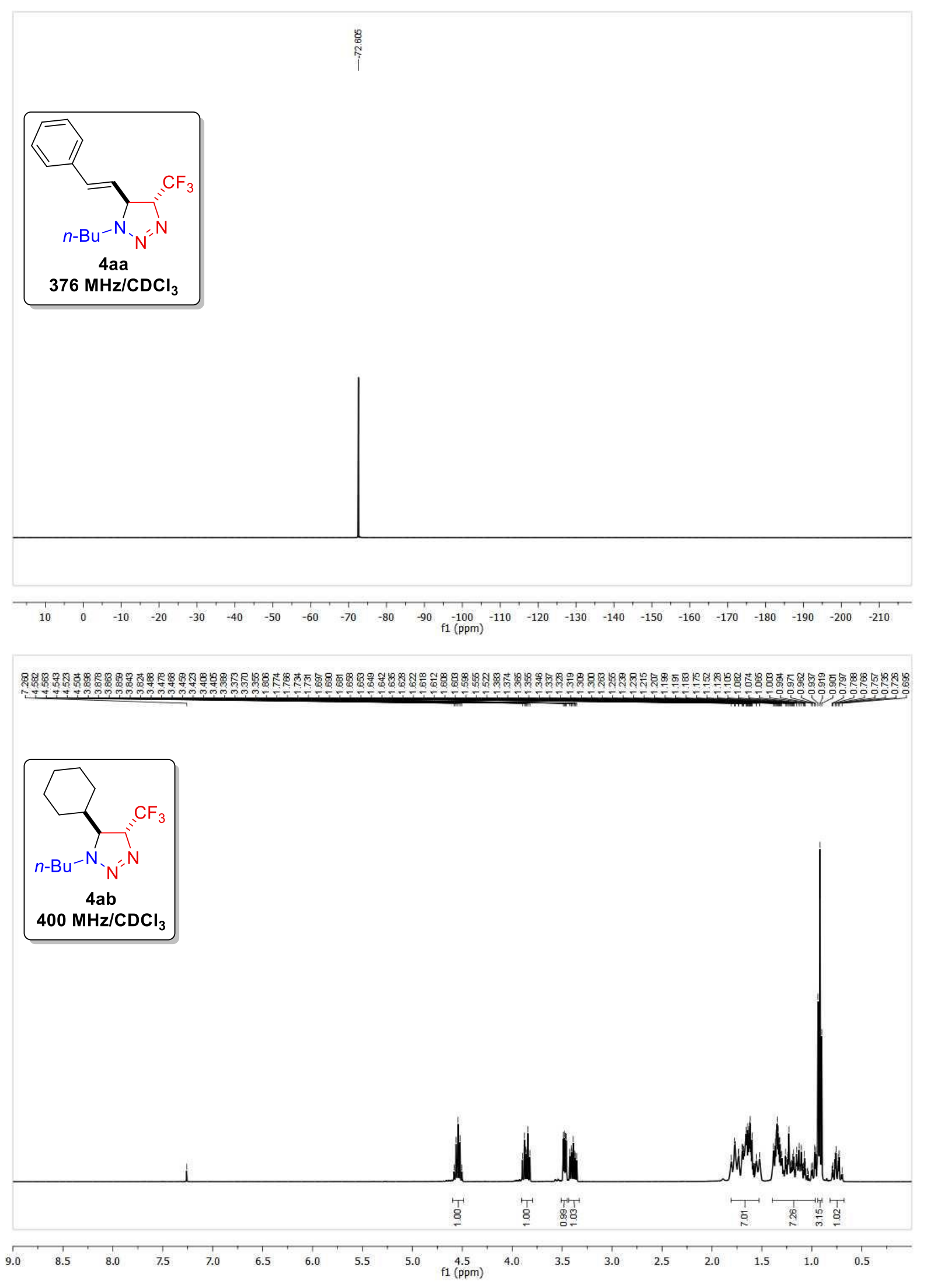


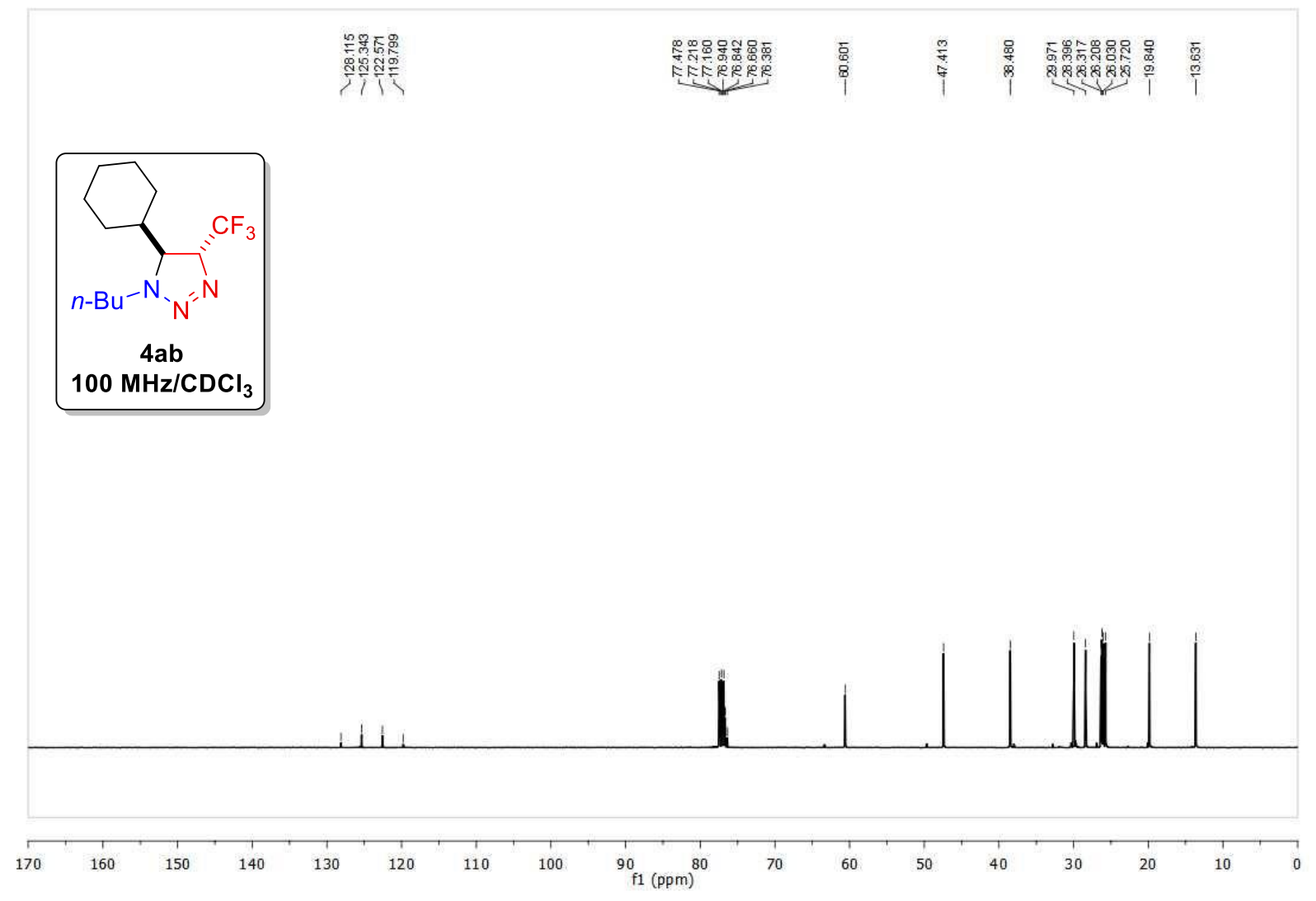

్ㅏㅁ
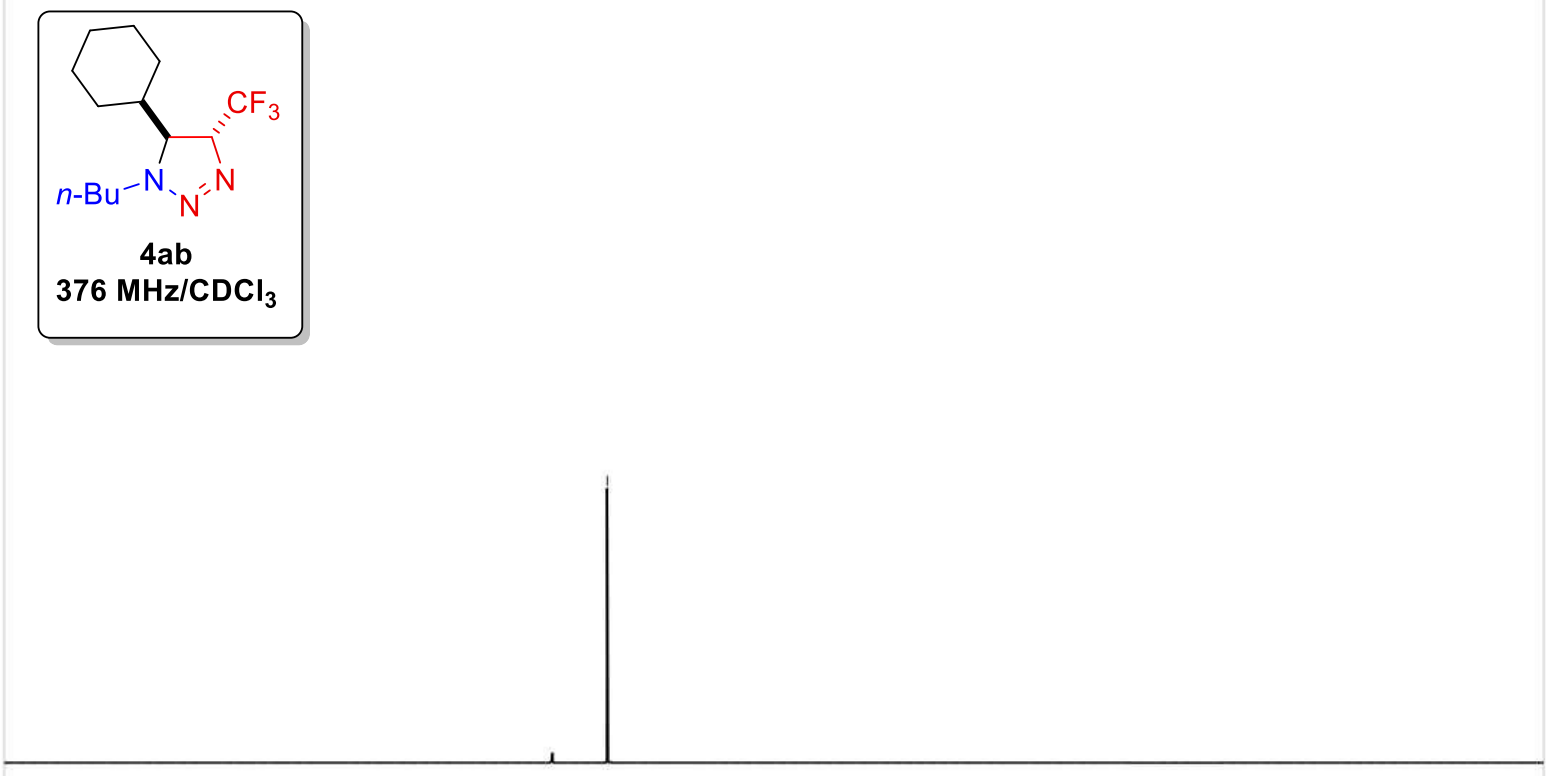

\begin{tabular}{rlllllllllllllllllllllll}
\hline & 1 & 0 & -10 & -20 & -30 & -40 & -50 & -60 & -70 & -80 & -90 & -100 & -110 & -120 & -130 & -140 & -150 & -160 & -170 & -180 & -190 & -200 & -210
\end{tabular} 

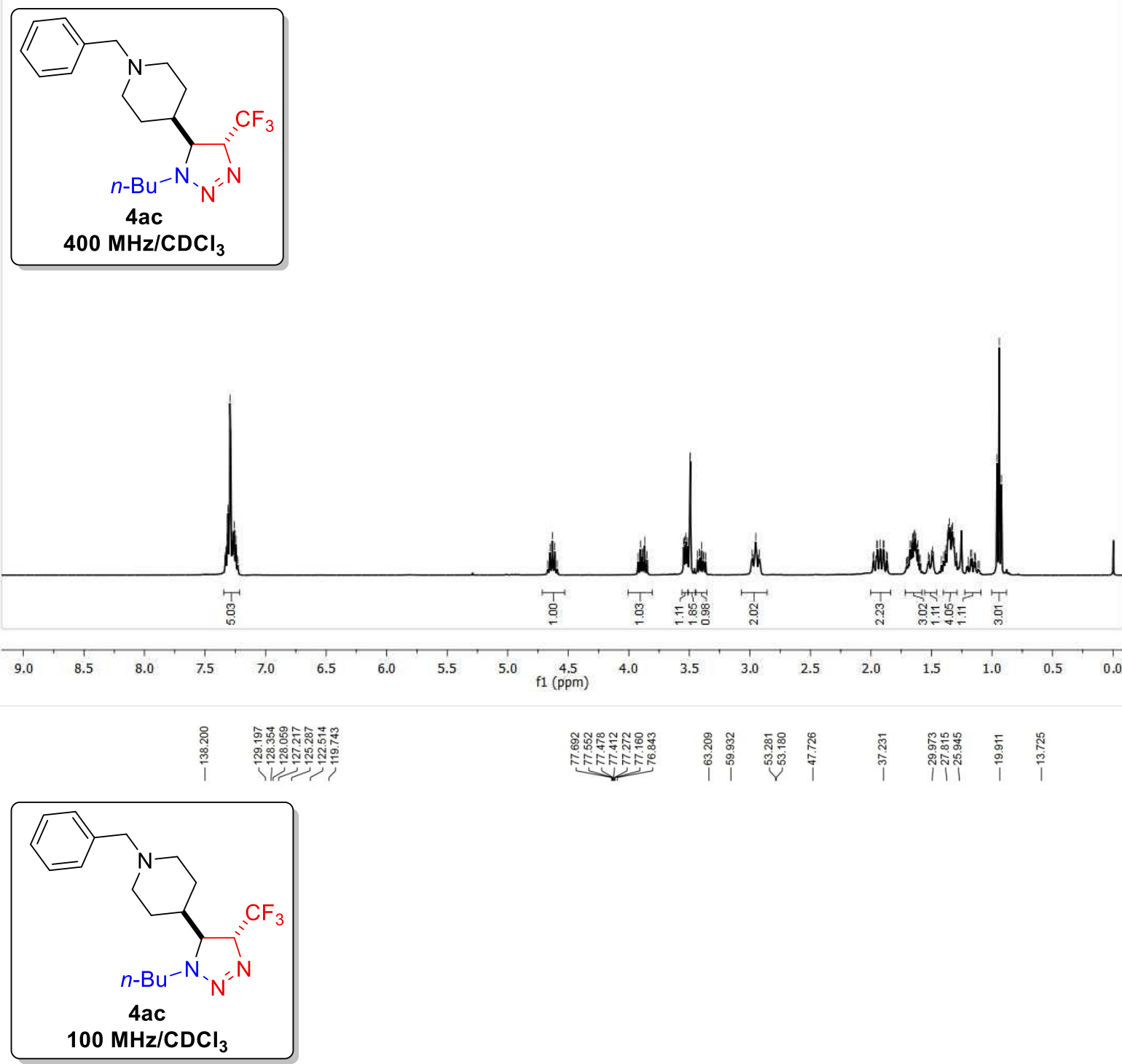

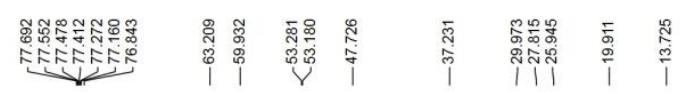

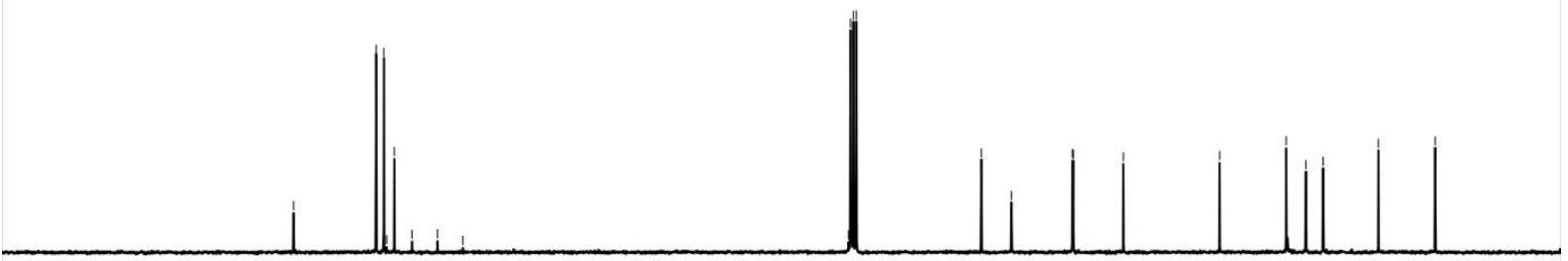

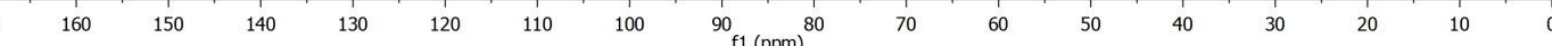




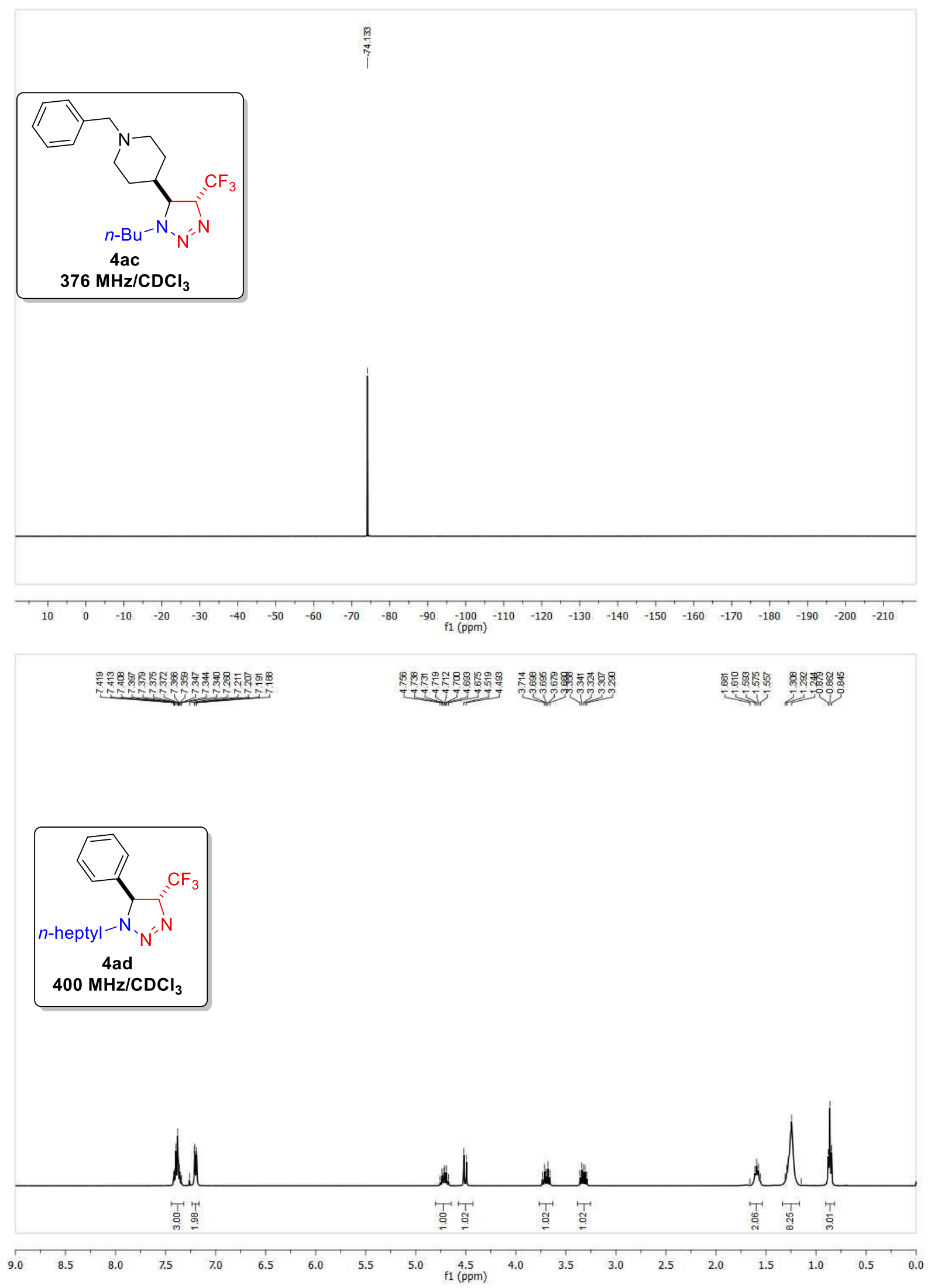



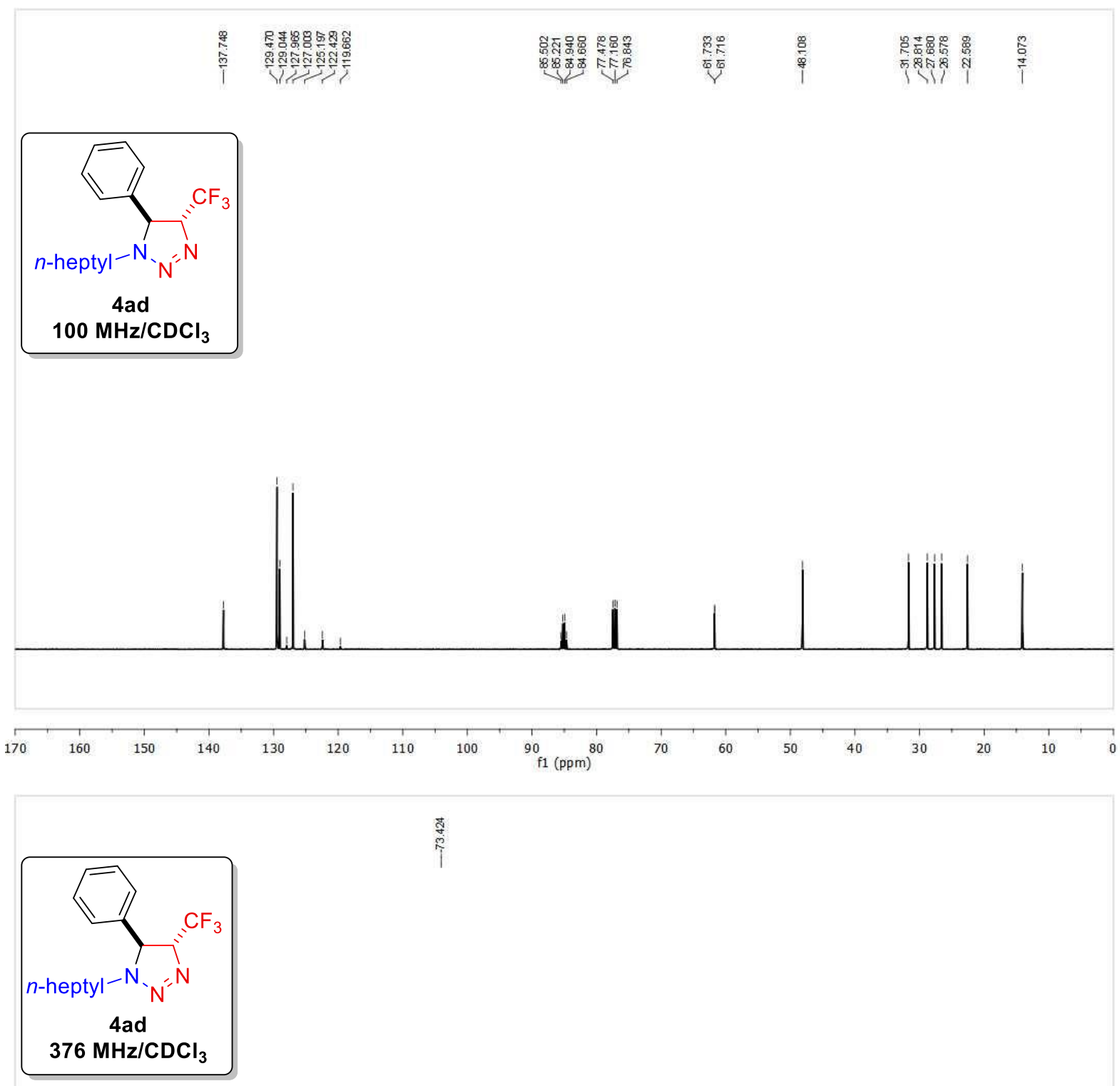

गู

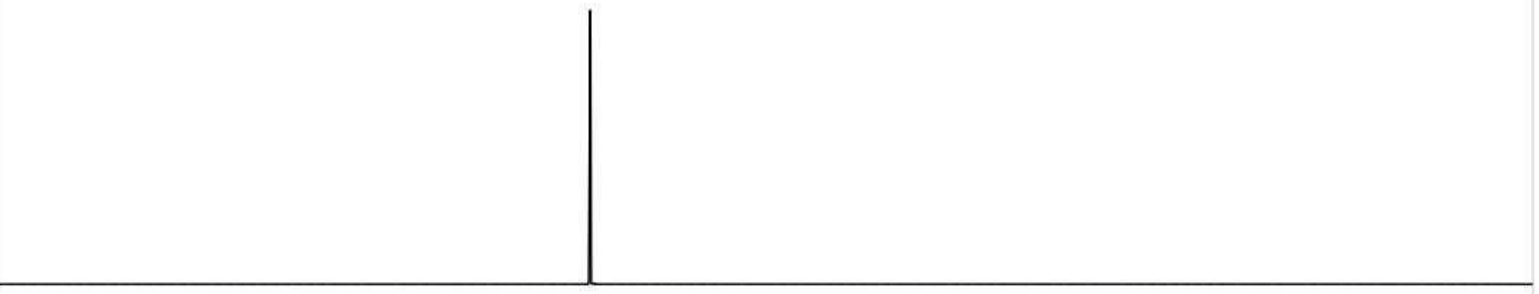

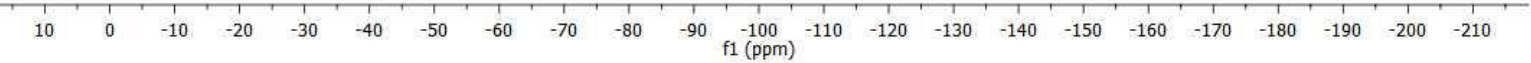




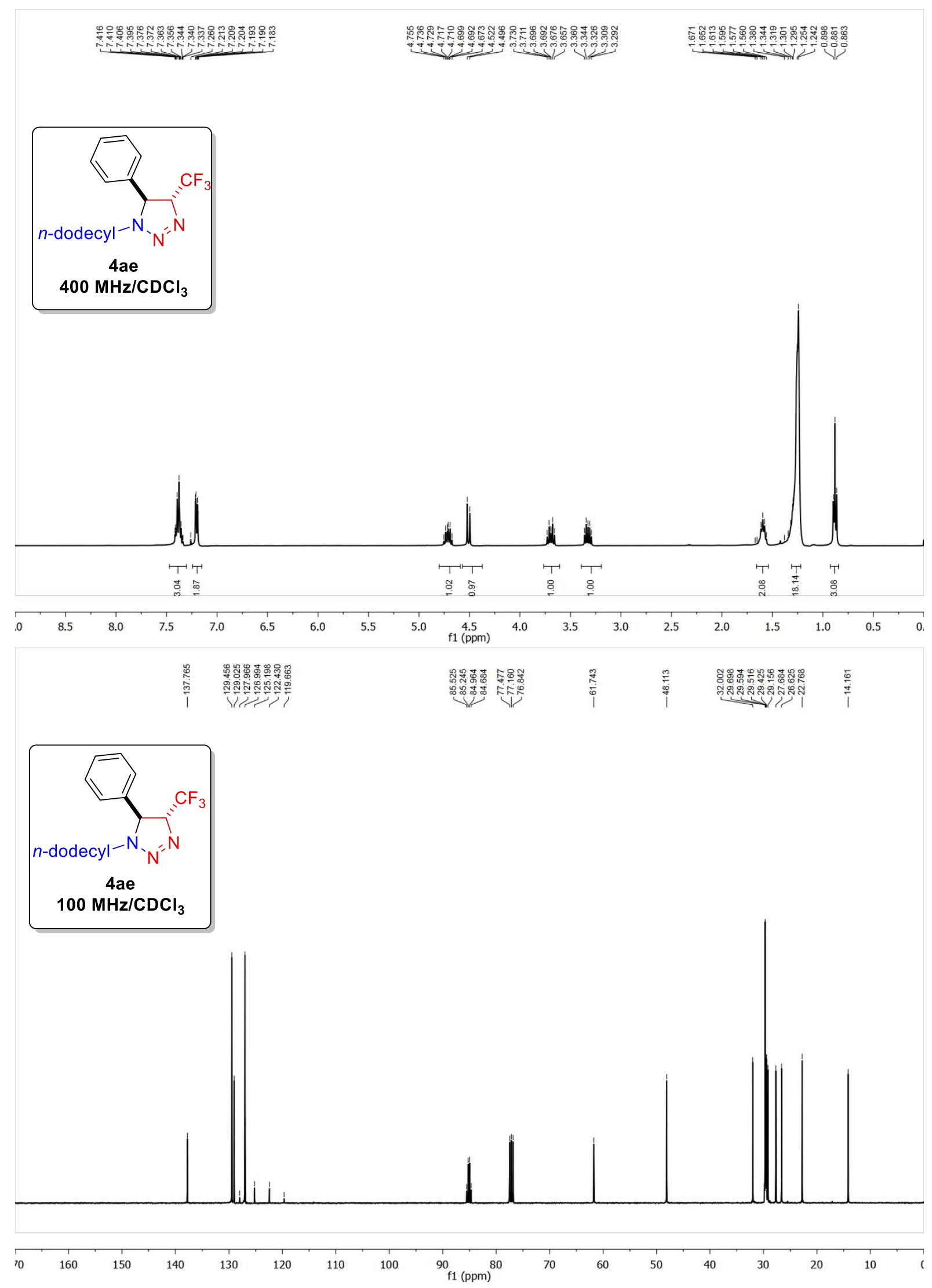




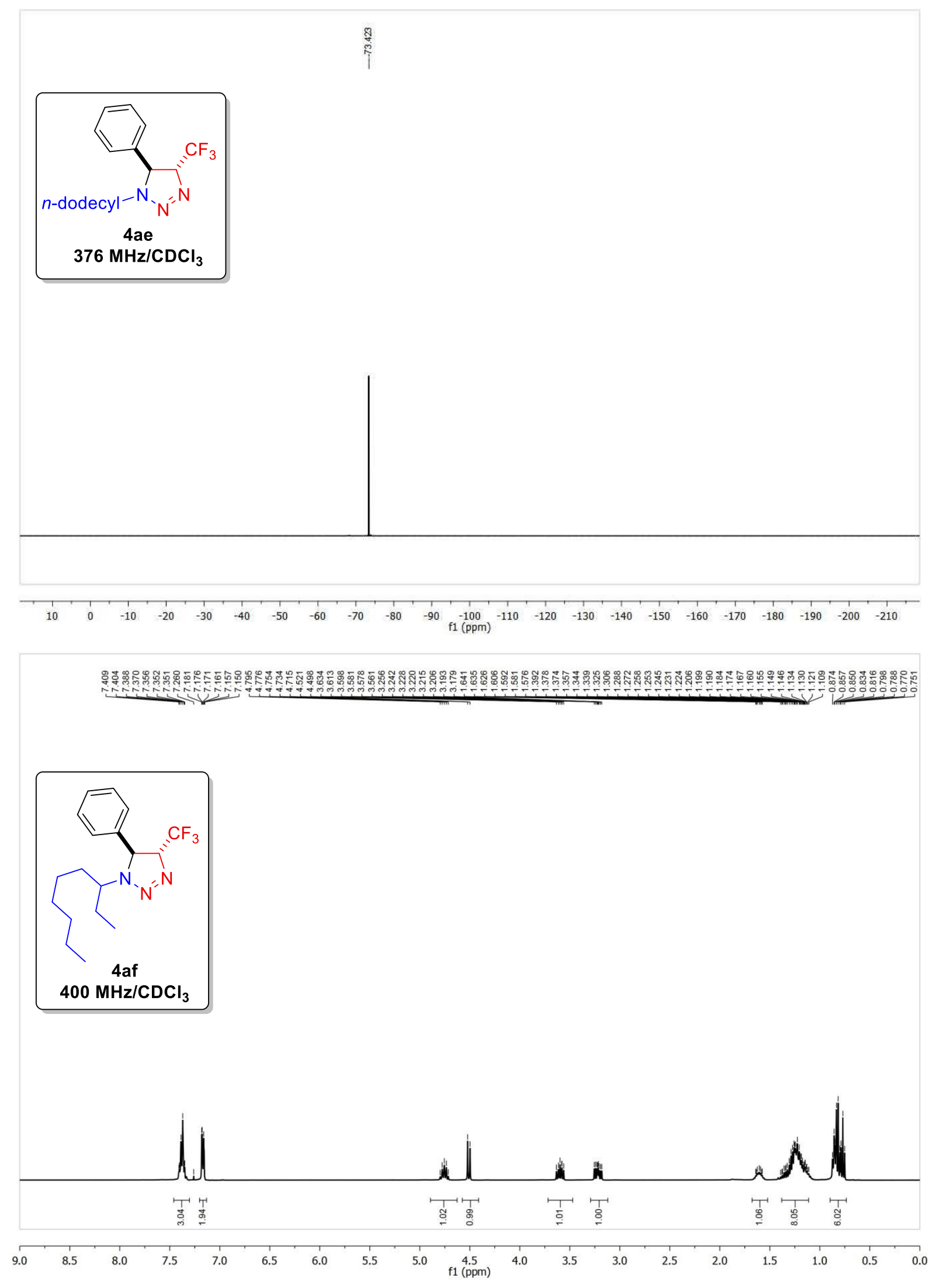



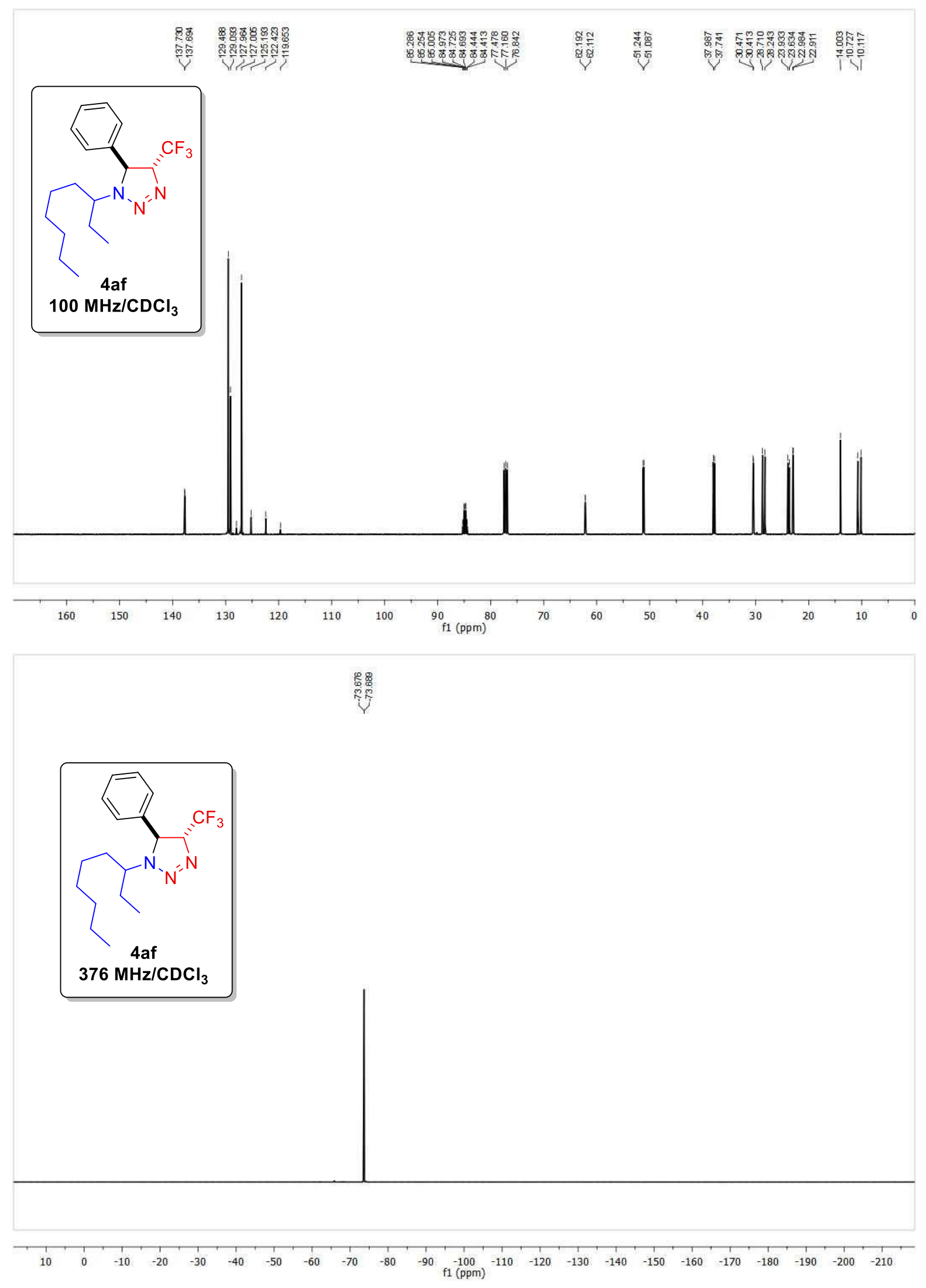

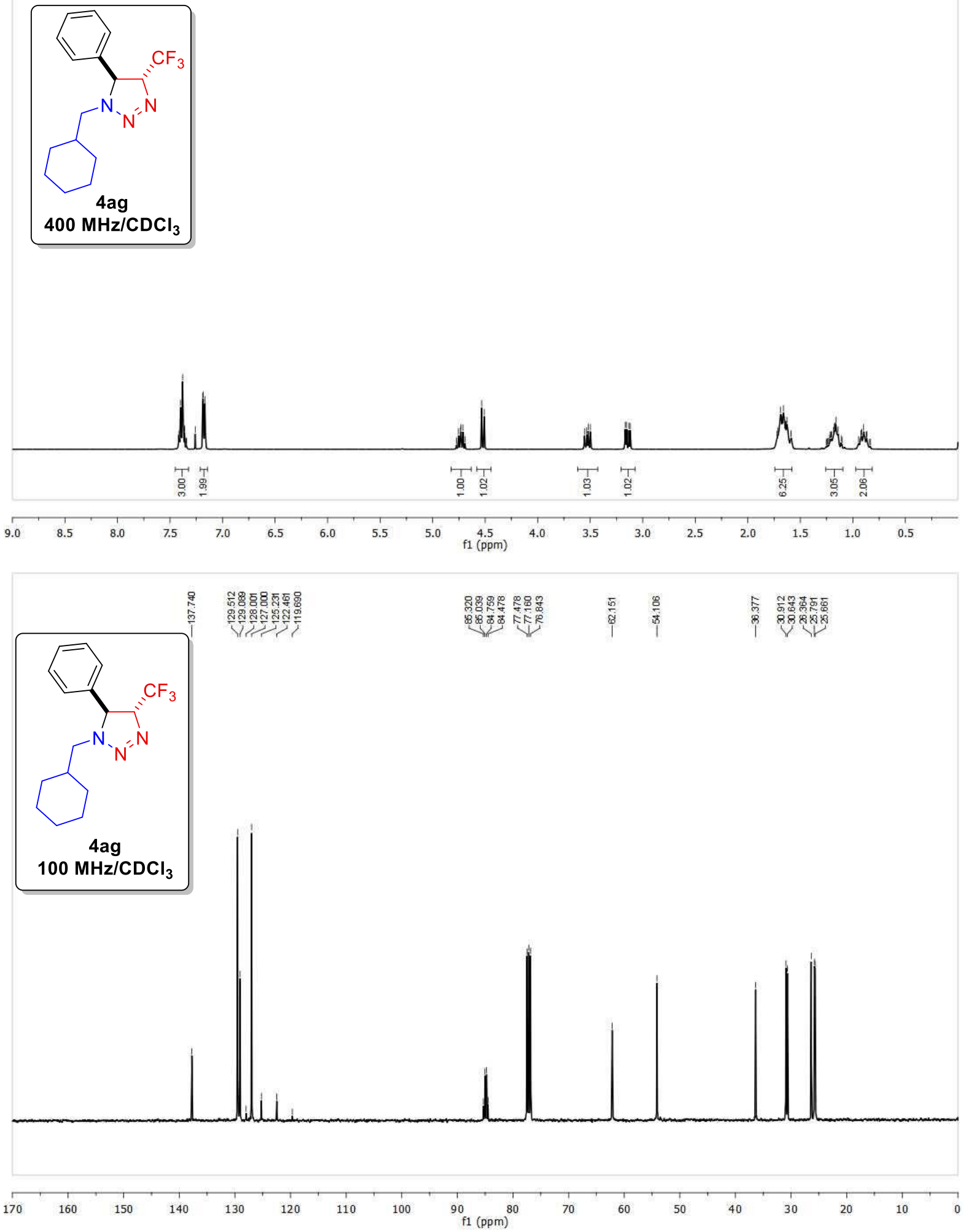


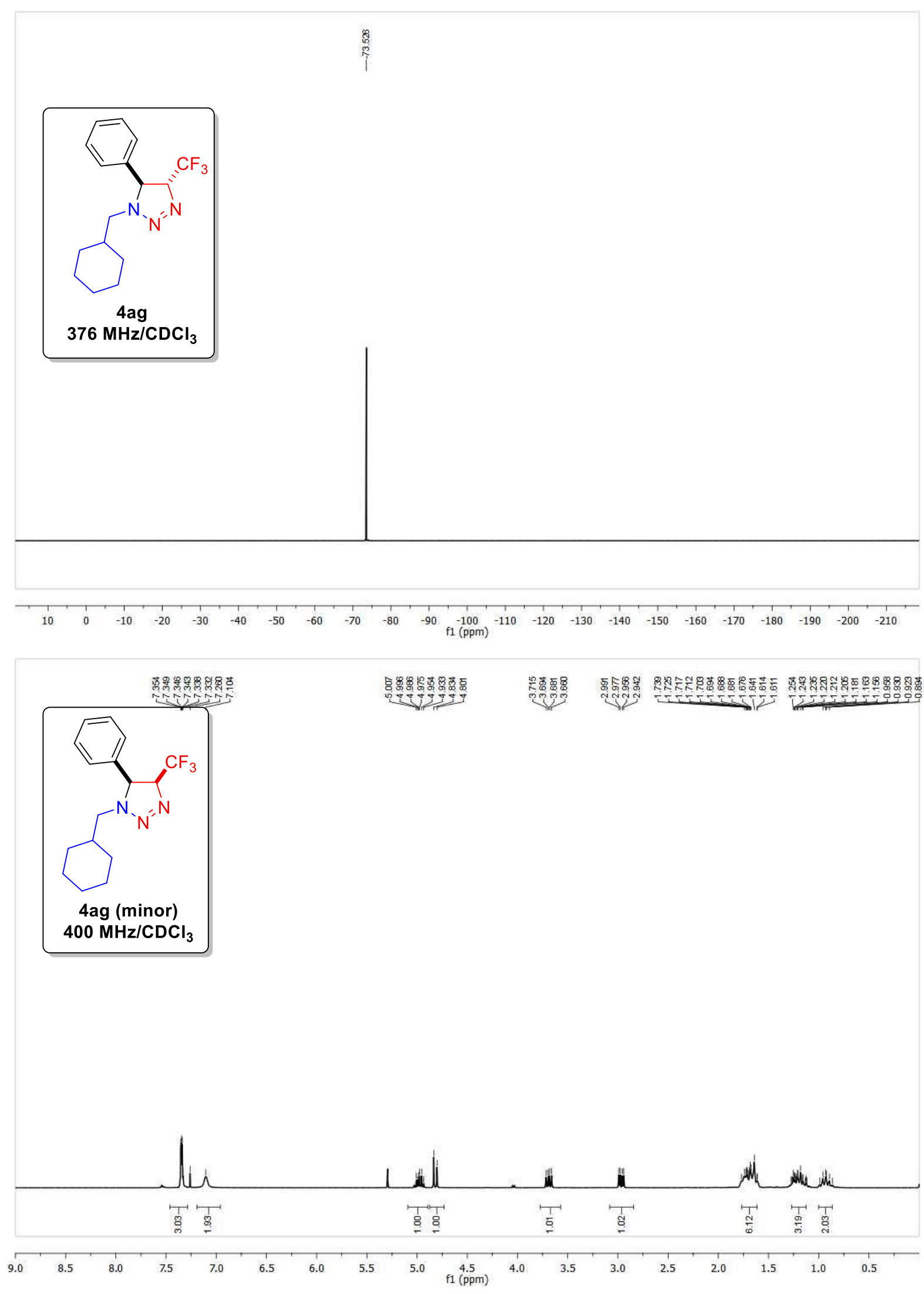



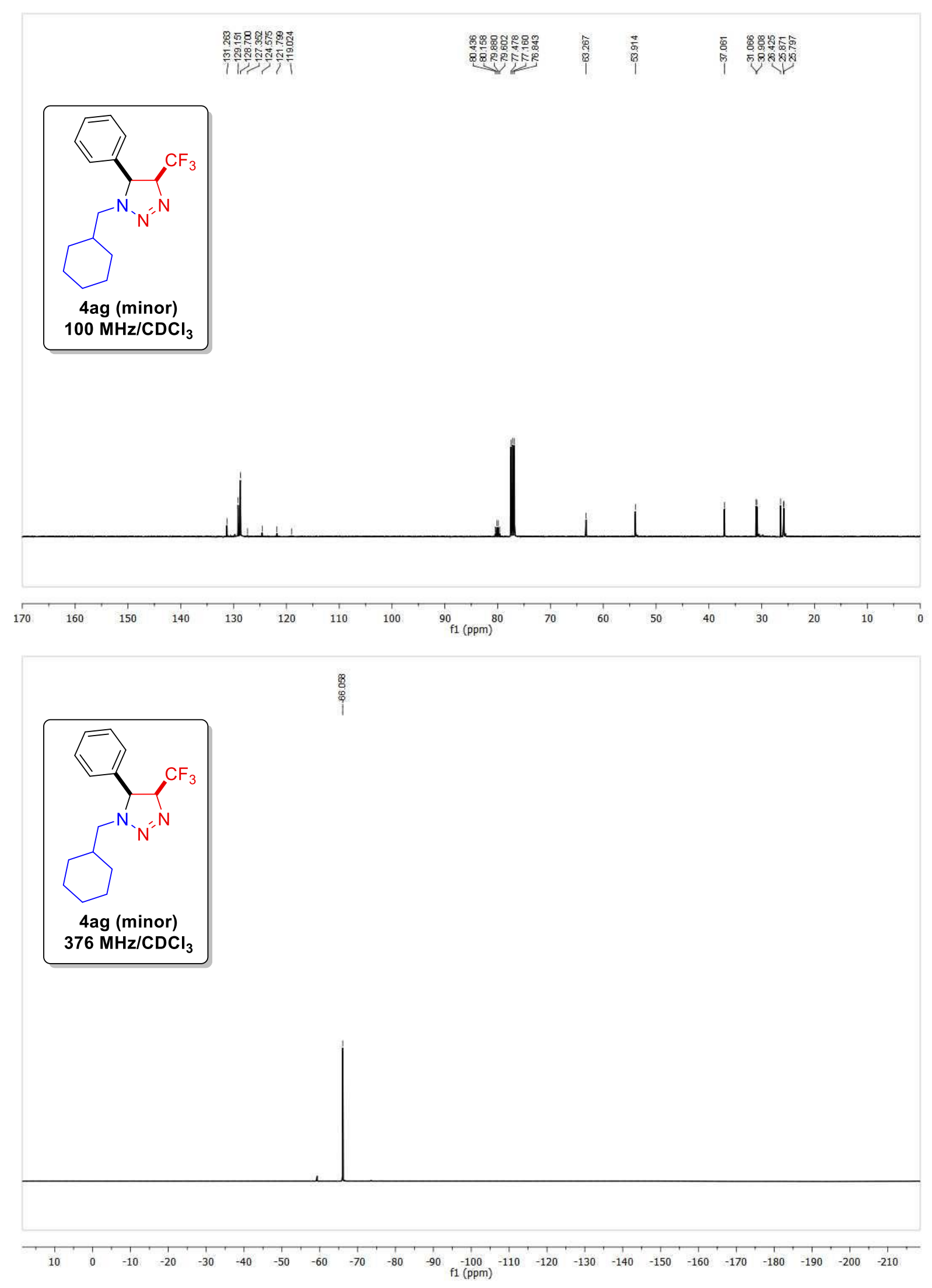


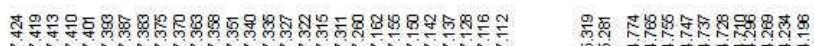

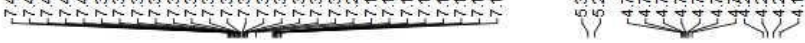
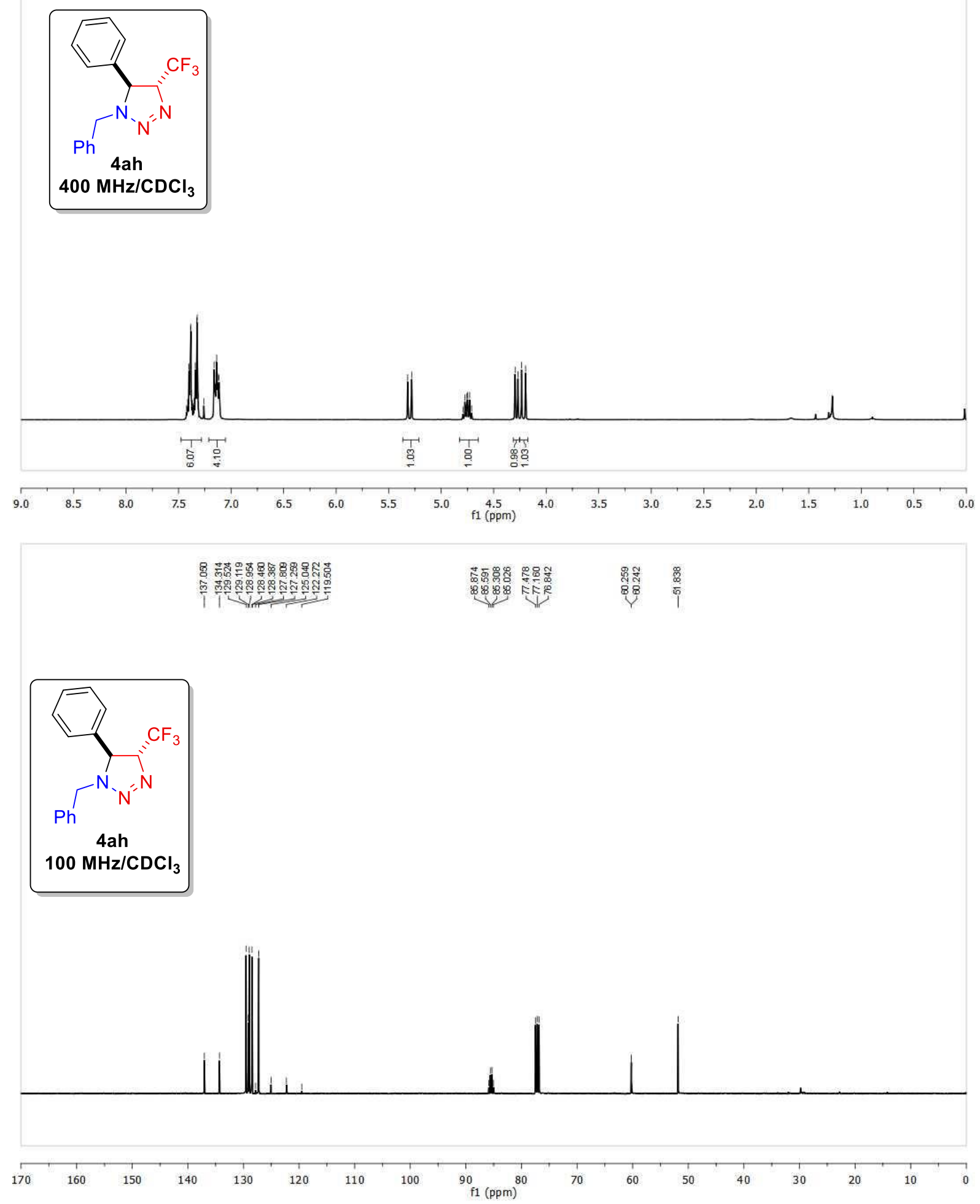


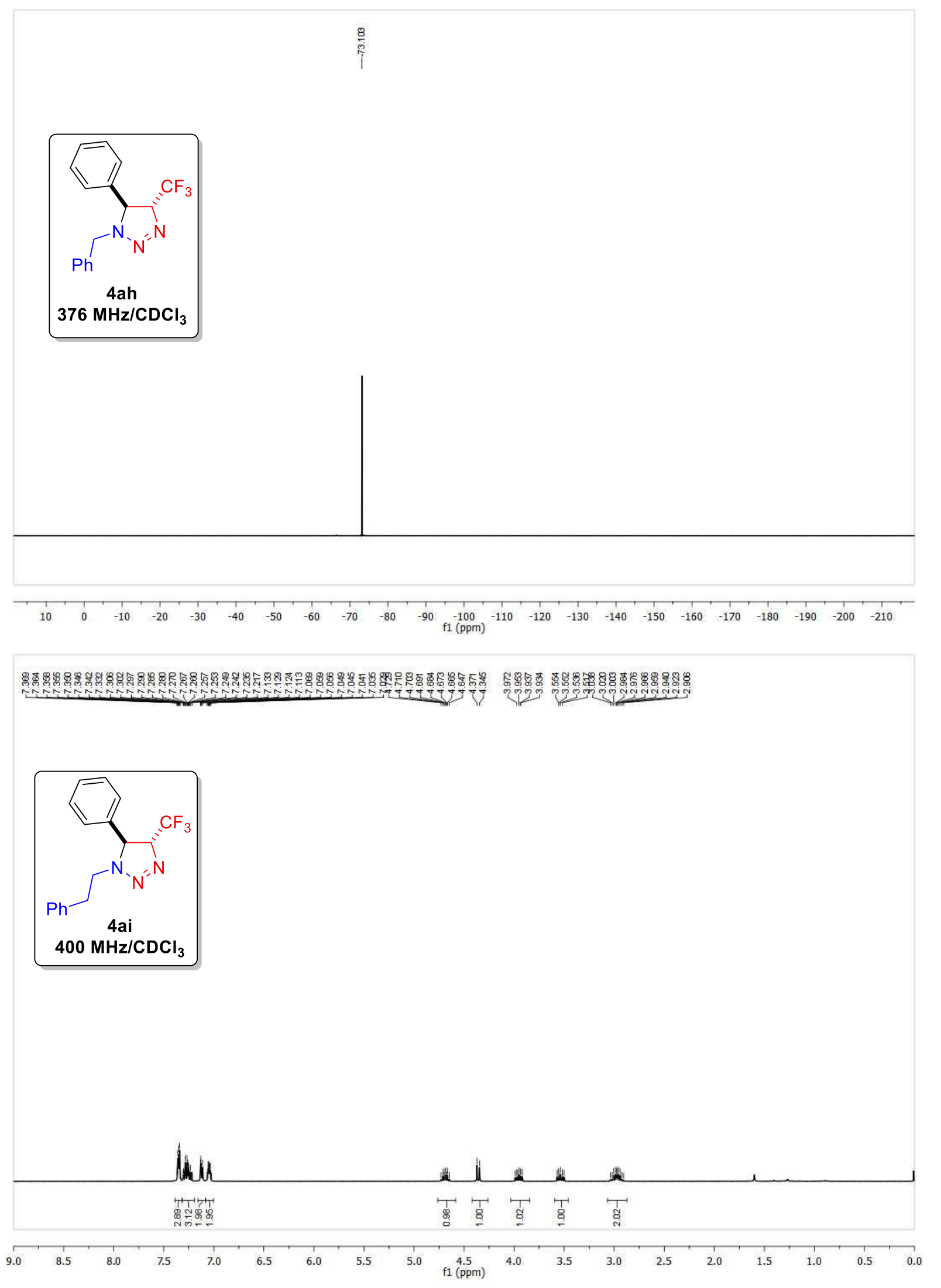



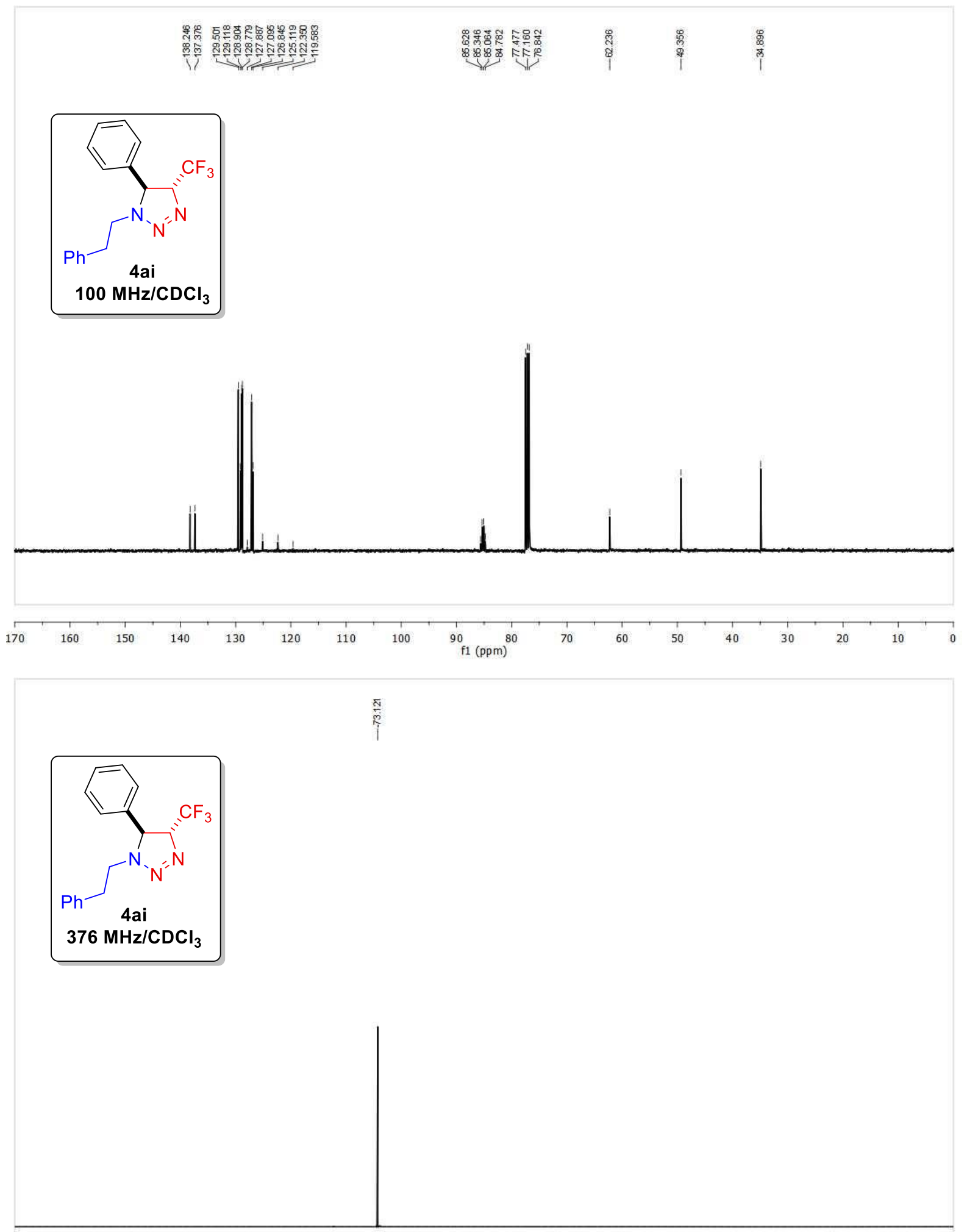

$\begin{array}{rlllllllllllllllllllllll}1 & 10 & 0 & -10 & -20 & -30 & -40 & -50 & -60 & -70 & -80 & -90 & -100 & -110 & -120 & -130 & -140 & -150 & -160 & -170 & -180 & -190 & -200 & -210\end{array}$ 


\section{8:}
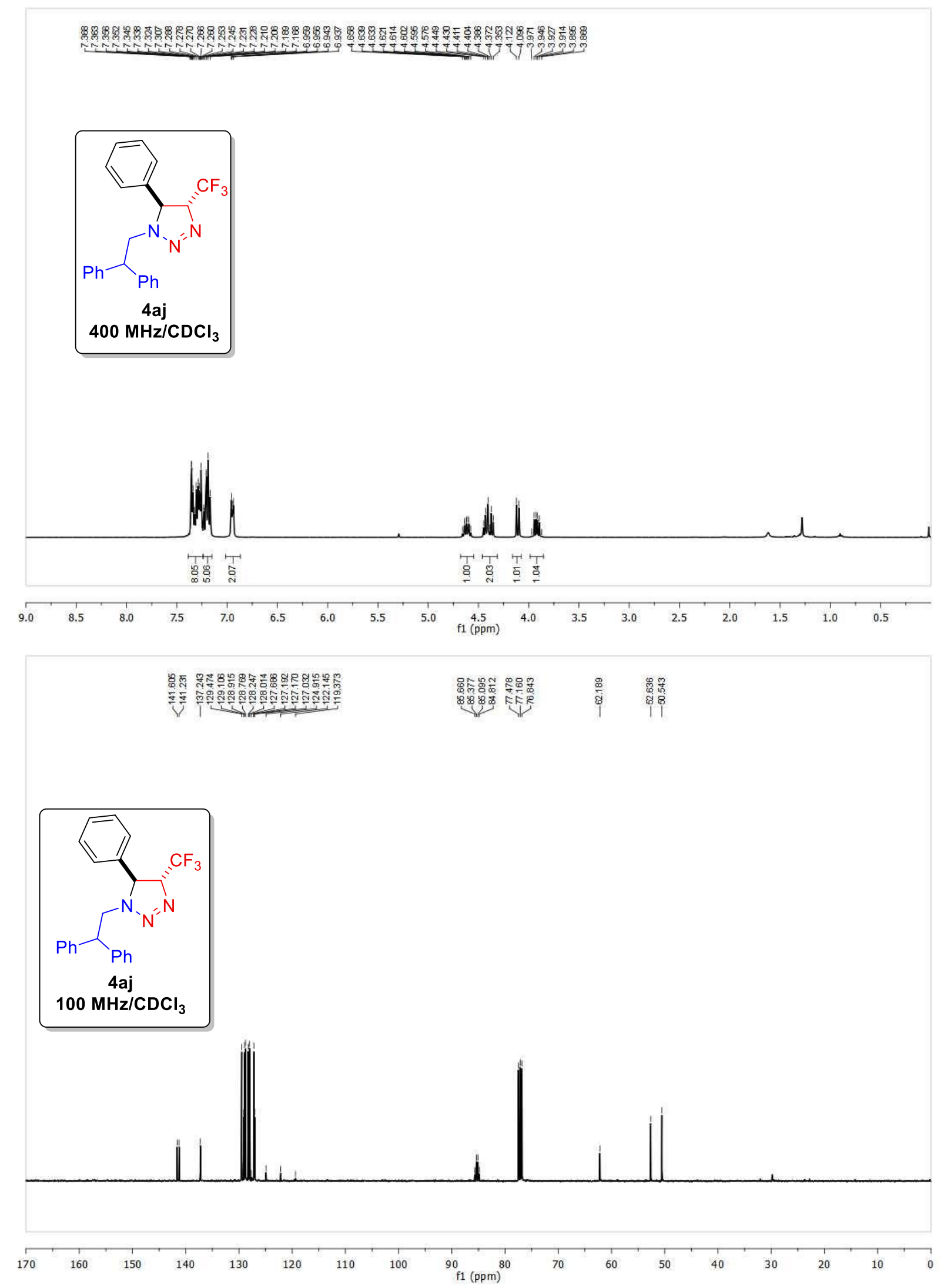


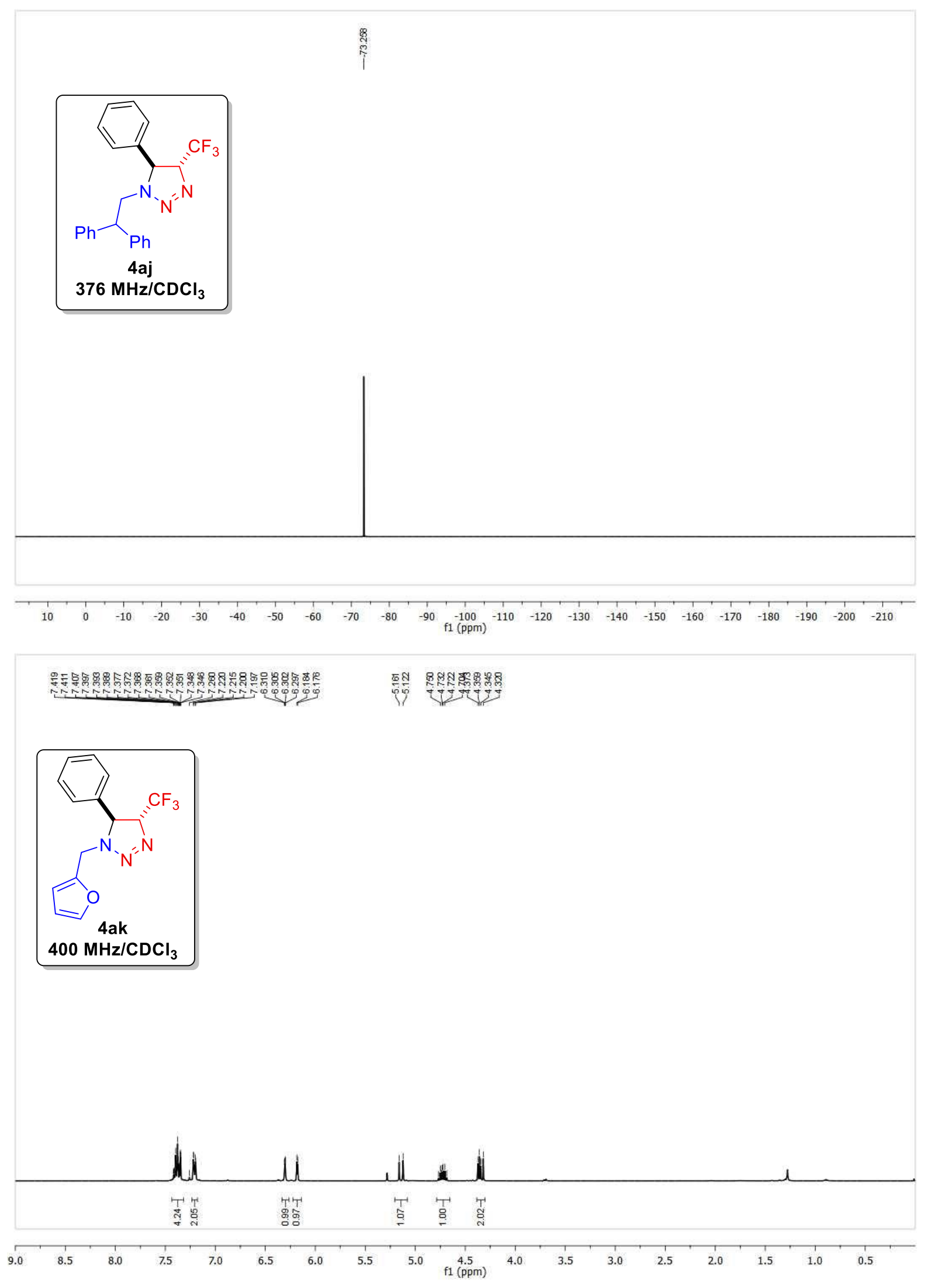



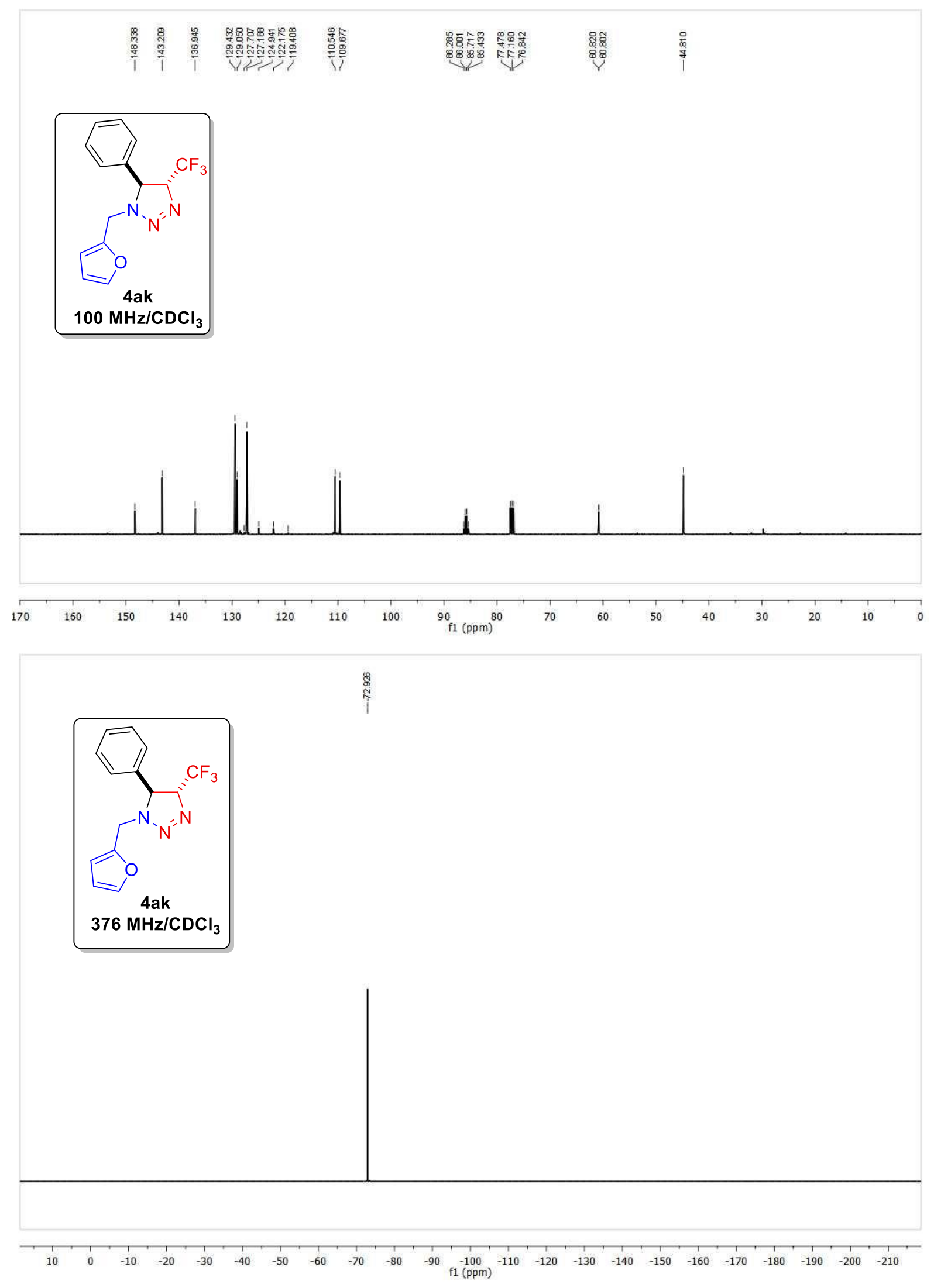

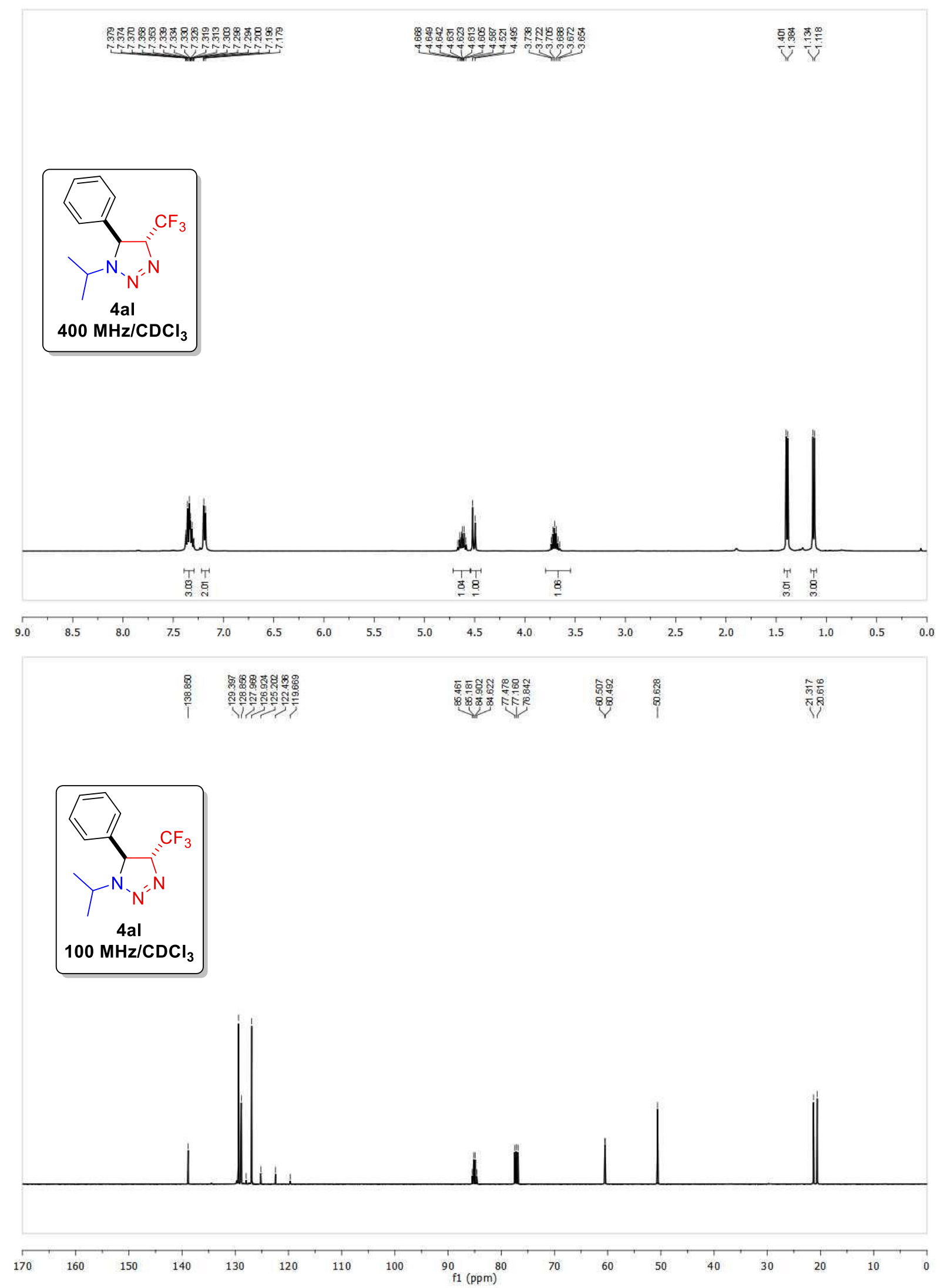


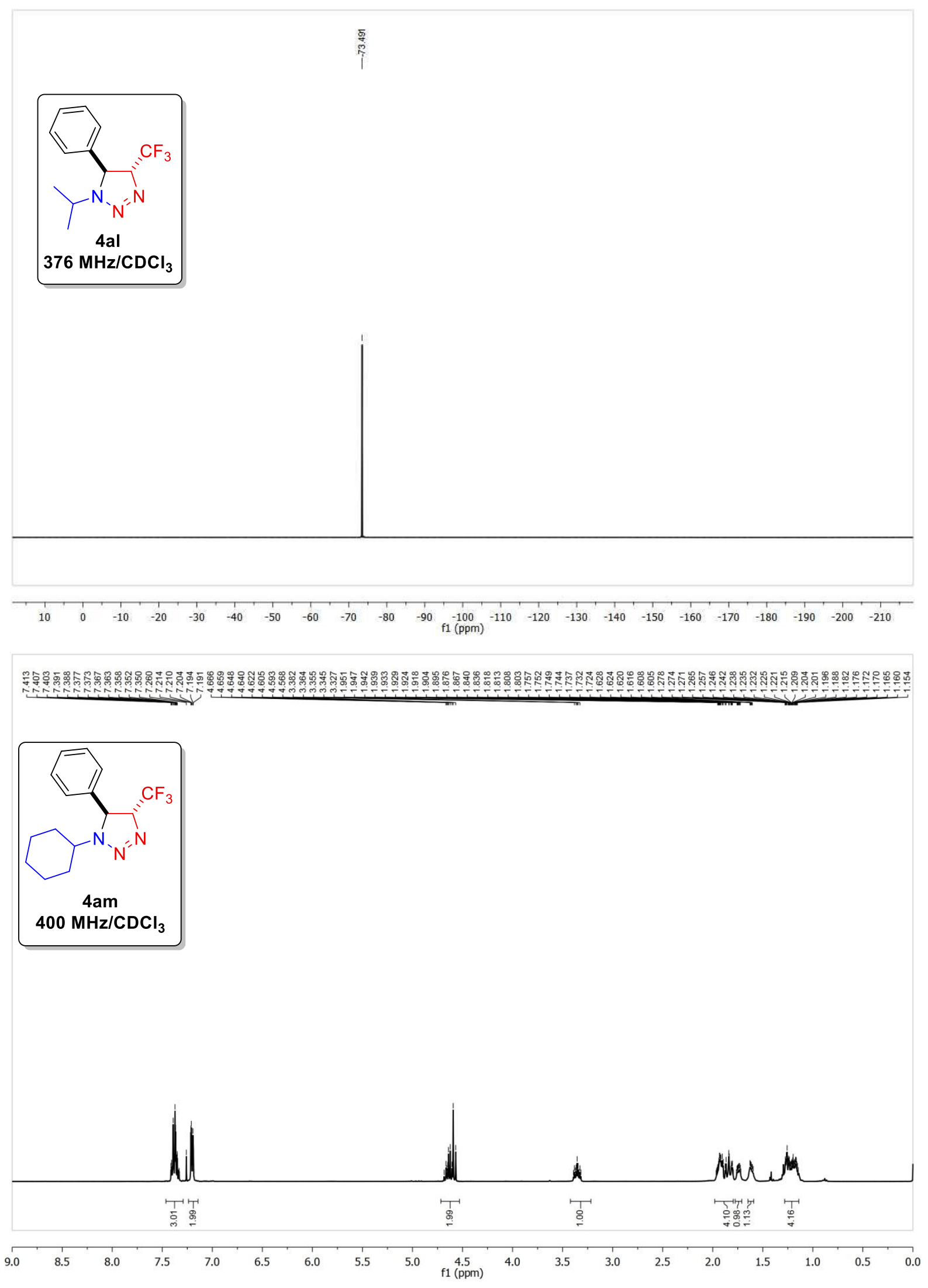



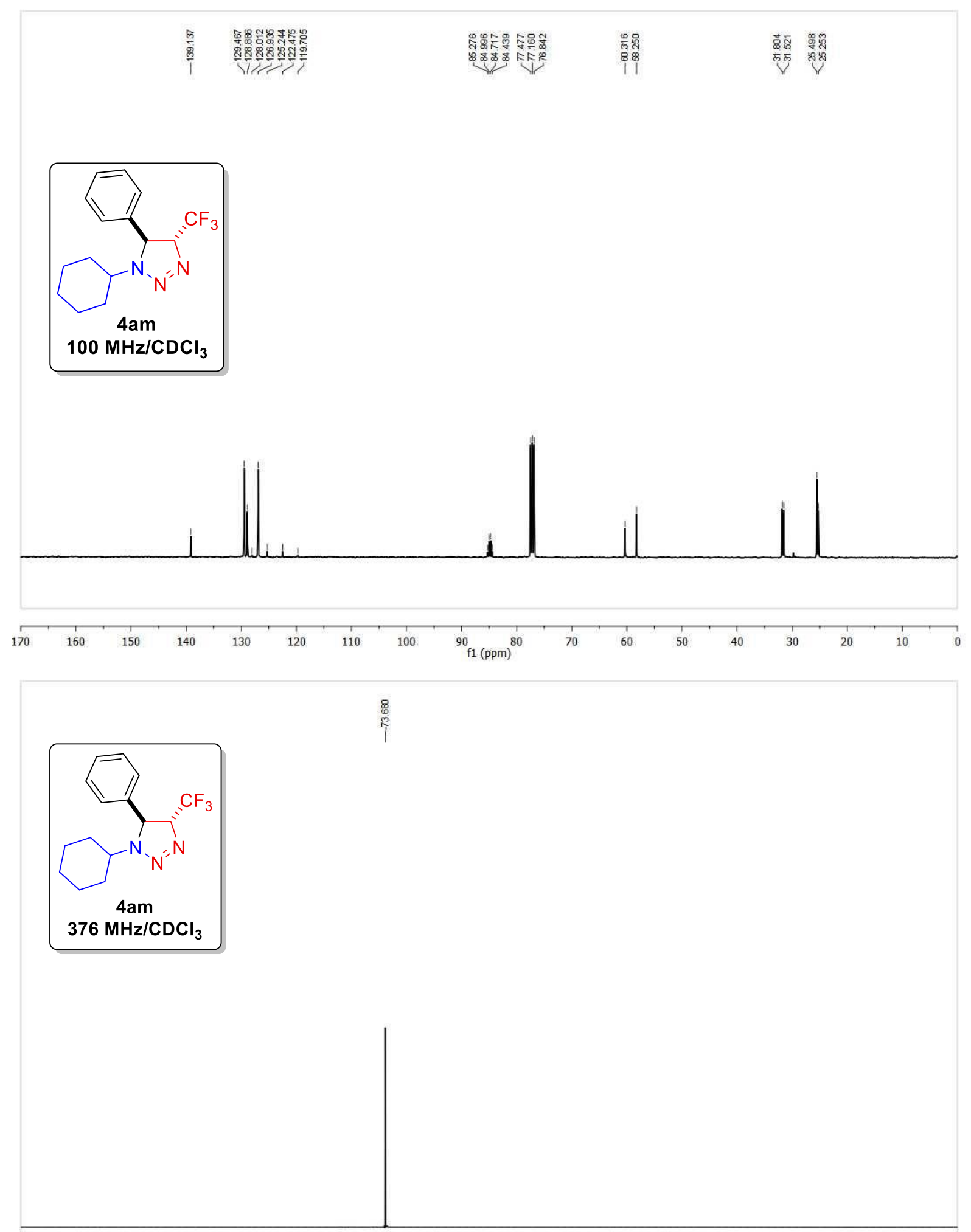

$\begin{array}{rllllllllllllllllllllllll}1 & 10 & 0 & -10 & -20 & -30 & -40 & -50 & -60 & -70 & -80 & -90 & -100 & -110 & -120 & -130 & -140 & -150 & -160 & -170 & -180 & -190 & -200 & -210 & \end{array}$ 

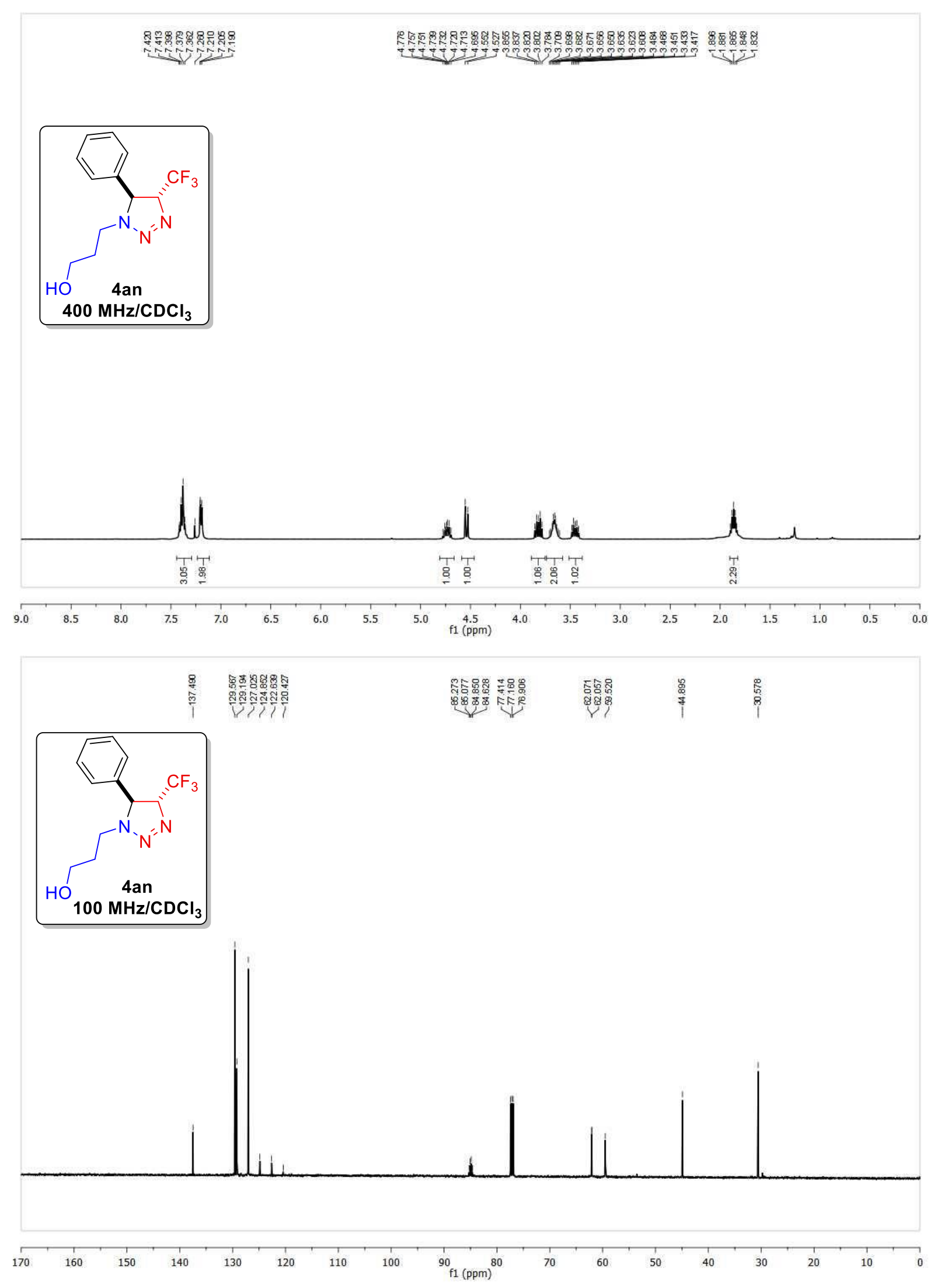


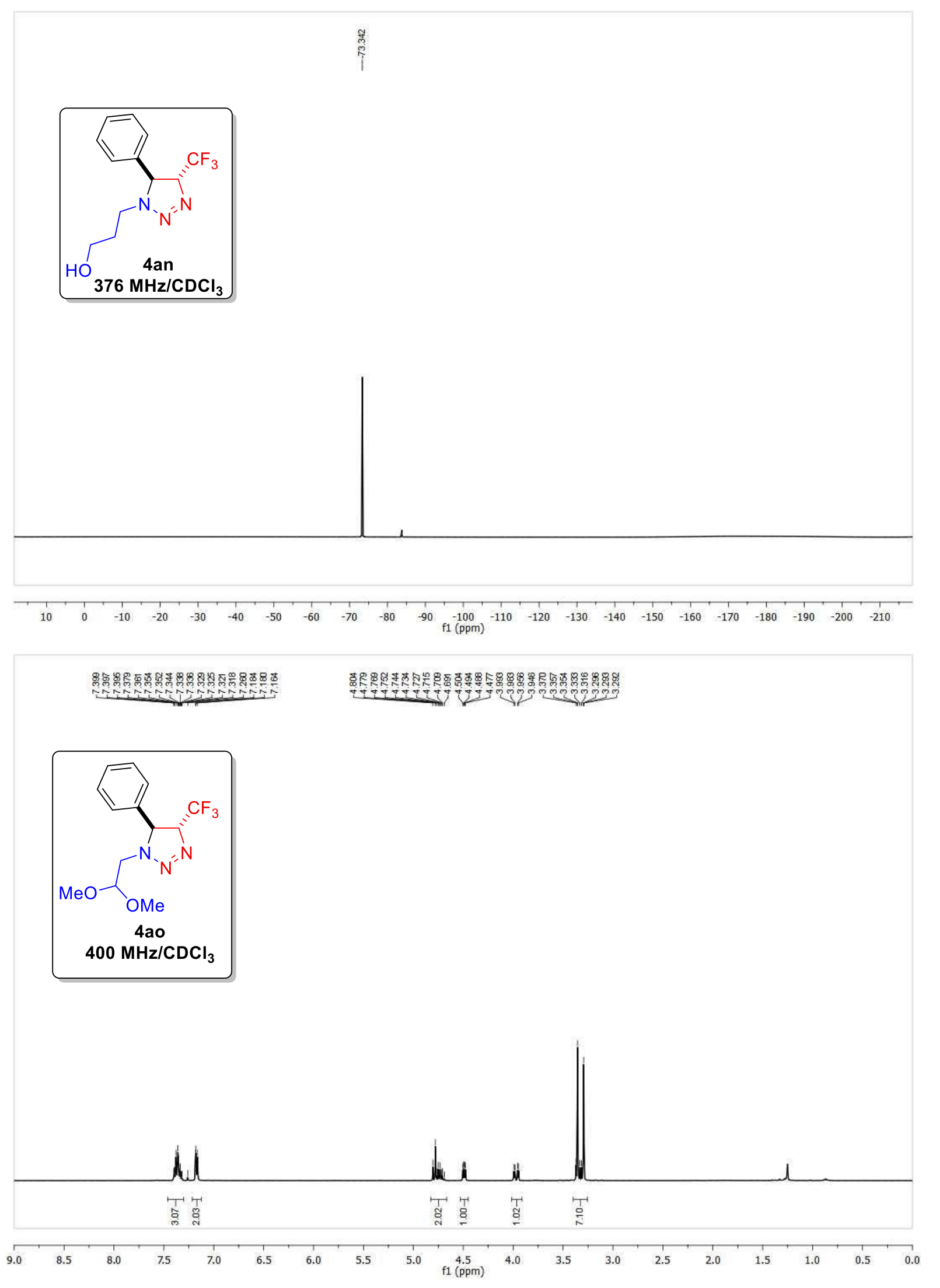



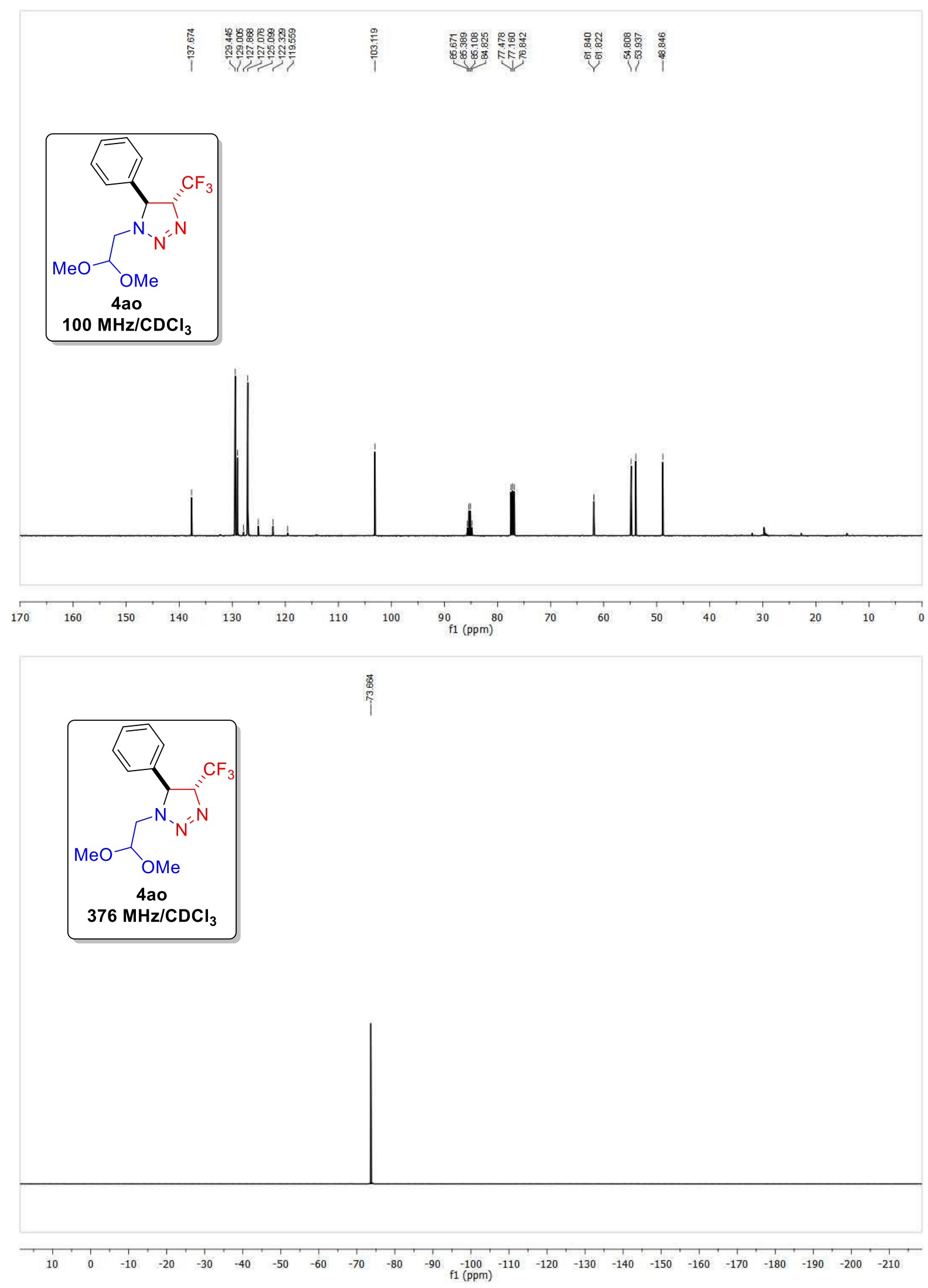

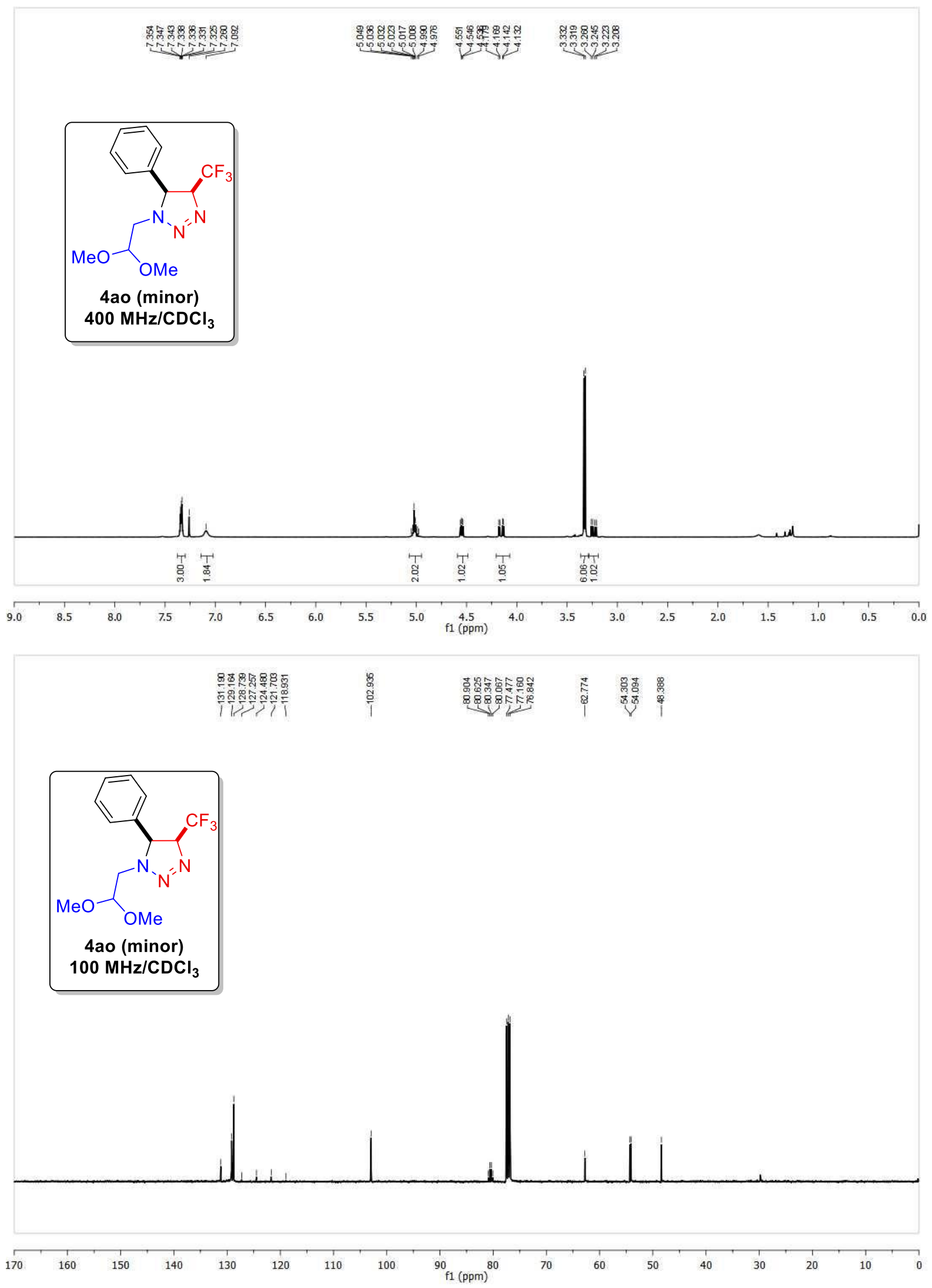


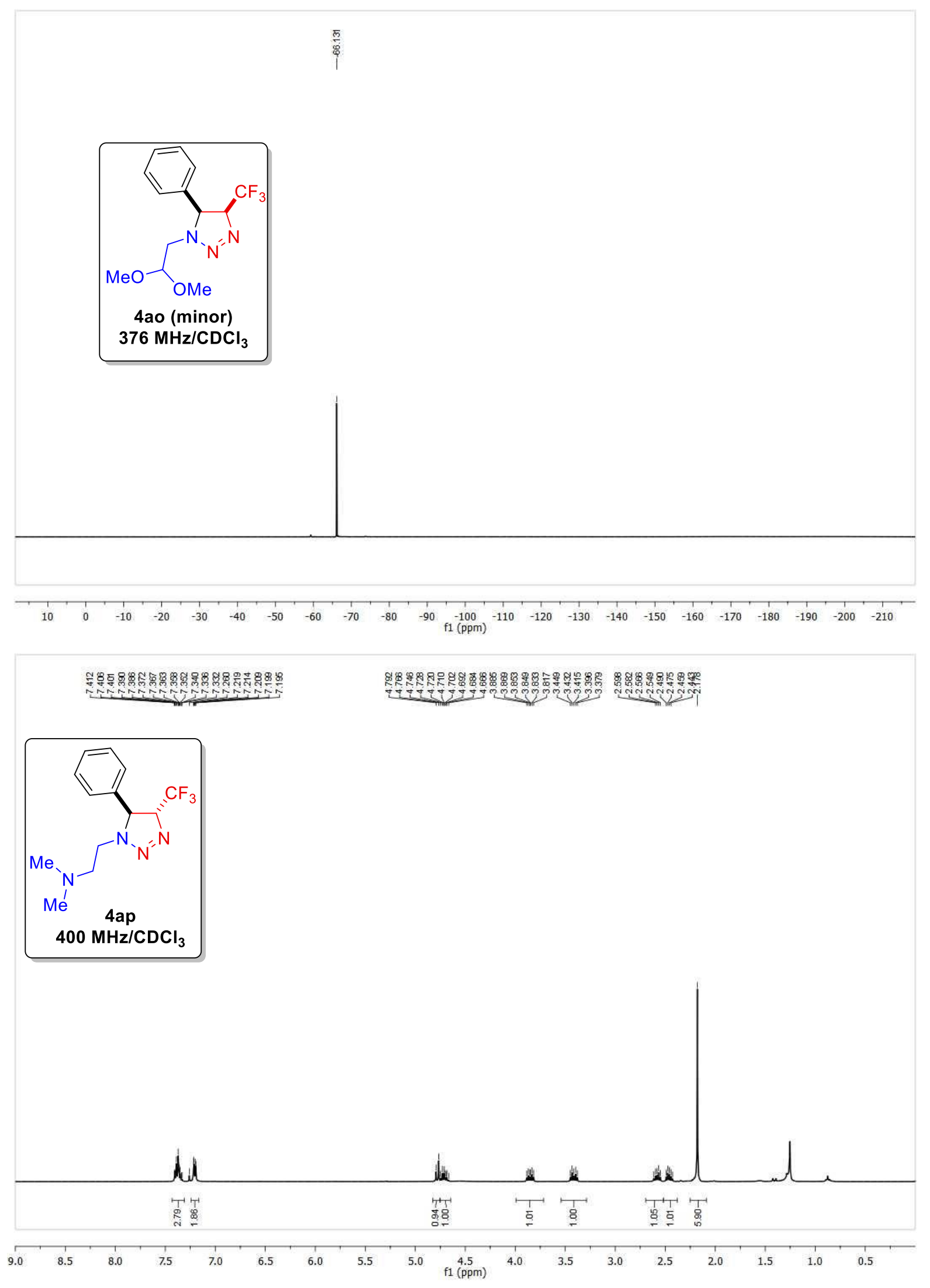



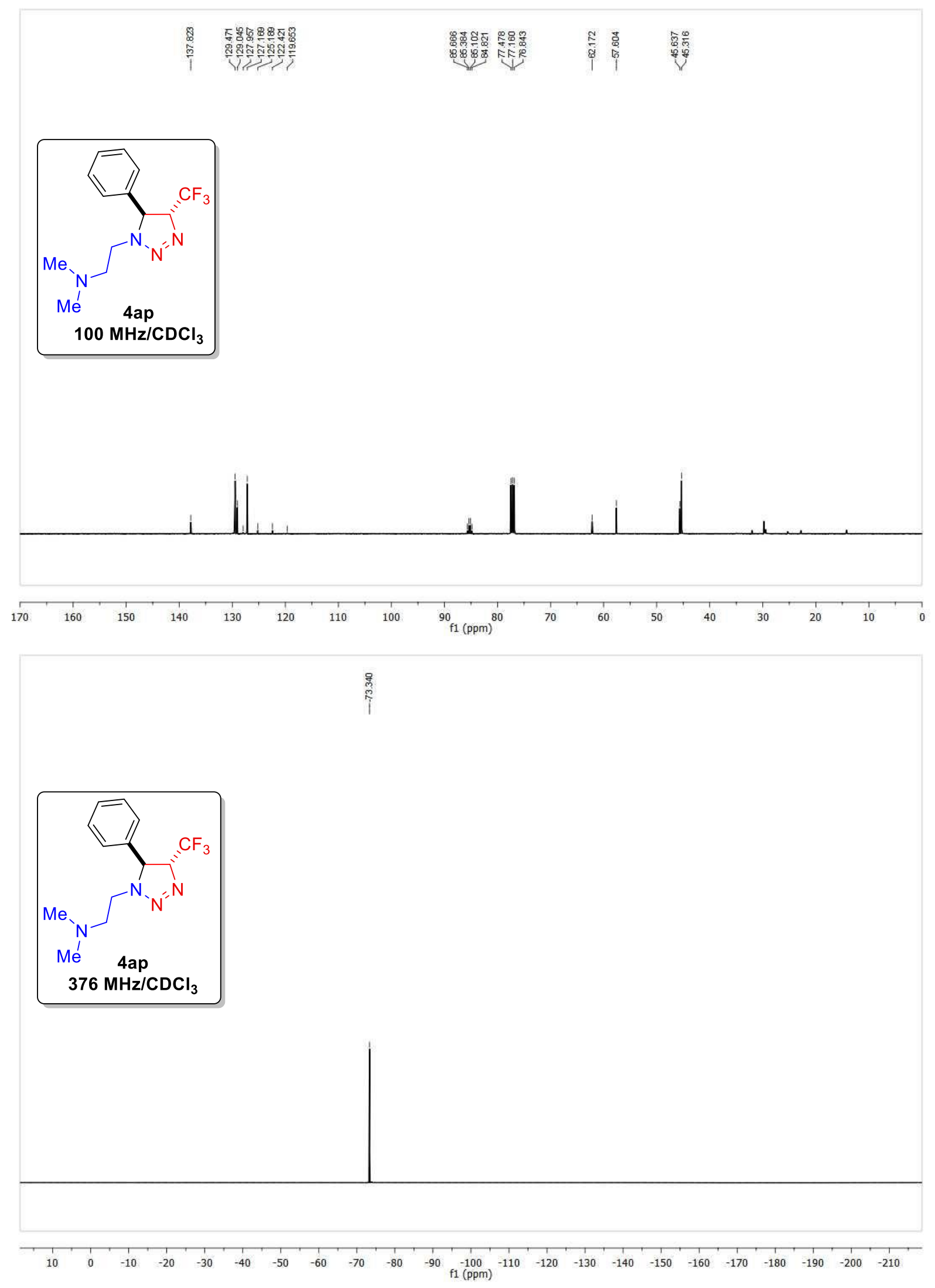

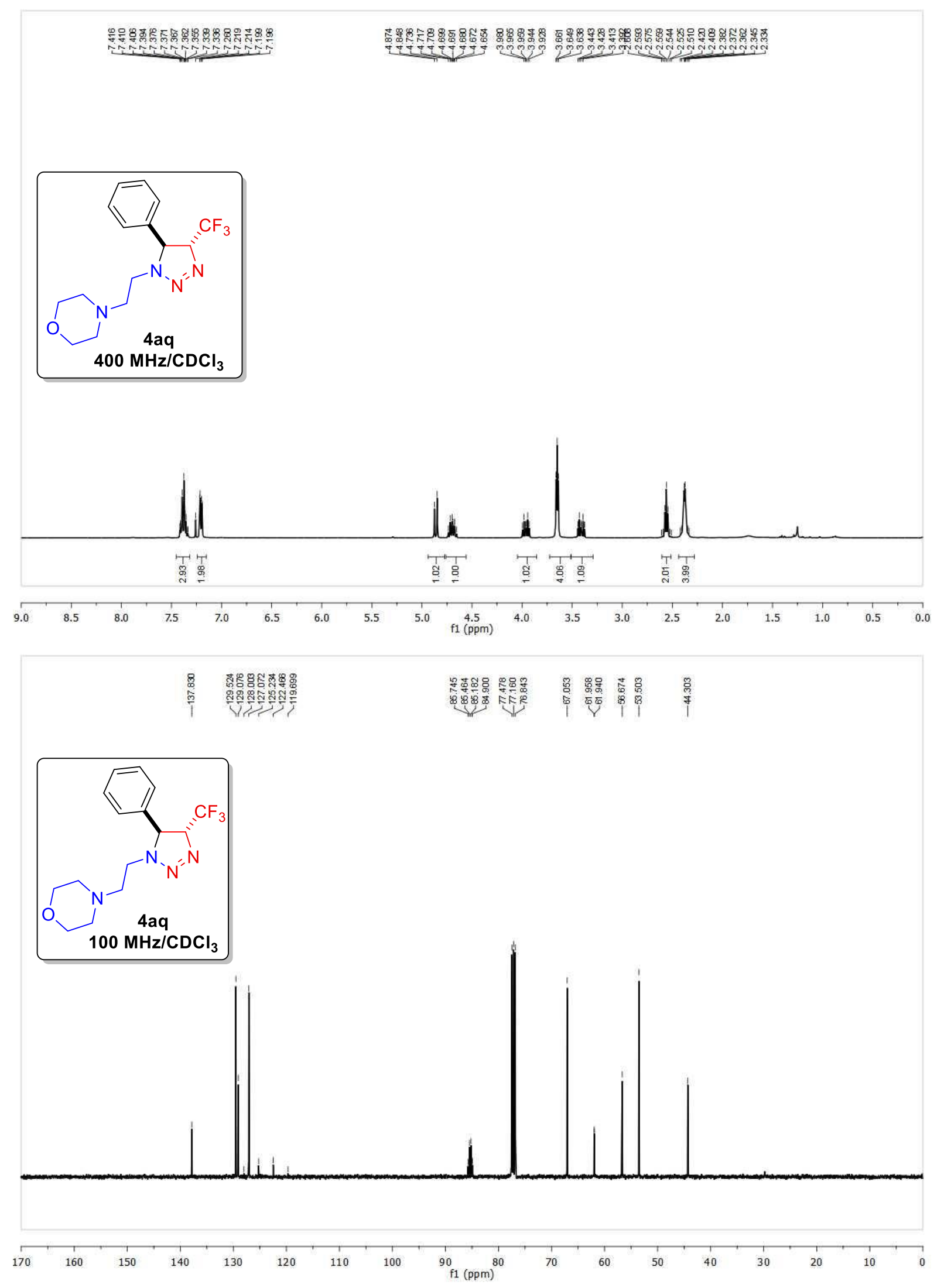


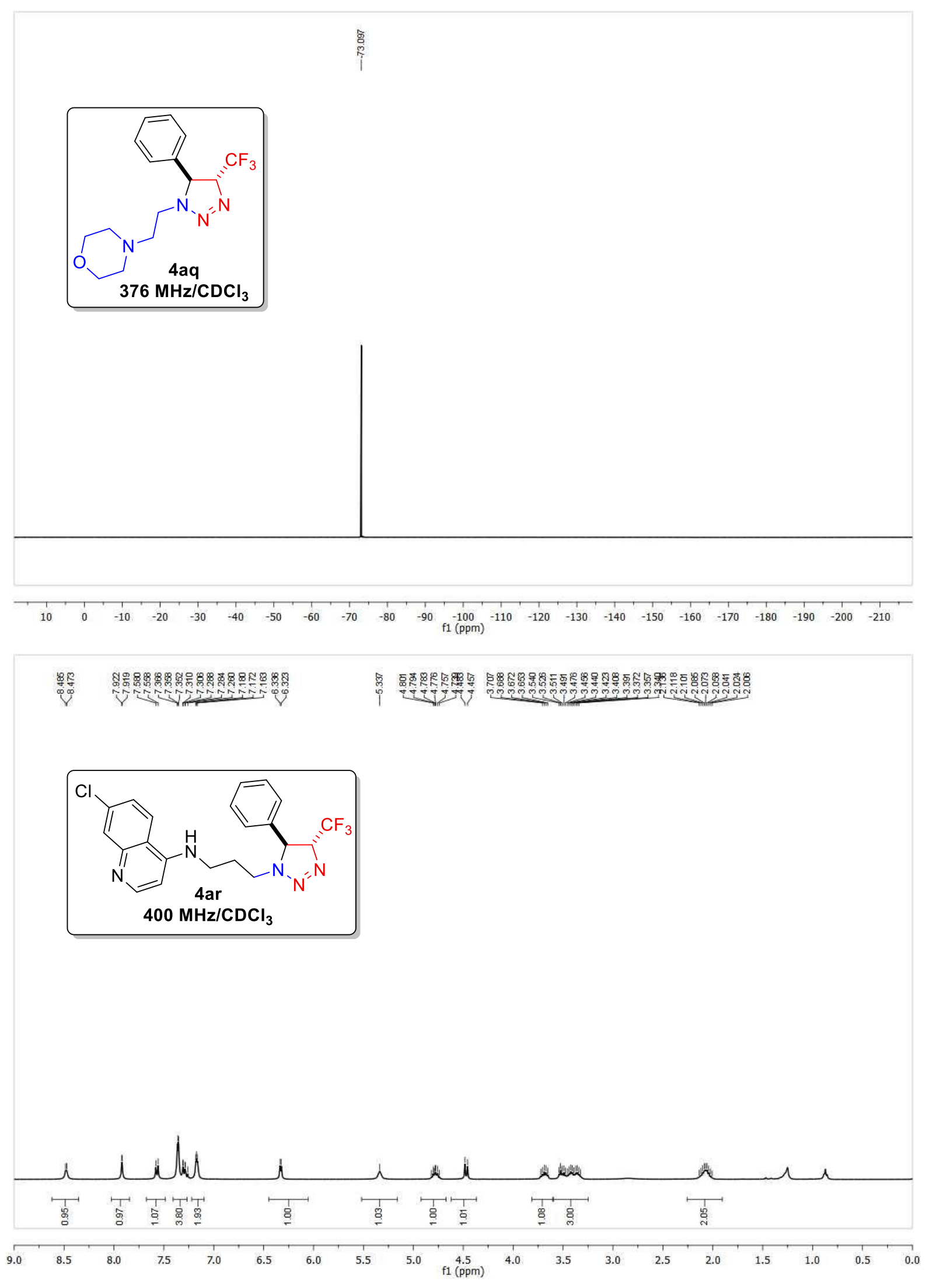



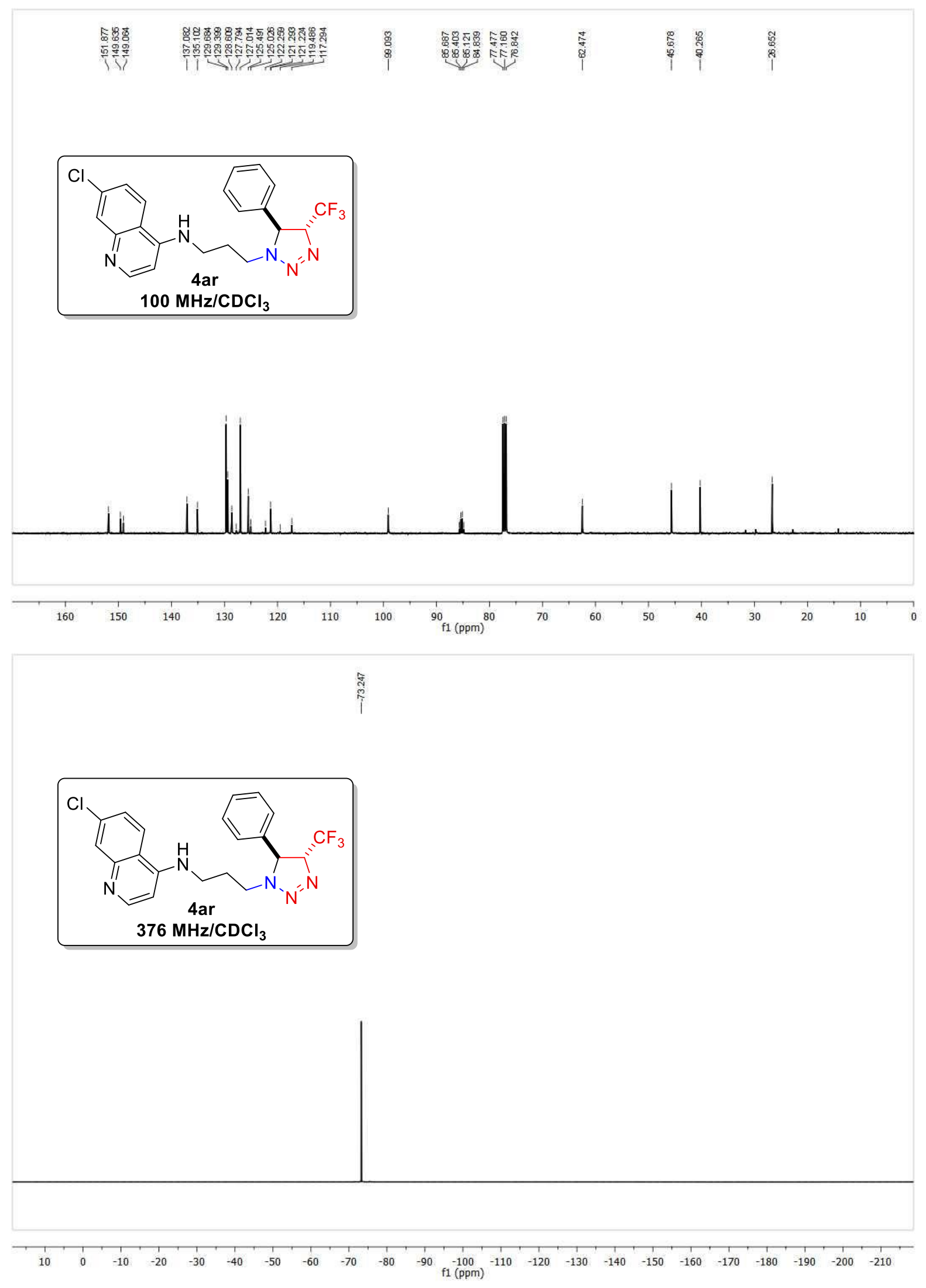

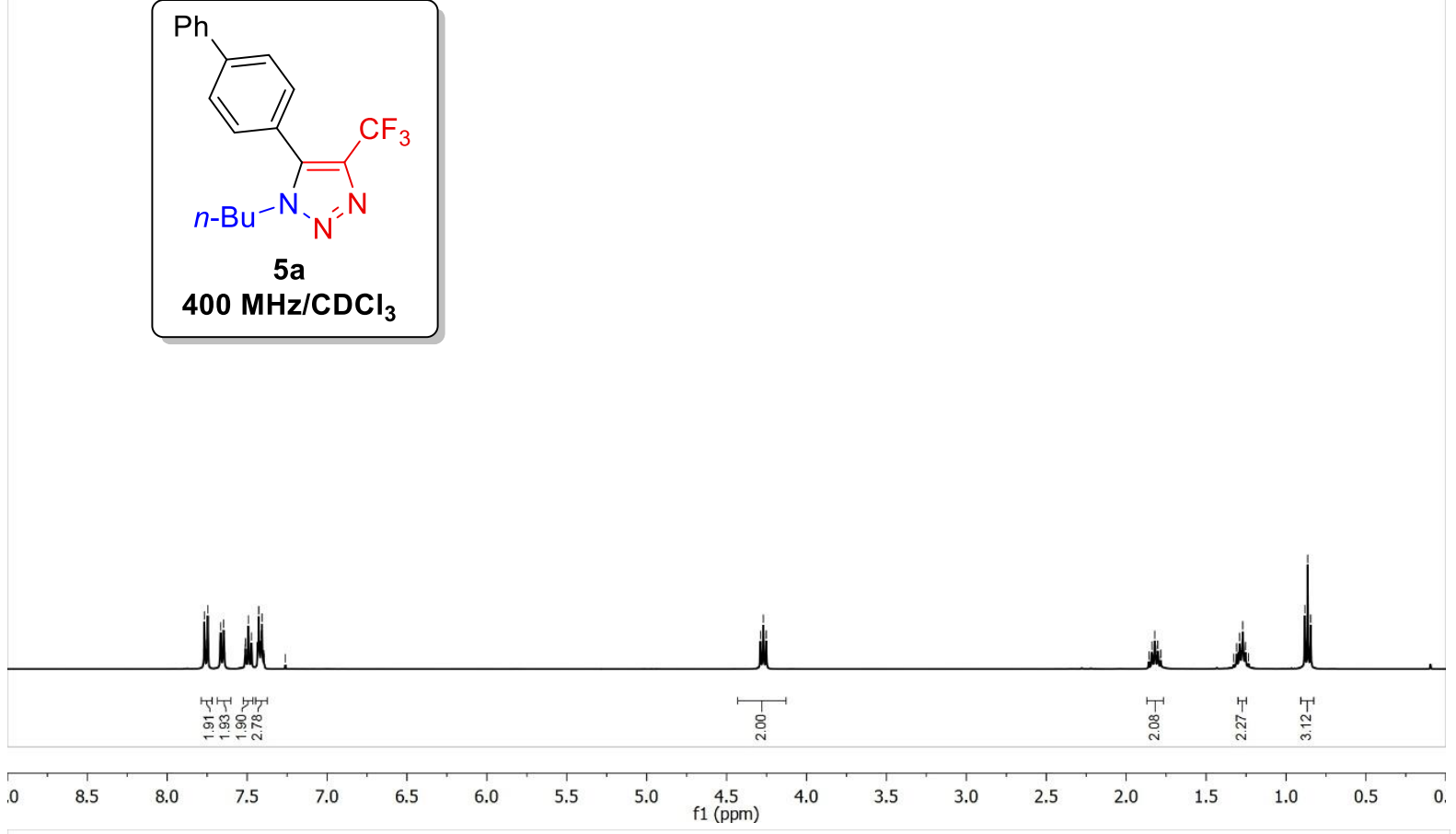

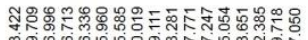

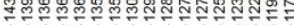

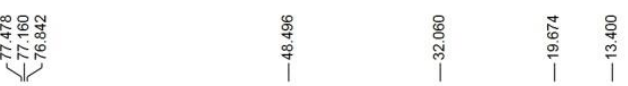
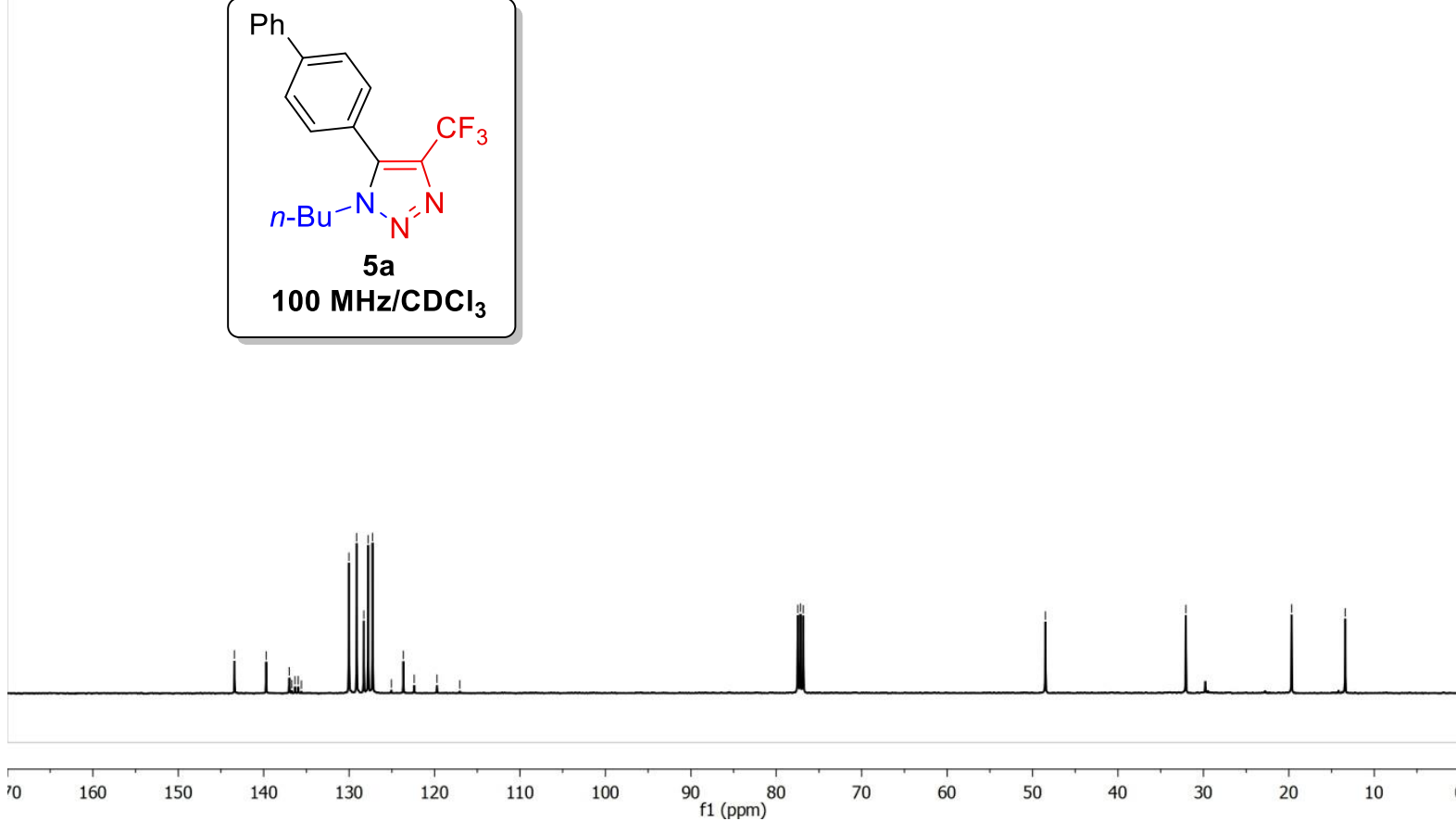


$$
376 \mathrm{MHz} / \mathrm{CDCl}_{3}
$$

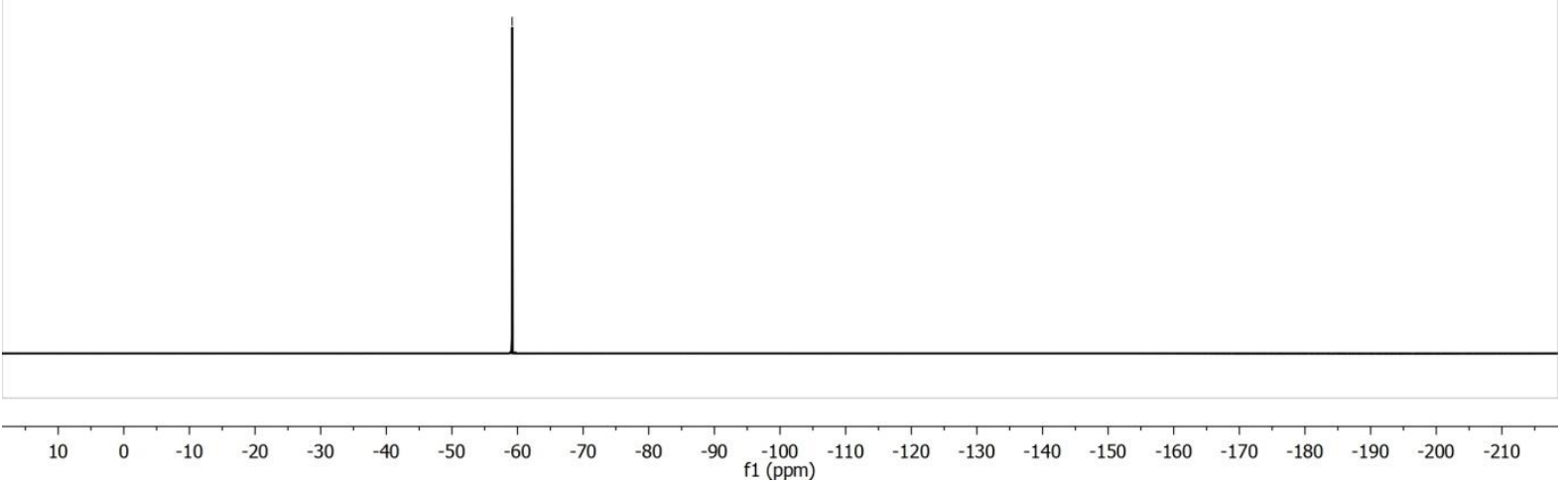

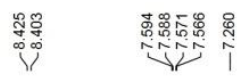

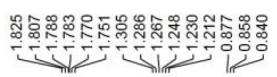
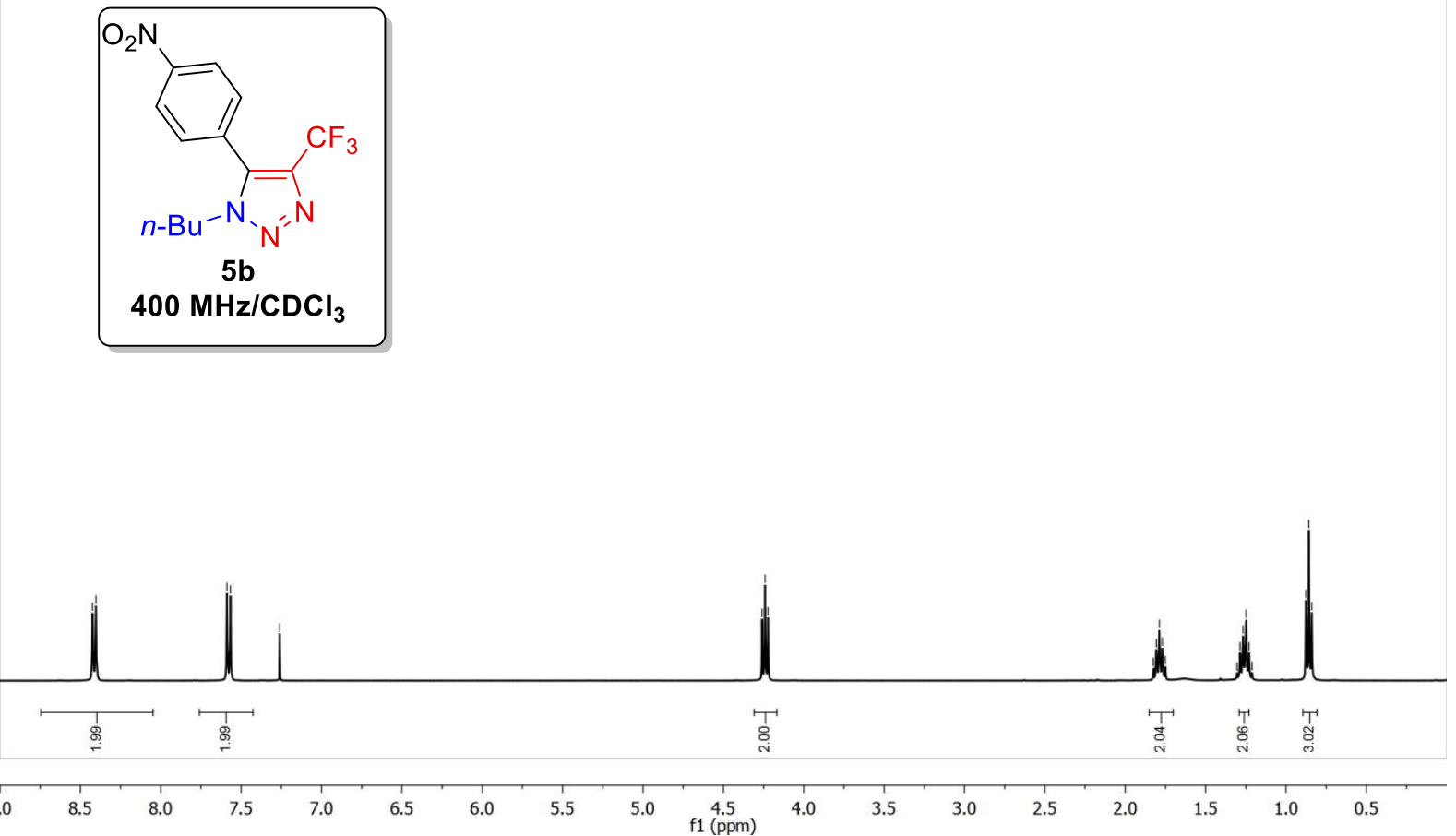


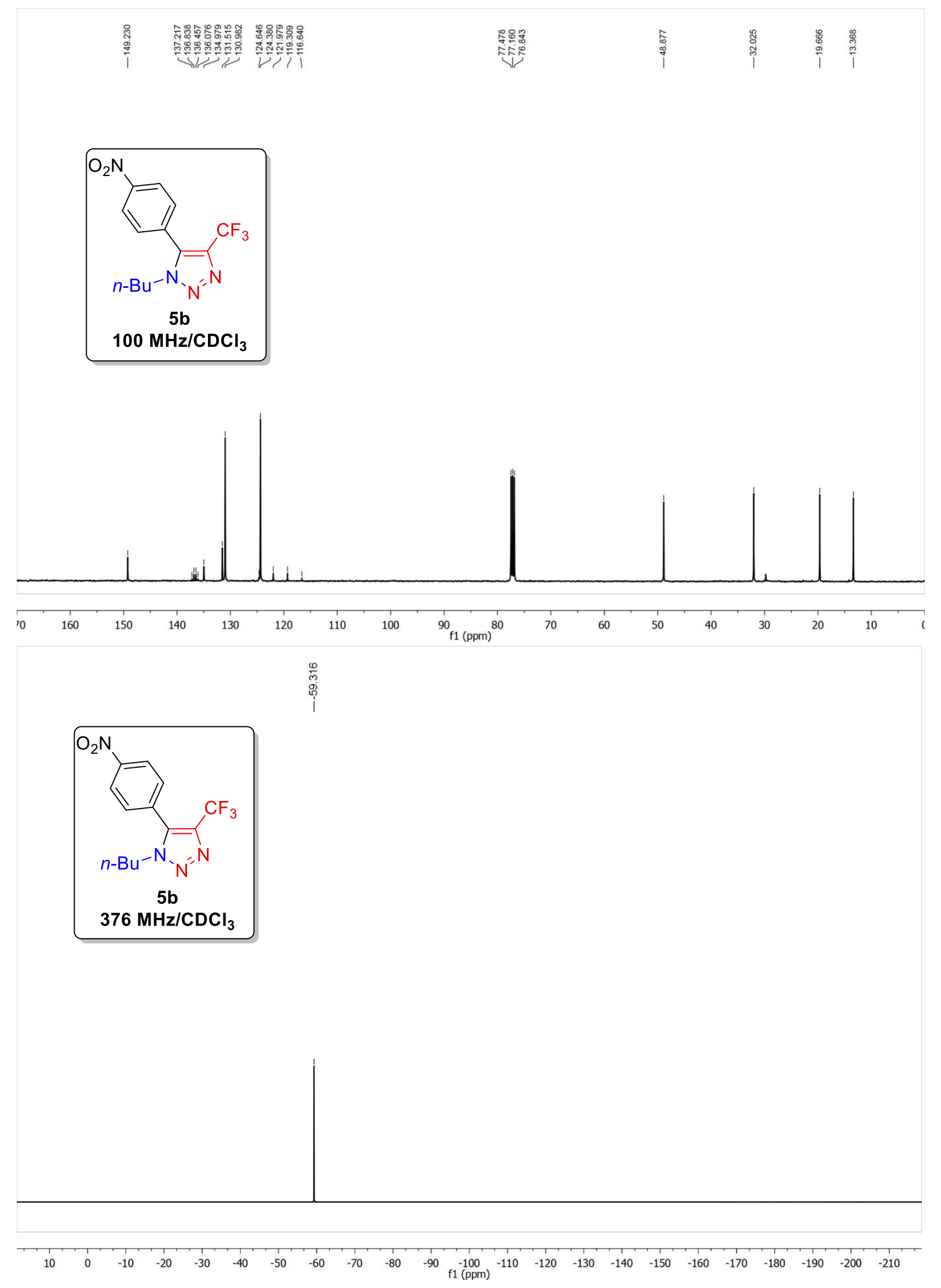




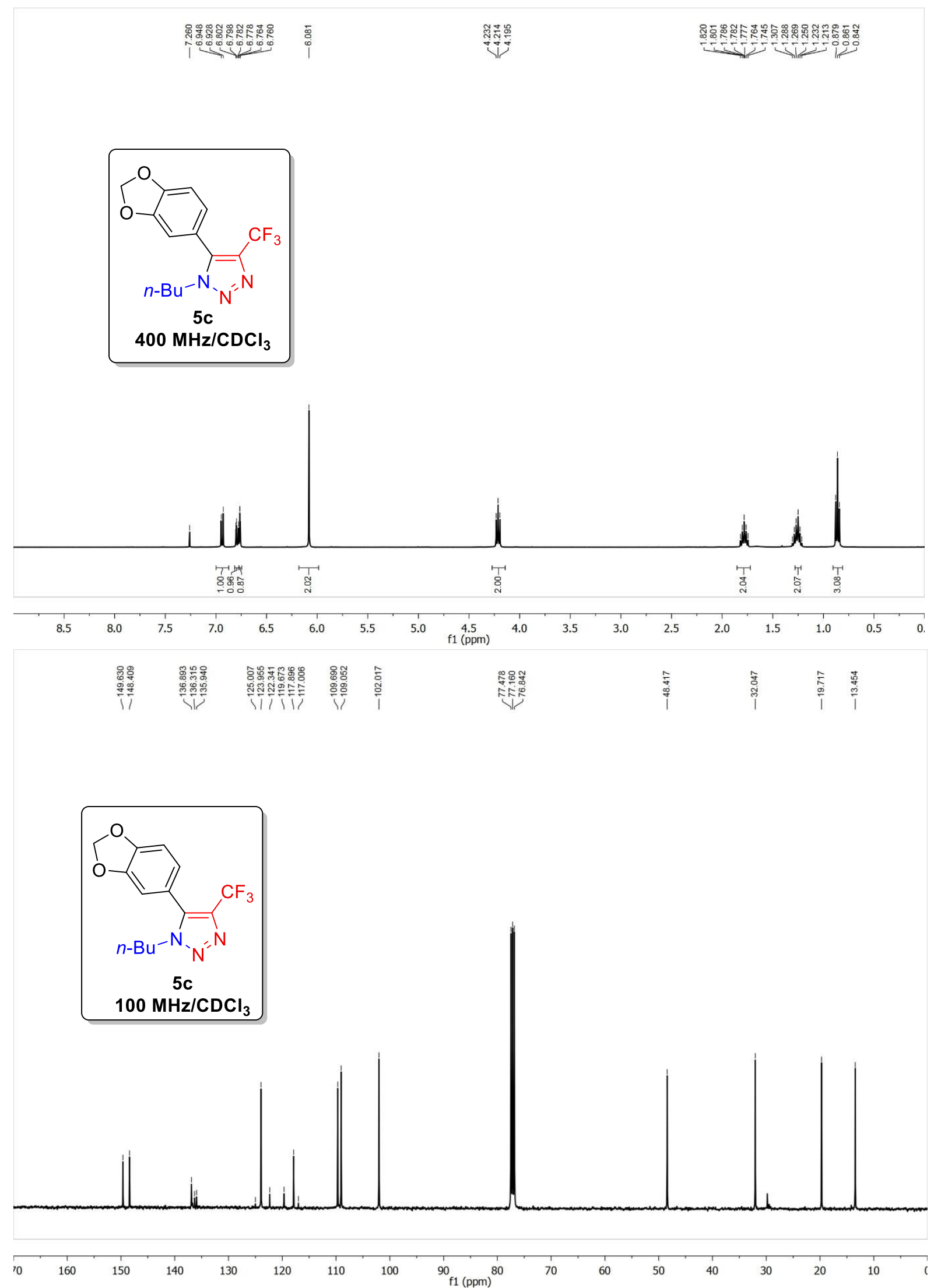




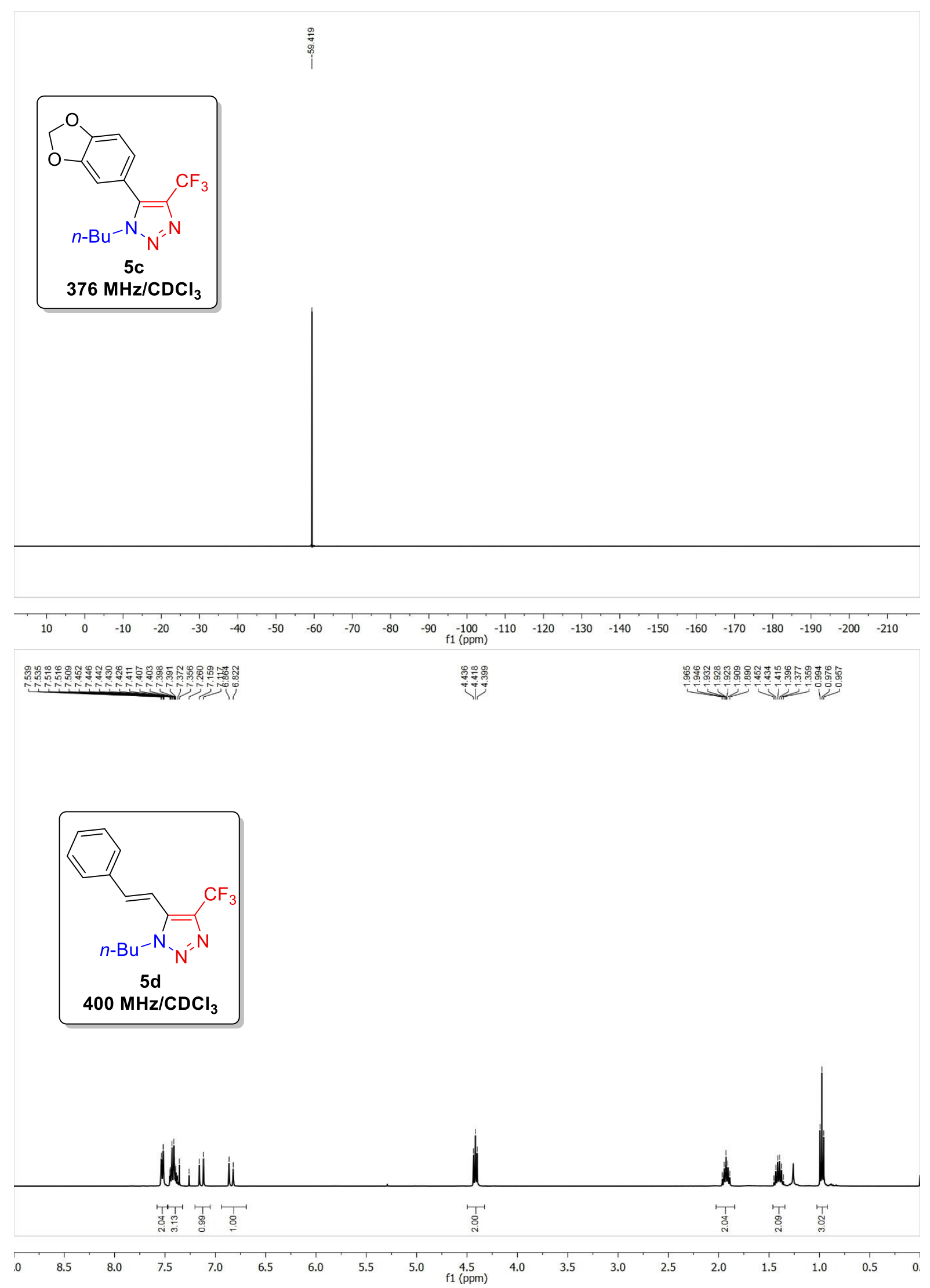



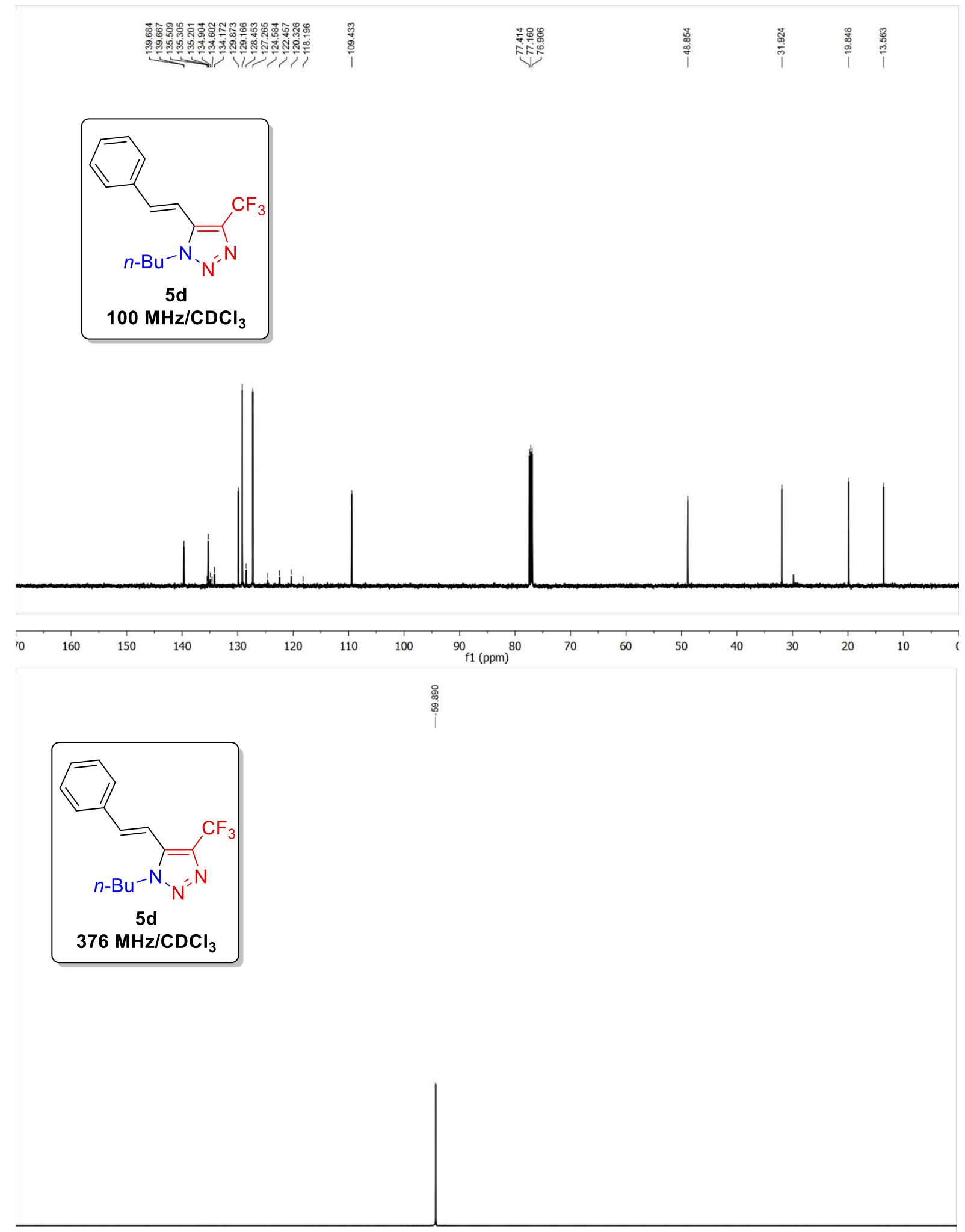

$\begin{array}{lllllllllllllllllllllllllllll}-40 & -42 & -44 & -46 & -48 & -50 & -52 & -54 & -56 & -58 & -60 & -62 & -64 & -66 & -68 & -70 & -72 & -74 & -76 & -78 & -80 & -82 & -84 & -86\end{array}$ 


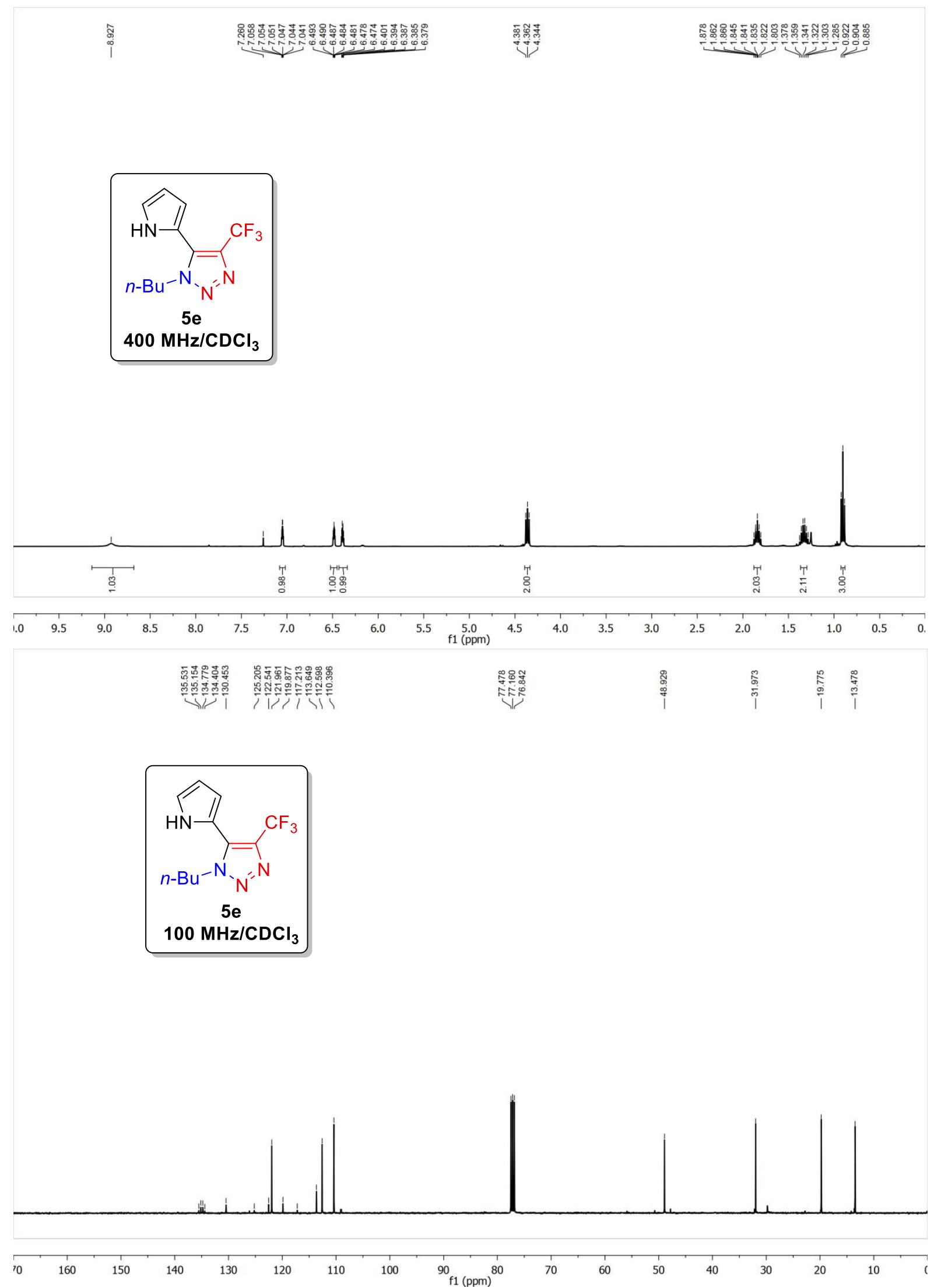




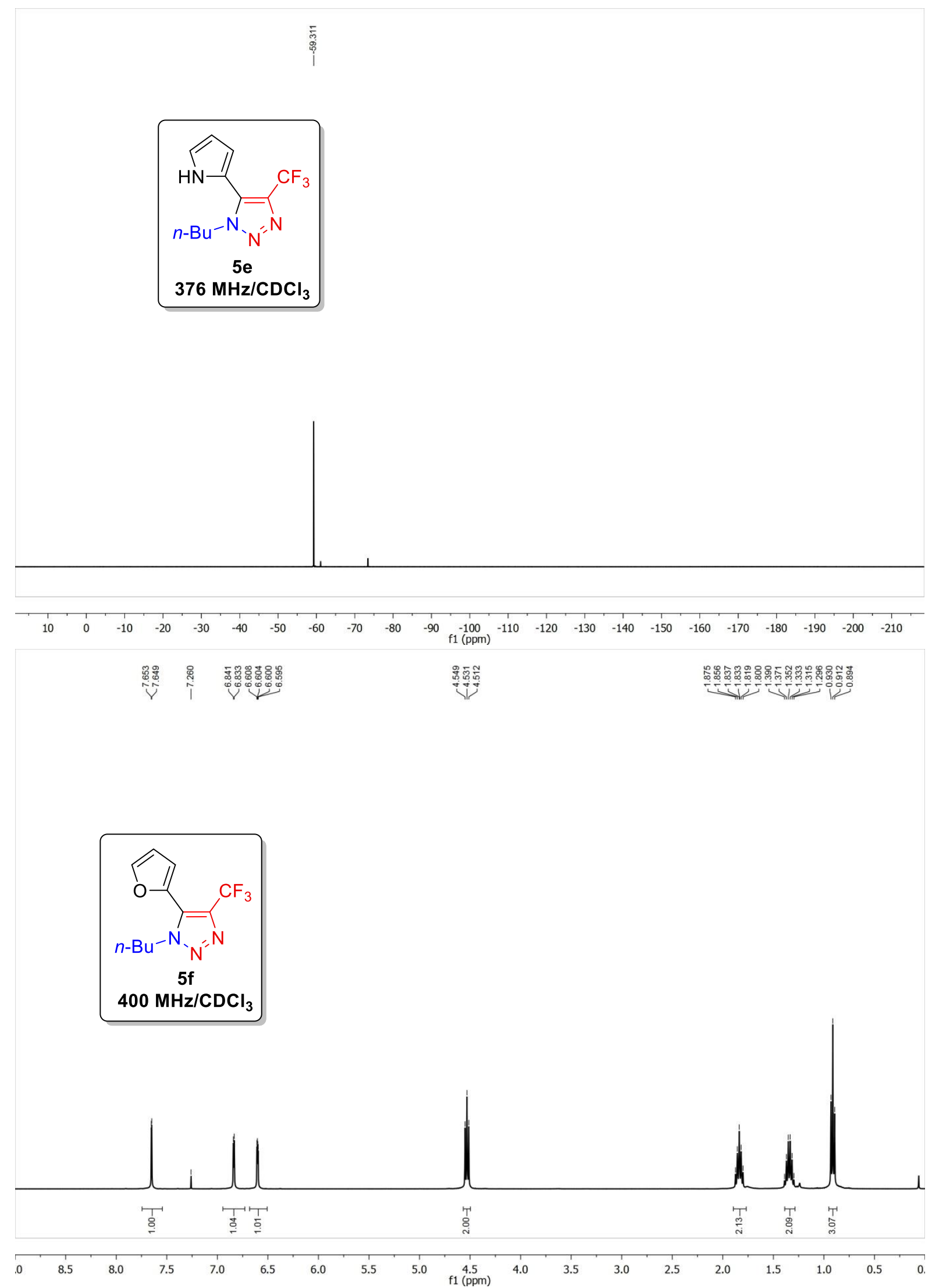




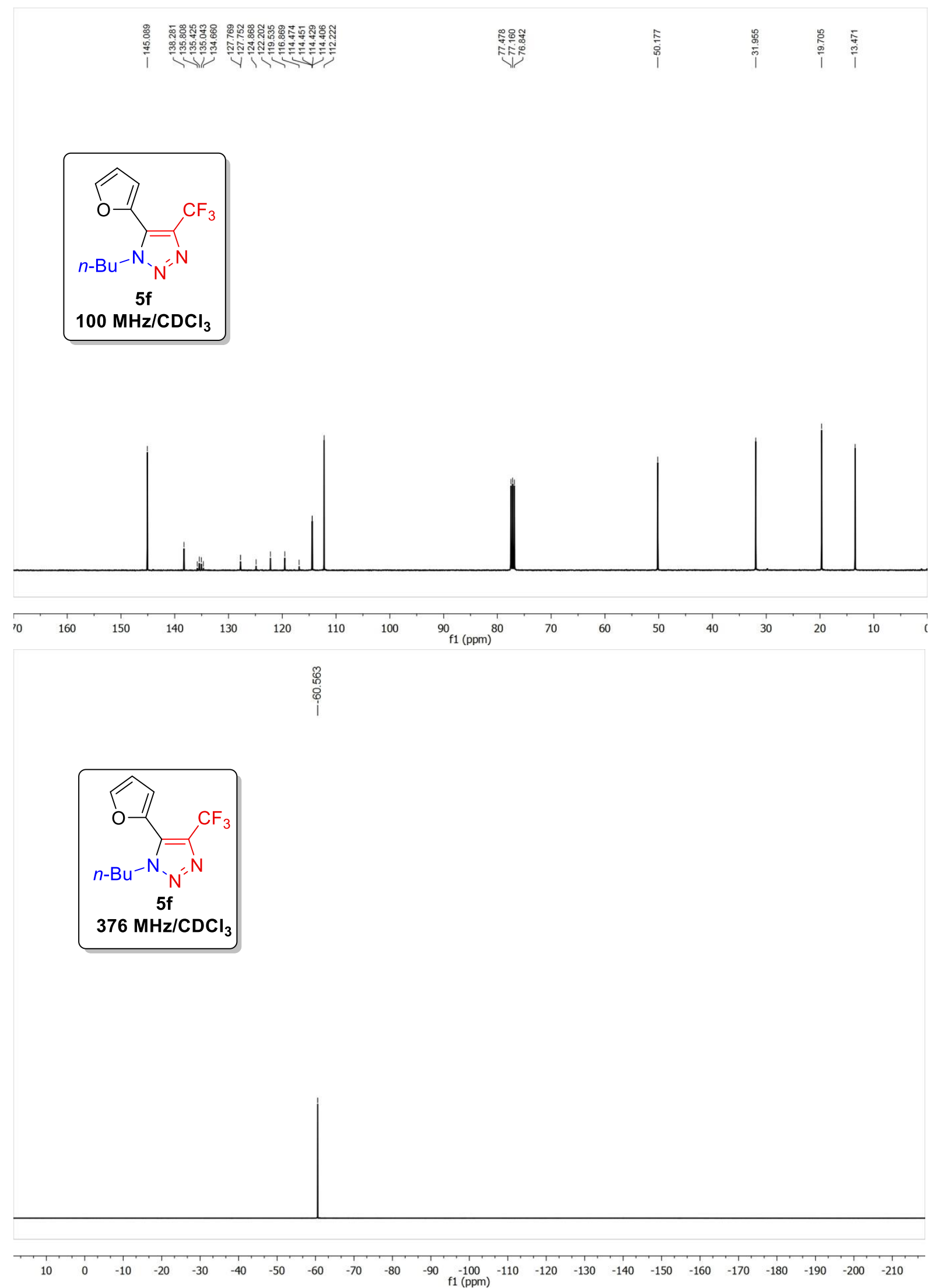




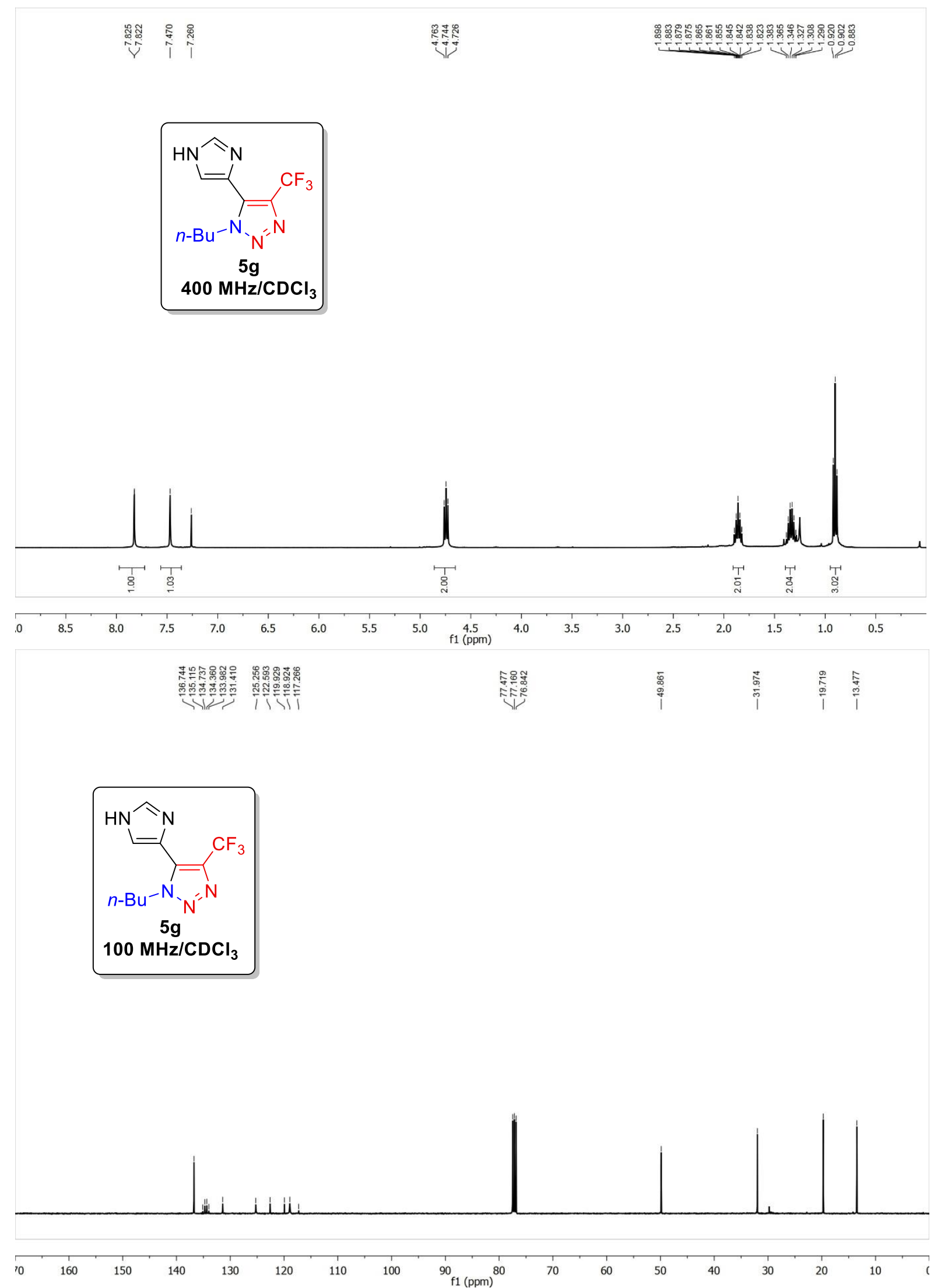




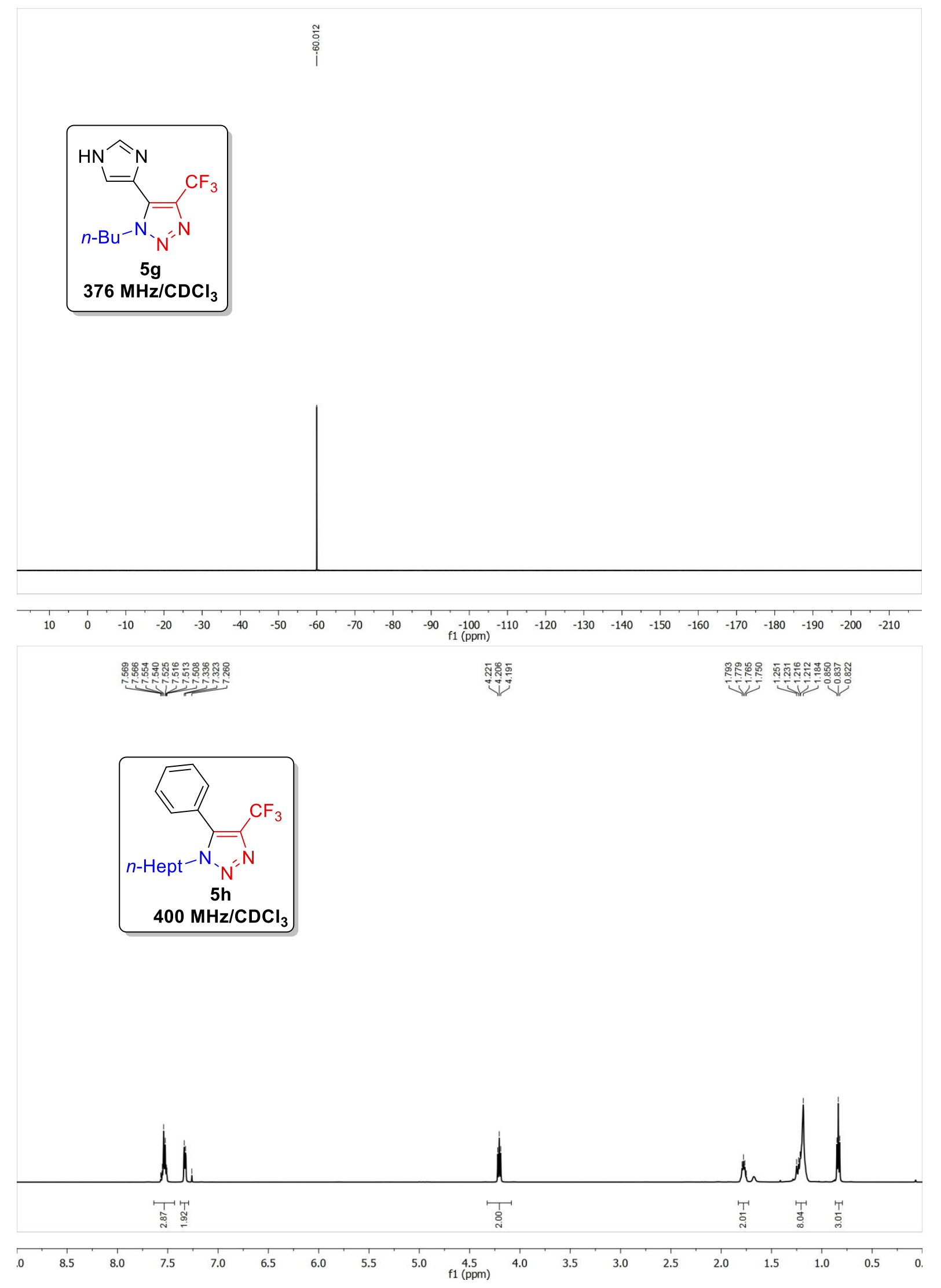




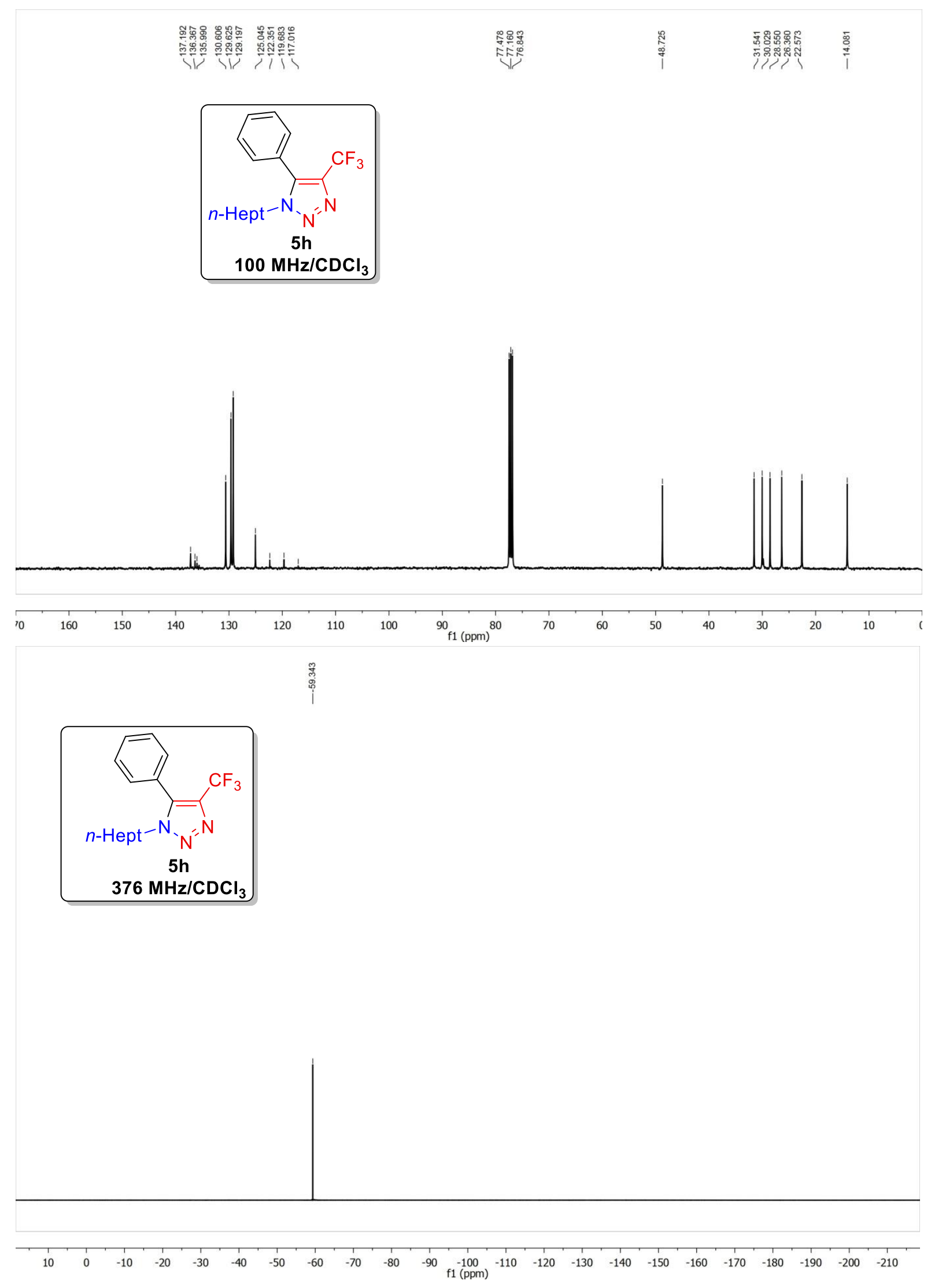




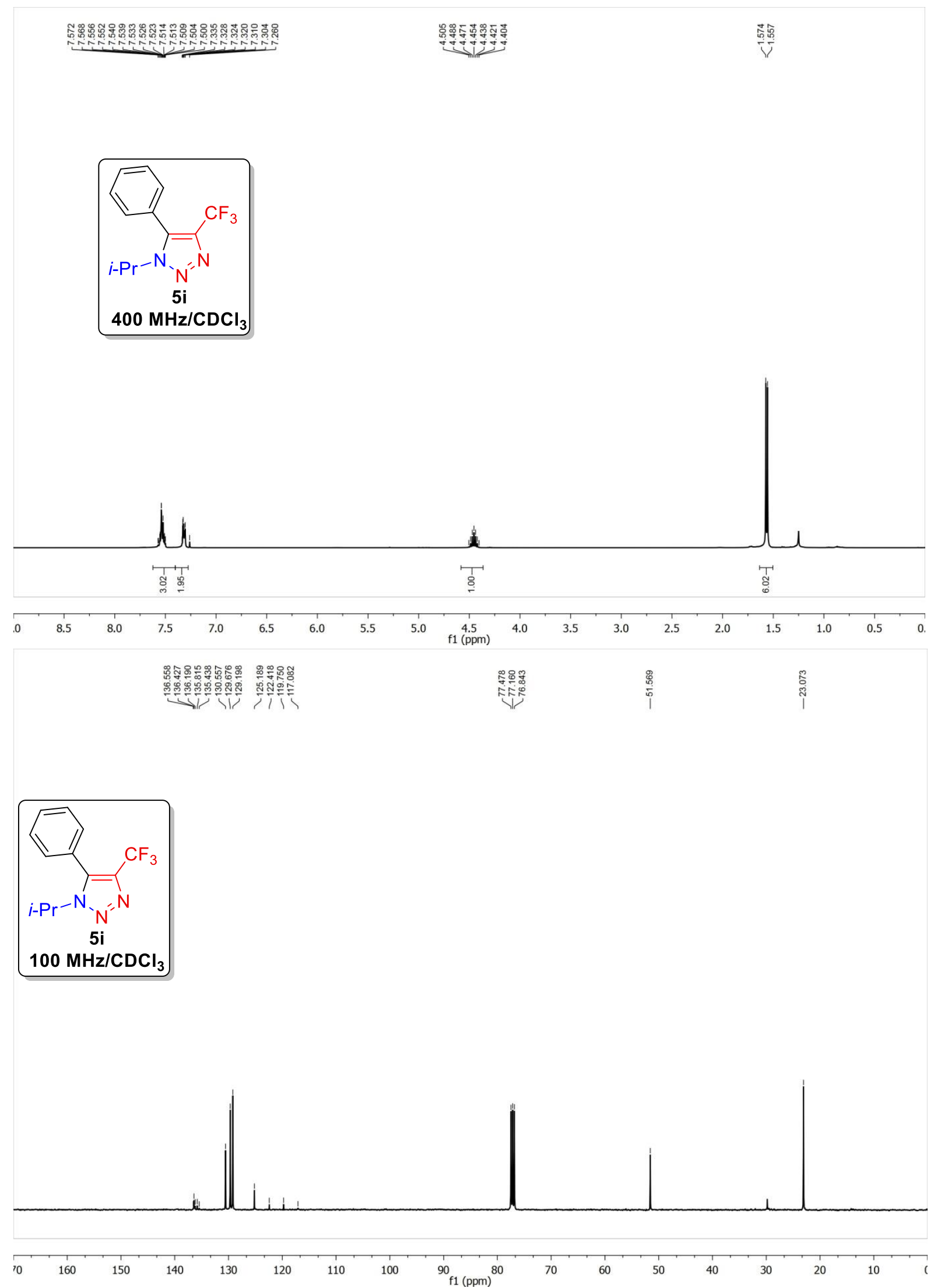




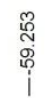

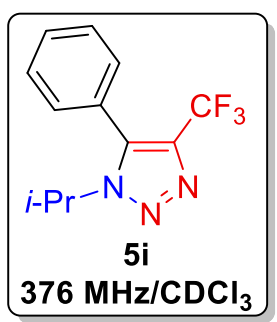

$\mid$\begin{tabular}{l|l}
$\mid$ \\
\end{tabular}

$\begin{array}{lllllllllllllllllllllll}10 & 0 & -10 & -20 & -30 & -40 & -50 & -60 & -70 & -80 & -90 & -100 & -110 & -120 & -130 & -140 & -150 & -160 & -170 & -180 & -190 & -200 & -210\end{array}$ 